\title{
Innovative Water Management Technology to Reduce Environmental Impacts of Produced Water
}

\author{
DE-NT0005682
}

FINAL REPORT

October 1, 2008 - May 15, 2013

James W. Castle ${ }^{1}$, Professor, Principal Investigator John H. Rodgers, Jr. ${ }^{2}$, Professor, Co-Principal Investigator Bethany Alley ${ }^{2}$, Graduate Research Assistant Alex Beebe ${ }^{1}$, Graduate Research Assistant Ruthanne Coffey ${ }^{1}$, Graduate Research Assistant Kristen Jurinko ${ }^{1}$, Graduate Research Assistant Michael Pardue ${ }^{1}$, Graduate Research Assistant Tina Ritter ${ }^{1}$, Graduate Research Assistant Michael M. Spacil ${ }^{2}$, Graduate Research Assistant

${ }^{1}$ Department of Environmental Engineering \& Earth Sciences ${ }^{2}$ School of Agricultural, Forest, and Environmental Sciences

Clemson University, Clemson, South Carolina

August 8, 2013

Submitted by:

Clemson University

300 Brackett Hall

Clemson, South Carolina 29634 
Acknowledgment: "This material is based upon work supported by the Department of Energy under Award Number DE-NT0005682."

Disclaimer: "This report was prepared as an account of work sponsored by an agency of the United States Government. Neither the United States Government nor any agency thereof, nor any of their employees, makes any warranty, express or implied, or assumes any legal liability or responsibility for the accuracy, completeness, or usefulness of any information, apparatus, product, or process disclosed, or represents that its use would not infringe privately owned rights. Reference herein to any specific commercial product, process, or service by trade name, trademark, manufacturer, or otherwise does not necessarily constitute or imply its endorsement, recommendation, or favoring by the United States Government or any agency thereof. The views and opinions of authors expressed herein do not necessarily state or reflect those of the United States Government or any agency thereof." 


\begin{abstract}
Clemson University with Chevron as an industry partner developed and applied treatment technology using constructed wetland systems to decrease targeted constituents in simulated and actual produced waters to achieve reuse criteria and discharge limits. Pilot-scale and demonstration constructed wetland treatment system (CWTS) experiments led to design strategies for treating a variety of constituents of concern (COCs) in produced waters including divalent metals, metalloids, oil and grease, and ammonia. Targeted biogeochemical pathways for treatment of COCs in pilot-scale CWTS experiments included divalent metal sulfide precipitation through dissimilatory sulfate reduction, metal precipitation through oxidation, reduction of selenite to insoluble elemental selenium, aerobic biodegradation of oil, nitrification of ammonia to nitrate, denitrification of nitrate to nitrogen gas, separation of oil using an oilwater separator, and sorption of ammonia to zeolite. Treatment performance results indicated that CWTSs can be designed and built to promote specific environmental and geochemical conditions in order for targeted biogeochemical pathways to operate. The demonstration system successfully achieved consistent removal extents even while inflow concentrations of COCs in the produced water differed by orders of magnitude. Design strategies used in the pilot-scale and demonstration CWTSs to promote specific conditions that can be applied to designing full-scale CWTSs include plant and soil selection, water-depth selection, addition of amendments, and hydraulic retention time (HRT). These strategies allow conditions within a CWTS to be modified to achieve ranges necessary for the preferred biogeochemical treatment pathways. In the case of renovating a produced water containing COCs that require different biogeochemical pathways for treatment, a CWTS can be designed with sequential cells that promote different conditions. For example, the pilot-scale CWTS for post-reverse osmosis produced water was designed to promote oxidizing conditions within the first wetland cell for nitrification of ammonia, and the subsequent three cells were designed to promote reducing conditions for denitrification of nitrate. By incorporating multiple wetland cells in a CWTS, the conditions within each cell can be modified for removal of specific COCs. In addition, a CWTS designed with multiple cells allows for convenient sample collection points so that biogeochemical conditions of individual cells can be monitored and performance evaluated. Removal rate coefficients determined from the pilot-scale CWTS experiments and confirmed by the demonstration system can be used to calculate HRTs required to treat COCs in full-scale CWTSs. The calculated HRTs can then be used to determine the surface area or "footprint" of a full-size CWTS for a given inflow rate of produced water.
\end{abstract}




\section{TABLE OF CONTENTS}

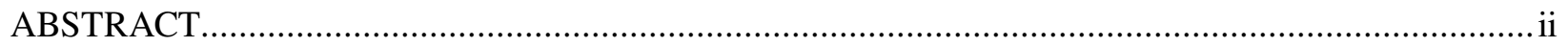

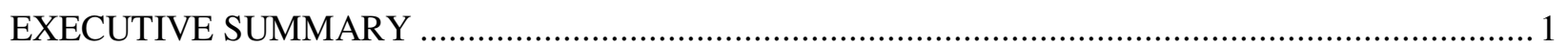

INTRODUCTION

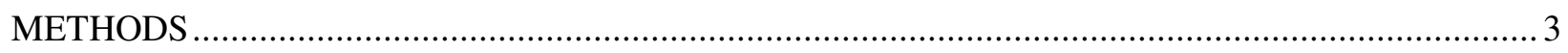

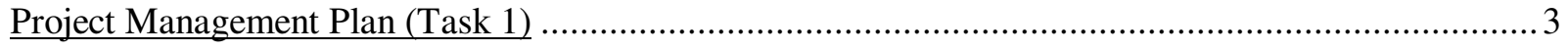

Identify Chemical, Physical, and Risk Characteristics of Produced Waters (Task 2) .......................... 3

Determine Reuse and Discharge Criteria and Develop Treatment Performance Goals (Task 3) ................ 3

Design and Construct Pilot-Scale Constructed Wetland Treatment Systems (Task 4) ........................ 4

Measure Treatment Performance of Pilot-Scale Constructed Wetland Systems (Task 5)..................... 4

Assess Pilot-Scale Performance (Task 6) ............................................................................... 4

Design and Construct Demonstration Constructed Wetland Treatment System (Task 7) ....................... 4

Measure Treatment Performance of Demonstration Constructed Wetland System (Task 8) ...................5

Apply Results to Water Management and Technology Replication (Task 9) …....................................5

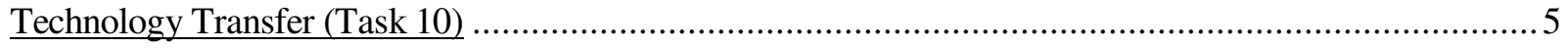

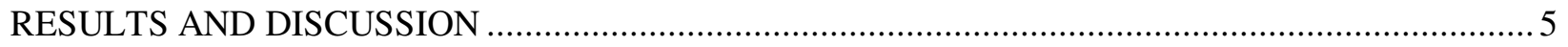

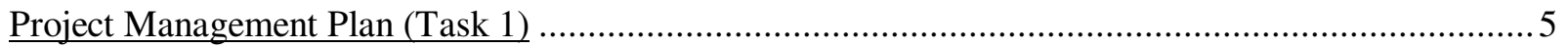

Identify Chemical, Physical, and Risk Characteristics of Produced Waters (Task 2) ..........................5

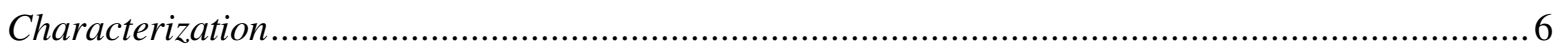

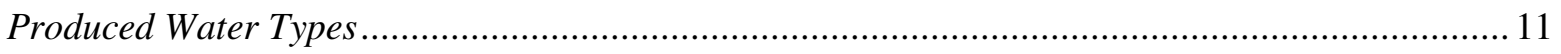

Determine Reuse and Discharge Criteria and Develop Treatment Performance Goals (Task 3) .............. 17

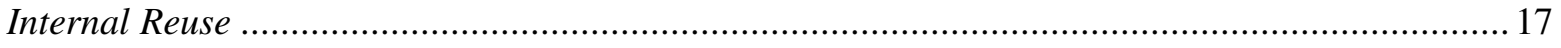

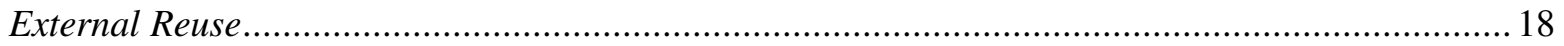

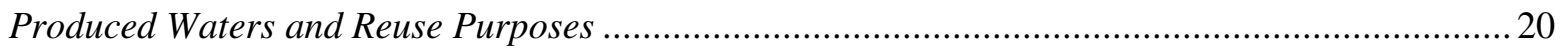

Design and Construct Pilot-Scale Constructed Wetland Treatment Systems (Task 4)............................ 30

Pilot-Scale CWTS for Simulated Post-RO Produced Water .......................................................... 31

Pilot-Scale CWTS for PW Containing Divalent Metals and Low Molecular Weight Organics......... 33

Pilot-Scale CWTS for PW Containing Metalloids and Low Molecular Weight Organics................. 34

Pilot-Scale CWTS for PW Containing Oil \& Grease and Divalent Metals .................................... 35

Measure Treatment Performance of Pilot-Scale Constructed Wetland Systems (Task 5).................... 40

Pilot-Scale CWTS for Simulated Post-RO Produced Water ....................................................... 41

Pilot-Scale CWTS for PW Containing Divalent Metals and Low Molecular Weight Organics......... 45 
Pilot-Scale CWTS for PW Containing Metalloids and Low Molecular Weight Organics.....

Pilot-Scale CWTS for PW Containing Oil \& Grease and Divalent Metals

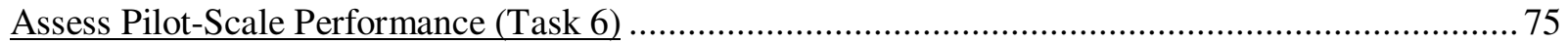

Pilot-Scale CWTS for Simulated Post-RO Produced Water ........................................................ 75

Pilot-Scale CWTS for PW Containing Divalent Metals and Low Molecular Weight Organics.........76

Pilot-Scale CWTS for PW Containing Metalloids and Low Molecular Weight Organics................. 77

Pilot-Scale CWTS for PW Containing Oil \& Grease and Divalent Metals .................................... 77

Design and Construct Demonstration Constructed Wetland Treatment System (Task 7) ....................... 83

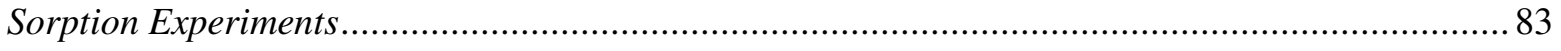

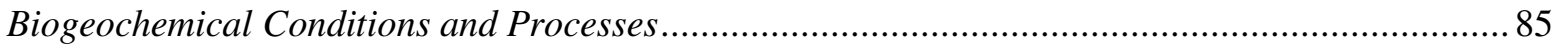

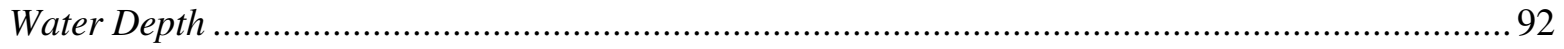

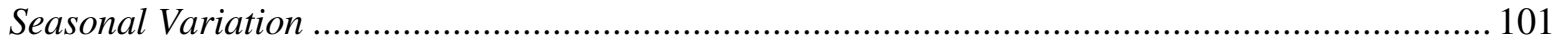

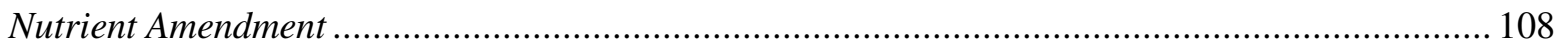

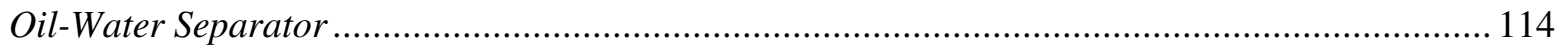

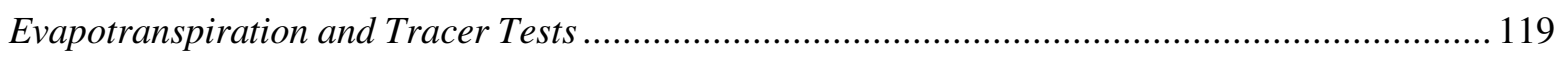

Design and Construction of Demonstration Wetland Treatment System ..................................... 123

Measure Treatment Performance of Demonstration Constructed Wetland System (Task 8) ................ 130

Apply Results to Water Management and Technology Replication (Task 9).................................. 160

Application to Various Geologic Basins and Locations ........................................................... 160

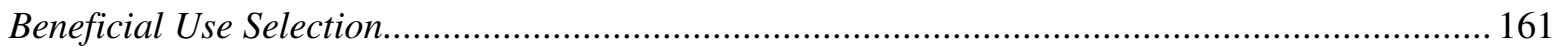

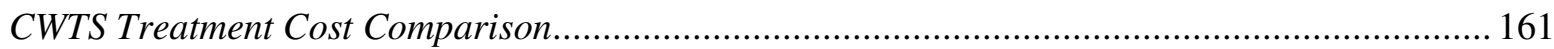

Produced Water Management Plan and Application to Treatment System Design ...................... 163

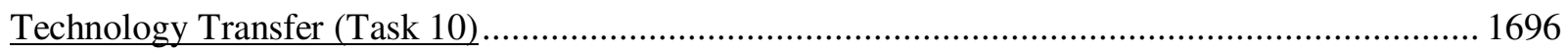

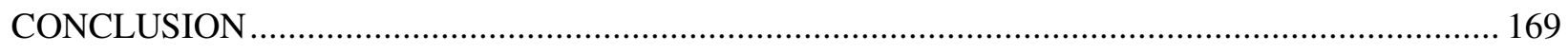

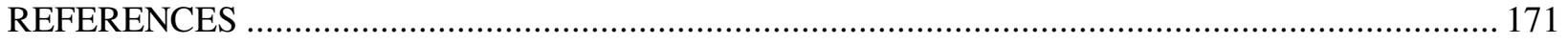




\section{EXECUTIVE SUMMARY}

Clemson University with Chevron as an industry partner developed and applied technology to decrease targeted constituents in simulated and actual produced waters to achieve reuse criteria and discharge limits. Phase I of the project included assessment of environmental factors associated with produced waters that impact development of U.S. oil and gas reserves, particularly factors pertinent to application of constructed wetland systems for the treatment of produced waters. Phase I began with completion of a Project Management Plan (Task 1). Chemical, physical, and risk characteristics of produced waters were identified in Task 2. A literature search was completed, and characterization data for specific types of produced waters were compiled. The data indicate a wide range of characteristics, with produced waters containing various metals, organics (e.g. oil and grease), and other constituents. Reuse and discharge criteria and specific treatment performance goals were determined in Task 3 . The results of Tasks 2 and 3, including identification of constituents of concern in produced waters and development of treatment performance goals, were important for moving ahead to Phase II and designing systems to provide treatment for water discharge or reuse.

Phase II involved investigation of prototype (pilot-scale) constructed wetland treatment systems. The pilot-scale systems were designed and constructed in Task 4. Treatment performance of the pilot-scale systems was measured (Task 5) and assessed (Task 6). Performance measurements from the pilot-scale constructed wetland systems demonstrated successful design strategies for treating a variety of constituents of concern including divalent metals, metalloids, oil and grease, and ammonia. Targeted pathways for treating these constituents in the pilot-scale experiments included divalent metal sulfide precipitation through dissimilatory sulfate reduction, metal precipitation through oxidation, reduction of selenite to insoluble elemental selenium, aerobic biodegradation of oil, nitrification of ammonia to nitrate, denitrification of nitrate to nitrogen gas, separation of oil using an oil-water separator, and sorption of ammonia to zeolite.

Experiments in support of designing demonstration and full-scale constructed wetland treatment systems were performed to gain a better understanding of conditions affecting treatment pathways and hybridized design strategies that can be incorporated into constructed wetland treatment. Experiments included comparing seedling growth of corn, cabbage, and soybean between untreated and treated simulated produced water. The difference in early seedling growth between the waters was determined to be statistically significant, with higher growth rate in the treated water. Toxicity tests using Ceriodaphnia dubia and Pimephales promelas compared the potential between untreated and treated simulated produced water to cause adverse effects to receiving system biota. Statistical analysis indicated a significant difference in the responses of $C$. dubia and $P$. promelas between the untreated and treated water, with greater survival in the treated water. Evapotranspiration data and comparison of theoretical lysimeter outflow determined using the Penman Monteith equation with measured lysimeter outflow demonstrated application of the equation to estimating evapotranspiration in the pilot-scale constructed wetland treatment systems used in this investigation. Additional experiments were performed to determine the effect of plant transpiration on transport of constituents through the hydrosoil and the effects of seasonal changes on wetland performance. Wetland cells containing Typha latifolia were more effective at transporting constituents through the hydrosoil than wetland cells that did not contain Typha as indicated by shorter tracer breakthrough times. 
The influence of water depth on treatment performance of the pilot-scale constructed wetlands for renovating simulated fresh oilfield produced water was investigated. Divalent metal concentrations were greater in outflow from wetland cells having 15 and $23 \mathrm{~cm}$ water depths than from cells having 46 and $56 \mathrm{~cm}$ water depths. The cells having 46 and $56 \mathrm{~cm}$ water depths provided reducing conditions conducive for dissimilatory sulfate reduction, which contributed to removal of divalent metals from the simulated produced water. Wetland cells with 15 and $23 \mathrm{~cm}$ water depths were more effective for removing oil in the simulated produced water than cells with greater water depths because the shallower depth promoted conditions favorable for aerobic degradation of oil.

Phase III included on-site treatment of produced water using a demonstration constructed wetland system. In collaboration with Chevron, a site for constructing the demonstration system was selected near Berry, Alabama. This site was within a producing coalbed methane field. Data from the pilot-scale experiments were used in Task 7 to design and construct the demonstration system. The system consisted of four wetland series designed to treat the following constituents of concern in water produced from the field: ammonia, barium, cadmium, iron, manganese, nitrate, and selenium. The system included sequentially arranged oxidizing and reducing cells designed to promote conditions identified during Phase II for targeted treatment pathways. Monitoring of treatment performance of the demonstration constructed wetland system (Task 8) from September 2012 through March 2013 indicated effective removal of constituents of concern, even as inflow concentrations of these constituents in the produced water varied. Sampling and analytical methods were used that allowed for calculation of removal efficiency, removal rate, and removal extent in order to assess treatment performance.

Results were applied to developing strategies for water management and technology replication in Task 9. Design strategies used in the pilot-scale and demonstration wetland treatment systems that can be applied to designing full-scale constructed wetland systems for treating waters ranging from fresh to saline include plant and soil selection, water-depth selection, addition of amendments, hydraulic retention time, and hybrid components. These strategies allow conditions within a wetland treatment system to be modified to achieve ranges necessary for the preferred biogeochemical treatment pathways. In the case of renovating a produced water containing constituents of concern that require different biogeochemical pathways for treatment, a constructed wetland system can be designed with sequential cells that promote different conditions. Removal rate coefficients determined from the pilot-scale constructed wetland experiments and confirmed by the demonstration wetland treatment system can be used to calculate hydraulic retention times required to treat constituents of concern in full-scale constructed wetland systems. The calculated hydraulic retention times can then be used to determine the surface area or "footprint" of a full-size constructed wetland treatment system for a given inflow rate. 


\section{INTRODUCTION}

The project consists of three Phases, with each Phase corresponding to a Project Year. Phase I included an assessment of characteristics associated with produced waters. Phase II involved design, construction, and monitoring of a pilot-scale wetland treatment system. In Phase III, a demonstration system was designed and constructed, and performance in providing onsite treatment of produced water was measured. Rates of treatment in the demonstration system were determined under actual conditions, such as climate and variable water composition and volumes. Data were collected and analyzed for replicating the technology for full-scale treatment at other field sites. In addition, data were collected to compare the technology with other treatment options.

\section{METHODS}

\section{$\underline{\text { Project Management Plan (Task 1) }}$}

A Project Management Plan (PMP) was completed by Clemson University in collaboration with DOE and approved during the first reporting period.

Identify Chemical, Physical, and Risk Characteristics of Produced Waters (Task 2)

The approach in Task 2 involved characterizing produced waters to identify constituents that must be treated to achieve criteria for reuse or discharge. A literature review was conducted on natural gas produced water using published sources and databases from the United States Geological Survey (USGS) and DOE. The data, which include both analytical data and geological source information, were compiled into an Excel spreadsheet. A produced water inventory of these data was created, listing the produced water data by geologic basin and formation. To obtain data on produced waters, we contacted various potential data sources including state geological surveys, state oil and gas boards, and industry personnel.

The characterization included conventional natural gas produced water (NGPW), shale gas produced water (SGPW), coal bed methane produced water (CBMPW), and tight gas sand produced water (TGSPW). Data for some types of unconventional produced water (e.g. SGPW, TGSPW, etc.) can be scarce. Key data of produced water characteristics from shale gas wells were provided by a major producer of shale gas. However, we encountered other operators who were hesitant to provide produced water data from shale gas wells because of their concerns regarding regulatory issues and permitting of new shale gas wells. We received produced water samples from several field sites for analysis.

\section{Determine Reuse and Discharge Criteria and Develop Treatment Performance Goals (Task 3)}

Post-treatment options for produced waters were assessed and compared by examining reuse and discharge criteria. These criteria for reuse were compiled from appropriate published sources, 
including USEPA publications and NPDES (National Pollutant Discharge Elimination System) permits. Reuse criteria included those for livestock, irrigation, and discharge to receiving aquatic systems.

\section{Design and Construct Pilot-Scale Constructed Wetland Treatment Systems (Task 4)}

Pilot-scale constructed wetland treatment systems were designed and constructed at Clemson University based on fundamental reactions and processes needed to treat (transfer and transform) the targeted constituents identified in the previous tasks. Four separate systems were constructed for representative constituents with specific water compositions selected based on the results of Tasks 2 and 3.

\section{Measure Treatment Performance of Pilot-Scale Constructed Wetland Systems (Task 5)}

Performance from the four pilot-scale constructed wetland treatment systems designed and constructed during Task 4 was measured using sampling methods that allow for the determination of both the rate and extent of treatment. In conjunction with performance parameters, explanatory parameters were monitored to determine the conditions needed for efficient removal.

\section{Assess Pilot-Scale Performance (Task 6)}

In Task 6, rate coefficients, extents, and efficiencies for pilot-scale treatment were calculated using data collected during Task 5. Measured performance parameters were compared with treatment goals to assess the success of each pilot-scale system.

\section{$\underline{\text { Design and Construct Demonstration Constructed Wetland Treatment System (Task 7) }}$}

Data from Task 6, including rate coefficients, extents, and efficiencies, were used in Task 7 to design a demonstration constructed wetland treatment system (CWTS). In addition, specific experiments in support of designing CWTSs were performed using pilot-scale systems to gain a better understanding of conditions affecting treatment pathways and hybrid design strategies that can be incorporated into constructed wetland treatment. The experiments included investigating the following: addition of zeolite for sorption, biogeochemical conditions and processes, effect of water depth, effect of seasonal variation, addition of a nutrient amendment, incorporation of an oil/water separator, and evapotranspiration.

The demonstration CWTS was constructed to treat CBMPW from the Black Warrior basin in central Alabama. The CWTS was designed with sequential oxidizing and reducing cells to treat constituents of concern, including ammonia, barium, cadmium, iron, manganese, nitrate, and selenium, identified through sample analyses. 
Measure Treatment Performance of Demonstration Constructed Wetland System (Task 8)

Performance of the demonstration wetland treatment system designed and constructed during Task 7 was monitored using sampling and analytical methods that allow for calculation of removal efficiency, removal rate, and removal extent of constituents of concern. Explanatory parameters were also measured to ensure conditions were suitable for effective treatment.

\section{Apply Results to Water Management and Technology Replication (Task 9)}

Performance data from the pilot-scale and demonstration CWTSs were applied to evaluating the use of CWTSs at other sites. Suitability of treated waters for reuse or discharge was determined for different areas, and results and treatment costs using CWTSs were compared to other technologies for treating conventional and unconventional produced waters. Finally, the results were applied to developing water management plans and design.

\section{$\underline{\text { Technology Transfer (Task 10) }}$}

Technology transfer activities, including presentations and journal articles, were conducted to disseminate project results and findings.

\section{RESULTS AND DISCUSSION}

\section{$\underline{\text { Project Management Plan (Task 1) }}$}

The revised Project Management Plan was competed, submitted to DOE, and approved during the first reporting period as scheduled.

\section{Identify Chemical, Physical, and Risk Characteristics of Produced Waters (Task 2)}

Based on the data gathered, a wide range of chemical, physical, and risk characteristics occurs among the produced waters being investigated. Targeted constituents in the various produced waters include anions, cations (including metals), organics, dissolved solids, and suspended solids. Data on the water volumes produced are scarce in the sources investigated.

Many of the conventional gas produced water data were obtained from published sources for basins that co-produce both oil and natural gas. The compilation contains records for 635 samples of co-produced water from 36 geologic basins. Of these 635 samples, all have organics data and 223 have metals data.

The produced water data for unconventional gas include tight gas sand, shale, and coal bed formations. Data were compiled from 377 coal bed methane (CBM) records from several geologic basins, including Uinta, Black Warrior, Raton, Powder River, and Arkoma. Of these 377 samples, all have records for metals, 88 have records for organics, and 243 have records for 
nitrogen-associated compounds (i.e. nitrate, nitrite, and ammonia). Data from 541 shale gas records were compiled from four basins; of these, 499 have metals data. Data from 137 tight gas sand records were compiled from eight basins; of these, one has metals data and one has organics data.

\section{Characterization}

\section{Produced Water}

Produced water is generated during processes such as fossil fuel extraction and fossil energy production. As water trapped in sediments and sedimentary rocks moves through the subsurface, it tends to accumulate a unique array of ionic constituents (Tucker, 2001). These constituents may then occur in waters produced with oil and gas. Based upon their origins, produced waters can be categorized into two different classes: conventional produced waters and unconventional produced waters. Conventional produced waters are generally considered to be waters that are produced during traditional fossil fuel extraction. Unconventional produced waters are waters produced during extraction of non-traditional fossil fuels or during industrial processes. Conventional produced waters include: oilfield produced water (OFPW), natural gas produced water (NGPW), and natural gas storage produced water (NGSPW). Unconventional produced waters include: coal bed methane produced water (CBMPW), tight gas sand produced water (TGSPW), shale gas produced water (SGPW), oil sand produced water, industry cooling produced water, and industry sluicing produced water. Some of these waters are brought to the surface during recovery of fossil fuels from underground deposits, and others have origins on the surface and are used by industry as process water or for internal cooling and sluicing operations for compliance with environmental regulations. Produced water can range from fresh to hypersaline; for further characterization, produced waters are compared to surface waters with regard to mean constituent concentrations (Table 1).

As global fresh water resources are depleting, consumptive use of fresh water is increasing, as well as costs associated with disposal of produced water. The notion of produced water as a valuable resource through reuse is becoming a more feasible and necessary practice. With adequate characterization and treatment of produced water, applications may be implemented for either internal reuse (e.g. cooling, sluicing, etc.) or external reuse (e.g. surface discharge, ground water recharge, irrigation, etc.) for consumptive and/or non-consumptive purposes. Consumptive reuse of produced water is defined as the reuse of water in a manner that removes it from a source and releases it in a different location that cannot recharge the original system. Nonconsumptive reuse is defined as removing water from a source and returning it to the same source, thus replacing what was taken (Agarwal et al., 2000). Non-consumptive reuse is clearly a more desirable practice; however, if the water returning to the originating system is not adequately treated, the environmental repercussions to the receiving system may outweigh the benefit of the influx of water (Agarwal et al., 2000).

Produced waters contain both inorganic and organic constituents. The major inorganic constituents include chloride, sodium, calcium, magnesium, potassium, mercury, iron, lead, and selenium as well as many other metals, metalloids and non-metals. Produced waters can contain many organic constituents as well, such as: oil and grease, phenols, $n$-alkanes, and low 
molecular-weight aromatic hydrocarbons such as benzene, toluene, ethylbenzene, and xylene (BTEX) (Veil et al., 2004). Depending on the designated reuse for the produced water, both inorganic and organic constituents may be targeted for treatment.

Table 1. Mean constituent concentrations ( $\mathrm{mg} / \mathrm{L})$ for fresh, brackish, saline and hypersaline surface waters

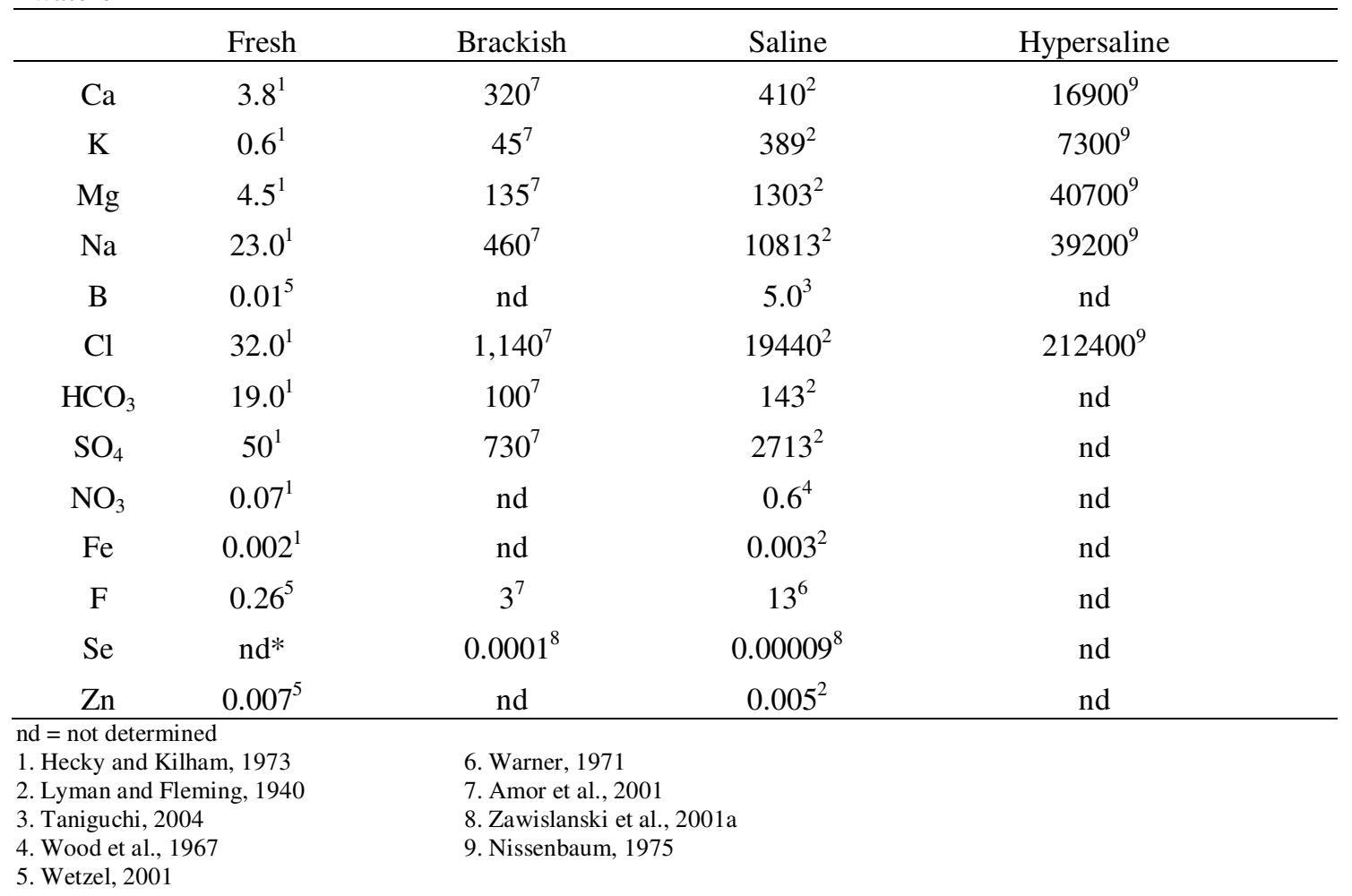

\section{Chemical Characterization}

Produced waters typically contain a range of concentrations of many constituents of concern (COCs), including chlorides, hydrocarbons, metals, and metalloids (Ray and Engelhardt, 1992; Johnson et al., 2008a). Chemical characteristics measured for produced water also include: total dissolved solids (TDS), $\mathrm{pH}$, hardness, alkalinity, five-day biochemical oxygen demand ( $\mathrm{BOD}_{5}$ ), chemical oxygen demand (COD), conductivity, and dissolved oxygen concentration (DO) (Table 2). The predominant constituents in produced water are dissolved salts (Fillo et al., 1992) expressed as salinity, conductivity, or TDS (Veil et al., 2004). The majority of these salts consist of sodium chloride; however, the ionic composition of produced water can range widely (Fillo et al., 1992). Cations found in produced water commonly include sodium, calcium, magnesium, and potassium; anions present often include chloride, sulfate and bicarbonate (Fillo et al., 1992). The inorganics (i.e. metals, metalloids, and nonmetals) present in produced water can include any or all of the following: barium, iron, manganese, potassium, strontium, lithium, zinc, cadmium, cobalt, copper, lead, mercury, chromium, nickel, and several others (Fillo et al., 1992). Organic constituents can be dissolved or dispersed in the produced water and belong to one of 
the following classes: aliphatic hydrocarbons, phenols, carboxylic acids (e.g. naphthenic acids), and low-molecular weight aromatic compounds (Stephenson, 1992). Dispersed organic constituents (e.g. oil and grease) often consist of small suspended droplets (Stephenson, 1992). Gases (e.g. carbon dioxide, hydrogen sulfide, methane) can be formed by chemical reactions within the produced water. Naturally occurring radioactive material (NORM) originates in some geological formations and can be brought to the surface with produced water. NORM in produced water includes radium-226 and radium-228, which are derived from the radioactive decay of uranium and thorium associated with formations in the hydrocarbon reservoir (Utvik, 1999). As the water approaches the surface, temperature changes can cause radioactive elements to precipitate. The resulting scales and sludges may accumulate in water separation systems (Veil et al., 2004).

In addition to chemicals that occur naturally in produced water, chemicals may be added during production processes. These production chemicals are added to treat or prevent operational problems (e.g. scaling, biofouling, and corrosion). Production treatment chemicals include scale inhibitors, corrosion inhibitors, biocides, emulsion breakers, and water treatment chemicals (coagulants/flocculants) (Stephenson, 1992).

\section{Physical Characterization}

Physical characteristics of produced water include color, turbidity, temperature, and odor. The physical characteristics of produced water are often due to reservoir geology, hydrocarbon composition, geographical location, and water injection history (VWS, 2009).

\section{Biological Characterization}

Characterization of a specific produced water for a potential reuse may include a biological component as well. Biological characterization would include questions such as: "is it toxic?" and "how toxic is it?" These two questions of aquatic life sustainability are answered in different ways. To determine if toxicity exists in a specific water, a range-finding toxicity experiment can be conducted to determine a range of concentrations of COCs that elicit a response in a test organism with regard to an exposure (Table 3). Once the range of critical concentrations has been narrowed, a toxicity identification evaluation (TIE) may be conducted to discern the specific concentrations at which selected COCs are toxic. Three parameters are typically measured in toxicity testing: mortality, growth, and reproduction.

There are several methods of aquatic and terrestrial toxicity testing that can evaluate the ability of a specific water to support a particular organism and its survival, growth, or reproduction. Phytotoxicity evaluation is of special concern in areas where fresh water is scarce and toxic constituents in a water may prohibit its use for irrigation. Phytotoxicity testing evaluations can be conducted in a fashion similar to the aquatic animal toxicity evaluations, with the exception that seed germination or sustained crop growth over time is the toxicity identifier instead of mortality or failure of either growth or reproduction. 
Table 2. Analytical methods

\begin{tabular}{|c|c|c|}
\hline Parameter & Method & Detection Limit \\
\hline Turbidity & Closed reflux colorimetery (Standard Methods: 2130 B) & $0.1 \mathrm{NTU}$ \\
\hline $\mathrm{pH}$ & Direct Instrumentation: Orion Model 420A & 0.01 S.U. \\
\hline Conductivity & Direct Instrumentation: YSI 30 & $0.1 \mu \mathrm{S} / \mathrm{cm}$ \\
\hline Alkalinity & Standard Methods: 2320 B & $2 \mathrm{mg} / \mathrm{L}$ as $\mathrm{CaCO} 3$ \\
\hline Hardness & Standard Methods: 2340 B & $2 \mathrm{mg} / \mathrm{L}$ as $\mathrm{CaCO} 3$ \\
\hline $\mathrm{DO}^{1}$ & Direct Instrumentation: YSI Model 52 & $0.1 \mathrm{mg} / \mathrm{L}$ \\
\hline $\mathrm{COD}^{2}$ & Closed reflux colorimetery (HACH- modified from Standard Methods: 5220D) & $3 \mathrm{mg} / \mathrm{L}$ \\
\hline $\mathrm{BOD}_{5}{ }^{3}$ & Standard Methods: 5210 B & $0.1 \mathrm{mg} / \mathrm{L}$ \\
\hline $\mathrm{TSS}^{4}$ & Standard Methods: 2540 D & $0.1 \mathrm{mg} / \mathrm{L}$ \\
\hline $\operatorname{TDS}^{5}$ & Standard Methods: $2540 \mathrm{C}$ & $0.1 \mathrm{mg} / \mathrm{L}$ \\
\hline Ammonia & Direct Instrumentation: Orion Model 95-12 (Standard Methods: 4500-D) & $0.03 \mathrm{mg} / \mathrm{L}$ \\
\hline Nitrate & Standard Methods: HACH-modified from Standard Methods: 4500-NO3 & $0.01 \mathrm{mg} / \mathrm{L}$ \\
\hline Chloride & HACH tritration method & $10-10,000 \mathrm{mg} / \mathrm{L}$ \\
\hline Toxicity & Ceriodaphnia dubia and Pimephales promelas & \\
\hline Oil and grease & EPA Method 1664 A (Environmental Express StepSaver Modification) & $\sim 5 \mathrm{mg} / \mathrm{L}$ \\
\hline & & $(\mathrm{mg} / \mathrm{L})$ \\
\hline Barium & Inductively Coupled Plasma-Atomic Emissions Spectrometry (ICP-AES): 200.7 & 0.0023 \\
\hline Iron & Inductively Coupled Plasma-Atomic Emissions Spectrometry (ICP-AES): 200.7 & 0.0062 \\
\hline Manganese & Inductively Coupled Plasma-Atomic Emissions Spectrometry (ICP-AES): 200.7 & 0.0014 \\
\hline Nickel & Inductively Coupled Plasma-Atomic Emissions Spectrometry (ICP-AES): 200.7 & 0.015 \\
\hline Arsenic & Inductively Coupled Plasma-Atomic Emissions Spectrometry (ICP-AES): 200.7 & 0.053 \\
\hline Chromium & Inductively Coupled Plasma-Atomic Emissions Spectrometry (ICP-AES): 200.7 & 0.0061 \\
\hline Cobalt & Inductively Coupled Plasma-Atomic Emissions Spectrometry (ICP-AES): 200.7 & 0.007 \\
\hline Copper & Inductively Coupled Plasma-Atomic Emissions Spectrometry (ICP-AES): 200.7 & 0.0054 \\
\hline Lead & Inductively Coupled Plasma-Atomic Emissions Spectrometry (ICP-AES): 200.7 & 0.042 \\
\hline Mercury & Inductively Coupled Plasma-Atomic Emissions Spectrometry (ICP-AES): 200.7 & 0.0025 \\
\hline Silver & Inductively Coupled Plasma-Atomic Emissions Spectrometry (ICP-AES): 200.7 & 0.007 \\
\hline Cadmium & Inductively Coupled Plasma-Atomic Emissions Spectrometry (ICP-AES): 200.7 & 0.0034 \\
\hline Selenium & Inductively Coupled Plasma-Atomic Emissions Spectrometry (ICP-AES): 200.7 & 0.075 \\
\hline Magnesium & Inductively Coupled Plasma-Atomic Emissions Spectrometry (ICP-AES): 200.7 & 0.03 \\
\hline Tin & Inductively Coupled Plasma-Atomic Emissions Spectrometry (ICP-AES): 200.7 & 0.025 \\
\hline Aluminum & Inductively Coupled Plasma-Atomic Emissions Spectrometry (ICP-AES): 200.7 & 0.045 \\
\hline Zinc & Inductively Coupled Plasma-Atomic Emissions Spectrometry (ICP-AES): 200.7 & 0.0018 \\
\hline Sodium & Inductively Coupled Plasma-Atomic Emissions Spectrometry (ICP-AES): 200.7 & 0.029 \\
\hline Calcium & Inductively Coupled Plasma-Atomic Emissions Spectrometry (ICP-AES): 200.7 & 0.03 \\
\hline Potassium & Inductively Coupled Plasma-Atomic Emissions Spectrometry (ICP-AES): 200.7 & 0.7 \\
\hline Boron & Inductively Coupled Plasma-Atomic Emissions Spectrometry (ICP-AES): 200.7 & 0.057 \\
\hline Lithium & Inductively Coupled Plasma-Atomic Emissions Spectrometry (ICP-AES): 200.7 & 0.0037 \\
\hline
\end{tabular}

(USEPA 1991; USEPA, 1994; APHA 2005) 
Table 3. Summary of toxicity measurements for Ceriodaphnia dubia, Daphnia magna, and Pimephales promelas for elements or compounds present in non-traditional waters.

\begin{tabular}{|c|c|c|c|c|c|c|c|c|}
\hline Constituent & Species & Experiment & Effect & $\begin{array}{l}\text { NOEC } \\
(\mathrm{mg} / \mathrm{L})\end{array}$ & $\begin{array}{c}\text { LOEC } \\
(\mathrm{mg} / \mathrm{L})\end{array}$ & $\begin{array}{c}\mathrm{LC}_{50} \\
(\mathrm{mg} / \mathrm{L})\end{array}$ & $\begin{array}{c}\mathrm{IC}_{50} \\
(\mathrm{mg} / \mathrm{L})\end{array}$ & Reference \\
\hline Aluminum & $\mathrm{Cd}$ & $48 \mathrm{~h}$ & Mort & & & 2.88 & & 6,10 \\
\hline Aluminum & $\mathrm{Pp}$ & $96 \mathrm{~h}$ & Mort & & & 4.25 & & 11 \\
\hline Calcium & $\mathrm{Cd}$ & $48 \mathrm{~h}$ & Mort & & & 1830 & & 11 \\
\hline Calcium & $\mathrm{Pp}$ & $96 \mathrm{~h}$ & Mort & & & 4630 & & 11 \\
\hline Magnesium & $\mathrm{Cd}$ & $48 \mathrm{~h}$ & Mort & & & 880 & & 11 \\
\hline Magnesium & $\mathrm{Pp}$ & $96 h$ & Mort & & & 2120 & & 11 \\
\hline Sodium & $\mathrm{Dm}$ & $48 \mathrm{~h}$ & Mort & & & 1820 & & 7,10 \\
\hline Sodium & $\mathrm{Pp}$ & $7 d$ & Mort & & 8000 & & & 11 \\
\hline Chloride & $\mathrm{Cd}$ & $7 d$ & Mort & & & 1042 & & 8 \\
\hline Chloride & $\mathrm{Pp}$ & $7 d$ & Mort & & 8000 & & & 11 \\
\hline Bromine & $\mathrm{Dm}$ & $48 \mathrm{~h}$ & Mort & & 0.46 & & & 9,10 \\
\hline Bromine & $\mathrm{Pp}$ & $7 d$ & Mort & & & 0.78 & & 11 \\
\hline Boron & $\mathrm{Cd}$ & $48 \mathrm{~h}$ & Reprod & & 18 & & & 11 \\
\hline Lithium & $\mathrm{Cd}$ & $7 \mathrm{~d}$ & Mort & & & 0.92 & & 11 \\
\hline Lithium & $\mathrm{Pp}$ & $26 \mathrm{~d}$ & Mort & & & 1.2 & & 11 \\
\hline Barium & $\mathrm{Cd}$ & $48 \mathrm{~h}$ & Mort & & & 13.5 & & 7,10 \\
\hline Manganese & $\mathrm{Cd}$ & $7 \mathrm{~d}$ & Reprod & & & & 9 & 1 \\
\hline Manganese & $\mathrm{Pp}$ & $8 d$ & Mort & & & 38.5 & & 11 \\
\hline Iron & $\mathrm{Cd}$ & $48 \mathrm{~h}$ & Mort & & & 30.06 & & 11 \\
\hline Iron & $\mathrm{Pp}$ & $96 h$ & Mort & & & 2.54 & & 11 \\
\hline Potassium & $\mathrm{Cd}$ & $48 \mathrm{~h}$ & Mort & & & 630 & & 11 \\
\hline Potassium & $\mathrm{Pp}$ & $96 h$ & Mort & & & 880 & & 11 \\
\hline Copper & $\mathrm{Cd}$ & $48 \mathrm{~h}$ & Mort & & & 0.027 & & 3 \\
\hline Copper & $\mathrm{Cd}$ & $7 \mathrm{~d}$ & Reprod & 0.012 & 0.032 & & & 2 \\
\hline Copper & $\mathrm{Pp}$ & $96 \mathrm{~h}$ & Mort & & & 0.49 & & 5 \\
\hline Zinc & $\mathrm{Cd}$ & $48 \mathrm{~h}$ & Mort & & & 0.101 & & 11 \\
\hline Zinc & $\mathrm{Pp}$ & $96 \mathrm{~h}$ & Mort & & & 0.87 & & 5 \\
\hline Fluoride & $\mathrm{Dm}$ & $48 \mathrm{~h}$ & Mort & & & 304 & & 12 \\
\hline Fluoride & $\mathrm{Pp}$ & $96 \mathrm{~h}$ & Mort & & & 205 & & 11 \\
\hline Nitrate & $\mathrm{Cd}$ & $48 \mathrm{~h}$ & Mort & & & 374 & & 4 \\
\hline Nitrate & $\mathrm{Pp}$ & $96 \mathrm{~h}$ & Mort & & & 1341 & & 4 \\
\hline Phosphate & $\mathrm{Pp}$ & $96 \mathrm{~h}$ & Mort & & & 18 & & 11 \\
\hline Strontium & $\mathrm{Dm}$ & $48 \mathrm{~h}$ & Mort & & & 125 & & 7,10 \\
\hline Sulfate & $\mathrm{Cd}$ & $48 \mathrm{~h}$ & Mort & & & 1770 & & 11 \\
\hline Sulfate & $\mathrm{Pp}$ & $96 \mathrm{~h}$ & Mort & & & 2820 & & 11 \\
\hline Lead & $\mathrm{Cd}$ & $24 \mathrm{~h}$ & Mort & 0.051 & 0.099 & & & 11 \\
\hline Arsenic & $\mathrm{Cd}$ & $48 \mathrm{~h}$ & Mort & & & $29.1 \mu \mathrm{M}$ & & 11 \\
\hline Arsenic & $\mathrm{Pp}$ & $96 \mathrm{~h}$ & Mort & & & 9.9 & & 11 \\
\hline Selenium & $\mathrm{Pp}$ & $96 \mathrm{~h}$ & Mort & & & 1.0 & & 11 \\
\hline Chromium & $\mathrm{Pp}$ & $7 \mathrm{~d}$ & Growth & & 6.4 & & & 11 \\
\hline Chromium & $\mathrm{Pp}$ & $96 \mathrm{~h}$ & Mort & & & 37 & & 11 \\
\hline Mercury & $\mathrm{Cd}$ & $48 \mathrm{~h}$ & Mort & & & $.05 \mu \mathrm{M}$ & & 11 \\
\hline Mercury & $\mathrm{Pp}$ & $48 \mathrm{~h}$ & Mort & & & 0.05 & & 11 \\
\hline Cobalt & $\mathrm{Cd}$ & $7 \mathrm{~d}$ & Mort & 0.05 & & & & 11 \\
\hline Cobalt & $\mathrm{Cd}$ & $24 \mathrm{~h}$ & Mort & & & 4.014 & & 11 \\
\hline
\end{tabular}




\begin{tabular}{|c|c|c|c|c|c|}
\hline \multirow{2}{*}{$\begin{array}{l}\text { Cobalt } \\
\text { Cobalt }\end{array}$} & $96 h$ & \multicolumn{2}{|l|}{ Mort } & \multirow[t]{2}{*}{12.6} & 11 \\
\hline & $\mathrm{Pp}$ & Mort & 1.232 & & 11 \\
\hline \multicolumn{2}{|c|}{ 1. Lasier et al., 1999.} & \multicolumn{2}{|c|}{ 5. Buhl, 1998.} & \multicolumn{2}{|c|}{ 9. Dorman et al., 2010.} \\
\hline \multicolumn{2}{|c|}{ 2. Carlson et al., 1986.} & \multicolumn{2}{|c|}{ 6. Soucek et al., 2001.} & \multicolumn{2}{|c|}{ 10. EPA EcoTox, 2009.} \\
\hline \multicolumn{2}{|c|}{ 3. Toussaint, et al., 1995.} & \multicolumn{2}{|c|}{ 7. DeGraeve et al., 1992.} & \multicolumn{2}{|c|}{ 11. Fieser et al., 1986.} \\
\hline \multicolumn{2}{|c|}{ 4. Scott and Crunkilton, 2000.} & \multicolumn{2}{|c|}{ 8. LeBlanc, 1980.} & \multicolumn{2}{|c|}{ 12. Biesinger and Christensen, 1972} \\
\hline
\end{tabular}

\section{Produced Water Types}

Shale Gas Produced Water

SGPW characterization data from several basins were compiled (Table 4). Shale gas produced water has chloride concentrations that range from freshwater $(<5,000 \mathrm{ppm})$ to hypersaline $(>50,000 \mathrm{ppm})$. Metals (e.g. aluminum, iron, and barium) are present in the well prior to fracturing of the shale; other metallic and metalloid constituents (e.g. cobalt, chromium, molybdenum, nickel, vanadium, and arsenic) in shale gas produced water can originate in the fracturing fluids (Sumi, 2008).

\section{Coal-bed Methane Produced Water}

Coal bed methane (CBM) may be produced from water-saturated coal seams. This process requires dewatering of coal in order to mobilize methane (Rice and Nuccio, 2000). The water extracted from the formation is brought to the surface as CBMPW (Rice and Nuccio, 2000; Veil et al., 2004). Because of the amount of water generated during dewatering and the rapid increase in CBM exploration and production, management of these waters is a concern (Nelson, 1999; Rice and Nuccio, 2000). Some of the major CBM producing basins include the Powder River Basin in Montana and Wyoming, the Raton Basin in Colorado and New Mexico, the Black Warrior Basin in Alabama, and the Uinta Basin in Utah (Rice and Nuccio, 2000; Benko and Drewes, 2008). The chemistry of the waters produced may vary due to the depth and depositional environment (Rice and Nuccio, 2000). CBM waters typically contain dissolved inorganic elements and dissolved organic compounds (Rice and Nuccio, 2000; Benko and Drewes, 2008).

To identify the water characteristics of CBMPW, several sources were reviewed including papers by the USGS on the Powder River Basin, the Arkoma Basin, and the Ferron Sand of the Uinta Basin, as well as CBMPW water chemistry reports from the Colorado Oil and Gas Conservation Commission (COGCC), the Geological Survey of Alabama (GSA), and the Montana Groundwater Information Center (GWIC).

In total, 377 records of CBMPW composition exist in our compilation consisting of data from the Powder River, Uinta, Raton, Black Warrior, and Arkoma basins. The data are sufficient to be used to identify specific COCs for CBMPW based on specific reuse criteria. The characteristics include general water chemistry parameters such as $\mathrm{pH}$, temperature, conductivity, TDS, TSS, alkalinity, and hardness, as well as major cations and anions, trace metals, nitrogen species, and organic carbon. Statistical analyses have been conducted on the data to determine the range and 
mean of each parameter (Table 5).

CBMPW production quantity data were recorded for each basin. The Powder River basin yields approximately 370,000 cubic meters per day $\left(\mathrm{m}^{3} / \mathrm{d}\right)$ of produced water, the Uinta Basin produces approximately $42,000 \mathrm{~m}^{3} / \mathrm{d}$, the Raton Basin produces approximately $19,000 \mathrm{~m}^{3} / \mathrm{d}$, and the Black Warrior Basin produces approximately 27,000 $\mathrm{m}^{3} / \mathrm{d}$ (Rice and Nuccio, 2000; Benko and Drewes, 2008). Water production rates from individual wells may vary greatly based upon drilling methods, geologic location, and age of the well (Rice and Nuccio, 2000; Veil et al., 2004; Benko and Drewes, 2008).

\section{Oil Field Produced Water}

In addition to natural gas produced waters, constituents of some specific oilfield produced waters (OFPWs) were examined. The data used were provided by Chevron and are pertinent to Phases II and III of the project. Constituents in OFPW depend on depositional environment of the reservoir, trapping mechanism, and adjacent rock units within the basin. Produced waters from oil fields may contain groundwater or seawater that is injected to maintain reservoir pressure (Veil et al., 2004). Data included concentrations for cations and anions, oil and grease, specific polycyclic aromatic hydrocarbons (PAHs), and general water quality parameters ( $\mathrm{pH}$, total suspended solids, total dissolved solids, alkalinity, conductivity, dissolved oxygen, and hardness) (Table 6). 
Table 4. Characterization of shale gas produced water ( $\mathrm{mg} / \mathrm{L}$ except as indicated) $(\mathrm{n}=541)$

\begin{tabular}{|c|c|c|}
\hline Constituents & Mean & Range \\
\hline Temperature $\left({ }^{\circ} \mathrm{C}\right)$ & 25 & \\
\hline pH (S.U.) & 6.124 & $1.21-8.36$ \\
\hline Alkalinity & & $160-188$ (total as $\mathrm{CO}_{3}$ ) \\
\hline TOC & & $.05-10 \%$ \\
\hline Salinity & 190392 & $19-337900$ \\
\hline TDS & 76130.6 & $1724-228971$ \\
\hline TSS & & $200-15,000$ \\
\hline $\mathrm{Al}$ & 126.5 & $0-5290$ \\
\hline B & 0.45 & $0.12-2.18$ \\
\hline $\mathrm{Ba}$ & 318.09 & $0-4370$ \\
\hline $\mathrm{Br}$ & 684.76 & $0-10600$ \\
\hline $\mathrm{Ca}$ & 14856 & $0.65-83950$ \\
\hline $\mathrm{Cl}$ & 84273 & $48.97-212700$ \\
\hline $\mathrm{Cu}$ & 0.795 & $0-15$ \\
\hline $\mathrm{F}$ & 3.06 & $0-33$ \\
\hline $\mathrm{Fe}$ & 137.8 & $0-2838$ \\
\hline $\mathrm{HCO}_{3}$ & 244.36 & $0-4000$ \\
\hline I & 20.75 & $0.8-105$ \\
\hline K & 1091.99 & $0.21-5490$ \\
\hline $\mathrm{Li}$ & 39.39 & $0-611$ \\
\hline $\mathrm{Mg}$ & 1830.6 & $1.08-25340$ \\
\hline $\mathrm{Mn}$ & 7.73 & $0-96.5$ \\
\hline $\mathrm{Na}$ & 34672.2 & $10.04-204302$ \\
\hline $\mathrm{NO}_{3}$ & 226.1 & $0-2670$ \\
\hline $\mathrm{PO}_{4}$ & 0.276 & $0-5.3$ \\
\hline $\mathrm{SiO}_{2}$ & 52.57 & $0.03-5020$ \\
\hline $\begin{array}{l}\mathrm{SO}_{4} \\
\mathrm{Sr}\end{array}$ & $\begin{array}{l}241.43 \\
594.23\end{array}$ & $\begin{array}{c}0-3663 \\
0.03-13100\end{array}$ \\
\hline $\mathrm{Zn}$ & 1.43 & $0-20$ \\
\hline
\end{tabular}

* $\mathrm{As}_{\mathrm{CaCO}}$

(McIntosh et al., 2002; Hill, 2002; GPRI, 2003; Crowe, 2007; Burnett, 2007;

Dodds, 2008; Sumi, 2008; Hill et al., 2008); Carter (PA Geol. Surv., pers.

comm., 2009) 
Table 5. Characterization of coal bed methane produced water

\begin{tabular}{|c|c|c|c|}
\hline Constituent & Units & Range & Mean \\
\hline $\mathrm{pH}$ & S.U. & $6.56-9.87$ & - \\
\hline Temperature & ${ }^{\circ} \mathrm{C}$ & $1.2-41.7$ & 18.71 \\
\hline Conductivity & $\mathrm{mS} / \mathrm{cm}$ & $94.8-145000$ & 4419.57 \\
\hline Total Dissolved Solids & $\mathrm{mg} / \mathrm{L}$ & $270.18-114000$ & 5197.25 \\
\hline Alkalinity & $\mathrm{mg} / \mathrm{L}$ as $\mathrm{CaCO} 3$ & 54.9-9450 & 1858.84 \\
\hline $\mathrm{Ba}$ & $\mathrm{mg} / \mathrm{L}$ & $<0.01-190$ & 7.04 \\
\hline $\mathrm{Br}$ & $\mathrm{mg} / \mathrm{L}$ & $<0.002-300$ & 37.8 \\
\hline $\mathrm{Ca}$ & $\mathrm{mg} / \mathrm{L}$ & $0.8-5870$ & 53.63 \\
\hline $\mathrm{Cl}$ & $\mathrm{mg} / \mathrm{L}$ & $0.7-70100$ & 845.96 \\
\hline $\mathrm{F}$ & $\mathrm{mg} / \mathrm{L}$ & $<0.05-15.22$ & 2.52 \\
\hline $\mathrm{Fe}$ & $\mathrm{mg} / \mathrm{L}$ & $<0.002-220$ & 2.74 \\
\hline K & $\mathrm{mg} / \mathrm{L}$ & $0.3-186$ & 10.63 \\
\hline $\mathrm{Mg}$ & $\mathrm{mg} / \mathrm{L}$ & $0.2-1830$ & 34.59 \\
\hline $\mathrm{Na}$ & $\mathrm{mg} / \mathrm{L}$ & $8.8-34100$ & 1183.08 \\
\hline $\mathrm{Si}$ & $\mathrm{mg} / \mathrm{L}$ & $<0.1-49.8$ & 9.9 \\
\hline $\mathrm{Sr}$ & $\mathrm{mg} / \mathrm{L}$ & $0.032-565$ & 16.08 \\
\hline Sodium Absorption Ratio & - & $5.7-32$ & 11.67 \\
\hline Ammonia & $\mathrm{mg} / \mathrm{L}$ & $1.05-59$ & 3.53 \\
\hline Nitrate & $\mathrm{mg} / \mathrm{L}$ & $<0.002-18.7$ & 1.22 \\
\hline Phosphate & $\mathrm{mg} / \mathrm{L}$ & $<0.050-1.5$ & 0.23 \\
\hline Sulfates & $\mathrm{mg} / \mathrm{L}$ & $<0.01-5590$ & 335.14 \\
\hline Total Organic Carbon & $\mathrm{mg} / \mathrm{L}$ & $1-100$ & 9.50 \\
\hline $\mathrm{Ag}$ & $\mu \mathrm{g} / \mathrm{L}$ & $<0.5-375$ & 63.10 \\
\hline $\mathrm{Al}$ & $\mu \mathrm{g} / \mathrm{L}$ & $0.2-1240$ & 201.4 \\
\hline As & $\mu \mathrm{g} / \mathrm{L}$ & $0.1-60$ & 3.21 \\
\hline B & $\mu \mathrm{g} / \mathrm{L}$ & $1.6-2400$ & 151.36 \\
\hline $\mathrm{Be}$ & $\mu \mathrm{g} / \mathrm{L}$ & $<0.1$ & $<0.1$ \\
\hline $\mathrm{Bi}$ & $\mu \mathrm{g} / \mathrm{L}$ & $19-32$ & 24.45 \\
\hline $\mathrm{Cd}$ & $\mu \mathrm{g} / \mathrm{L}$ & $<0.1-10$ & 4.75 \\
\hline $\mathrm{Ce}$ & $\mu \mathrm{g} / \mathrm{L}$ & $<10.0$ & $<10.0$ \\
\hline Co & $\mu \mathrm{g} / \mathrm{L}$ & $<0.1-0.729$ & 0.17 \\
\hline $\mathrm{Cr}$ & $\mu \mathrm{g} / \mathrm{L}$ & $<1.0-53$ & 11.49 \\
\hline Cs & $\mu \mathrm{g} / \mathrm{L}$ & $<0.1-0.78$ & 0.18 \\
\hline $\mathrm{Cu}$ & $\mu \mathrm{g} / \mathrm{L}$ & $<0.2-60$ & 8.75 \\
\hline $\mathrm{Hg}$ & $\mu \mathrm{g} / \mathrm{L}$ & $<0.005-0.4$ & 0.21 \\
\hline $\mathrm{La}$ & $\mu \mathrm{g} / \mathrm{L}$ & $<10.0$ & $<10.0$ \\
\hline $\mathrm{Li}$ & $\mu \mathrm{g} / \mathrm{L}$ & $0.21-6880$ & 146.25 \\
\hline $\mathrm{Mn}$ & $\mu \mathrm{g} / \mathrm{L}$ & $<2.0-5400$ & 59.12 \\
\hline Mo & $\mu \mathrm{g} / \mathrm{L}$ & $<0.5-100$ & 28.84 \\
\hline $\mathrm{Ni}$ & $\mu \mathrm{g} / \mathrm{L}$ & $0.304-203$ & 19.71 \\
\hline $\mathrm{P}$ & $\mu \mathrm{g} / \mathrm{L}$ & $<50-94$ & 84.0 \\
\hline $\mathrm{Pb}$ & $\mu \mathrm{g} / \mathrm{L}$ & $<0.1-585$ & 117.67 \\
\hline $\mathrm{Rb}$ & $\mu \mathrm{g} / \mathrm{L}$ & $4.1-38.2$ & 11.79 \\
\hline $\mathrm{Sb}$ & $\mu \mathrm{g} / \mathrm{L}$ & $<0.1-950$ & 575.0 \\
\hline $\mathrm{Sc}$ & $\mu \mathrm{g} / \mathrm{L}$ & $<0.1-3$ & 1.34 \\
\hline $\mathrm{Se}$ & $\mu \mathrm{g} / \mathrm{L}$ & $<0.1-70$ & 7.44 \\
\hline
\end{tabular}




\begin{tabular}{llcc}
$\mathrm{Sn}$ & $\mu \mathrm{g} / \mathrm{L}$ & $<0.1-680$ & 89.58 \\
$\mathrm{Th}$ & $\mu \mathrm{g} / \mathrm{L}$ & $<20.0$ & $<20.0$ \\
$\mathrm{Ti}$ & $\mu \mathrm{g} / \mathrm{L}$ & $<1.0-45$ & 12.71 \\
$\mathrm{Tl}$ & $\mu \mathrm{g} / \mathrm{L}$ & $<0.1-0.34$ & 0.34 \\
$\mathrm{U}$ & $\mu \mathrm{g} / \mathrm{L}$ & $<0.5-50$ & 12.82 \\
$\mathrm{~V}$ & $\mu \mathrm{g} / \mathrm{L}$ & $0.19-59$ & 13.24 \\
$\mathrm{~W}$ & $\mu \mathrm{g} / \mathrm{L}$ & $<20.0$ & $<20.0$ \\
$\mathrm{Y}$ & $\mu \mathrm{g} / \mathrm{L}$ & $<10.0$ & $<10.0$ \\
$\mathrm{Zn}$ & $\mu \mathrm{g} / \mathrm{L}$ & $0.02-590$ & 39.96 \\
$\mathrm{Zr}$ & $\mu \mathrm{g} / \mathrm{L}$ & $0.21-131$ & 27.68 \\
\hline (Hunter and Moser, 1990; Rice, 1999; Rice et al., 2000; ESN Rocky Mountain, 2003; Thordsen et al. \\
2007; Montana GWIC, 2009)
\end{tabular}

Table 6. Characterization of oil field produced water. $n=$ number of samples.

\begin{tabular}{lcccr}
\hline Parameter & Mean $(\mathrm{mg} / \mathrm{L})$ & Minimum $(\mathrm{mg} / \mathrm{L})$ & Maximum $(\mathrm{mg} / \mathrm{L})$ & $\mathrm{n}$ \\
\hline Alkalinity $\left(\mathrm{mg} / \mathrm{L}\right.$ as $\left.\mathrm{CaCO}_{3}\right)$ & 326.2 & 300 & 380 & 13 \\
Barium & 4.1 & 0.07 & 7.4 & 7 \\
Bicarbonate & 704.7 & 433 & 976 & 2 \\
Calcium & 164.19 & 2.5 & 300 & 20 \\
Carbonate & 14.6 & 14.6 & 14.6 & 1 \\
Chloride & 12.33 & 5 & 48 & 15 \\
Conductivity ( $\mu$ mhos) & 1153.5 & 838 & 1469 & 2 \\
Iron (III) & 54.9 & 24.4 & 171 & 5 \\
Iron (soluble) & 0.14 & 0.1 & 0.5 & 13 \\
Hardness $\left(\mathrm{mg} / \mathrm{L}\right.$ as $\left.\mathrm{CaCO}_{3}\right)$ & 10.9 & 5 & 20 & 13 \\
Magnesium & 3.7 & 1.05 & 8.7 & 7 \\
Manganese & 6.3 & 1.4 & 8.1 & 5 \\
Nickel & 7.7 & 2.7 & 9.5 & 5 \\
Nitrate & 1.5 & 1 & 2 & 2 \\
Oil and grease & 92.0 & 92 & 92 & 1 \\
Dissolved oxygen & 0.00014 & 0.0001 & 0.0002 & 13 \\
pH (standard units) & 7.78 & 7.73 & 8.34 & 15 \\
Potassium & 10.5 & 1.6 & 42.6 & 7 \\
Silicon & 6.0 & 1.4 & 13.2 & 7 \\
Sodium & 115.3 & 8.8 & 339 & 7 \\
Strontium & 1.3 & 0.05 & 2.2 & 7 \\
Sulfate & 2.5 & 2 & 3 & 2 \\
TDS & 1037.0 & 704 & 1370 & 2 \\
Tin & 0.7 & 0.5 & 1.3 & 5 \\
Zinc & 12.8 & 6.3 & 17.4 & 5 \\
\hline
\end{tabular}

Conventional Natural Gas Produced Water

Conventional natural gas is extracted from underground formations typically comprised of permeable sandstone, and can be produced from other rock formations (e.g. limestone). Conventional natural gas accounts for approximately $61 \%$ of total natural gas production in the nation (EIA, 2008). During the extraction process, large quantities of formation water may be 
brought to the surface as NGPW. Because of the extensive nature of conventional natural gas production and the inherent water production, the management of these NGPWs presents a challenge. Characteristics of NGPWs are listed in Table 7.

\section{Tight Gas Sand Produced Water}

Tight gas sand is a low-permeability (less than 0.1 millidarcy) reservoir requiring natural and/or induced well stimulation for acceptable rates of gas flow (Kuuskraa 2004). Low-permeability reservoirs have the characteristics of small pore throats, strong water wettability, and strong capillary inhibition effect; thus they are very susceptible to damage during drilling, completion, production, and stimulation, which can lead to low production rates (Bennion et al. 1995). Chloride concentrations from the 137 records of TGSPW correspond to brackish to saline waters. Mean, maximum, and minimum concentrations of constituents are listed in Table 8.

Table 7. Characteristics of conventional natural gas produced water (units are same as in Table 5)

\begin{tabular}{|c|c|c|c|c|c|}
\hline Constituent & Min & Max & Constituent & Min & Max \\
\hline $\mathrm{pH}^{1}$ & 4.4 & 7.0 & Iron $^{1}$ & bdl & 1100 \\
\hline $\mathrm{pH}^{2}$ & 3.1 & 6.47 & Iron $^{2}$ & 39 & 680 \\
\hline Conductivity & 4200 & 180000 & Lead $^{2}$ & $<0.2$ & 10.2 \\
\hline (umhos/cm) & 136000 & 586000 & Lithium $^{2}$ & 18.6 & 235 \\
\hline Alkalinity $^{2}$ & 0 & 285 & Magnesium $^{1}$ & 0.9 & 4300 \\
\hline $\operatorname{TDS}^{1}$ & 2600 & 310000 & Magnesium² & 1300 & 3900 \\
\hline $\operatorname{TDS}^{2}$ & 139000 & 360000 & Manganese $^{1}$ & 0.045 & 6.5 \\
\hline $\mathrm{TSS}^{2}$ & 8 & 5484 & Nickel $^{1}$ & bdl & 0.02 \\
\hline $\mathrm{BOD}_{5}{ }^{1}$ & 75 & 2870 & Nickel $^{2}$ & $<0.08$ & 9.2 \\
\hline $\mathrm{COD}^{1}$ & 2600 & 120000 & Potassium $^{2}$ & 149 & 3870 \\
\hline Aluminum ${ }^{1}$ & bdl & 0.4 & Silver $^{2}$ & 0.027 & 7 \\
\hline Aluminum $^{2}$ & $<0.50$ & 83 & Sodium $^{1}$ & 520 & 45000 \\
\hline Arsenic $^{1}$ & 0.004 & 1 & Sodium $^{2}$ & 37500 & 120000 \\
\hline Arsenic $^{2}$ & $<0.005$ & 1.51 & Strontium $^{1}$ & & 6200 \\
\hline Barium $^{1}$ & bdl & 26 & Sulfate $^{2}$ & $<1.0$ & 47 \\
\hline Barium $^{2}$ & 9.65 & 1740 & Sulfide $^{1}$ & bdl & 19 \\
\hline Boron $^{1}$ & bdl & 56 & $\operatorname{Tin}^{1}$ & bdl & 1.1 \\
\hline Bromide $^{2}$ & 150 & 1149 & Zinc $^{1}$ & bdl & 0.022 \\
\hline Cadmium $^{1}$ & bdl & 0.015 & Zinc $^{2}$ & $<0.02$ & 5 \\
\hline Cadmium $^{2}$ & $<0.02$ & 1.21 & $\mathrm{TOC}^{1}$ & 67 & 38000 \\
\hline Calcium $^{1}$ & bdl & 25000 & Surfactants ${ }^{2}$ & 0.08 & 1200 \\
\hline Calcium $^{2}$ & 9400 & 51300 & Benzene $^{1}$ & 1.8 & 6.9 \\
\hline Chloride $^{1}$ & 1400 & 190000 & Benzene $^{3}$ & $<0.01$ & 10.3 \\
\hline Chloride $^{2}$ & 81500 & 167448 & Toluene $^{1}$ & 0.857 & 3.37 \\
\hline Chromium $^{1}$ & bdl & 0.03 & Toluene $^{3}$ & $<0.01$ & 18 \\
\hline Copper $^{1}$ & bdl & 0.02 & Oil/Grease ${ }^{1}$ & 6 & 60 \\
\hline Copper $^{2}$ & $<0.02$ & 5 & Oil/Grease ${ }^{2}$ & 2.3 & 38.8 \\
\hline
\end{tabular}

${ }^{1}$ Fillo and Evans, 1990

${ }^{2}$ USEPA, 2000

${ }^{3}$ Shepard et al., 1992. 
Table 8. Characterization of tight gas sands produced water ( $\mathrm{mg} / \mathrm{L}$ except as indicated) $(\mathrm{n}=137)$

\begin{tabular}{|c|c|c|}
\hline Constituent & Mean & Range \\
\hline $\mathrm{pH}$ (S.U.) & 6.79 & $5-8.6$ \\
\hline Hardness & 780 & \\
\hline Conductivity & 24.42 & \\
\hline Alkalinity & 1424 & \\
\hline Temp $\left({ }^{\circ} \mathrm{C}\right)$ & 22 & \\
\hline Ammonia & 2.74 & \\
\hline Bicarbonate & 666.9 & $10-4040$ \\
\hline Sulfate & 701.98 & $0-8823$ \\
\hline TDS & 130393 & $1588-343300$ \\
\hline TSS & 85.7 & \\
\hline Oil/Grease & 42.0 & \\
\hline $\mathrm{Al}$ & bdl & \\
\hline As & 0.17006 & \\
\hline $\mathrm{Ba}$ & 0.30942 & \\
\hline $\mathrm{Ca}$ & 14342.6 & $3-74185$ \\
\hline $\mathrm{Cd}$ & 0.03689 & \\
\hline $\mathrm{Cl}$ & 79187 & $52-216000$ \\
\hline $\mathrm{Cr}$ & 0.26467 & \\
\hline $\mathrm{Cu}$ & 0.53862 & \\
\hline $\mathrm{Fe}$ & 0.01493 & \\
\hline $\mathrm{K}$ & 983.8 & $5-2500$ \\
\hline $\mathrm{Mg}$ & 1661.5 & $2-8750$ \\
\hline $\mathrm{Mn}$ & 0.52457 & \\
\hline $\mathrm{Na}$ & 33157.7 & $648-80000$ \\
\hline $\mathrm{Ni}$ & 0.12277 & \\
\hline $\mathrm{Pb}$ & 0.48793 & \\
\hline $\mathrm{Se}$ & bdl & \\
\hline $\mathrm{Zn}$ & 0.07615 & \\
\hline
\end{tabular}

Determine Reuse and Discharge Criteria and Develop Treatment Performance Goals (Task 3)

\section{Internal Reuse}

Reinjection

Reinjection into an underground formation is the most commonly used approach for managing produced water (Veil et al., 2004). Although some produced water is injected solely for disposal, most produced water $(71 \%)$ is injected to maintain reservoir pressure and to hydraulically drive oil or gas toward a producing well. The produced water re-injection criteria focus on the concentration of halogenated and non-halogenated solvents decreased to less than $10 \%$ by volume of produced water as well as criteria for metals, metalloids, TSS, TDS, and dissolved oxygen (USEPA 2002a). Physical characteristics are not outlined in the re-injection criteria guidelines. Re-injection standards are governed by the Clean Water Drinking Act (CWDA) and the Underground Injection Control program (UIC). 
Industrial Cooling

According to the U.S. Geological Survey (Hutson et al. 2004), the U.S. power industry withdrew about 136 billion gallons per day of fresh water for power plant cooling; conventional surface and ground water resources are no longer sufficient to meet increasing power plant needs. Produced water represents a large-volume source of water that could potentially serve as makeup water for a power plant (DiFilippo, 2004). Water used for cooling operations should have a low TDS concentration; otherwise, scaling, corrosion, and biofouling of the cooling system may become problematic (WGA, 2006).

Industrial Sluicing

Bottom ash contains non-combustible constituents with traces of combustibles produced during operation of a coal-, oil-, or gas-burning furnace (Sell, 1992; EIA 1995). Ash sluicing systems pump produced water to sluice the bottom ash to waste storage (Prewitt, 1995). To prevent problems with scaling and corrosion of the bottom ash system, water used for industrial sluicing must have low TDS and chloride concentrations.

\section{External Reuse}

Aquaculture

The physical, biological, and chemical reuse criteria for aquaculture are stringent. Water quality determines how well fish will grow and survive in an aquaculture operation (Buttner et al., 1993). Physical and chemical characteristics (e.g. DO, temperature, ammonia concentration) are commonly associated with fish kills, while other characteristics (e.g. pH, alkalinity, hardness, turbidity) can affect the health of a fish population, but are not consistently found to be toxic to the fish (Buttner et al., 1993). Biological characteristics for aquaculture are measured by examining growth, reproduction, and mortality of the aquatic organisms within an aquaculture environment (i.e. laboratory toxicity evaluation).

\section{Livestock}

Water for livestock is an option for reuse of produced water, provided that the COC concentrations are below the EPA water quality criteria for livestock (API, 2004). Chemical analyses can be conducted to determine the COCs and discern which constituents should be targeted for treatment and/or removal. Toxicity tests can be conducted to determine if a specific produced water is toxic, and if so, how toxic it is. Using organisms such as Ceriodaphnia dubia and Pimephales promelas, which are sensitive indicator species, can facilitate determination of toxicities from COCs. 


\section{Drinking}

Drinking water quality criteria are stringent. If produced water were to be reused in this capacity, successful and simultaneous treatment of COCs would likely be necessary. However, achieving drinking water quality with produced water, especially in more arid regions, is desirable. The extent of treatment needed to achieve drinking water standards may vary depending on the type and characteristics of produced water. In order to analyze treatment system effluent to determine if drinking water criteria have been met, a full chemical characterization must be conducted. For evaluation of the specific effluent for potential use as drinking water, a biological and physical characterization must also be conducted, but water that is non-toxic physically or biologically does not always indicate that the water reached drinking water criteria, and vice versa.

\section{Irrigation}

Irrigation presents a feasible reuse option for produced water. Salinity, sodicity, and toxicity are the critical parameters when considering reuse of a specific water for irrigation purposes (Veil et al. 2004). The salinity can be estimated by measuring chloride concentration in the sample.

Biological characterization involves phytotoxicity tests which use seed germination or sustained crop growth over time as an identifier of toxicity. The seed germination or sustained growth test should involve a plant species that is analogous to a crop that may be irrigated with the specific water. A physical characterization focuses on temperature, total suspended solids (TSS), and turbidity.

\section{Groundwater Recharge}

There are specific considerations for characterization of water to be reused for groundwater recharge. Because proximity to the surface is an important factor when determining water quality criteria, physical characterization must include depth of the aquifer to be recharged. With chemical characterization, it is important to examine local mineralogy of the aquifer. Also, certain purposes involved with groundwater recharge call for specific characterizations. For example, if the purpose is to establish a saltwater intrusion barrier in coastal aquifers, then chloride concentration should be considered.

\section{Surface Water Discharge}

Discharging produced water to an existing surface water system presents stringent treatment goals similar to drinking water standards. In accordance with the Clean Water Act (CWA), all point source discharges to federal waters from municipal and industrial facilities must be permitted through the National Pollutant Discharge Elimination System (NPDES). The primary goal of the NPDES is to protect the health of the nation's surface waters by regulating the discharge of contaminants that may present a risk. Regulation is enforced primarily by setting effluent limitations.

NPDES permits are obtained either directly from the U.S. Environmental Protection Agency (USEPA) or from within states that have been given primary enforcement responsibilities (primacy) by the USEPA. Permits are based either on general permitting guidelines established 
for specific industries located in a specified geographic area, or may be specific to a facility. The permitting process involves limits based upon both the potential impacts to the receiving system (water quality standards) as well as technology-based limitations for treating the water defined as effluent limitation guidelines (ELGs). In the absence of applicable ELGs for discharge water, the permit writer may be authorized to use best practical judgment to set an effluent limit. The final permit will include the most stringent limitations established by either the water quality standards or the ELGs including any values determined by best practical judgment.

Water quality standards may be determined using three different methods. In the chemicalspecific approach, chemical analyses are performed on the specific discharge water and the receiving system to determine site-specific potential chemical impacts. In the whole effluent toxicology (WET) approach, the impacts of the discharge water are evaluated by testing the acute and chronic effects of exposure to aquatic organisms. In the biological assessment approach, the biological diversity and integration of a receiving system may be compared to a healthy system to determine the impacts of discharge.

ELGs for oil and gas extraction point source wastes including produced waters may be found in the Code of Federal Regulations (CFR) under 40 CFR Part 435. The ELGs are divided into different categories based upon the level of technology required to meet the guidelines including best conventional pollution control technology (BCT), best practical control technology currently available (BPT), best available technology economically achievable (BAT), and new source performance standards (NSPSs). According to 40 CFR Part 435, oil and grease is the only ELG available. Table 9 lists the levels for oil and grease required by the produced water ELGs based upon the technology levels. Existing dischargers of produced water must meet BCT, BPT, and BAT ELGs while new dischargers must meet the NSPS ELGs. In the case of oil and grease, the most stringent levels found under the BAT and NSPS ELGs are the same and therefore all discharges of produced water must meet the oil and grease ELG of $42 \mathrm{ppm}$ maximum and 29 ppm 30 day maximum.

Table 9. Oil and grease effluent limitation guidelines (ELGs) for produced waters

\begin{tabular}{lcc}
\hline Technology Level & Maximum Level ppm & 30 Day Maximum ppm* \\
\hline BCT & 72 & 48 \\
BPT & 72 & 48 \\
BAT & 42 & 29 \\
NSPS & 42 & 29 \\
\hline *Concentrations of oil and grease may not exceed this value for more than 30 consecutive & \\
days & & \\
40 CFR Part 435 & &
\end{tabular}

\section{Produced Waters and Reuse Purposes}

Reuse criteria for produced waters are listed in Table 10. Specific reuse purposes can be identified for types of produced waters based on the geographic areas of production and water needs associated with those areas. 
Table 10. Selected Reuse Criteria for Produced Waters. From Ayers and Westcot (1994), WDEQ (2001), USEPA (2004), Johnson et al. (2008a)

\begin{tabular}{|c|c|c|c|c|c|}
\hline Parameter $^{1}$ & Irrigation & $\begin{array}{l}\text { Livestock and } \\
\text { Wildlife }\end{array}$ & Aquatic Life & $\begin{array}{c}\text { Surface } \\
\text { Discharge }^{2}\end{array}$ & $\begin{array}{l}\text { Drinking } \\
\text { Water }^{3}\end{array}$ \\
\hline Conductivity (ms/m) & $1700-13000$ & 16000 & - & - & - \\
\hline TDS & - & - & - & 500 & 500 \\
\hline pH (range) (S.U.) & $4.5-9.0$ & $6.5-8.5$ & $6.5-9.5$ & $6.0-9.0$ & $6.5-8.5$ \\
\hline SAR (ratio) & 15 & - & - & - & - \\
\hline Bicarbonate & 520 & - & - & - & - \\
\hline Carbonate & 3 & - & - & - & - \\
\hline Sulfate & 960 & - & - & - & 250 \\
\hline Sulfide & - & - & - & $0.21-1$ & - \\
\hline Nitrite-N & - & 10 & - & - & 1 \\
\hline Nitrate-N & 10 & - & - & - & 10 \\
\hline Ammonia-N & 5 & - & - & - & - \\
\hline Total Nitrogen-N & - & 100 & - & - & - \\
\hline Phosphate-P & 2 & - & - & - & - \\
\hline COD & - & - & - & $170-292$ & - \\
\hline Oil and Grease & 35 & 35 & - & 15 & - \\
\hline $\mathrm{Al}$ & 5 & 5 & 0.1 & - & $.05-.2$ \\
\hline $\mathrm{Ag}$ & - & - & - & - & 0.1 \\
\hline As & 0.1 & 0.2 & 0.15 & - & 0.01 \\
\hline B & $0.5-15$ & 5 & - & - & - \\
\hline $\mathrm{Ba}$ & - & - & 5 & 154 & 2 \\
\hline $\mathrm{Be}$ & 0.1 & 0.1 & - & - & 0.004 \\
\hline $\mathrm{Ca}$ & 400 & - & - & - & - \\
\hline $\mathrm{Cd}$ & 0.01 & 0.05 & 0.002 & - & 0.005 \\
\hline $\mathrm{Cl}$ & 1050 & - & - & 5000 & 250 \\
\hline Co & 0.05 & 1 & - & - & - \\
\hline $\mathrm{Cr}$ & 0.1 & 1 & - & $0.0001-0.1$ & 0.1 \\
\hline $\mathrm{Cu}$ & 0.2 & 0.5 & 0.01 & $0.37-1.0$ & 1 \\
\hline $\mathrm{F}$ & 1 & 2 & - & - & 2 \\
\hline $\mathrm{Fe}$ & 5 & - & 1 & $0.6-9.4$ & 0.3 \\
\hline $\mathrm{Hg}$ & - & 0.01 & - & 0.004 & 0.002 \\
\hline $\mathrm{K}$ & 2 & - & - & - & - \\
\hline $\mathrm{Li}$ & 2.5 & - & - & - & - \\
\hline $\mathrm{Mg}$ & 60 & - & - & - & - \\
\hline $\mathrm{Mn}$ & 0.2 & 0.05 & 1.5 & $1-4.4$ & 0.05 \\
\hline Mo & 0.01 & - & - & - & - \\
\hline $\mathrm{Na}$ & 920 & - & - & - & - \\
\hline $\mathrm{Ni}$ & 0.2 & - & - & 2 & - \\
\hline $\mathrm{Pb}$ & 5 & 0.1 & 2.5 & 0.4 & 0.015 \\
\hline $\mathrm{Sb}$ & - & - & - & - & 0.006 \\
\hline $\mathrm{Se}$ & 0.02 & 0.05 & 0.005 & - & 0.05 \\
\hline Th & - & - & - & - & 0.002 \\
\hline $\mathrm{V}$ & 0.1 & 0.1 & - & - & - \\
\hline $\mathrm{Zn}$ & 2 & 24 & 0.1 & $0.4-5$ & 5 \\
\hline
\end{tabular}

1. All units are $\mathrm{mg} / \mathrm{L}$ unless noted

2. Values obtained from NPDES permits used in the oil and gas industry

3. USEPA secondary standards selected when available 


\section{Shale Gas Produced Water Reuse}

Shale gas is extracted in many areas throughout the United States (e.g. New York, Pennsylvania, Arkansas, Alabama, Texas, and Montana). Shale gas produced water has a variety of potential reuses, including surface discharge and irrigation. Reuse options were determined by location (e.g. latitude, longitude and altitude) and socio-economic needs. Surface water discharge to lakes and rivers after sufficient treatment may be viable in arid regions (e.g. Texas and New Mexico). Irrigation may be a feasible reuse option where suitable water supply is limited because of drought or pollution (Frankenberger and Engberg 1998). Reinjection is commonly used for disposal of SGPW rather than treatment. However, with the increasing need for fresh water sources, treating SGPW for reuse is expected to become more common.

Constituents of concern in SGPW for surface water discharge were identified by comparing the concentrations in SGPW with NPDES surface water discharge permit criteria (Table 11). The COCs are: total suspended solids, total dissolved solids, $\mathrm{pH}$, aluminum, barium, boron, chloride, copper, fluoride, iron, manganese, phosphate, sulfate, and zinc. SGPW COCs for irrigation were determined by comparing the concentrations of constituents of SGPW to USEPA and Food and Agriculture Organization of the United Nations (FAO) irrigation water criteria (Table 12). These COCs are: total suspended solids, total dissolved solids, $\mathrm{pH}$, aluminum, bicarbonate, boron, calcium, chloride, copper, fluoride, iron, lithium, magnesium, manganese, nitrate, phosphate, potassium, sodium, sulfate, and zinc.

\section{Coal Bed Methane Produced Water Reuse}

Beneficial reuse of CBMPW is an attractive option due to: 1) the large quantity of PW generated during coal bed methane extraction; 2) the need for water reuse in coal bed methane producing areas; and 3) the rapid increase in coal bed methane extraction development (Rice and Nuccio 2000). Reuse options may include irrigation, livestock, recreation, aquaculture, industrial cooling and sluicing, wildlife habitat, aquifer recharge, and drinking water (ALL Consulting 2003; Veil et al. 2004). Specific needs may be based on geographic location, climate, water availability, and development. For instance, in rural areas with limited freshwater resources and an adequate growing season, reuse options most likely will include irrigation and livestock rather than industrial reuse.

Based on the locations and climates of the major CBM producing areas, reuse options for CBMPW include irrigation, livestock watering, and aquaculture (Tables 13-15). The selection of multiple guideline sources, including international, federal, and state agencies, promotes adequate determination of reuse guideline levels. Guidelines from Wyoming were selected because the Powder River Basin in Wyoming is responsible for a major portion of CBMPW production (Rice and Nuccio, 2000).

Based on the comparison of CBMPW characterization data to reuse guidelines, the following parameters are considered COCs: conductivity, $\mathrm{pH}, \mathrm{SAR}$, bicarbonate, carbonate, sulfate, nitrate, ammonium, Al, Ba, Ca, Cd, Cl, Cu, F, Fe, K, Li, Mg, Mn, Mo, Na, Ni, Pb, Se, and Zn. The performance of the selected treatment method will be maximized so that the most reuse options may be considered. 
Table 11. NPDES permit criteria and shale gas produced water characteristics for identification of COCs. From USEPA (2004). (All units are mg/L unless noted).

\begin{tabular}{|c|c|c|c|c|c|}
\hline \multirow[t]{2}{*}{ Constituents } & \multicolumn{2}{|c|}{ Shale Gas Produced Water } & \multicolumn{2}{|c|}{ NPDES } & \multirow[t]{2}{*}{$\mathrm{COCs}$} \\
\hline & Min & Max & Min & $\operatorname{Max}$ & \\
\hline Total Suspended Solids & 200 & 15000 & & 45 & Yes \\
\hline Total Dissolved Solids & 1724 & 228971 & 30 & 5000 & Yes \\
\hline pH (S.U.) & 1.21 & 8.36 & $>6$ & $>9$ & Yes \\
\hline Temperature ${ }^{\circ} \mathrm{C}$ & 25 & & & $>40$ & No \\
\hline Alkalinity (as $\mathrm{CaCO}_{3}$ ) & 160 & 188 & & & - \\
\hline Dissolved Oxygen & & & 6 & 17 & - \\
\hline Salinity & 19 & 337900 & & & - \\
\hline Aluminum & 0 & 5290 & & 0.05 & Yes \\
\hline Ammonium-N & & & & & - \\
\hline Arsenic & & & & 0.05 & - \\
\hline Barium & 0 & 4370 & & 154 & Yes \\
\hline Bicarbonate & 0 & 927 & & & - \\
\hline Boron & 0 & 4370 & & 1 & Yes \\
\hline Bromide & 0 & 10600 & & & - \\
\hline Cadmium & & & & 0.0025 & - \\
\hline Calcium & 0.65 & 83950 & & & - \\
\hline Carbonate & & & & & - \\
\hline Chloride & 48.9 & 212700 & & 5000 & Yes \\
\hline Chromium & & & 0.1 & 0.2 & - \\
\hline Cobalt & & & & & - \\
\hline Copper & 0 & 15 & 0.37 & 1 & Yes \\
\hline Fluoride & 0 & 33 & & 30 & Yes \\
\hline Iodine & 0.8 & 105 & & & - \\
\hline Iron & 0 & 2838 & 0.6 & 9.4 & Yes \\
\hline Lead & & & 0.0056 & 0.015 & - \\
\hline Lithium & 0 & 611 & & & - \\
\hline Magnesium & 1.1 & 25340 & & & - \\
\hline Manganese & 0 & 96.5 & 0.05 & 4.4 & Yes \\
\hline Mercury & & & 0.0008 & 0.004 & - \\
\hline Molybdenum & & & & & - \\
\hline Nickel & & & 0.052 & 2 & - \\
\hline Nitrate-N & 0 & 2670 & & & - \\
\hline Phosphate-P & 0 & 5.3 & & 1 & Yes \\
\hline Potassium & 0.21 & 5490 & & & - \\
\hline Selenium & & & 0.02 & 0.071 & - \\
\hline Silicon & 0.03 & 5020 & & & - \\
\hline Sodium & 10 & 204302 & & & - \\
\hline Strontium & 0.03 & 13100 & & & - \\
\hline Sulfate & 0 & 3663 & & 3000 & Yes \\
\hline Vanadium & & & & & - \\
\hline Zinc & 0 & 20 & 0.005 & 5 & Yes \\
\hline
\end{tabular}


Table 12. FAO irrigation criteria and shale gas produced water characteristics for identification of COCs. All units are $\mathrm{mg} / \mathrm{l}$ unless noted.

\begin{tabular}{lccccc}
\hline \multicolumn{1}{c}{ Constituents } & \multicolumn{2}{c}{ Shale Gas Produced } & Water & FAO Irrigation Criteria & COCs \\
\cline { 2 - 5 } Total Suspended Solids & Min & Max & Long & Short & \\
Total Dissolved Solids & 200 & 15000 & $<50^{\mathrm{a}}$ & $>100^{\mathrm{a}}$ & Yes \\
pH (S.U.) & 1724 & 228971 & $500^{\mathrm{b}}$ & $2000^{\mathrm{a}}$ & Yes \\
Aluminum & 1.21 & 8.36 & $6.0^{\mathrm{b}}$ & $>8.0^{\mathrm{a}}$ & Yes \\
Bicarbonate & 0 & 5290 & $5^{\mathrm{a}}$ & $20^{\mathrm{b}}$ & Yes \\
Boron & 0 & 4370 & $0^{\mathrm{a}}$ & $10^{\mathrm{a}}$ & Yes \\
Calcium & 0 & 10600 & $0.75^{\mathrm{b}}$ & $2^{\mathrm{a}}$ & Yes \\
Chloride & & & $0^{\mathrm{a}}$ & $20^{\mathrm{a}}$ & Yes \\
Copper & & $0^{\mathrm{a}}$ & $30^{\mathrm{a}}$ & Yes \\
Fluoride & 0 & $0.2^{\mathrm{a}}$ & $5^{\mathrm{b}}$ & Yes \\
Iron & 0.8 & 33 & $1^{\mathrm{a}}$ & $15^{\mathrm{b}}$ & Yes \\
Lithium & & 105 & $5^{\mathrm{a}}$ & $20^{\mathrm{b}}$ & Yes \\
Magnesium & 1.1 & $2.5^{\mathrm{a}}$ & $2.5^{\mathrm{b}}$ & Yes \\
Manganese & 0 & 25340 & $0^{\mathrm{a}}$ & $5^{\mathrm{a}}$ & Yes \\
Nitrate-N & & 96.5 & $0.2^{\mathrm{a}}$ & $10^{\mathrm{b}}$ & Yes \\
Phosphate-P & 0 & & $0^{\mathrm{a}}$ & $10^{\mathrm{a}}$ & Yes \\
Potassium & 0 & 2670 & $0^{\mathrm{a}}$ & $2^{\mathrm{a}}$ & Yes \\
Sodium & 0.3 & $0^{\mathrm{a}}$ & $2^{\mathrm{a}}$ & Yes \\
Sulfate & 10 & 5490 & $0^{\mathrm{a}}$ & $40^{\mathrm{a}}$ & Yes \\
Zinc & 0 & 204302 & $0^{\mathrm{a}}$ & $20^{\mathrm{a}}$ & Yes \\
\hline
\end{tabular}

a Ayers and Westcot, 1994

b USEPA, 2006

\section{Oil Field Produced Water Reuse}

COCs were identified by comparing concentrations in the OFPW with guideline concentrations for two water reuses (irrigation and livestock watering). These reuses were selected based on geographic location, climate, and economic need. If the concentration of any given parameter was greater than the concentration guideline for either irrigation or livestock, the parameter was considered a COC (Table 16).

\section{Conventional Natural Gas Produced Water Reuse}

Surface water discharge (i.e. surface water augmentation) was chosen as a potential reuse option for conventional NGPW based on location (e.g. latitude, longitude and altitude) and socioeconomic needs. The constituents of conventional NGPW were compared to USEPA NPDES surface water discharge criteria (Table 17). The COCs in conventional NGPW are: TSS, TDS, $\mathrm{Al}, \mathrm{Ba}, \mathrm{B}, \mathrm{Cd}, \mathrm{Cl}, \mathrm{Cu}, \mathrm{Fe}, \mathrm{Pb}, \mathrm{Mn}$, and Ni. Additional COCs, which are not specifically identified because of lack of reported analytical data, may include organic constituents such as oil and grease. 
Table 13. Identification of COCs in CBMPW for reuse in irrigation. (All units are $\mathrm{mg} / \mathrm{L}$ unless noted)

\begin{tabular}{|c|c|c|c|c|}
\hline Parameter & $\begin{array}{l}\text { Maximum Guideline } \\
\text { Concentration }\end{array}$ & $\begin{array}{l}\text { Maximum Levels } \\
\text { in CBMPW }\end{array}$ & $\begin{array}{l}\text { Mean Levels in } \\
\text { CBMPW }\end{array}$ & $\begin{array}{l}\text { Constituent of } \\
\text { Concern** }\end{array}$ \\
\hline Conductivity $(\mathrm{ms} / \mathrm{m})$ & $1700-13000$ & 145000 & 4419 & Yes \\
\hline pH (S. U.) & 4.5-9.0 & $6.56-9.86$ & - & Yes \\
\hline SAR (ratio) & 15 & 271 & 52.6 & Yes \\
\hline Bicarbonate & 520 & 1100 & 668 & Yes \\
\hline Carbonate & 3 & 59 & 24.4 & Yes \\
\hline Sulfate & 960 & 5590 & 335 & Yes \\
\hline Nitrate-N & 10 & 18.700 & 1.22 & Yes \\
\hline Ammonium-N & 5 & 59 & 3.53 & Yes \\
\hline Phosphate-P & 2 & 1.50 & 0.232 & No \\
\hline $\mathrm{Al}$ & 5 & 1.24 & 0.201 & No \\
\hline As & 0.1 & 0.060 & 0.003 & No \\
\hline B & $0.5-15$ & 2.40 & 0.151 & No \\
\hline $\mathrm{Be}$ & 0.1 & bdl & bdl & No \\
\hline $\mathrm{Ca}$ & 400 & 5870 & 53.6 & Yes \\
\hline $\mathrm{Cd}$ & 0.01 & 0.010 & 0.005 & Yes \\
\hline $\mathrm{Cl}$ & 1050 & 70100 & 845 & Yes \\
\hline Co & 0.05 & 0.0007 & 0.0002 & No \\
\hline $\mathrm{Cr}$ & 0.1 & 0.053 & 0.011 & No \\
\hline $\mathrm{Cu}$ & 0.2 & 0.060 & 0.009 & No \\
\hline $\mathrm{F}$ & 1 & 15.2 & 2.51 & Yes \\
\hline $\mathrm{Fe}$ & 5 & 220 & 2.73 & Yes \\
\hline K & 2 & 186 & 10.6 & Yes \\
\hline $\mathrm{Li}$ & 2.5 & 6.88 & 0.146 & Yes \\
\hline $\mathrm{Mg}$ & 60 & 1830 & 34.5 & Yes \\
\hline $\mathrm{Mn}$ & 0.2 & 5.40 & 0.059 & Yes \\
\hline Mo & 0.01 & 0.100 & 0.029 & Yes \\
\hline $\mathrm{Na}$ & 920 & 34100 & 1180 & Yes \\
\hline $\mathrm{Ni}$ & 0.2 & 0.203 & 0.020 & Yes \\
\hline $\mathrm{Pb}$ & 5 & 0.585 & 0.118 & No \\
\hline $\mathrm{Se}$ & 0.02 & 0.070 & 0.007 & Yes \\
\hline V & 0.1 & 0.059 & 0.013 & No \\
\hline $\mathrm{Zn}$ & 2 & 0.590 & 0.037 & No \\
\hline
\end{tabular}

(Ayers and Westcott, 1994; WDEQ, 2001; USEPA, 2004)

* Below detection limit (bdl)

** COCs are identified as constituents in CBMPW samples that have maximum concentrations in excess of the reuse guidelines; not all constituents identified as COCs in this study are of concern in all CBMPWs 
Table 14. Identification of COCs in CBMPW for reuse in livestock watering (All units are $\mathrm{mg} / \mathrm{L}$ unless noted)

\begin{tabular}{lcccc}
\hline Parameter & $\begin{array}{c}\text { Maximum } \\
\text { Guideline } \\
\text { Concentration }\end{array}$ & $\begin{array}{c}\text { Maximum Levels } \\
\text { in CBMPW }\end{array}$ & $\begin{array}{c}\text { Mean Levels in } \\
\text { CBMPW }\end{array}$ & $\begin{array}{c}\text { Constituent of } \\
\text { Concern }\end{array}$ \\
\hline Conductivity (ms/m) & 16000 & 145000 & 4420 & Yes \\
pH (S.U.) & $6.5-8.5$ & $6.56-9.87$ & - & Yes \\
Nitrate+Nitrite-N & 100 & 18.7 & 1.22 & No \\
Nitrite-N & 10 & bdl & bdl & No \\
$\mathrm{Al}$ & 5 & 1.24 & 0.201 & No \\
$\mathrm{As}$ & 0.2 & 0.060 & 0.003 & No \\
$\mathrm{B}$ & 5 & 2.40 & 0.151 & No \\
$\mathrm{Be}$ & 0.1 & bdl & bdl & No \\
$\mathrm{Cd}$ & 0.05 & 0.010 & 0.005 & No \\
$\mathrm{Co}$ & 1 & 0.001 & 0.000 & No \\
$\mathrm{Cr}$ & 1 & 0.053 & 0.011 & No \\
$\mathrm{Cu}$ & 0.5 & 0.060 & 0.009 & No \\
$\mathrm{F}$ & 2 & 15.2 & 2.52 & Yes \\
$\mathrm{Hg}$ & 0.01 & 0.0004 & 0.0002 & No \\
$\mathrm{Mn}$ & 0.05 & 5.40 & 0.059 & Yes \\
$\mathrm{Pb}$ & 0.1 & 0.585 & 0.118 & Yes \\
$\mathrm{Se}$ & 0.05 & 0.070 & 0.007 & Yes \\
$\mathrm{V}$ & 0.1 & 0.059 & 0.013 & No \\
$\mathrm{Zn}$ & 24 & 0.590 & 0.037 & No \\
\hline
\end{tabular}

*Below Detection Limit (bdl)

(Ayers and Westcot, 1994; WDEQ, 2001)

Table 15. Identification of COCs in CBMPW for reuse in aquaculture (All units are $\mathrm{mg} / \mathrm{L}$ unless noted)

\begin{tabular}{lcccc}
\hline Parameter* & $\begin{array}{c}\text { Maximum Guideline } \\
\text { Concentration }\end{array}$ & $\begin{array}{c}\text { Maximum Levels } \\
\text { in CBMPW }\end{array}$ & $\begin{array}{c}\text { Mean Levels in } \\
\text { CBMPW }\end{array}$ & $\begin{array}{c}\text { Constituent of } \\
\text { Concern }\end{array}$ \\
\hline $\mathrm{pH}$ (S.U.) & $6.5-9.5$ & $6.56-9.87$ & - & Yes \\
$\mathrm{Al}$ & 0.1 & 1.24 & 0.201 & Yes \\
$\mathrm{As}$ & 0.15 & 0.060 & 0.003 & No \\
$\mathrm{Ba}$ & 5 & 190 & 7.04 & Yes \\
$\mathrm{Cd}$ & 0.002 & 0.010 & 0.005 & Yes \\
$\mathrm{Cu}$ & 0.01 & 0.060 & 0.009 & Yes \\
$\mathrm{Fe}$ & 1 & 220 & 2.74 & Yes \\
$\mathrm{Mn}$ & 1.5 & 5.40 & 0.059 & Yes \\
$\mathrm{Pb}$ & 2.5 & 0.585 & 0.118 & No \\
$\mathrm{Se}$ & 0.005 & 0.070 & 0.007 & Yes \\
$\mathrm{Zn}$ & 0.1 & 0.590 & 0.037 & Yes \\
\hline
\end{tabular}

(WDEQ, 2001) 
Table 16. Comparison of oilfield produced water composition (maximum concentration) with water quality guidelines for irrigation and livestock watering. Constituents of concern identified by OFPW concentration exceeding guideline concentration. - indicates guideline concentration not listed in searchable database.

\begin{tabular}{|c|c|c|c|c|c|}
\hline \multirow[b]{2}{*}{ Parameter } & \multirow[b]{2}{*}{$\begin{array}{c}\text { OFPW Sample } \\
\text { Concentration }(\mathrm{mg} / \mathrm{L})\end{array}$} & \multicolumn{2}{|c|}{ Irrigation } & \multicolumn{2}{|c|}{ Livestock } \\
\hline & & $\begin{array}{c}\text { Guideline } \\
\text { Concentration }\end{array}$ & $\begin{array}{l}\text { Constituent } \\
\text { of Concern }\end{array}$ & $\begin{array}{c}\text { Guideline } \\
\text { Concentration }\end{array}$ & $\begin{array}{l}\text { Constituent } \\
\text { of Concern }\end{array}$ \\
\hline Alkalinity as $\mathrm{CaCO}_{3}$ & 380 & - & - & - & - \\
\hline Barium & 7.4 & - & - & - & - \\
\hline Bicarbonate & 976 & 610 & YES & - & - \\
\hline Calcium & 300 & 400 & YES & - & - \\
\hline Carbonate & 14.6 & 3 & - & - & - \\
\hline Chloride & 48 & 1050 & NO & - & - \\
\hline Iron (III) & 171 & - & - & - & - \\
\hline Iron (soluble) & 0.5 & 0.2 & YES & - & - \\
\hline Hardness as $\mathrm{CaCO}_{3}$ & 20 & - & - & - & - \\
\hline Magnesium & 8.7 & 60 & NO & - & - \\
\hline Manganese & 8.1 & 0.2 & YES & 0.05 & YES \\
\hline Nickel & 9.5 & 0.2 & YES & - & - \\
\hline Nitrate & 2 & 10 & NO & - & - \\
\hline Oil and grease & 92 & 35 & YES & 35 & YES \\
\hline Dissolved oxygen & 0.0002 & - & - & - & - \\
\hline Potassium & 42.6 & 2 & YES & - & - \\
\hline Silicon & 13.2 & - & - & - & - \\
\hline Sodium & 339 & 920 & NO & - & - \\
\hline Strontium & 2.2 & - & - & - & - \\
\hline Sulfate & 3 & 960 & NO & - & - \\
\hline TDS & 1370 & 500 & YES & - & - \\
\hline Tin & 1.3 & - & - & - & - \\
\hline Zinc & 17.4 & 2 & YES & 24 & NO \\
\hline
\end{tabular}


Table 17. Identification of COCs in conventional NGPW for surface water discharge. All units are mg/l unless noted.

\begin{tabular}{lccccc}
\hline \multirow{2}{*}{ Constituent } & \multicolumn{4}{c}{ Natural Gas PW } & \multicolumn{2}{c}{ NPDES } \\
(surface water) & COCs \\
\cline { 2 - 5 } Total Suspended Solids & Min & Max & Min & Max & \\
Total Dissolved Solids & 8 & 5484 & & 45 & Yes \\
pH & 2600 & 360000 & 30 & 5000 & Yes \\
Temperature ${ }^{\circ} \mathrm{C}$ & 3.1 & 7 & $>6$ & $>9$ & No \\
Aluminum & & & & $>40$ & No \\
Barium & bdl & 83 & & 0.05 & Yes \\
Boron & bdl & 1740 & & 154 & Yes \\
Cadmium & bdl & 56 & & 1 & Yes \\
Chloride & bdl & 1.21 & & 0.0025 & Yes \\
Chromium & 1400 & 190000 & & 5000 & Yes \\
Copper & bdl & 0.03 & 0.1 & 0.2 & No \\
Iron & bdl & 5 & 0.37 & 1 & Yes \\
Lead & bdl & 1100 & 0.6 & 9.4 & Yes \\
Manganese & $<0.2$ & 10.2 & 0.0056 & 0.015 & Yes \\
Nickel & 0.045 & 6.5 & 0.05 & 4.4 & Yes \\
Sulfate & bdl & 9.2 & 0.052 & 2 & Yes \\
Zinc & $<1.0$ & 47 & & 3000 & No \\
\hline
\end{tabular}

* Below Detection Limit (bdl)

Tight Gas Sand Produced Water Reuse

TGSPW, which is extracted in many states (e.g. New York, Ohio, West Virginia, Wyoming, and Colorado), has a variety of potential reuses, including irrigation and livestock. Reuse options were determined by location (e.g. latitude, longitude and altitude) and socio-economic needs. TGSPW as livestock water allows for a cost-effective alternative to municipal water. Irrigation may be a feasible reuse option where suitable water supply is limited because of drought or pollution (Frankenberger and Engberg 1998). Based on comparison of the constituents of TGSPW with USEPA FAO irrigation criteria (Table 18) the following were identified as COCs: TDS, pH, oil and grease, bicarbonate, $\mathrm{Ca}, \mathrm{Cl}, \mathrm{Fe}, \mathrm{K}, \mathrm{Mg}, \mathrm{Na}, \mathrm{Pb}$, and sulfate. The constituents of TGSPW were also compared to criteria for livestock and wildlife (Table 19), and the following were identified as COCs: $\mathrm{pH}$, oil and grease, $\mathrm{Cu}$, and $\mathrm{Mn}$.

External reuse options were chosen for these PWs because of their stringent requirements. Internal reuse options would be an alternative to selected external reuse options (e.g. surface water discharge) since internal reuse criteria are less stringent. 
Table 18. FAO irrigation criteria and tight gas sand produced water characteristics for identification of COCs. All units are $\mathrm{mg} / \mathrm{l}$ unless noted.

\begin{tabular}{|c|c|c|c|c|c|}
\hline \multirow[b]{2}{*}{ Constituents } & \multicolumn{2}{|c|}{$\begin{array}{c}\text { Tight Gas Sand Produced } \\
\text { Water }\end{array}$} & \multicolumn{2}{|c|}{$\begin{array}{c}\text { FAO Irrigation } \\
\text { Criteria }\end{array}$} & \multirow[b]{2}{*}{$\mathrm{COCs}$} \\
\hline & Min & Max & Min & $\operatorname{Max}$ & \\
\hline Total Suspended Solids & & 85.7 & $<50^{\mathrm{a}}$ & $>100^{\mathrm{a}}$ & No \\
\hline Total Dissolved Solids & 1588 & 343300 & $500^{\mathrm{b}}$ & $2000^{\mathrm{a}}$ & Yes \\
\hline pH (S.U.) & 5 & 8.6 & $6.0^{\mathrm{b}}$ & $>8.0^{\mathrm{a}}$ & Yes \\
\hline Conductivity & & 24.4 & 1700 & 13000 & No \\
\hline Oil and Grease & & 42 & & 35 & Yes \\
\hline Ammonium-N & & 2.735 & $0^{\mathrm{a}}$ & $5^{\mathrm{a}}$ & No \\
\hline Arsenic & & 0.17 & $0.1^{\mathrm{a}}$ & 2 & No \\
\hline Bicarbonate & 854 & 1147 & $0^{\mathrm{a}}$ & $10^{\mathrm{a}}$ & Yes \\
\hline Cadmium & & 0.037 & $0.01^{\mathrm{a}}$ & $0.05^{\mathrm{b}}$ & No \\
\hline Calcium & 3 & 74185 & $0^{\mathrm{a}}$ & $20^{\mathrm{a}}$ & Yes \\
\hline Chloride & 52 & 216000 & $0^{\mathrm{a}}$ & $30^{\mathrm{a}}$ & Yes \\
\hline Chromium & & 0.265 & $0.1^{\mathrm{a}}$ & $1^{\mathrm{b}}$ & No \\
\hline Copper & & 0.539 & $0.2^{\mathrm{a}}$ & $5^{\mathrm{b}}$ & No \\
\hline Iron & & 0.015 & $5^{\mathrm{a}}$ & $20^{\mathrm{b}}$ & Yes \\
\hline Lead & & 0.488 & $5^{\mathrm{a}}$ & $10^{\mathrm{b}}$ & Yes \\
\hline Magnesium & 2 & 8750 & $0^{\mathrm{a}}$ & $5^{\mathrm{a}}$ & Yes \\
\hline Manganese & & 0.525 & $0.2^{\mathrm{a}}$ & $10^{\mathrm{b}}$ & No \\
\hline Nickel & & 0.123 & $0.2^{\mathrm{a}}$ & $2^{\mathrm{b}}$ & No \\
\hline Potassium & 5 & 2500 & $0^{\mathrm{a}}$ & $2^{\mathrm{a}}$ & Yes \\
\hline Sodium & 648 & 6273 & $0^{\mathrm{a}}$ & $40^{\mathrm{a}}$ & Yes \\
\hline Sulfate & 12 & 48 & $0^{\mathrm{a}}$ & $20^{\mathrm{a}}$ & Yes \\
\hline Zinc & & 0.076 & $2^{\mathrm{a}}$ & $10^{\mathrm{b}}$ & Yes \\
\hline
\end{tabular}

Table 19. Livestock and Wildlife criteria and tight gas sand produced water characteristics for identification of COCs. All units are $\mathrm{mg} / \mathrm{l}$ unless noted.

\begin{tabular}{cccccc}
\hline & & & \multicolumn{2}{c}{ Livestock and } \\
& Tight Gas Produced Water & Wildlife \\
\hline Constituents & Min & Max & Min & Max & COCs \\
pH & 5 & 8.6 & 6.5 & 8.5 & Yes \\
Conductivity & & 24.4 & & 16000 & No \\
Oil and Grease & & 42 & 35 & Yes \\
Arsenic & 0.17 & 0.2 & No \\
Cadmium & 0.037 & 0.05 & No \\
Chromium & 0.265 & 1 & No \\
Copper & 0.539 & 0.5 & Yes \\
Lead & & 0.488 & 5 & No \\
Manganese & & 0.525 & & 0.05 & Yes \\
Zinc & & 0.076 & & 2 & No \\
\hline
\end{tabular}


Design and Construct Pilot-Scale Constructed Wetland Treatment Systems (Task 4)

The objective of Task 4 is to design and construct pilot-scale constructed wetland treatment systems (CWTSs) targeting specific COCs in various produced water types to meet reuse criteria. Prior to designing specific treatment systems, produced waters were characterized (Task 2 ), and these characteristics were compared to specific reuse criteria (Task 3).

Wetlands offer natural environments within which unique reactions can occur (Rodgers and Castle 2008). CWTSs can be designed in a way that allows these specific reactions to take place for treatment (i.e. transfers and transformations) of targeted constituents. The benefits of CWTSs can include: low cost of operation, low maintenance, effective treatment, solar energy driven, increased effectiveness over time, tolerance of deviations in flow rate and contaminant load, and treatment of multiple COCs simultaneously and more effectively than chemical or physical treatment processes (Bhamidimarri et al. 1999; Sundaravadivel and Vigneswaran 2001; Rodgers and Castle 2008; Dorman et al. 2010). In conjunction with other treatment methods (e.g. oilwater separators, reverse osmosis and granular activated carbon (GAC) systems), hybrid CWTSs can provide effective water treatment, provided that COCs are successfully targeted through operative pathways (Murray Gulde et al. 2005a; Johnson et al. 2008b). CWTSs can be specifically designed based on targeted constituents and/or treatment pathways to transfer or transform those constituents (Hawkins et al. 1997; Gillespie et al. 1999, 2000).

CWTSs offer a potential option to conventional treatment techniques for mitigation of risks from produced water. With regard to CWTSs, there are essentially two types of produced water (PW): fresh PW and PW with elevated salinity. A CWTS designed to receive fresh PW can be designed based on COCs in a specific PW; however, in higher salinity PWs, hybrid CWTSs (i.e. CWTS with reverse osmosis) are often needed to pre-treat the PW prior to entry into a CWTS. Using COCs identified through the initial produced water characterization, CWTSs were specifically designed to transfer and transform constituents in produced water in order to decrease concentrations of targeted COCs. Pilot-scale CWTSs, while sufficiently small to enable control and manipulation of macrofeatures (e.g. hydroperiod, hydrosoil, and vegetation) and allow replication (Hawkins et al. 1997), are sufficiently large to predict performance of a full scale CWTS in terms of COC removal rates and extents (Rodgers and Castle 2008; Dorman et al. 2010).

The design of a CWTS for a specific water (e.g. produced water) includes consideration of the demographic, geologic, and meteorological characteristics of the area from which a PW originates. Depending on these conditions, a CWTS can be designed in one of two ways: subsurface flow (SSF) or free-water surface (FWS) (Figure 1). SSF CWTSs can be further subdivided into vertical flow and horizontal flow designs. Both vertical and horizontal flow SSF CWTSs are designed in a manner that maintains water levels below the top of the aggregate (e.g. rock, gravel); this allows for reduced exposure to elements at the surface (e.g. humans, wildlife, sunlight, wind, etc.) (Halverson 2004). FWS CWTSs have been used for treatment of many waters, including energy derived waters, containing many different COCs (Knight et al. 1999; Gillespie et al. 2000; Eggert et al. 2008; Johnson et al. 2008b); FWS systems are generally larger than SSF systems, but usually cost less to construct and operate/maintain (Halverson 2004). Both SSF and FWS typically exhibit lower capital costs, as well as operational/maintenance costs, than conventional water treatment systems (Halverson 2004; Mooney and Murray Gulde 2008). 
Pilot scale CWTSs offer the ability to experimentally test hypotheses under a variety of conditions prior to investing in full scale CWTSs (Rodgers and Castle, 2008). Factors such as treatment rates and extents may be evaluated to determine critical full scale design parameters (e.g. loading rate, wetland area, and hydraulic retention time). In addition, the use of pilot scale CWTSs may reduce full scale performance uncertainties.

Since PWs may contain high levels of dissolved solids, low molecular weight organics, oil and grease, metals, metalloids, and ammonia, four pilot-scale CWTSs were designed and constructed. The pilot-scale CWTSs include: (1) a post-reverse osmosis (RO) PW treating system, (2) a metalloid (e.g. Se, As) and low molecular weight organic (LMWO) treating system, (3) a divalent metal (e.g. $\mathrm{Zn}, \mathrm{Cu}, \mathrm{Cd}, \mathrm{Ni}$ ) and LMWO treating system, (4) and an oil \& grease and metal (e.g. Ni, Zn, Fe, Mn) treating system. Specific treatment pathways for each system were identified by reviewing peer-reviewed publications including Eh-pH diagrams (Brookins 1988), previous CWTS studies, and reduction-oxidation reactions. Each system was then specifically designed and constructed to promote the conditions required that enable the treatment pathways. Specific treatment pathways include nitrification-denitrification, reductionoxidation, biodegradation, precipitation, and sorption.

\section{Pilot-Scale CWTS for Simulated Post-RO Produced Water}

In waters containing elevated levels of dissolved chlorides, which may adversely impact wetland biota, an external treatment source may be required. Reverse osmosis offers the ability to remove many dissolved solids from produced waters (Veil et al, 2004). However, constituents may pass through the RO membranes. In San Ardo, California, RO is used to treat produced water containing dissolved solids and boron (Myers, pers. comm., 2008). The effluent of the treated water still contains levels of ammonia approaching $20 \mathrm{mg} / \mathrm{L}$ (Myers, pers. comm.). Other studies have found ammonia to be a major concern in post-RO treated water (Liske, 2005). For these reasons, ammonia is an important COC in post-RO treated produced water; therefore, a pilot scale CWTS was designed and constructed to treat ammonia in simulated post-RO water.

To assess the removal of ammonia by CWTSs, a FWS pilot scale system was designed and constructed to target the transformation of ammonia to nitrogenous gas by a three step process: (1) nitrification of ammonia to nitrite, (2) nitrification of nitrite to nitrate, (3) and reduction of nitrate to nitrogen gas. Because nitrification and denitrification involve different geochemical conditions (Table 20), special design considerations were made to allow microbial transformations to operate sequentially through the system.

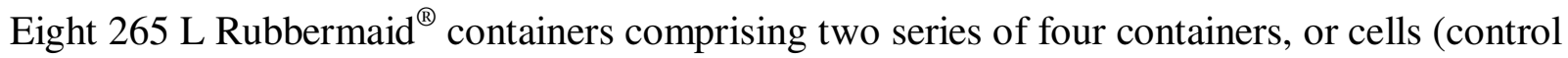
and experimental), were filled with approximately $15 \mathrm{~cm}$ of fluvial sediment collected near Eighteen Mile Creek located near Clemson University. Connections between cells were built using poly-vinyl chloride (PVC) pipes with adjustable overflow levels to control water depth and allow gravity flow from each cell. Each cell was planted with approximately 20 broadleaf cattails (Typha latifolia) collected from a nearby aquaculture pond. The first cell from each of the two series is connected to a $5678 \mathrm{~L}$ polypropylene carboy holding tank containing simulated post-RO PW (20 mg/L ammonia) by a Fluid Metering, Inc. ${ }^{\circledR}$ (FMI ${ }^{\circledR}$ ) piston pump. The pump delivers 90 
$\mathrm{mL} / \mathrm{minute}$ to one series for a hydraulic retention time of 24 hours per cell (control) and 45 $\mathrm{mL} / \mathrm{minute}$ to the other series for a hydraulic retention time (HRT) of 48 hours per cell (experimental). Aeration is supplied to the first cell of the experimental series by a submerged, slotted PVC pipe connected to an air pump. Organic carbon is supplied to the second cell of the experimental series using an $\mathrm{FMI}^{\circledR}$ pump delivering $0.88 \mathrm{~mL} / \mathrm{minute}$ of a $20 \mathrm{mg} / \mathrm{mL}$ solution of sucrose. A schematic of the design is shown in Figure 2.

In the pilot scale CWTS, the first process in ammonia removal is oxidation of ammonia to nitrite by autotrophic nitrifying bacteria, predominately Nitrosomonas (Watson et al. 1981), as shown in equation 1.

$\mathrm{NH}_{3}+\mathrm{O}_{2}>\mathrm{NO}_{2}^{-}+3 \mathrm{H}^{+}+2 \mathrm{e}^{-} \quad$ Eqn. 1 (Gambrell and Patrick, 1978)

Once oxidized to nitrite, further oxidization by autotrophic nitrifying bacteria, predominantly Nitrobacter (Watson et al. 1981), results in the formation of nitrate as (equation 2).

$\mathrm{NO}_{2}^{-}+\mathrm{H}_{2} \mathrm{O}>\mathrm{NO}_{3}^{-2}+2 \mathrm{H}^{+}+2 \mathrm{e}^{-} \quad$ Eqn. 2 (Gambrell and Patrick, 1978)

The final process in ammonia removal is the reduction of nitrate by heterotrophic facultative anaerobes (equation 3).

$6 \mathrm{NO}_{3}{ }^{-2}+5 \mathrm{CH}_{3} \mathrm{OH}>5 \mathrm{CO}_{2}+3 \mathrm{~N}_{2}+7 \mathrm{H}_{2} \mathrm{O}+6 \mathrm{OH}^{-} \quad$ Eqn. 3 (Knowles, 1981)

For nitrification, a high level of dissolved oxygen $(>2.0 \mathrm{mg} / \mathrm{L})$ is required in addition to a near neutral pH and sufficient levels of alkalinity (>100 mg/L) (Odell et al. 1996; USEPA 2002b). To help meet the high dissolved oxygen demand, broadleaf cattails ( $T$. latifolia) were selected to increase soil aeration and consequently increase dissolved oxygen levels in the water column. In addition, a FWS design was selected to enhance diffusion of oxygen into the water by maximizing the surface area of the water/atmosphere interface. To ensure proper $\mathrm{pH}$ throughout the nitrification process, crushed oyster shells ( $50 \mathrm{~g}$ per cell) were added to the hydrosoil. The ground oyster shells ( $98 \% \mathrm{CaCO}_{3}$ by weight) act as both a $\mathrm{pH}$ buffer and a source of alkalinity for nitrification; nitrification requires $8.64 \mathrm{mg} / \mathrm{L}$ of bicarbonate per $\mathrm{mg} / \mathrm{L}$ of ammonia removed (Gujer and Jenkins 1974). To further test the ability of amendments to enhance nitrification, an external source of aeration is applied to the first cell of the experimental series.

For denitrification, a low level of dissolved oxygen $(<2.0 \mathrm{mg} / \mathrm{L})$ is required in addition to a circumneutral $\mathrm{pH}$. There must also be available dissolved organic carbon as an energy and electron source for the heterotrophic facultative anaerobes. To meet these criteria, the system is designed to allow detritus to serve as a cumulative source of dissolved organic material throughout each of the four cells in each series. This accumulation of dissolved organic carbon decreases the dissolved oxygen level through heterotrophic decomposition and reduces the oxidation/reduction potential (Eh) by adding reduced organic carbon to the hydrosoil. As with the nitrification steps, ground oyster shells were added to the denitrifying cells to maintain neutral $\mathrm{pH}$ and alkalinity. To further test the ability of amendments to enhance denitrification, an external source of carbon (sucrose) is connected to the second cell of the experimental series. 
To address nutrient demands presented by the macrophytes and microbes, a time-release, nitrogen free fertilizer (Osmocote ${ }^{\circledR}$ ) is added to the hydrosoil. Selection of an appropriate fertilizer is crucial as many commercially sold fertilizers may contain additional ammonia or nitrate.

\section{Pilot-Scale CWTS for PW Containing Divalent Metals and Low Molecular Weight Organics}

The pilot-scale CWTS is specifically designed to target or enhance pathways of removal (i.e. transfers or transformations) of divalent metals $\left(\mathrm{Cd}^{+2}, \mathrm{Cu}^{+2}, \mathrm{Ni}^{+2}\right.$, and $\left.\mathrm{Zn}^{+2}\right)$ and low molecular weight organics (LMWO) (e.g. BTEX) in simulated PW. Divalent metals may be removed under specific operating conditions (Table 21) through biogeochemical processes including: precipitation through dissimilatory sulfate reduction, organic complexation with detritus, hydrolysis, and sorption to biotic and abiotic exchange sites (Hawkins et al. 1997; Ford 1998; Murray-Gulde et al. 2005a; Rodgers and Castle 2008). Organic constituents may be removed under specific operating conditions (Table 21) through processes including: biodegradation, sorption, photolysis, and volatilization (Hawkins et al. 1997; Murray-Gulde et al. 2003; Johnson et al. 2008b; Rodgers and Castle 2008). The primary targeted pathway for removal of divalent metals (e.g. $\mathrm{Cd}^{+2}, \mathrm{Cu}^{+2}, \mathrm{Ni}^{+2}$, and $\mathrm{Zn}^{+2}$ ) is precipitation through dissimilatory sulfate reduction. In a reducing environment $(-100$ to $-350 \mathrm{mV})$, with adequate concentrations of sulfate and divalent metals, complexes may form between the divalent metals and sulfide (e.g. ZnS) through dissimilatory sulfate reduction (Allen et al. 1993). Hydrosoil redox ranging from -50 to $-250 \mathrm{mV}$ in reducing wetland reactors and circumneutral aqueous $\mathrm{pH}$ (6-9 S.U.) are favorable for dissimilatory sulfate reduction, which provides reduced sulfur for precipitating available cationic metals such as mercury, copper, lead, zinc, cadmium, and nickel (Brookins 1988; Morse 1993; Carbonell et al. 1999; Rodgers and Castle 2008). Metal-sulfide minerals are stable in aqueous systems and precipitate into the hydrosoil, significantly limiting metal bioavailability (Morse 1993). The pathway targeted for removal of LMWOs is biodegradation by microbes in an anaerobic environment (Lovley 1997). Findings that benzene can be rapidly degraded under sulfate-reducing conditions have enhanced the potential benefit of anaerobic metabolism to BTEX remediation (Edwards et al. 1992; Lovley et al. 1995; Lovley 1997).

In this pilot-scale system, a $3780 \mathrm{~L}$ polypropylene carboy retention basin is used for formulation and storage of simulated fresh PW. Simulated PW is homogenized using a $1 / 2 \mathrm{hp}$ mixing pump. Simulated PW from the retention basin is pumped using FMI ${ }^{\circledR}$ pumps into the first of the wetland cells. The wetland cells consist of two replicated series, each containing four wetland cells. Each

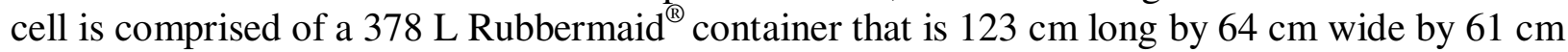
deep. The cells are connected by PVC pipe fittings approximately $6 \mathrm{~cm}$ below the top of each Rubbermaid $^{\circledR}$ container to allow gravity flow from each cell (Figure 3). Vegetation in the first wetland cell is giant bulrush (Schoenoplectus californicus C.A. Meyer). Schoenoplectus californicus was selected based on its ability to maintain reducing hydrosoil necessary for dissimilatory sulfate reduction (Josselyn et al. 1990; Sinicrope et al. 1992; Hawkins et al. 1997; Gillespie et al. 1999, 2000; Murray-Gulde et al. 2005b) and provide a source of organic carbon (detritus) over time for an energy source for sulfate reducing microbes (Moshiri 1993; Huddleston et al. 2000; Murray-Gulde et al. 2005b). Schoenoplectus californicus may also provide sorption sites for LMWOs (Brix 1994). The selected hydrosoil for the first two cells was river sediment (Eighteen Mile Creek, South Carolina) added to a depth of $30 \mathrm{~cm}$ to provide an 
adequate root zone for $S$. californicus. Amendments to the top $10 \mathrm{~cm}$ of the hydrosoil included $3 \%$ hay (by volume) as an organic matter and energy source and $1.5 \%$ pelletized gypsum $\left(\mathrm{CaSO}_{4} \cdot 2 \mathrm{H}_{2} \mathrm{O}\right)$ as a sulfur source for dissimilatory sulfate reduction (Vymazal et al. 1998). The vegetation for the final two cells in the series is narrowleaf cattail (Typha angustifolia L.). These final two cells contain porous quartz sand hydrosoil with no organic carbon amendments in order to facilitate aerobic conditions in order to increase the dissolved oxygen content for discharge (EPA 2005). Each cell contained approximately $140 \mathrm{~L}$ of surface water. By maintaining a flow rate of $97 \mathrm{~mL} / \mathrm{min}$, the nominal HRT of the pilot-scale CWTS is 24 hours per cell or 96 hours for the four wetland cells in series. The pilot-scale CWTS is constructed outdoors; an outdoor pilotscale CWTS will provide a representation of environmental conditions that may affect full-scale CWTSs.

\section{Pilot-Scale CWTS for PW Containing Metalloids and Low Molecular Weight Organics}

The pilot-scale experiment was designed based on results of Tasks 2 and 3, literature review regarding treatment pathways, and results from previous experiments (Eggert et al. 2008). The targeted pathways for removal of COCs in the pilot-scale CWTS may be contingent on maintenance of specific operating conditions (Table 22); therefore, regular monitoring of conditions is conducted. Removal of the metalloid elements As and Se may be achieved through precipitation; As may be coprecipitated with Fe (Edwards 1994; Hering et al. 1996), and Se may be precipitated through microbial reduction (Maiers et al. 1988; Lortie et al. 1992; Tomei et al. 1995; Garbisu et al. 1996; Ike et al. 2000; Zhang and Frankenberger 2005; Siddique et al. 2007; Zhang et al. 2008). Low molecular weight organic constituents may be removed through processes including biodegradation, sorption, photolysis, and volatilization (Hawkins et al. 1997; Johnson et al. 2008b; Rodgers and Castle 2008). The targeted removal pathway for As is coprecipitation with $\mathrm{Fe}$ and the targeted removal pathway for Se is microbial reduction in anaerobic conditions using microbial assemblages capable of dissimilatory selenium reduction. This process can transform aqueous As and Se into insoluble species that are unavailable for biological uptake, thus decreasing pilot-scale CWTS effluent concentrations of As and Se. The targeted pathway for removal of LMWOs is anaerobic microbial biodegradation.

A literature review provided evidence regarding an efficient pathway for Se treatment involving addition of an organic carbon source to enhance removal of soluble Se (selenate and selenite) from water through microbial reduction (Zawislanski et al. 2001b; Zhang and Frankenberger 2005) in anaerobic aquatic environments (Maiers et al. 1988; Siddique et al. 2007). This was incorporated into design of the pilot-scale CWTS by evaluating the efficiency of two different organic carbon/energy source amendments to enhance microbial abundance and activity: sucrose and a dried yeast product/nutrient additive (Aqua-Smart ${ }^{\mathrm{TM}}$, Diamond V Corporation, Cedar Rapids, IA). Therefore, two experimental treatment series will be evaluated (e.g. sucrose and dried yeast product) and compared to one untreated control series.

The pilot-scale CWTS uses a 5678 L polypropylene carboy retention basin to house simulated PW and twelve $378 \mathrm{~L}^{\text {Rubbermaid }}{ }^{\circledR}$ containers (123 cm long by $64 \mathrm{~cm}$ wide by $61 \mathrm{~cm}$ deep) placed into three series of four cells using a free-water surface (FWS) design (Figure 4). The cells are connected with PVC pipe fittings approximately $6 \mathrm{~cm}$ below the top of each Rubbermaid $^{\circledR}$ container to allow gravity flow from each cell. The untreated control series is 
designated series $\mathrm{C}$; the dried yeast culture treatment series is designated series $\mathrm{Y}$; the sucrose treatment series is designated series $\mathrm{S}$. Each cell is filled to a depth of approximately $30 \mathrm{~cm}$ with river sand from 18-mile Creek in Clemson, SC, and subsequently planted with T. latifolia harvested from an aquaculture pond on the Clemson University campus. The cells are planted at a density of approximately 25-30 plants per cell. Three piston pumps (FMI ${ }^{\circledR}$ QG400) deliver 128 $\mathrm{mL}$ simulated PW/minute to achieve a nominal 24-hr HRT per cell, or 96-hr per series. To maintain a circumneutral $\mathrm{pH}(6.5-8)$ and increase alkalinity in the pilot-scale CWTS, $1000 \mathrm{~g}$ of ground oyster shells $\left(98 \% \mathrm{CaCO}_{3}\right.$ by weight) were added to each treatment cell. To maintain reducing conditions, $100 \mathrm{~g}$ of zero-valent iron $\left(\mathrm{Fe}^{0}\right)$ were added to each treatment cell, as well as $12 \mathrm{~g}$ of 19-6-12 Osmocote ${ }^{\circledR}$ fertilizer to increase the nitrogen, phosphorus, and potassium concentrations to provide essential nutrients for the microbes and plants.

\section{Pilot-Scale CWTS for PW Containing Oil \& Grease and Divalent Metals}

A CWTS must be designed in a strategic way to facilitate biogeochemical reactions that will target specified COCs. Based on comparison of characteristics of the produced waters studied in Task 2 to water quality guidelines (Task 3), the following were identified as COCs in waters such as OFPW containing oil and grease: oil and grease, Fe, Mn, Ni, and Zn. The following biogeochemical pathways were identified for treating these COCs: dissimilatory sulfate reduction (metals), oxidation (oil and grease, metals), and sorption (oil and grease, metals) (Table 23). As oil and grease in the produced water enters the system for treatment, the oil and grease are expected to sorb to available sites (e.g. hydrosoil and plant roots). The upstream cells are expected to enter a reductive state in response to the oil and grease, thus enabling dissimilatory sulfate reduction to produce sulfides that may combine with cations (e.g. $\mathrm{Zn}$ and $\mathrm{Ni}$ ) to precipitate metal-sulfide minerals. The pilot-scale CWTS is designed for oil and grease content to decrease as the water moves through the system, resulting in oxidizing conditions in downstream cells. Remaining metals (e.g. Mn and Fe) will be transferred to the solid phase under oxidizing conditions.

A pilot-scale CWTS was constructed within a greenhouse owned by Clemson University. The pilot-scale system uses a $3785 \mathrm{~L}$ polypropylene carboy retention basin and three series of cells (Figure 5). Each series comprises four 378 L wetland cells (Rubbermaid ${ }^{\circledR}$ containers). Surface area of each wetland cell is $0.66 \mathrm{~m}^{2}$, and axial dimensions are $61 \mathrm{~cm}$ in height, $123 \mathrm{~cm}$ in length, and $64 \mathrm{~cm}$ in width at its widest point. Water residing in the retention basin is pumped continuously through $0.64 \mathrm{~cm}$ diameter plastic tubing to each of the three series using FMI ${ }^{\circledR}$ piston pumps. For each series, four cells are arranged at decreasing elevations to achieve gravity flow through the system. Water is transferred between the cells through $2.5 \mathrm{~cm}$ diameter PVC pipes. Two series incorporate a SSF design, and the third series incorporates a FWS design. The purpose of using two SSF series is for replication. The reasons for using a FWS series are: (1) for comparison of data with previous pilot-scale studies in which FWS cells were used and (2) for comparison of treatment effectiveness with the SSF series. In both the SSF and FWS series, the specific types of hydrosoil and plants selected promote oxidizing conditions (Armstrong et al. 1999; Kanagy et al. 2008a). Wetland cells for SSF series consist of a two layer hydrosoil of pea gravel and medium-sized gravel, and are planted with Phragmites australis to provide sorption sites and support oxidation. Wetland cells for FWS series consist of a hydrosoil of coarse, well sorted, quartz sand and are planted with Typha latifolia to promote oxidizing conditions in the 
hydrosoil. The wetland cells were amended with Osmocote ${ }^{\circledR}$ time-released fertilizer to provide nutrients required for plant growth, which are not found in sufficient quantities in the water supply or newly emplaced hydrosoil. Hydraulic retention time in each series is controlled by manipulating inflow rate.

Table 20. Nitrification and Denitrification Operating Conditions for Simulated Post-RO Produced Water Containing Ammonia in a Pilot-Scale Constructed Wetland Treatment System

\begin{tabular}{lllllllll}
\hline Constituent & Pathway & $\begin{array}{l}\text { Inflow } \\
\text { Conc. }\end{array}$ & $\begin{array}{l}\mathrm{pH} \\
(\mathrm{S} . \mathrm{U} .)\end{array}$ & $\begin{array}{l}\text { Temp } \\
\left({ }^{\circ} \mathrm{C}\right)\end{array}$ & Redox $(\mathrm{mV})$ & $\begin{array}{l}\text { Dissolved } \\
\text { Oxygen } \\
(\mathrm{mg} / \mathrm{L})\end{array}$ & $\begin{array}{l}\text { Alkalinity } \\
(\mathrm{mg} \\
\mathrm{CaCO} / \mathrm{L})\end{array}$ & $\begin{array}{l}\text { Organic } \\
\text { Carbon }\end{array}$ \\
\hline Ammonia & Nitrification & $20 \mathrm{mg} / \mathrm{L}$ & $6.6-9.7^{5}$ & $8-30^{7}$ & $(+100)-(+350)^{2}$ & $>2.0^{1}$ & $>100^{3}$ & $\begin{array}{l}\text { None } \\
\text { Nitrate }\end{array}$ \\
& Denitrification & None & $7.0-8.5^{1}$ & $>25^{6}$ & $(-50)-(+50)^{2}$ & $<0.5^{1}$ & $>100^{3}$ & $\begin{array}{l}\text { Organic } \\
\text { matter }\end{array}$ \\
\hline
\end{tabular}

(1) Gambrell and Patrick 1978; (2) Gerardi 2007; (3) Gujer and Jenkins 1974; (4) Knowles, R. 1981; (5) Odell et al. 1996; (6) Pierzynski et al. 2005; (7) USEPA 2002a

Table 21. Operating Conditions for Simulated PW Containing Divalent Metals and LMWOs in a Pilot-Scale Constructed Wetland Treatment System

\begin{tabular}{|c|c|c|c|c|}
\hline Constituents & $\begin{array}{l}\text { Concentration } \\
(\mathrm{mg} / \mathrm{L})\end{array}$ & Pathway & $\begin{array}{l}\mathrm{pH} \\
\text { (S.U.) }\end{array}$ & Redox \\
\hline Cadmium & 6.0 & $\begin{array}{l}\text { Sulfide formation; precipitation through } \\
\text { Dissimilatory Sulfate reduction }\end{array}$ & $6-9$ & Reducing -100 to $-250 \mathrm{mV}$ \\
\hline Copper & 4.0 & $\begin{array}{l}\text { Sulfide formation; precipitation through } \\
\text { Dissimilatory Sulfate reduction }\end{array}$ & $6-9$ & Reducing -100 to $-250 \mathrm{mV}$ \\
\hline Nickel & 6.0 & $\begin{array}{l}\text { Sulfide formation; precipitation through } \\
\text { Dissimilatory Sulfate reduction }\end{array}$ & $6-9$ & Reducing -100 to $-250 \mathrm{mV}$ \\
\hline Zinc & 6.0 & $\begin{array}{l}\text { Sulfide formation; precipitation through } \\
\text { Dissimilatory Sulfate reduction }\end{array}$ & $7-9$ & Reducing -100 to $-250 \mathrm{mV}$ \\
\hline LMWOs & 25.0 & $\begin{array}{l}\text { Biodegradation }{ }^{2} ; \text { Sorption; } \\
\text { Volatilization }\end{array}$ & & Reducing \\
\hline
\end{tabular}

(1) Brookins 1988; (2) Junfeng et al. 2007

Table 22. Operating Conditions for Simulated Produced Water Containing Metalloids and Low Molecular Weight Organics in a Pilot-Scale Constructed Wetland Treatment System

\begin{tabular}{|c|c|c|c|c|c|c|c|}
\hline Constituent & $\begin{array}{l}\text { Conc. } \\
(\mathrm{mg} / \mathrm{L})\end{array}$ & Pathway & pH (S.U.) & $\begin{array}{l}\text { Redox } \\
(\mathrm{mV})\end{array}$ & $\begin{array}{c}\mathrm{DO}(\mathrm{mg} \\
\left.\mathrm{O}_{2} / \mathrm{L}\right)\end{array}$ & $\begin{array}{l}\text { Alkalinity } \\
\left(\mathrm{mg}^{2}\right. \\
\left.\mathrm{CaCO}_{3} / \mathrm{L}\right)\end{array}$ & $\begin{array}{c}\text { Hardness } \\
(\mathrm{mg} \\
\left.\mathrm{CaCO}_{3} / \mathrm{L}\right)\end{array}$ \\
\hline Selenium & 0.050 & Microbial reduction 5 & $6.5-9.5^{1}$ & $<+50 \mathrm{mV}^{2}$ & $<3.0$ & $>50$ & $>50$ \\
\hline Arsenic & 0.020 & Coprecipitation $^{6}$ & $6.5-8$ & $<-200 \mathrm{mV}^{2}$ & $<2.0$ & $>50$ & $>50$ \\
\hline LMWOs & 25.0 & $\begin{array}{l}\text { Biodegradation }{ }^{3}, \\
\text { Sorption }\end{array}$ & $6.5-8$ & Reducing $^{4}$ & $>5.0$ & $>50$ & $>50$ \\
\hline
\end{tabular}

(1) Lortie et al. 1992; (2) Brookins 1988; (3) Junfeng et al. 2007; (4) Lovley 1997; (5) Zhang et al. 2008; (6) Edwards 1994 
Table 23. Operating conditions for simulated PW containing divalent metals and oil and grease

\begin{tabular}{lllll}
\hline Constituent & Pathway & Inflow conc. & pH $($ S.U. $)$ & Redox $(\mathrm{mV})$ \\
\hline Oil and Grease & Oxidation, Sorption & $25-100 \mathrm{mg} / \mathrm{L}$ & $6.5-8.0^{1}$ & $>-50^{1}$ \\
Zn, Ni & Sulfate Reduction, Sorption & $\mathrm{Zn}: 5 \mathrm{mg} / \mathrm{L}$ & $6.0-9.0^{1}$ & $<-150^{1}$ \\
& & $\mathrm{Ni}: 0.37 \mathrm{mg} / \mathrm{L}$ & & \\
$\mathrm{Fe}$ & Oxidation & $0.4 \mathrm{mg} / \mathrm{L}$ & $6.5-8.0^{1}$ & $>-50^{1}$ \\
$\mathrm{Mn}$ & Oxidation, Sorption & $0.5 \mathrm{mg} / \mathrm{L}$ & $6.5-8.0^{1}$ & $>-50^{1}$
\end{tabular}

(1) Brookins, 1988
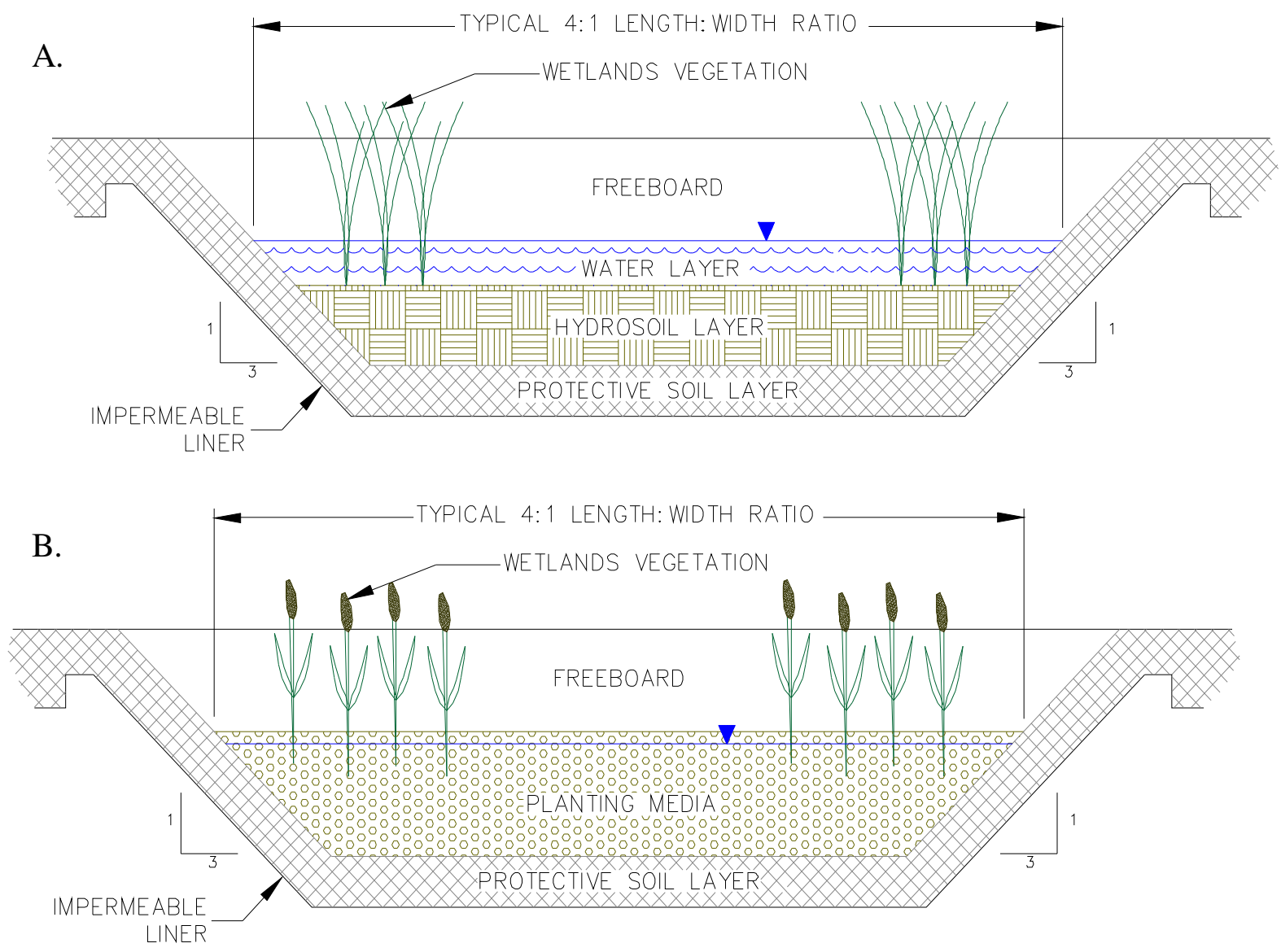

Figure 1 Schematic diagram for free-water surface (A) and sub-surface flow (B) CWTSs (Rodgers and Castle, 2008) 


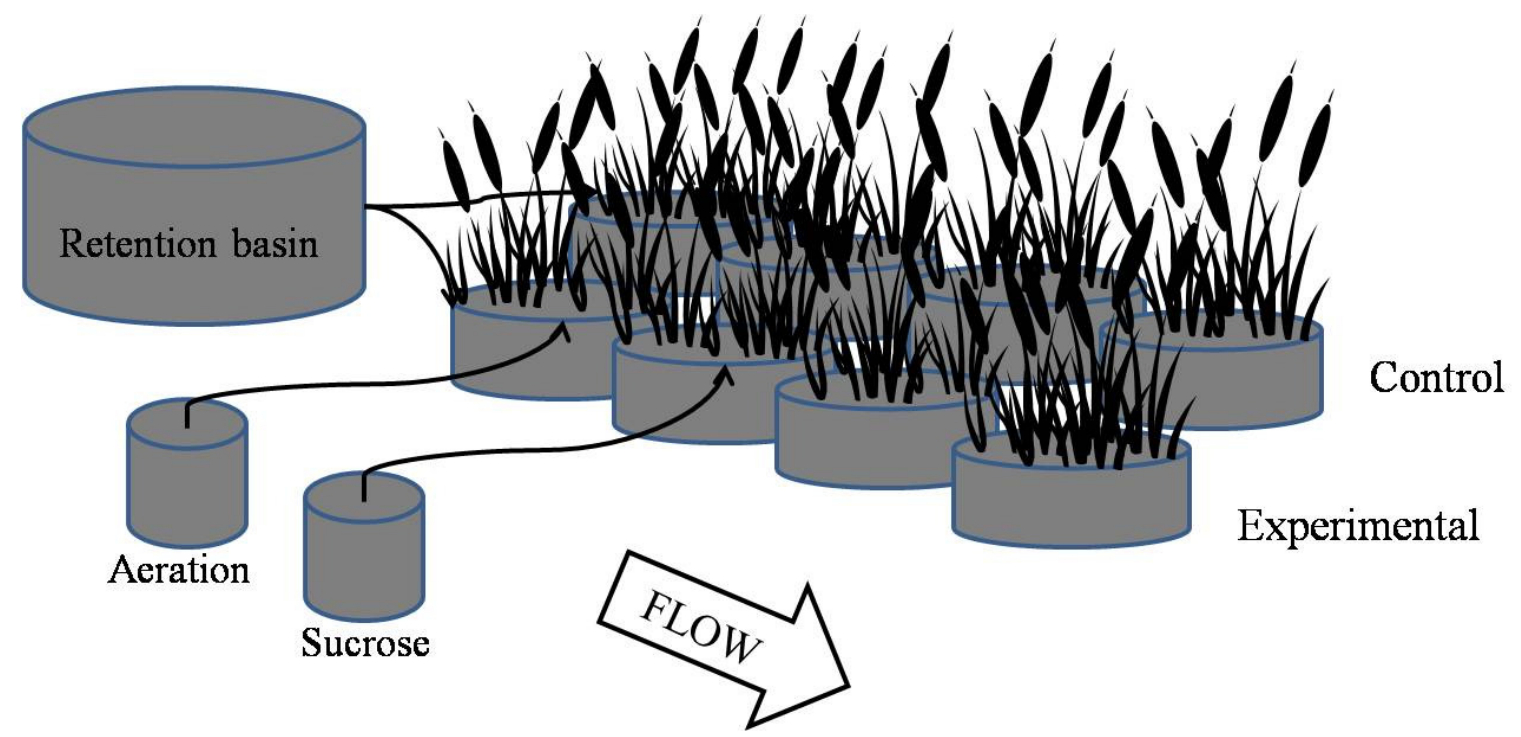

Figure 2 Schematic diagram of the pilot-scale CWTS for simulated post-RO PW

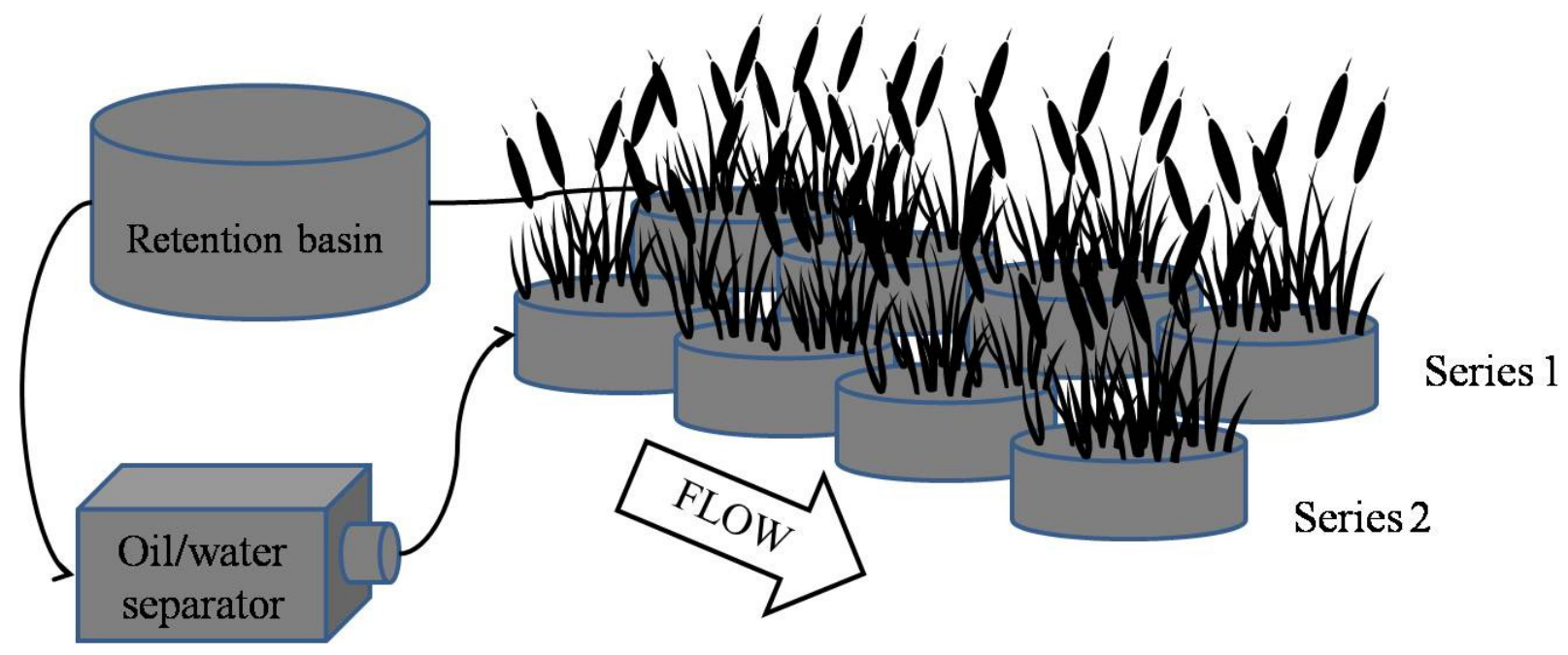

Figure 3 Schematic diagram of the pilot-scale CWTS for simulated PW containing divalent metals and LMWOs 


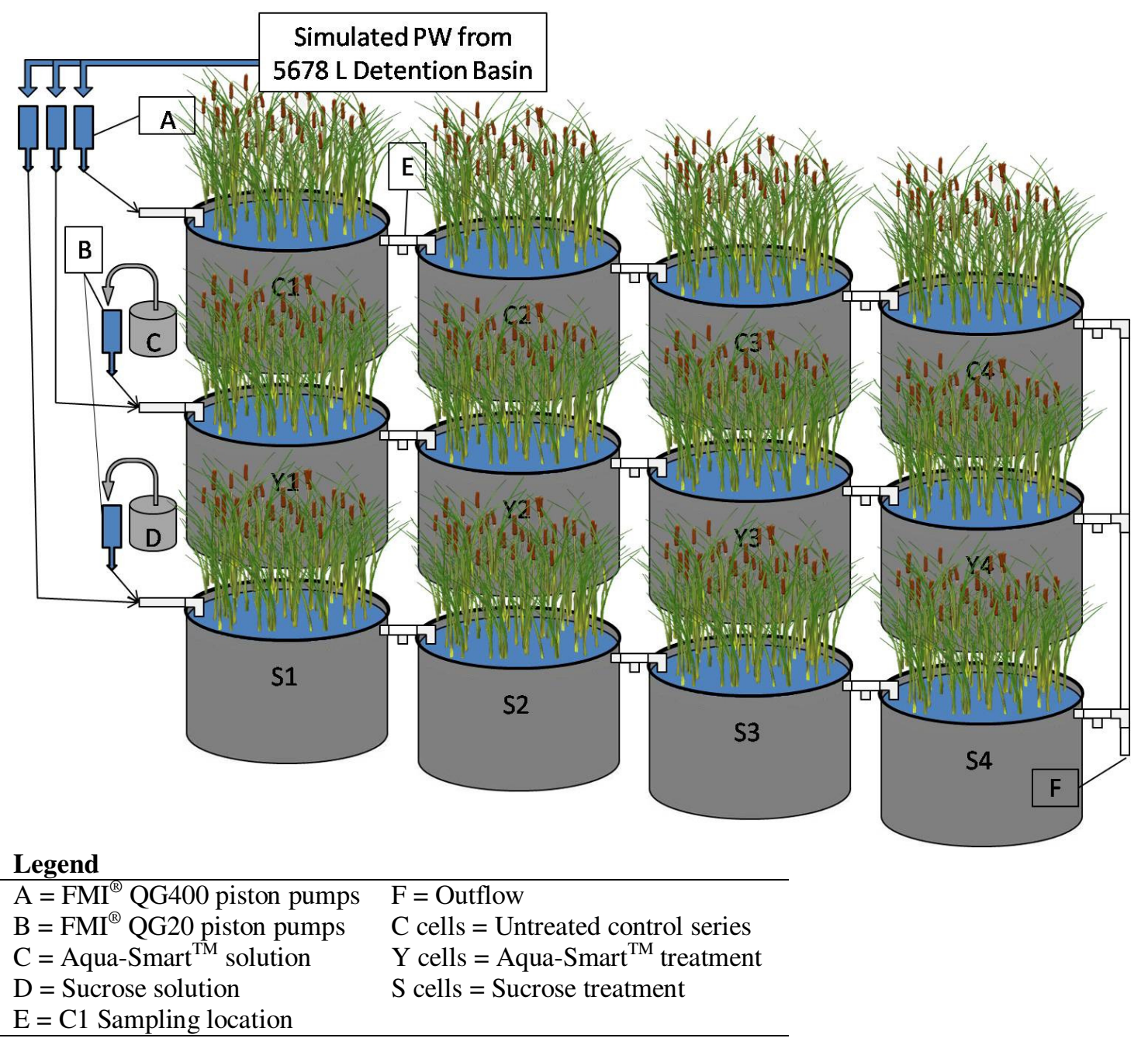

Figure 4 Schematic diagram of the pilot-scale CWTS for simulated PW containing metalloids and LMWOs 


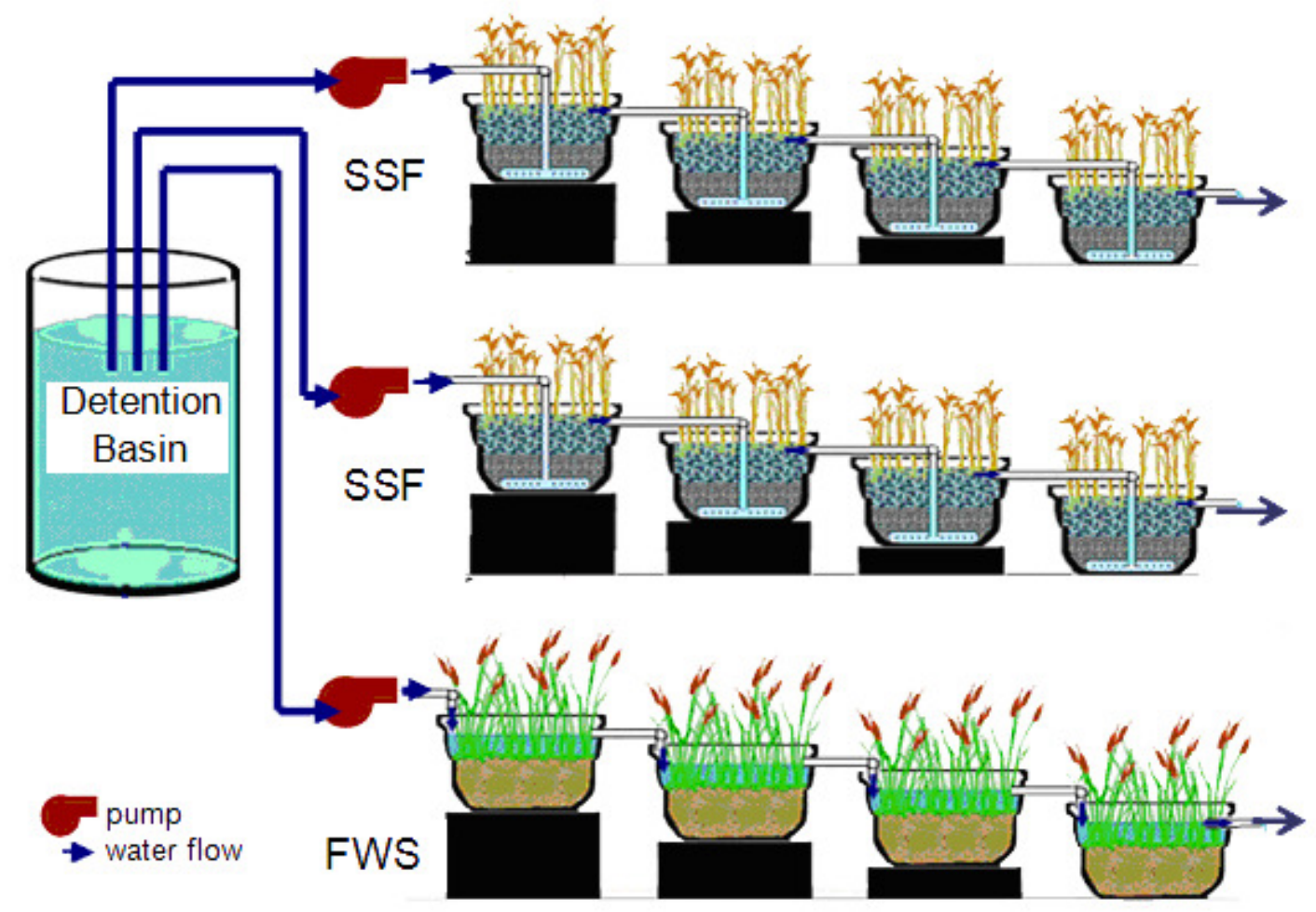

Figure 5 Schematic of the pilot-scale constructed wetland system constructed for simulated PW containing oil and grease. Wetland cells in subsurface flow (SSF) series are planted with Phragmites australis; free water surface (FWS) series is planted with Typha latifolia.

\section{$\underline{\text { Measure Treatment Performance of Pilot-Scale Constructed Wetland Systems (Task 5) }}$}

The objective of Task 5 is to measure performance of the pilot-scale systems designed and constructed during Task 4. Performance for each system is defined as the rate and extent of removal of targeted COCs. To determine the rate and extent of removal, strategic sampling and analyses for COC concentrations from sampling ports located after each cell was required. In addition, explanatory water chemistry parameters were monitored along with performance to determine whether targeted conditions for treatment pathways identified in Task 4 were met. These explanatory water chemistry parameters are in many cases directly related to the performance of the systems and are therefore referred to as explanatory parameters.

During a sampling period, water samples were collected from the influent of the systems and effluent of each cell along the treatment path. These samples were collected on a time interval equal to the designed HRT to follow the same volume of water through the length of the system. For example, with a 24 hour HRT, the first sampling day of a sampling period consisted of sampling the influent. The effluent of the first cell is sampled 24 hours later. This 24 hour sampling interval was followed until sampling of the final effluent is completed. The interval sampling method is essential for calculating accurate rate coefficients. 
All samples collected from the pilot scale CWTSs were brought to the laboratory at Clemson University for immediate analysis. Performance measurements are dependent upon the COC being treated and are discussed within the individual performance sections for each system. Explanatory parameters measured for each system included $\mathrm{pH}$, conductivity, alkalinity, hardness, DO, temperature, and redox potential. Table 24 lists the methods used for explanatory parameter analysis.

Table 24. Analytical methods for explanatory parameters in the pilot scale CWTSs

\begin{tabular}{lll}
\hline Parameter & Method & Detection Limit \\
\hline $\mathrm{pH}$ & Direct Instrumentation: Orion Model 420A & $0.01 \mathrm{~S} . \mathrm{U}$. \\
Conductivity & Direct Instrumentation: YSI 30 & $0.1 \mu \mathrm{S} / \mathrm{cm}$ \\
Alkalinity & Standard Methods: 2320 B & $2 \mathrm{mg} / \mathrm{L} \mathrm{as} \mathrm{CaCO3}$ \\
Hardness & Standard Methods: 2340 B & $2 \mathrm{mg} / \mathrm{L} \mathrm{as} \mathrm{CaCO3}$ \\
DO & Direct Instrumentation: YSI Model 52 & $0.1 \mathrm{mg} / \mathrm{L}$ \\
Temperature & Direct Instrumentation: YSI Model 52 & $0.5^{\circ} \mathrm{C}$ \\
Redox Potential & Modified standard method 2580B: GDT-11 Multi-meter & $10 \mathrm{mV}$ \\
\hline
\end{tabular}

Following completion of a sampling period, the extent of performance, or COC concentrations in each system's effluents, was compared with use criteria to determine whether treatment goals were being met.

\section{Pilot-Scale CWTS for Simulated Post-RO Produced Water}

Performance of the pilot scale CWTS for treating post-RO produced water was measured by determining the concentrations of both ammonia and nitrate. Although ammonia is initially the only COC present in the simulated post-RO produced water, nitrification leads to production of nitrate which may then become a COC. The treatment goals of this system were selected to make the simulated post-RO produced water suitable for irrigation. Therefore, effluent concentrations of ammonia must be below $5 \mathrm{mg} / \mathrm{L}$ and concentrations of nitrate must be below $10 \mathrm{mg} / \mathrm{L}$. These guidelines are based on the FAO guidelines for typical irrigation waters (Ayers and Westcott, 1994). In addition, although there is no regulation for ammonia concentration for domestic use of waters in the United States, the European Union recommends a concentration of no more than $0.5 \mathrm{mg} / \mathrm{L}$ ammonia and no more than $50 \mathrm{mg} / \mathrm{L}$ nitrate (European Union, 1998). Although the system is designed to treat post-RO produced water for crop irrigation, domestic use could also be considered.

For performance measurements, samples were collected following the sampling interval outlined above (24h HRT for the control system and 48h HRT for the experimental system) from both the control (Ctrl) and experimental systems (Exp). Laboratory analysis of both ammonia and nitrate were performed using standard methods (Table 25). Results for six sampling periods are shown in Tables 26-31. 
Table 25. Analytical methods for determining performance parameters in pilot scale CWTS for treating postRO produced water

\begin{tabular}{lllll}
\hline Parameters & Method & $\begin{array}{l}\text { Method } \\
\text { Detection Limit }\end{array}$ & $\begin{array}{l}\text { Initial } \\
\text { Concentration }\end{array}$ & $\begin{array}{l}\text { Target } \\
\text { Concentration }\end{array}$ \\
\hline Ammonia & $\begin{array}{l}\text { Standard Method:45000-NH3 D } \\
\text { (Ammonia ISE) }\end{array}$ & $0.1 \mathrm{mg} / \mathrm{L}$ & $20 \mathrm{mg} / \mathrm{L}$ & $5 \mathrm{mg} / \mathrm{L}$ \\
Nitrate & $\begin{array}{l}\text { Colorimetric Method (HACK- } \\
\text { modified from Standard } \\
\text { Methods:4500-N03 C) }\end{array}$ & $1 \mathrm{mg} / \mathrm{L}$ & $10 \mathrm{mg} / \mathrm{L}$ \\
\hline
\end{tabular}

Table 26. Ammonia and nitrate analysis and explanatory parameters for Sampling Period 1

\begin{tabular}{|c|c|c|c|c|c|c|c|c|c|}
\hline \multirow[b]{2}{*}{ Sample } & \multicolumn{2}{|c|}{ Performance Parameters } & \multicolumn{7}{|c|}{ Explanatory Parameters } \\
\hline & $\begin{array}{c}\text { [Ammonia-N] } \\
(\mathrm{mg} / \mathrm{L})\end{array}$ & $\begin{array}{c}{[\text { Nitrate-N] }} \\
(\mathrm{mg} / \mathrm{L})\end{array}$ & $\begin{array}{c}\mathrm{DO} \\
(\mathrm{mg} / \mathrm{L})\end{array}$ & $\begin{array}{l}\text { Cond. } \\
(\mu \mathrm{S} / \mathrm{cm})\end{array}$ & $\begin{array}{c}\mathrm{pH} \\
\text { (S.U.) }\end{array}$ & $\begin{array}{l}\text { Redox } \\
(\mathrm{mV})\end{array}$ & $\begin{array}{c}\text { Temp } \\
\left({ }^{\circ} \mathrm{C}\right)\end{array}$ & $\begin{array}{c}\text { Alkalinity } \\
(\mathrm{mg} / \mathrm{L} \\
\left.\mathrm{CaCO}_{3}\right)\end{array}$ & $\begin{array}{c}\text { Hardness } \\
(\mathrm{mg} / \mathrm{L} \\
\left.\mathrm{CaCO}_{3}\right)\end{array}$ \\
\hline Inflow & 20.9 & $<1$ & 11.0 & 355 & 8.14 & $\mathrm{nd}^{*}$ & 27 & 28 & 18 \\
\hline Ctrl 1 & 18.9 & $<1$ & 2.79 & 424 & 6.50 & nd & 26 & 44 & 36 \\
\hline Ctrl 2 & 17.6 & 2 & 3.01 & 430 & 6.05 & nd & 28 & 50 & 56 \\
\hline Ctrl 3 & 17.6 & 3 & 1.54 & 422 & 6.13 & nd & 27 & 50 & 60 \\
\hline Ctrl 4 & 17.6 & 4 & 1.32 & 434 & 6.17 & nd & 28 & 52 & 66 \\
\hline Exp 1 & 16.8 & $<1$ & 1.76 & 609 & 6.82 & nd & 26 & 76 & 178 \\
\hline $\operatorname{Exp} 2$ & 10.1 & 2 & 1.53 & 645 & 7.22 & nd & 28 & 180 & 194 \\
\hline Exp 3 & 3.8 & $<1$ & 0.35 & 592 & 6.92 & nd & 27 & 166 & 172 \\
\hline Exp 4 & 2.3 & 7 & 0.24 & 575 & 6.92 & nd & 28 & 164 & 184 \\
\hline
\end{tabular}

* nd-not determined

Table 27. Ammonia and nitrate analysis and explanatory parameters for Sampling Period 2

\begin{tabular}{|c|c|c|c|c|c|c|c|c|c|}
\hline \multirow[b]{2}{*}{ Sample } & \multicolumn{2}{|c|}{ Performance Parameters } & \multicolumn{7}{|c|}{ Explanatory Parameters } \\
\hline & $\begin{array}{c}{[\text { Ammonia-N] }} \\
(\mathrm{mg} / \mathrm{L})\end{array}$ & $\begin{array}{c}{[\text { Nitrate-N] }} \\
(\mathrm{mg} / \mathrm{L})\end{array}$ & $\begin{array}{c}\mathrm{DO} \\
(\mathrm{mg} / \mathrm{L}) \\
\end{array}$ & $\begin{array}{l}\text { Cond. } \\
(\mu \mathrm{S} / \mathrm{cm})\end{array}$ & $\begin{array}{c}\mathrm{pH} \\
\text { (S.U.) } \\
\end{array}$ & $\begin{array}{c}\text { Redox } \\
(\mathrm{mV})\end{array}$ & $\begin{array}{c}\text { Lab } \\
\text { Temp } \\
\left({ }^{\circ} \mathrm{C}\right) \\
\end{array}$ & $\begin{array}{c}\text { Alkalinity } \\
(\mathrm{mg} / \mathrm{L} \\
\left.\mathrm{CaCO}_{3}\right) \\
\end{array}$ & $\begin{array}{c}\text { Hardness } \\
(\mathrm{mg} / \mathrm{L} \\
\left.\mathrm{CaCO}_{3}\right) \\
\end{array}$ \\
\hline Inflow & 21.2 & $<1$ & 6.01 & 249 & 6.01 & $\mathrm{nd} *$ & 25 & 16 & 14 \\
\hline Ctrl 1 & 14.4 & $<1$ & 4.12 & 425 & 6.62 & -194.9 & 26 & 20 & 16 \\
\hline Ctrl 2 & 12.9 & $<1$ & 4.21 & 441 & 5.84 & -133 & 21 & 20 & 26 \\
\hline Ctrl 3 & 13.7 & $<1$ & 3.99 & 441 & 5.49 & 100.4 & 22 & 10 & 34 \\
\hline Ctrl 4 & 16.7 & $<1$ & 5.08 & 426 & 5.42 & 111.6 & 21 & 12 & 32 \\
\hline Exp 1 & 7.8 & 1 & 2.42 & 549 & 6.45 & -112.2 & 26 & 60 & 128 \\
\hline $\operatorname{Exp} 2$ & 7.4 & 2 & 3.17 & 670 & 6.28 & -46.2 & 22 & 110 & 124 \\
\hline Exp 3 & 8.0 & 4 & 2.66 & 720 & 6.43 & -36.3 & 20 & 192 & 280 \\
\hline Exp 4 & 7.8 & 4 & 2.33 & 760 & 6.51 & 68.4 & 20 & 212 & 304 \\
\hline
\end{tabular}

* nd - not determined 
Table 28. Ammonia and nitrate analysis and explanatory parameters for Sampling Period 3

\begin{tabular}{|c|c|c|c|c|c|c|c|c|c|}
\hline \multirow[b]{2}{*}{ Sample } & \multicolumn{2}{|c|}{ Performance Parameters } & \multicolumn{7}{|c|}{ Explanatory Parameters } \\
\hline & $\begin{array}{c}{[\text { Ammonia-N] }} \\
(\mathrm{mg} / \mathrm{L})\end{array}$ & $\begin{array}{c}\text { [Nitrate-N] } \\
(\mathrm{mg} / \mathrm{L})\end{array}$ & $\begin{array}{c}\mathrm{DO} \\
(\mathrm{mg} / \mathrm{L}) \\
\end{array}$ & $\begin{array}{l}\text { Cond. } \\
(\mu \mathrm{S} / \mathrm{cm})\end{array}$ & $\begin{array}{c}\mathrm{pH} \\
\text { (S.U.) }\end{array}$ & $\begin{array}{c}\text { Redox } \\
(\mathrm{mV})\end{array}$ & $\begin{array}{c}\text { Temp } \\
\left({ }^{\circ} \mathrm{C}\right)\end{array}$ & $\begin{array}{c}\text { Alkalinity } \\
(\mathrm{mg} / \mathrm{L} \\
\left.\mathrm{CaCO}_{3}\right) \\
\end{array}$ & $\begin{array}{c}\text { Hardness } \\
(\mathrm{mg} / \mathrm{L} \\
\left.\mathrm{CaCO}_{3}\right)\end{array}$ \\
\hline Inflow & 20.3 & $<1$ & 6.91 & 237 & 7.00 & $\mathrm{nd}^{*}$ & 14 & 14 & 16 \\
\hline Ctrl 1 & 18.2 & $<1$ & 3.20 & 236 & 6.08 & -156 & 12 & 8 & 20 \\
\hline Ctrl 2 & 17.2 & $<1$ & 2.87 & 234 & 5.58 & 24 & 11 & 8 & 22 \\
\hline Ctrl 3 & 16.4 & $<1$ & 2.45 & 233 & 4.80 & 61 & 11 & 6 & 24 \\
\hline Ctrl 4 & 14.3 & $<1$ & 3.80 & 235 & 5.22 & -27 & 11 & 10 & 32 \\
\hline Exp 1 & 15.9 & $<1$ & 4.57 & 247 & 6.17 & -263 & 12 & 8 & 34 \\
\hline Exp 2 & 10.3 & $<1$ & 1.29 & 292 & 6.08 & -260 & 13 & 50 & 92 \\
\hline Exp 3 & 10.3 & $<1$ & 1.90 & 256 & 6.20 & -190 & 11 & 56 & 108 \\
\hline Exp 4 & 7.4 & $<1$ & 1.90 & 309 & 6.41 & -17 & 11 & 96 & 140 \\
\hline
\end{tabular}

* nd - not determined

Table 29. Ammonia and nitrate analysis and explanatory parameters for Sampling Period 4

\begin{tabular}{|c|c|c|c|c|c|c|c|c|c|}
\hline \multirow[b]{2}{*}{ Sample } & \multicolumn{2}{|c|}{ Performance Parameters } & \multicolumn{7}{|c|}{ Explanatory Parameters } \\
\hline & $\begin{array}{c}{[\text { Ammonia-N] }} \\
(\mathrm{mg} / \mathrm{L})\end{array}$ & $\begin{array}{c}{[\text { Nitrate-N] }} \\
(\mathrm{mg} / \mathrm{L})\end{array}$ & $\begin{array}{c}\mathrm{DO} \\
(\mathrm{mg} / \mathrm{L}) \\
\end{array}$ & $\begin{array}{l}\text { Cond. } \\
(\mu \mathrm{S} / \mathrm{cm})\end{array}$ & $\begin{array}{c}\mathrm{pH} \\
\text { (S.U.) } \\
\end{array}$ & $\begin{array}{c}\text { Redox } \\
(\mathrm{mV})\end{array}$ & $\begin{array}{c}\text { Temp } \\
\left({ }^{\circ} \mathrm{C}\right) \\
\end{array}$ & $\begin{array}{c}\text { Alkalinity } \\
(\mathrm{mg} / \mathrm{L} \\
\left.\mathrm{CaCO}_{3}\right) \\
\end{array}$ & $\begin{array}{c}\text { Hardness } \\
(\mathrm{mg} / \mathrm{L} \\
\left.\mathrm{CaCO}_{3}\right) \\
\end{array}$ \\
\hline Inflow & 22.3 & $<1.0$ & 6.95 & 347 & 6.93 & $\mathrm{nd}^{*}$ & 19 & 16 & 18 \\
\hline Ctrl 1 & 18.3 & $<1.0$ & 4.85 & 332 & 5.01 & 18 & 16 & 10 & 28 \\
\hline Ctrl 2 & 16.5 & $<1.0$ & 4.01 & 349 & 4.73 & -202 & 16 & 6 & 26 \\
\hline Ctrl 3 & 14.2 & $<1.0$ & 5.64 & 333 & 5.01 & -51 & 16 & 8 & 28 \\
\hline Ctrl 4 & 12.6 & $<1.0$ & 2.99 & 364 & 5.13 & 47 & 16 & 6 & 34 \\
\hline Exp 1 & 14.6 & 4 & 6.43 & 362 & 5.56 & -291 & 16 & 10 & 74 \\
\hline $\operatorname{Exp} 2$ & 1.4 & 9 & 2.43 & 379 & 5.62 & -277 & 16 & 68 & 174 \\
\hline Exp 3 & 0.2 & 6 & 2.60 & 390 & 6.12 & -143 & 15 & 72 & 140 \\
\hline Exp 4 & $<0.1$ & 4 & 1.94 & 422 & 6.09 & -33 & 15 & 106 & 186 \\
\hline
\end{tabular}

* nd - not determined

Table 30. Ammonia and nitrate analysis and explanatory parameters for Sampling Period 5

\begin{tabular}{|c|c|c|c|c|c|c|c|c|c|}
\hline \multirow[b]{2}{*}{ Sample } & \multicolumn{2}{|c|}{ Performance Parameters } & \multicolumn{7}{|c|}{ Explanatory Parameters } \\
\hline & $\begin{array}{c}{[\text { Ammonia-N] }} \\
(\mathrm{mg} / \mathrm{L})\end{array}$ & $\begin{array}{c}{[\text { Nitrate-N] }} \\
(\mathrm{mg} / \mathrm{L})\end{array}$ & $\begin{array}{c}\mathrm{DO} \\
(\mathrm{mg} / \mathrm{L}) \\
\end{array}$ & $\begin{array}{l}\text { Cond. } \\
(\mu \mathrm{S} / \mathrm{cm})\end{array}$ & $\begin{array}{c}\mathrm{pH} \\
\text { (S.U.) } \\
\end{array}$ & $\begin{array}{c}\text { Redox } \\
(\mathrm{mV})\end{array}$ & $\begin{array}{c}\text { Temp } \\
\left({ }^{\circ} \mathrm{C}\right)\end{array}$ & $\begin{array}{c}\text { Alkalinity } \\
(\mathrm{mg} / \mathrm{L} \\
\left.\mathrm{CaCO}_{3}\right)\end{array}$ & $\begin{array}{c}\text { Hardness } \\
(\mathrm{mg} / \mathrm{L} \\
\left.\mathrm{CaCO}_{3}\right)\end{array}$ \\
\hline Inflow & 23.2 & $<1.0$ & 7.03 & 413 & 7.76 & $\mathrm{nd}^{*}$ & 25 & 12 & 14 \\
\hline Ctrl 1 & 20.3 & $<1.0$ & 1.5.0 & 419 & 5.01 & -24 & 25 & 10 & 16 \\
\hline $\mathrm{Ctrl} 2$ & 18.9 & 3 & 2.19 & 404 & 5.01 & -200 & 24 & 12 & 18 \\
\hline Ctrl 3 & 17.6 & 8 & 2.58 & 400 & 4.94 & -31 & 25 & 10 & 24 \\
\hline Ctrl 4 & 15.0 & 5 & 3.63 & 387 & 4.94 & 47 & 26 & 6 & 36 \\
\hline Exp 1 & 9.8 & 2 & 4.91 & 444 & 5.20 & -301 & 26 & 20 & 22 \\
\hline Exp 2 & 1.6 & 7 & 0.69 & 508 & 5.94 & -256 & 25 & 84 & 170 \\
\hline Exp 3 & $<0.1$ & 1 & 3.22 & 539 & 6.61 & -107 & 26 & 150 & 208 \\
\hline Exp 4 & $<0.1$ & 3 & 4.27 & 567 & 6.18 & 29 & 27 & 140 & 204 \\
\hline
\end{tabular}

* nd - not determined 
Table 31. Ammonia and nitrate analysis and explanatory parameters for Sampling Period 6

\begin{tabular}{|c|c|c|c|c|c|c|c|c|c|}
\hline \multirow[b]{2}{*}{ Sample } & \multicolumn{2}{|c|}{ Performance Parameters } & \multicolumn{7}{|c|}{ Explanatory Parameters } \\
\hline & $\begin{array}{c}{[\text { Ammonia-N] }} \\
(\mathrm{mg} / \mathrm{L})\end{array}$ & $\begin{array}{c}{[\text { Nitrate-N] }} \\
(\mathrm{mg} / \mathrm{L})\end{array}$ & $\begin{array}{c}\mathrm{DO} \\
(\mathrm{mg} / \mathrm{L})\end{array}$ & $\begin{array}{l}\text { Cond. } \\
(\mu \mathrm{S} / \mathrm{cm})\end{array}$ & $\begin{array}{c}\mathrm{pH} \\
\text { (S.U.) }\end{array}$ & $\begin{array}{l}\text { Redox } \\
(\mathrm{mV})\end{array}$ & $\begin{array}{l}\text { Temp } \\
\left({ }^{\circ} \mathrm{C}\right)\end{array}$ & $\begin{array}{c}\text { Alkalinity } \\
(\mathrm{mg} / \mathrm{L} \\
\left.\mathrm{CaCO}_{3}\right)\end{array}$ & $\begin{array}{c}\text { Hardness } \\
(\mathrm{mg} / \mathrm{L} \\
\left.\mathrm{CaCO}_{3}\right)\end{array}$ \\
\hline Inflow & 25.7 & $<1.0$ & 7.56 & 352 & 6.86 & $\mathrm{nd}^{*}$ & 29 & 22 & 16 \\
\hline Ctrl 1 & 19.2 & $<1.0$ & 4.07 & 348 & 5.00 & 28 & 28 & 10 & 24 \\
\hline Ctrl 2 & 17.2 & $<1.0$ & 2.65 & 356 & 5.46 & -165 & 28 & 18 & 42 \\
\hline Ctrl 3 & 15.6 & 2 & 2.31 & 367 & 5.33 & 42 & 28 & 14 & 50 \\
\hline Ctrl 4 & 13.2 & $<1.0$ & 3.68 & 387 & 5.21 & 67 & 28 & 16 & 56 \\
\hline Exp 1 & 4.4 & $<1.0$ & 5.71 & 373 & 5.31 & -254 & 28 & 10 & 100 \\
\hline $\operatorname{Exp} 2$ & 2.6 & 6 & 2.22 & 476 & 6.44 & -310 & 28 & 98 & 170 \\
\hline Exp 3 & $<0.1$ & 4 & 3.21 & 626 & 6.20 & -159 & 28 & 104 & 210 \\
\hline Exp 4 & $<0.1$ & 1 & 4.09 & 789 & 6.34 & 41 & 29 & 106 & 226 \\
\hline
\end{tabular}

* nd - not determined

For each of the sampling periods, the control system did not meet the targeted performance goal for ammonia. During sampling periods 2 and 3, the experimental system did not meet the targeted performance goal for ammonia; however, during sampling periods 1, 4, 5, and 6 the targeted performance goal was met (Figure 6). Sampling periods 2, 4, and 6 were excluded from the figure due to similar ammonia concentrations

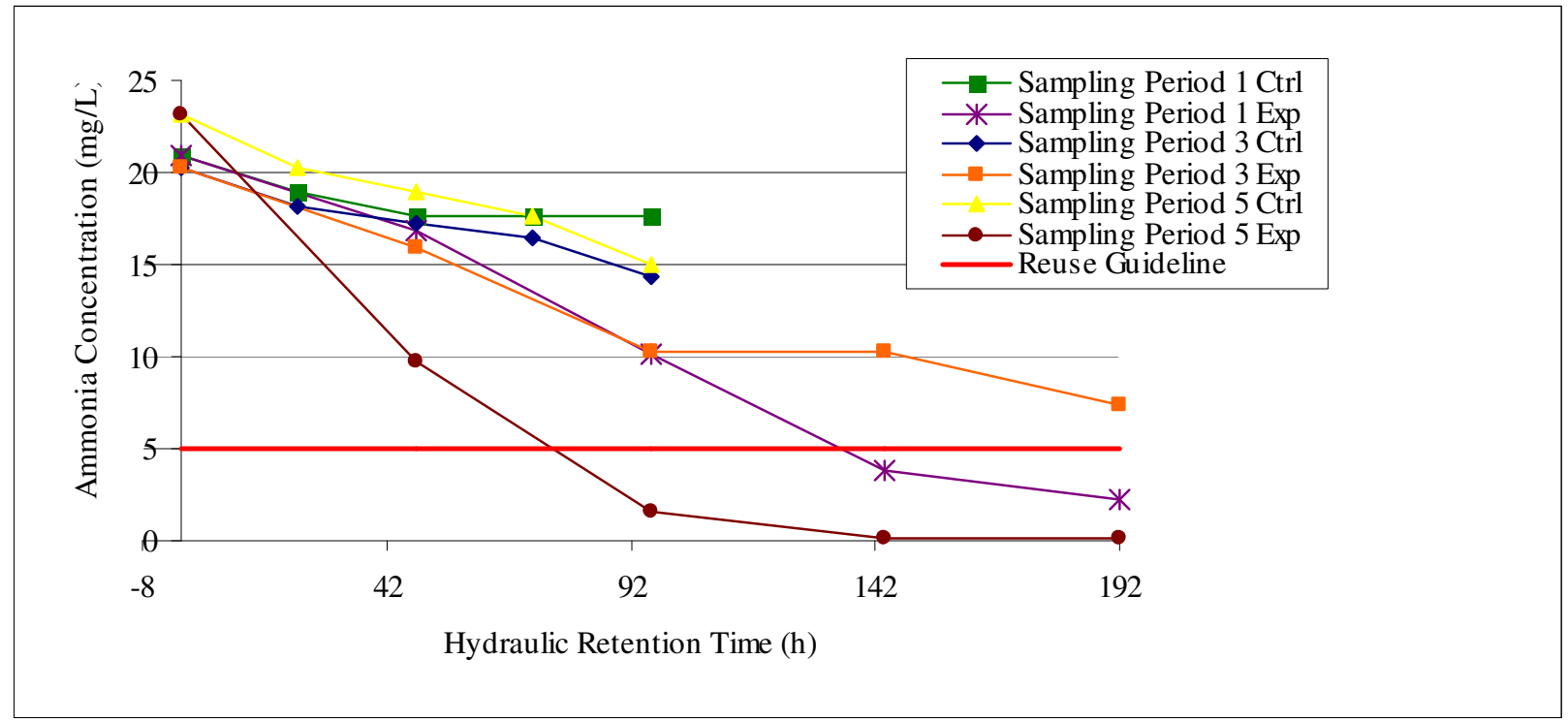

Figure 6. Ammonia removal during 3 sampling periods.

Sampling periods 2 and 3 were performed during cold winter months when freezing conditions negatively affected nitrification. This was demonstrated by simultaneously sampling the outdoor systems (Ctrl and Exp) during sampling period 3 and a pair of indoor control systems (Ictrl 1 and 2 ). These indoor systems were constructed with the same design parameters as the outdoor control system (24h HRT, cattails, identical water simulation, and fluvial hydrosoil) to establish similar environmental conditions excluding water and air temperatures. The steady water 
temperatures were obtained by placing the indoor control systems inside a climate controlled greenhouse. Results (Table 32) indicate that the outdoor control system was not able to obtain a level of performance comparable to that of the indoor control systems. Therefore, water and air temperatures are important considerations for design of a CWTS treating post-RO produced water.

Table 32. Ammonia and nitrate analysis and explanatory parameters for control systems located in a climate controlled greenhouse during Sampling Period 2

\begin{tabular}{lccccccccc}
\hline & \multicolumn{3}{c}{ Performance Parameters } & \multicolumn{1}{c}{ Explanatory Parameters } \\
\cline { 2 - 9 } Sample & $\begin{array}{c}\text { [Ammonia-N] } \\
(\mathrm{mg} / \mathrm{L})\end{array}$ & $\begin{array}{c}\text { [Nitrate-N] } \\
(\mathrm{mg} / \mathrm{L})\end{array}$ & $\begin{array}{c}\mathrm{DO} \\
(\mathrm{mg} / \mathrm{L})\end{array}$ & $\begin{array}{c}\text { Cond. } \\
(\mu \mathrm{HS} / \mathrm{cm})\end{array}$ & $\begin{array}{c}\mathrm{pH} \\
(\mathrm{S} . \mathrm{U} .)\end{array}$ & $\begin{array}{c}\text { Redox } \\
(\mathrm{mV})\end{array}$ & $\begin{array}{c}\text { Temp } \\
\left({ }^{\circ} \mathrm{C}\right)\end{array}$ & $\begin{array}{c}\text { Alkalinity } \\
(\mathrm{mg} / \mathrm{L} \\
\left.\mathrm{CaCO}_{3}\right)\end{array}$ & $\left.\begin{array}{c}\text { Hardness } \\
(\mathrm{mg} / \mathrm{L} \\
\mathrm{CaCO}\end{array}\right)$ \\
\hline Inflow & 21.9 & $<1$ & 5.95 & 241 & 7.73 & $\mathrm{Nd} *$ & 15 & 18 & 22 \\
Ictrl 1 & 10.7 & $<1$ & 3.68 & 332 & 5.79 & -187 & 15 & 34 & 98 \\
Ictrl 2 & 12.0 & $<1$ & 3.08 & 364 & 5.47 & -66 & 16 & 26 & 82 \\
\hline
\end{tabular}

nd - not determined

Nitrate concentrations were below the use guideline concentrations for all samples during all sampling periods.

\section{Pilot-Scale CWTS for PW Containing Divalent Metals and Low Molecular Weight Organics}

An outdoor pilot-scale CWTS was designed and constructed to remove $\mathrm{Cd}^{+2}, \mathrm{Cu}^{+2}, \mathrm{Ni}^{+2}, \mathrm{Zn}^{+2}$, and LMWOs. Composition of the simulated water is similar to that of fresh shale gas produced water (McIntosh et al. 2002). Performance of this system is defined as the rate and extent of removal of COCs. Performance was measured by an Inductively Coupled Plasma-Atomic Emission Spectrometer (ICP-AES) for $\mathrm{Cd}^{+2}, \mathrm{Cu}^{+2}, \mathrm{Ni}^{+2}$, and $\mathrm{Zn}^{+2}$ concentrations (USEPA 1994a) and total LMWOs were measured using a spectrofluorometric technique modified from Zhou et al. (2009).

Targeted inflow concentrations of constituents in the simulated PW were $4 \mathrm{mg} / \mathrm{L} \mathrm{Cu}{ }^{+2}, 6 \mathrm{mg} / \mathrm{L}$ $\mathrm{Cd}^{+2}, 6 \mathrm{mg} / \mathrm{L} \mathrm{Ni}^{+2}, 6 \mathrm{mg} / \mathrm{L} \mathrm{Zn}^{+2}$, and $25 \mathrm{mg} / \mathrm{L}$ LMWOs. For sampling period 1, the initial

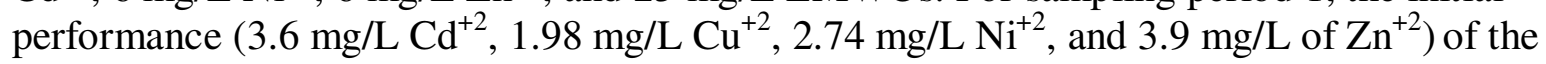
pilot-scale CWTS (Table 33 and Figures 7 and 8) was improved with the addition of calcium carbonate to the simulated PW during sampling period 2 (Table 34 and Figures 9 and 10) to provide buffering capacity (i.e. alkalinity) to the simulated water. Table 35 illustrates the differences in percent removal before and after the addition of calcium carbonate to the simulated PW. The improvement in performance with the addition of calcium carbonate may be due to maturity of the system, calcium carbonate providing buffering capacity for the simulated fresh PW to maintain a $\mathrm{pH}$ above 6 , and temperature increases $\left(\sim 15^{\circ} \mathrm{C}\right.$ to $24^{\circ} \mathrm{C}$; Tables $34,36-38$ and Figures 11-16), which may promote microbial activity. Microbial processes, such as dissimilatory sulfate reduction (for $\mathrm{Cd}^{+2}, \mathrm{Cu}^{+2}, \mathrm{Ni}^{+2}, \mathrm{Zn}^{+2}$ ) and biodegradation by heterotrophic aerobic microbes (for LMWOs) are the targeted removal pathways, and both may be temperature dependent (Abdollahi and Nedwell 1979; Halda-Alija and Johnston 1999). From sampling periods 2 to 5 for series 1, copper concentrations increased from $0.04 \mathrm{mg} / \mathrm{L}$ to $0.08 \mathrm{mg} / \mathrm{L}$; nickel concentrations decreased from $1.87 \mathrm{mg} / \mathrm{L}$ to $0.41 \mathrm{mg} / \mathrm{L}$; zinc concentrations decreased from 1.52 $\mathrm{mg} / \mathrm{L}$ to $0.08 \mathrm{mg} / \mathrm{L}$; and cadmium concentrations decreased from $0.82 \mathrm{mg} / \mathrm{L}$ to $0.2 \mathrm{mg} / \mathrm{L}$. Copper 
was the only element to increase from sampling period 2 to 5 for series 1 . From sampling periods 2 to 5 for series 2, copper concentrations decreased from $0.05 \mathrm{mg} / \mathrm{L}$ to $0.02 \mathrm{mg} / \mathrm{L}$; nickel concentrations from $1.60 \mathrm{mg} / \mathrm{L}$ to $0.04 \mathrm{mg} / \mathrm{L}$; zinc concentrations from $1.51 \mathrm{mg} / \mathrm{L}$ to $0.02 \mathrm{mg} / \mathrm{L}$; and cadmium concentrations from $0.79 \mathrm{mg} / \mathrm{L}$ to $0.09 \mathrm{mg} / \mathrm{L}$.

LMWOs concentrations were non-detect $(<1 \mathrm{mg} / \mathrm{L})$ after the first wetland cell. The goal of $1 \mathrm{mg}$ LMWOs/L in the outflow water has been achieved consistently by series 1 and 2 . The treatment goals to achieve specific water use criteria (i.e. irrigation and livestock watering) were accomplished for each of the four divalent metals and for LMWOs. 
Table 33. $\mathrm{Cd}^{+2}, \mathrm{Cu}^{+2}, \mathrm{Ni}^{+2}, \mathrm{Zn}^{+2}$, and LMWOs concentrations and explanatory parameters for Sampling Period 1

\begin{tabular}{|c|c|c|c|c|c|c|c|c|c|c|c|c|}
\hline \multirow[b]{2}{*}{ Sample } & \multicolumn{5}{|c|}{ Performance Parameters } & \multicolumn{7}{|c|}{ Explanatory Parameters } \\
\hline & $\begin{array}{l}\text { Copper } \\
(\mathrm{mg} / \mathrm{L})\end{array}$ & $\begin{array}{l}\text { Nickel } \\
(\mathrm{mg} / \mathrm{L})\end{array}$ & $\begin{array}{l}\text { Zinc } \\
(\mathrm{mg} / \mathrm{L})\end{array}$ & $\begin{array}{c}\text { Cadmium } \\
(\mathrm{mg} / \mathrm{L})\end{array}$ & $\begin{array}{c}\text { [LMWO] } \\
(\mathrm{mg} / \mathrm{L})\end{array}$ & $\begin{array}{c}\mathrm{DO} \\
(\mathrm{mg} / \mathrm{L})\end{array}$ & $\begin{array}{l}\text { Cond. } \\
(\mu \mathrm{S} / \mathrm{cm})\end{array}$ & $\begin{array}{c}\mathrm{pH} \\
\text { (S.U.) }\end{array}$ & $\begin{array}{c}\text { Redox } \\
(\mathrm{mV})\end{array}$ & $\begin{array}{l}\text { Temp } \\
\left({ }^{\circ} \mathrm{C}\right)\end{array}$ & $\begin{array}{c}\text { Alkalinity } \\
\text { (mg/L as } \\
\mathrm{CaCO}_{3} \text { ) }\end{array}$ & $\begin{array}{c}\text { Hardness } \\
(\mathrm{mg} / \mathrm{L} \text { as } \\
\left.\mathrm{CaCO}_{3}\right)\end{array}$ \\
\hline Inflow & 4 & 6.2 & 6.1 & 5.9 & bdl & 10.56 & 1732 & 6.75 & nd & 11.2 & 14 & 152 \\
\hline $\mathrm{T} 1-1$ & 3.4 & 5.7 & 5.4 & 6.15 & 0.35 & 9.06 & 1660 & 6.6 & -233 & 10.6 & 10 & 408 \\
\hline $\mathrm{T} 1-2$ & 3.1 & 4.9 & 4.8 & 5.61 & bdl & 7.69 & 1655 & 6.76 & -175 & 8.3 & 12 & 420 \\
\hline $\mathrm{T} 1-3$ & 2.4 & 2.9 & 4.1 & 3.9 & bdl & 11.76 & 1638 & 6.84 & -140 & 9.8 & 14 & 580 \\
\hline $\mathrm{T} 1-4$ & 1.98 & 2.74 & 3.9 & 3.6 & bdl & 9.01 & 1723 & 6.7 & 139 & 10.3 & 16 & 472 \\
\hline $\mathrm{T} 2-1$ & 4.12 & 6.2 & 5.85 & 4.8 & 0.65 & 7.51 & 1732 & 6.5 & -162 & 11.9 & 12 & 308 \\
\hline $\mathrm{T} 2-2$ & 2.55 & 6.1 & 5.7 & 4.2 & bdl & 6.16 & 1790 & 6.42 & -206 & 12.4 & 14 & 268 \\
\hline $\mathrm{T} 2-3$ & 2.37 & 5.9 & 5.1 & 3.9 & bdl & 9.64 & 1576 & 6.62 & -213 & 8.9 & 14 & 368 \\
\hline $\mathrm{T} 2-4$ & 2.43 & 5.8 & 4.5 & 3.75 & bdl & 10.2 & 1675 & 6.59 & -17.9 & 10.1 & 12 & 284 \\
\hline
\end{tabular}

nd- not determined

bdl- below detection limit 
Table 34. $\mathrm{Cd}^{+2}, \mathrm{Cu}^{+2}, \mathrm{Ni}^{+2}, \mathrm{Zn}^{+2}$, and LMWOs concentrations and explanatory parameters for Sampling Period 2

\begin{tabular}{|c|c|c|c|c|c|c|c|c|c|c|c|c|}
\hline \multirow[b]{2}{*}{ Sample } & \multicolumn{5}{|c|}{ Performance Parameters } & \multicolumn{7}{|c|}{ Explanatory Parameters } \\
\hline & $\begin{array}{l}\text { Copper } \\
(\mathrm{mg} / \mathrm{L})\end{array}$ & $\begin{array}{l}\text { Nickel } \\
(\mathrm{mg} / \mathrm{L})\end{array}$ & $\begin{array}{c}\text { Zinc } \\
(\mathrm{mg} / \mathrm{L})\end{array}$ & $\begin{array}{c}\text { Cadmium } \\
(\mathrm{mg} / \mathrm{L})\end{array}$ & $\begin{array}{c}\text { [LMWO] } \\
(\mathrm{mg} / \mathrm{L})\end{array}$ & $\begin{array}{c}\mathrm{DO} \\
(\mathrm{mg} / \mathrm{L})\end{array}$ & $\begin{array}{l}\text { Cond. } \\
(\mu \mathrm{S} / \mathrm{cm})\end{array}$ & $\begin{array}{c}\mathrm{pH} \\
\text { (S.U.) }\end{array}$ & $\begin{array}{c}\text { Redox } \\
(\mathrm{mV})\end{array}$ & $\begin{array}{l}\text { Temp } \\
\left({ }^{\circ} \mathrm{C}\right)\end{array}$ & $\begin{array}{c}\text { Alkalinity } \\
(\mathrm{mg} / \mathrm{L} \text { as } \\
\left.\mathrm{CaCO}_{3}\right)\end{array}$ & $\begin{array}{c}\text { Hardness } \\
(\mathrm{mg} / \mathrm{L} \text { as } \\
\left.\mathrm{CaCO}_{3}\right)\end{array}$ \\
\hline Inflow & 3.53 & 6.56 & 5.79 & 5.35 & bdl & 7.30 & 1838 & 6.91 & nd & 18.9 & 16 & 352 \\
\hline $\mathrm{T} 1-1$ & 0.21 & 1.99 & 1.49 & 1.46 & bdl & 7.81 & 1620 & 6.72 & -233 & 16.3 & 29 & 308 \\
\hline $\mathrm{T} 1-2$ & 0.12 & 2.32 & 1.86 & 1.36 & bdl & 4.64 & 1560 & 6.60 & -215 & 16.3 & 30 & 360 \\
\hline $\mathrm{T} 1-3$ & 0.15 & 2.45 & 2.06 & 1.51 & bdl & 9.39 & 1412 & 6.79 & -100 & 15.3 & 40 & 312 \\
\hline $\mathrm{T} 1-4$ & 0.04 & 1.87 & 1.52 & 0.82 & bdl & 8.23 & 1078 & 6.52 & 122 & 16.3 & 36 & 290 \\
\hline $\mathrm{T} 2-1$ & 0.26 & 1.69 & 1.42 & 0.99 & bdl & 8.12 & 1642 & 6.61 & -241 & 14.0 & 32 & 254 \\
\hline $\mathrm{T} 2-2$ & 0.12 & 2.49 & 2.40 & 1.58 & bdl & 7.59 & 1556 & 6.42 & -222 & 17.5 & 34 & 236 \\
\hline $\mathrm{T} 2-3$ & 0.13 & 2.43 & 2.39 & 1.43 & bdl & 6.79 & 1101 & 6.49 & -57 & 16.2 & 40 & 126 \\
\hline $\mathrm{T} 2-4$ & 0.05 & 1.60 & 1.51 & 0.79 & bdl & 6.79 & 799 & 6.48 & 118 & 15.9 & 40 & 276 \\
\hline
\end{tabular}

nd-not determined

bdl- below detection limit

Table 35. Percent removal of $\mathrm{Cd}^{+2}, \mathrm{Cu}^{+2}, \mathrm{Ni}^{+2}$, and $\mathrm{Zn}^{+2}$ before and after addition of calcium carbonate

\begin{tabular}{cccccccccc}
\hline Before $\mathrm{CaCO}_{3}$ & Copper & Cadmium & Nickel & Zinc & After $\mathrm{CaCO}_{3}$ & Copper & Cadmium & Nickel & Zinc \\
\hline Series 1 & $50.5 \%$ & $55.8 \%$ & $36.1 \%$ & $38.9 \%$ & Series 1 & $98.7 \%$ & $85.4 \%$ & $75.4 \%$ & $73.7 \%$ \\
Series 2 & $39.2 \%$ & $6.5 \%$ & $26.2 \%$ & $36.4 \%$ & Series 2 & $98.7 \%$ & $84.7 \%$ & $71.2 \%$ & $73.7 \%$ \\
\hline
\end{tabular}


Table 36. $\mathrm{Cd}^{+2}, \mathrm{Cu}^{+2}, \mathrm{Ni}^{+2}, \mathrm{Zn}^{+2}$, and LMWOs concentrations and explanatory parameters for Sampling Period 3

\begin{tabular}{|c|c|c|c|c|c|c|c|c|c|c|c|c|}
\hline \multirow[b]{2}{*}{ Sample } & \multicolumn{5}{|c|}{ Performance Parameters } & \multicolumn{7}{|c|}{ Explanatory Parameters } \\
\hline & $\begin{array}{l}\text { Copper } \\
(\mathrm{mg} / \mathrm{L})\end{array}$ & $\begin{array}{l}\text { Nickel } \\
(\mathrm{mg} / \mathrm{L})\end{array}$ & $\begin{array}{c}\text { Zinc } \\
(\mathrm{mg} / \mathrm{L})\end{array}$ & $\begin{array}{c}\text { Cadmium } \\
\text { (mg/L) }\end{array}$ & $\begin{array}{c}{[\mathrm{LMWO}]} \\
(\mathrm{mg} / \mathrm{L})\end{array}$ & $\begin{array}{c}\mathrm{DO} \\
(\mathrm{mg} / \mathrm{L})\end{array}$ & $\begin{array}{l}\text { Cond. } \\
(\mu \mathrm{S} / \mathrm{cm})\end{array}$ & $\begin{array}{c}\mathrm{pH} \\
\text { (S.U.) }\end{array}$ & $\begin{array}{c}\text { Redox } \\
(\mathrm{mV})\end{array}$ & $\begin{array}{l}\text { Temp } \\
\left({ }^{\circ} \mathrm{C}\right)\end{array}$ & $\begin{array}{c}\text { Alkalinity } \\
(\mathrm{mg} / \mathrm{L} \text { as } \\
\left.\mathrm{CaCO}_{3}\right)\end{array}$ & $\begin{array}{c}\text { Hardness } \\
(\mathrm{mg} / \mathrm{L} \text { as } \\
\left.\mathrm{CaCO}_{3}\right)\end{array}$ \\
\hline Inflow & 1.49 & 4.26 & 4.57 & 4.37 & 5.41 & 3.55 & 410 & 6.81 & nd & 24.2 & 32 & 542 \\
\hline $\mathrm{T} 1-1$ & 0.87 & 2.24 & 1.43 & 1.62 & 1.22 & 2.91 & 430 & 6.44 & -197.7 & 23.5 & 20 & 286 \\
\hline $\mathrm{T} 1-2$ & 0.73 & 0.68 & 0.58 & 0.34 & bdl & 2.25 & 594 & 6.69 & -118 & 23.9 & 42 & 236 \\
\hline $\mathrm{T} 1-3$ & 0.63 & 0.58 & 0.46 & 0.28 & bdl & 3.15 & 526 & 6.71 & -202 & 23.7 & 38 & 178 \\
\hline $\mathrm{T} 1-4$ & 0.54 & 0.61 & 1.22 & 0.25 & bdl & 2.53 & 596 & 6.67 & -72 & 22.9 & 42 & 144 \\
\hline $\mathrm{T} 2-1$ & 1.11 & 1.38 & 1.21 & 1.43 & 1.89 & 4.87 & 428 & 6.59 & -175.2 & 23.4 & 46 & 254 \\
\hline $\mathrm{T} 2-2$ & 1.41 & 0.89 & 0.38 & 0.25 & bdl & 2.51 & 448 & 6.63 & -164.2 & 23.8 & 52 & 174 \\
\hline T2-3 & 0.89 & 0.38 & 0.29 & 0.05 & bdl & 1.27 & 434 & 6.63 & -210 & 23.6 & 50 & 152 \\
\hline $\mathrm{T} 2-4$ & 0.06 & 0.09 & 0.09 & 0.01 & bdl & 1.59 & 473 & 6.71 & -124.9 & 23.7 & 36 & 122 \\
\hline
\end{tabular}

nd-not determined

bdl- below detection limit 
Table 37. $\mathrm{Cd}^{+2}, \mathrm{Cu}^{+2}, \mathrm{Ni}^{+2}, \mathrm{Zn}^{+2}$, and LMWOs concentrations and explanatory parameters for Sampling Period 4

\begin{tabular}{|c|c|c|c|c|c|c|c|c|c|c|c|c|}
\hline \multirow[b]{2}{*}{ Sample } & \multicolumn{5}{|c|}{ Performance Parameters } & \multicolumn{7}{|c|}{ Explanatory Parameters } \\
\hline & $\begin{array}{l}\text { Copper } \\
(\mathrm{mg} / \mathrm{L})\end{array}$ & $\begin{array}{l}\text { Nickel } \\
(\mathrm{mg} / \mathrm{L})\end{array}$ & $\begin{array}{l}\text { Zinc } \\
(\mathrm{mg} / \mathrm{L})\end{array}$ & $\begin{array}{c}\text { Cadmium } \\
(\mathrm{mg} / \mathrm{L})\end{array}$ & $\begin{array}{c}{[\mathrm{LMWO}]} \\
(\mathrm{mg} / \mathrm{L})\end{array}$ & $\begin{array}{c}\mathrm{DO} \\
(\mathrm{mg} / \mathrm{L})\end{array}$ & $\begin{array}{l}\text { Cond. } \\
(\mu \mathrm{S} / \mathrm{cm})\end{array}$ & $\begin{array}{c}\mathrm{pH} \\
\text { (S.U.) }\end{array}$ & $\begin{array}{l}\text { Redox } \\
(\mathrm{mV})\end{array}$ & $\begin{array}{l}\text { Temp } \\
\left({ }^{\circ} \mathrm{C}\right)\end{array}$ & $\begin{array}{c}\text { Alkalinity } \\
(\mathrm{mg} / \mathrm{L} \text { as } \\
\left.\mathrm{CaCO}_{3}\right)\end{array}$ & $\begin{array}{c}\text { Hardness } \\
(\mathrm{mg} / \mathrm{L} \text { as } \\
\left.\mathrm{CaCO}_{3}\right)\end{array}$ \\
\hline Inflow & 3.51 & 4.82 & 3.51 & 4.45 & 5.23 & 3.21 & 1691 & 7.04 & nd & 26.7 & 30 & 576 \\
\hline $\mathrm{T} 1-1$ & 1.16 & 3.63 & 2.48 & 2.28 & 0.17 & 2.09 & 1620 & 6.5 & -245.6 & 24.6 & 20 & 296 \\
\hline $\mathrm{T} 1-2$ & 1.94 & 2.17 & 1.19 & 0.12 & bdl & 1.91 & 1388 & 6.52 & -251.7 & 23.9 & 44 & 224 \\
\hline $\mathrm{T} 1-3$ & 1.1 & 1.28 & 0.52 & bdl & bdl & 1.91 & 1386 & 6.6 & -168 & 23.5 & 42 & 156 \\
\hline $\mathrm{T} 1-4$ & 1.49 & 1.31 & 0.44 & 2.09 & bdl & 1.92 & 1152 & 6.75 & -25 & 23.5 & 44 & 184 \\
\hline $\mathrm{T} 2-1$ & 0.88 & 0.63 & 0.39 & 0.75 & 1.14 & 2.47 & 1264 & 6.54 & -258 & 23.2 & 44 & 216 \\
\hline $\mathrm{T} 2-2$ & 0.92 & 0.59 & 0.41 & bdl & bdl & 2.05 & 1100 & 6.5 & -237 & 22.8 & 58 & 160 \\
\hline $\mathrm{T} 2-3$ & 0.053 & 0.12 & 0.12 & 0.07 & bdl & 1.95 & 862 & 6.45 & -144 & 23.4 & 52 & 136 \\
\hline T2-4 & 0.03 & 0.01 & 0.08 & bdl & bdl & 2.55 & 741 & 6.39 & -22 & 23.3 & 34 & 100 \\
\hline
\end{tabular}

nd-not determined

bdl- below detection limit 
Table 38. $\mathrm{Cd}^{+2}, \mathrm{Cu}^{+2}, \mathrm{Ni}^{+2}, \mathrm{Zn}^{+2}$, and LMWOs concentrations and explanatory parameters for Sampling Period 5

\begin{tabular}{|c|c|c|c|c|c|c|c|c|c|c|c|c|}
\hline \multirow[b]{2}{*}{ Sample } & \multicolumn{5}{|c|}{ Performance Parameters } & \multicolumn{7}{|c|}{ Explanatory Parameters } \\
\hline & $\begin{array}{l}\text { Copper } \\
(\mathrm{mg} / \mathrm{L})\end{array}$ & $\begin{array}{l}\text { Nickel } \\
(\mathrm{mg} / \mathrm{L})\end{array}$ & $\begin{array}{c}\text { Zinc } \\
(\mathrm{mg} / \mathrm{L})\end{array}$ & $\begin{array}{c}\text { Cadmium } \\
(\mathrm{mg} / \mathrm{L})\end{array}$ & $\begin{array}{c}\text { [LMWO] } \\
(\mathrm{mg} / \mathrm{L})\end{array}$ & $\begin{array}{c}\mathrm{DO} \\
(\mathrm{mg} / \mathrm{L})\end{array}$ & $\begin{array}{l}\text { Cond. } \\
(\mu \mathrm{S} / \mathrm{cm})\end{array}$ & $\begin{array}{c}\mathrm{pH} \\
\text { (S.U.) }\end{array}$ & $\begin{array}{l}\text { Redox } \\
(\mathrm{mV})\end{array}$ & $\begin{array}{l}\text { Temp } \\
\left({ }^{\circ} \mathrm{C}\right)\end{array}$ & $\begin{array}{l}\text { Alkalinity } \\
(\mathrm{mg} / \mathrm{L} \text { as } \\
\left.\mathrm{CaCO}_{3}\right)\end{array}$ & $\begin{array}{l}\text { Hardness } \\
(\mathrm{mg} / \mathrm{L} \text { as } \\
\left.\mathrm{CaCO}_{3}\right)\end{array}$ \\
\hline Inflow & 2.839 & 2.88 & 3.81 & 1.98 & 6.59 & 2.93 & 2014 & 7.02 & nd & 34.2 & 40 & 208 \\
\hline $\mathrm{T} 1-1$ & 1.958 & 1.51 & 1.96 & 0.65 & 1.611 & 2.64 & 1537 & 6.29 & -222.7 & 25.1 & 32 & 164 \\
\hline $\mathrm{T} 1-2$ & 0.296 & 1.58 & 0.30 & 0.34 & bdl & 1.41 & 1047 & 6.47 & -203 & 25.3 & 64 & 196 \\
\hline $\mathrm{T} 1-3$ & 0.1513 & 0.52 & 0.15 & 0.31 & bdl & 2.17 & 965 & 6.45 & -342 & 25.1 & 38 & 136 \\
\hline $\mathrm{T} 1-4$ & 0.084 & 0.41 & 0.08 & 0.02 & bdl & 1.56 & 946 & 6.43 & -289 & 24.7 & 26 & 100 \\
\hline $\mathrm{T} 2-1$ & 0.85 & 1.07 & 0.85 & 0.64 & 0.103 & 1.36 & 1295 & 6.65 & -169 & 24.5 & 82 & 152 \\
\hline $\mathrm{T} 2-2$ & 0.45 & 0.63 & 0.45 & 0.50 & bdl & 1.3 & 1179 & 6.46 & -177 & 24.5 & 80 & 212 \\
\hline T2-3 & 0.26 & 0.11 & 0.26 & 0.18 & bdl & 1.54 & 727 & 6.48 & -12 & 24.4 & 80 & 140 \\
\hline $\mathrm{T} 2-4$ & 0.02 & 0.04 & 0.02 & 0.09 & bdl & 2.15 & 756 & 6.51 & 13 & 24.6 & 44 & 104 \\
\hline
\end{tabular}

nd - not determined

bdl- below detection limit 


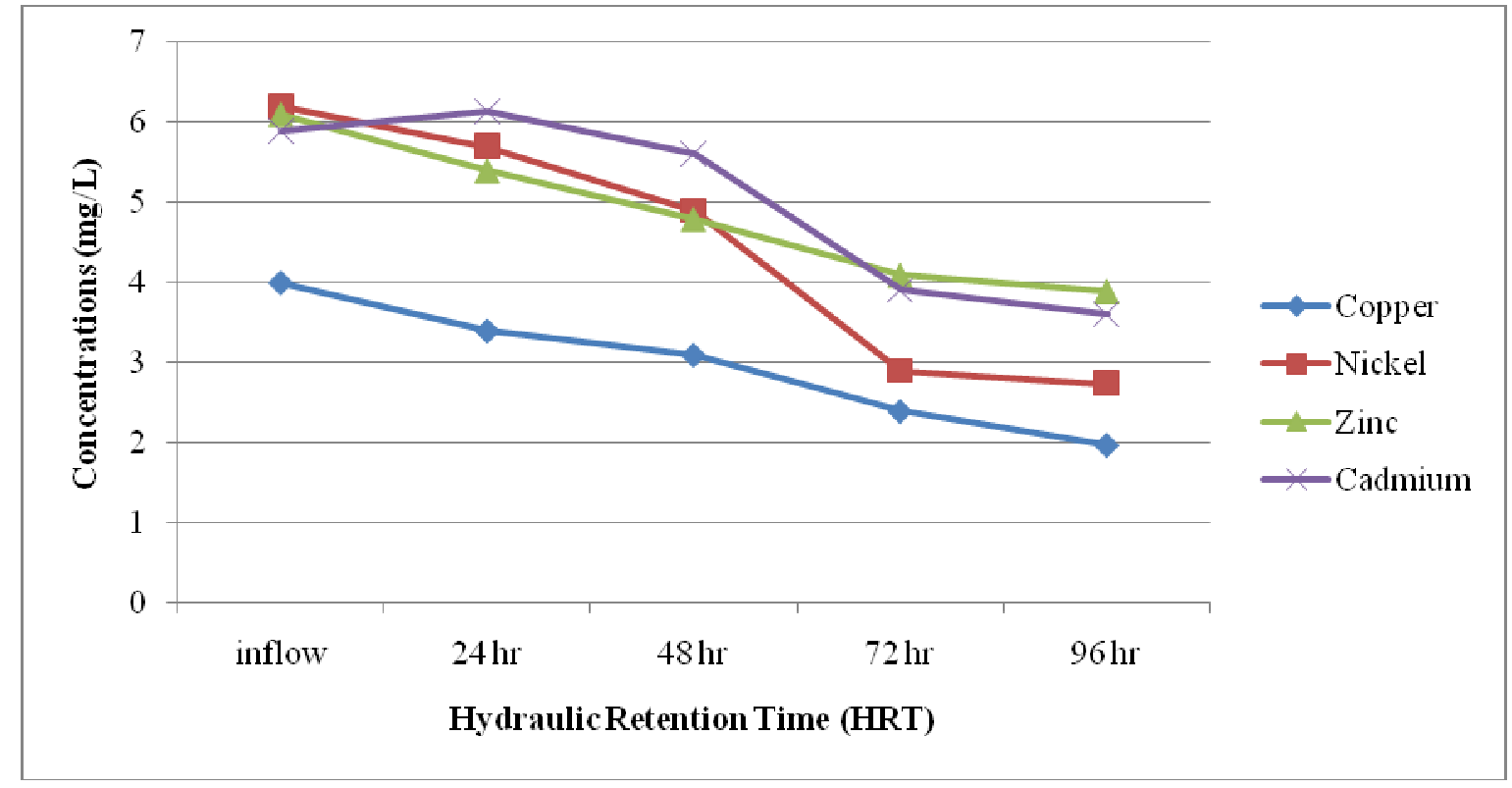

Figure 7. COC concentrations in series 1 during sampling period 1.

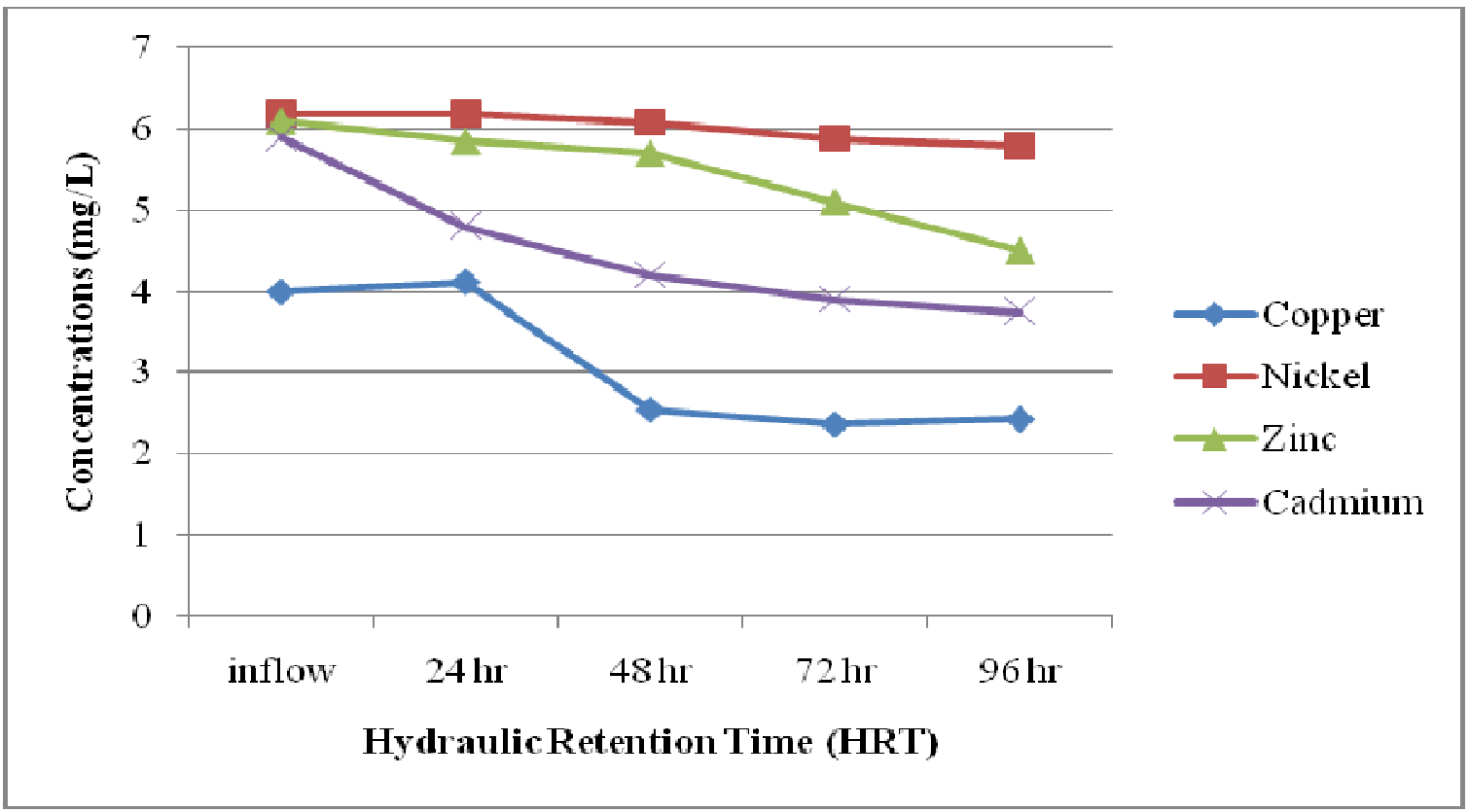

Figure 8. COC concentrations in series 2 during sampling period 1. 


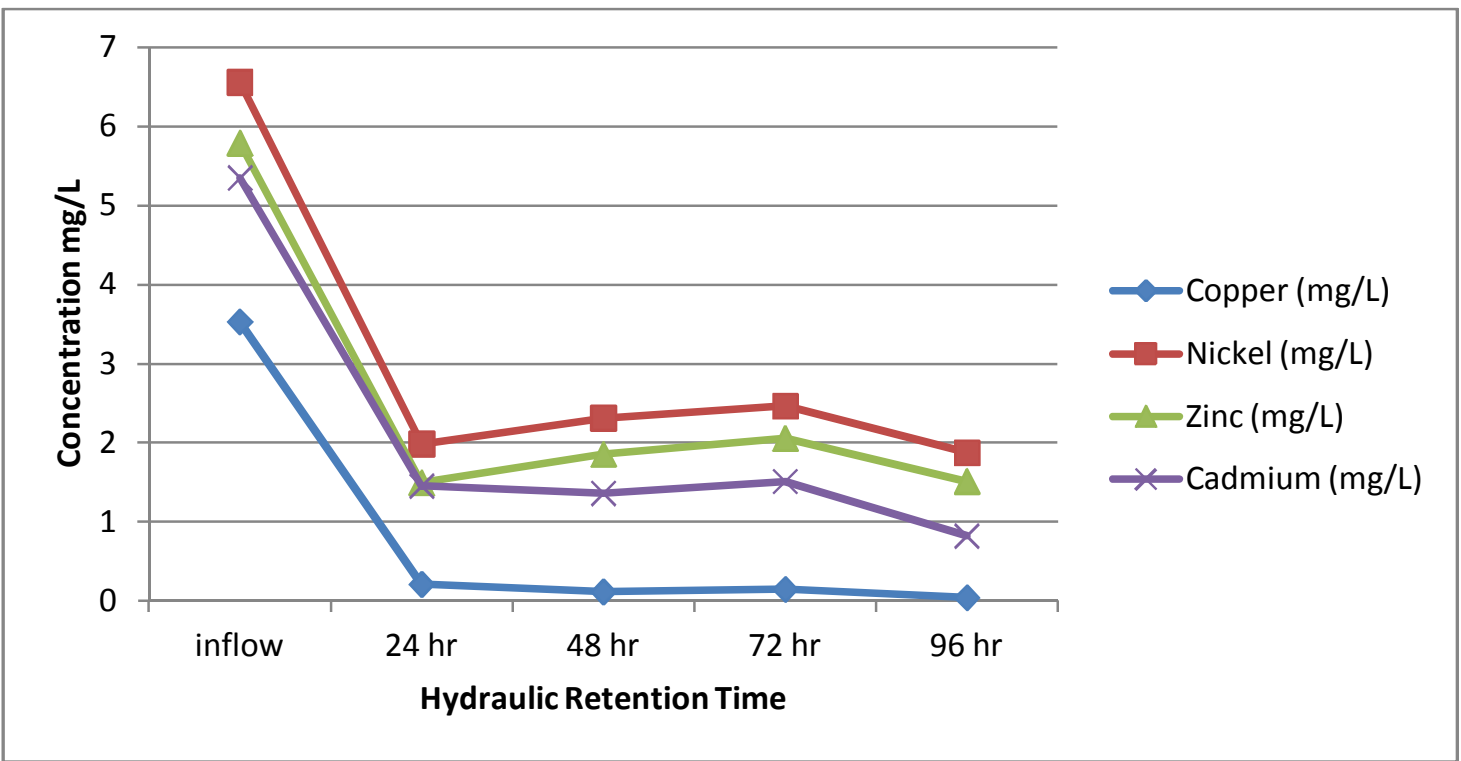

Figure 9. COC concentrations in series 1 after the addition of calcium carbonate salt to simulated fresh PW during sampling period 2.

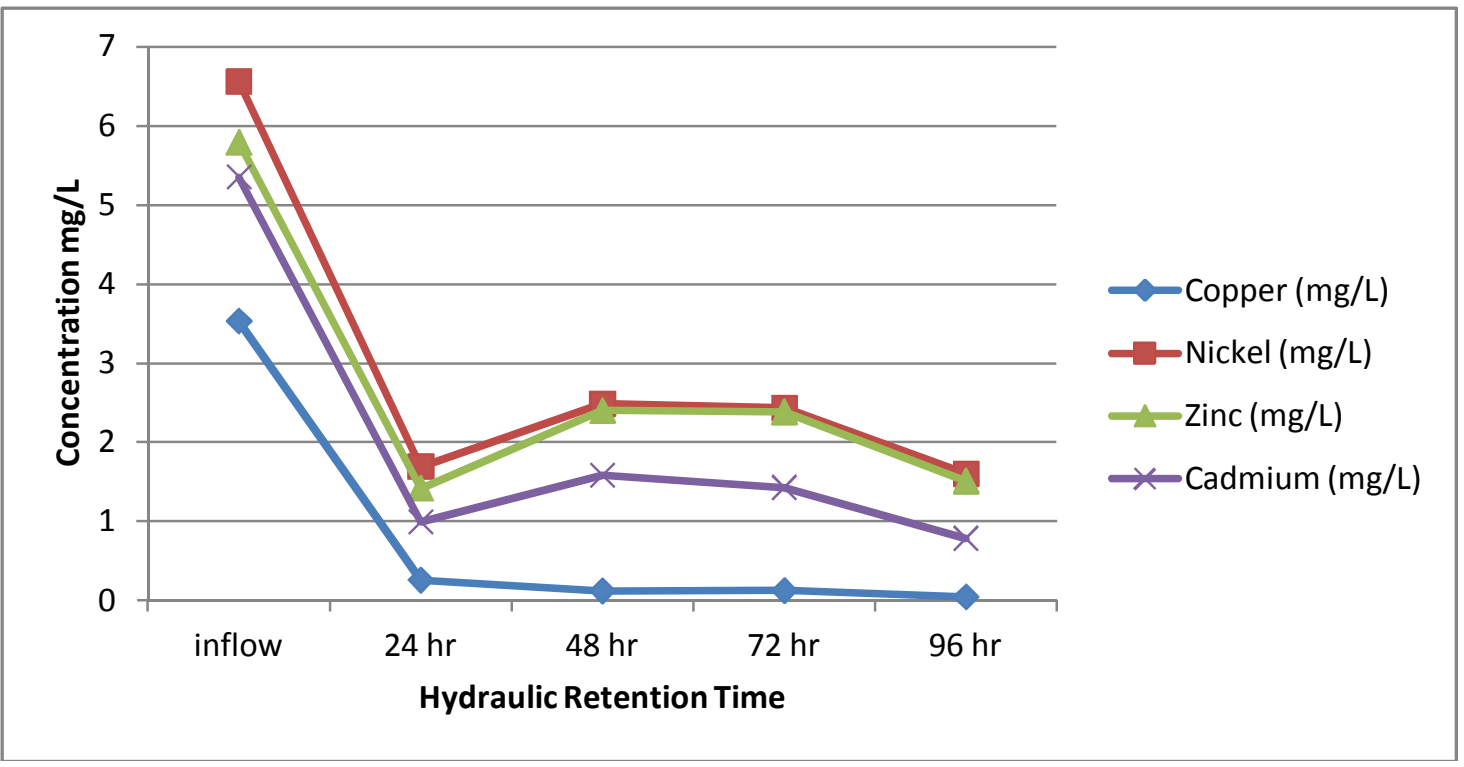

Figure 10. COC concentrations in series 2 after the addition of calcium carbonate salt to simulated fresh PW during sampling period 2. 


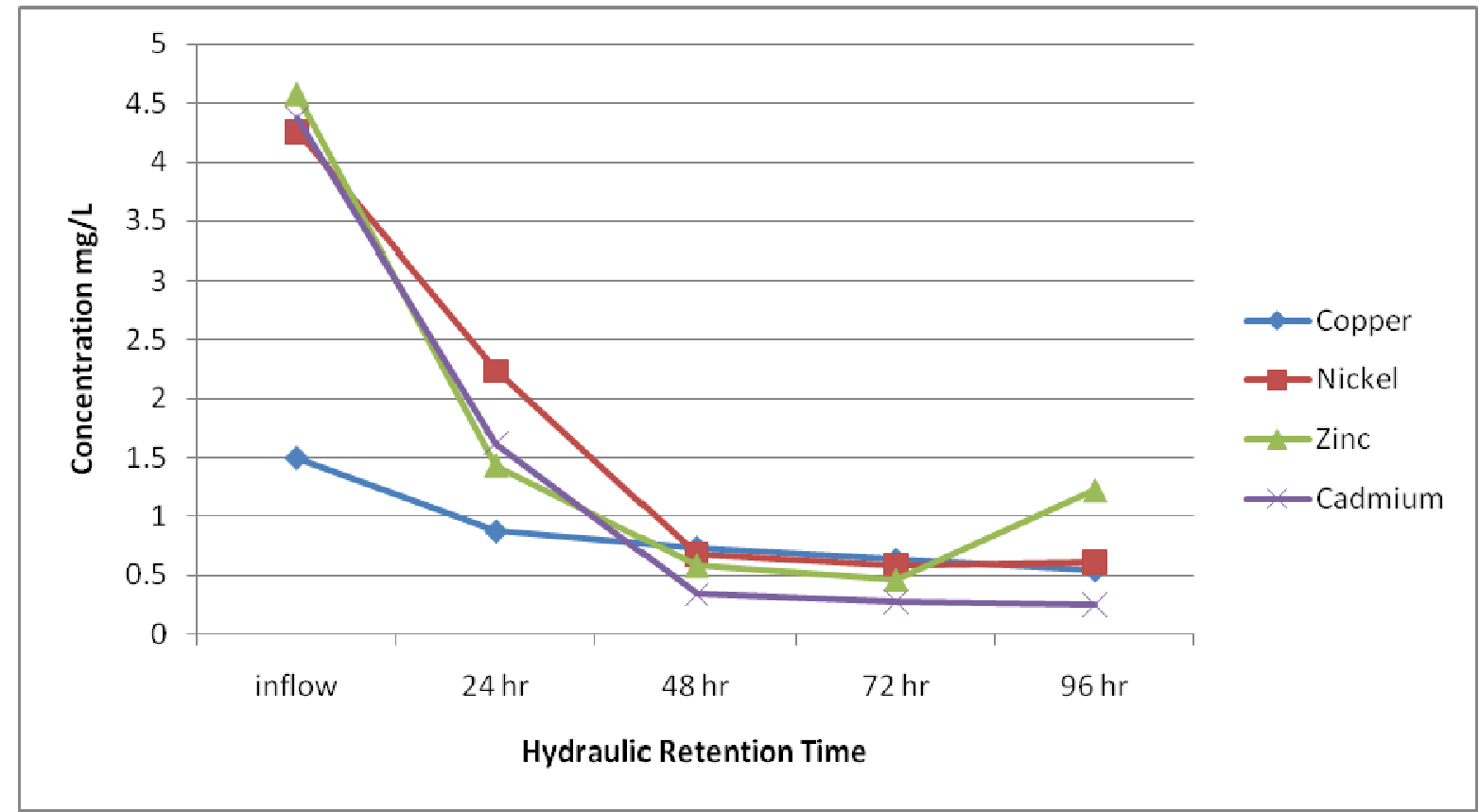

Figure 11. COC concentrations in series 1 during sampling period 3.

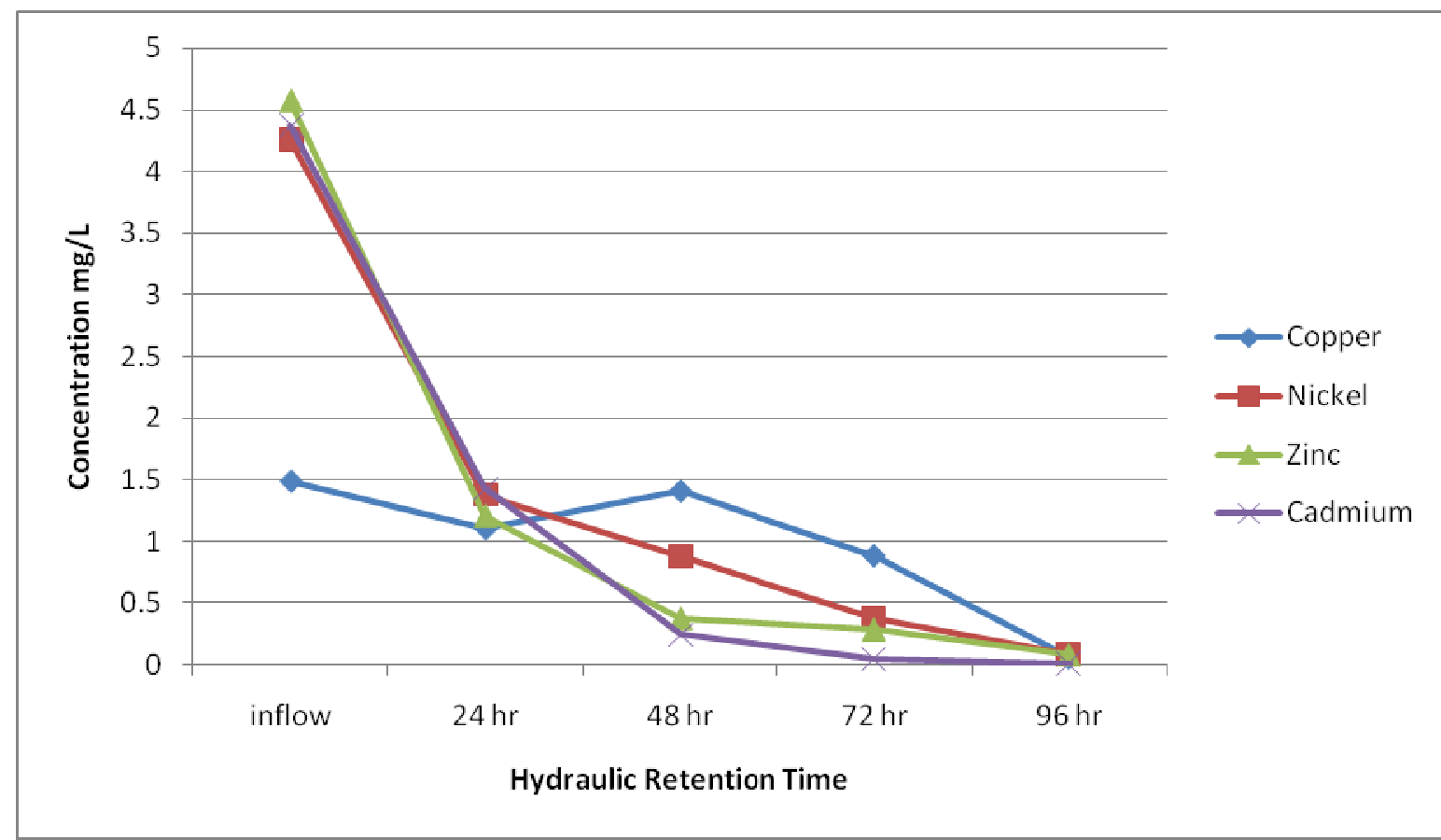

Figure 12. COC concentrations in series 2 during sampling period 3. 


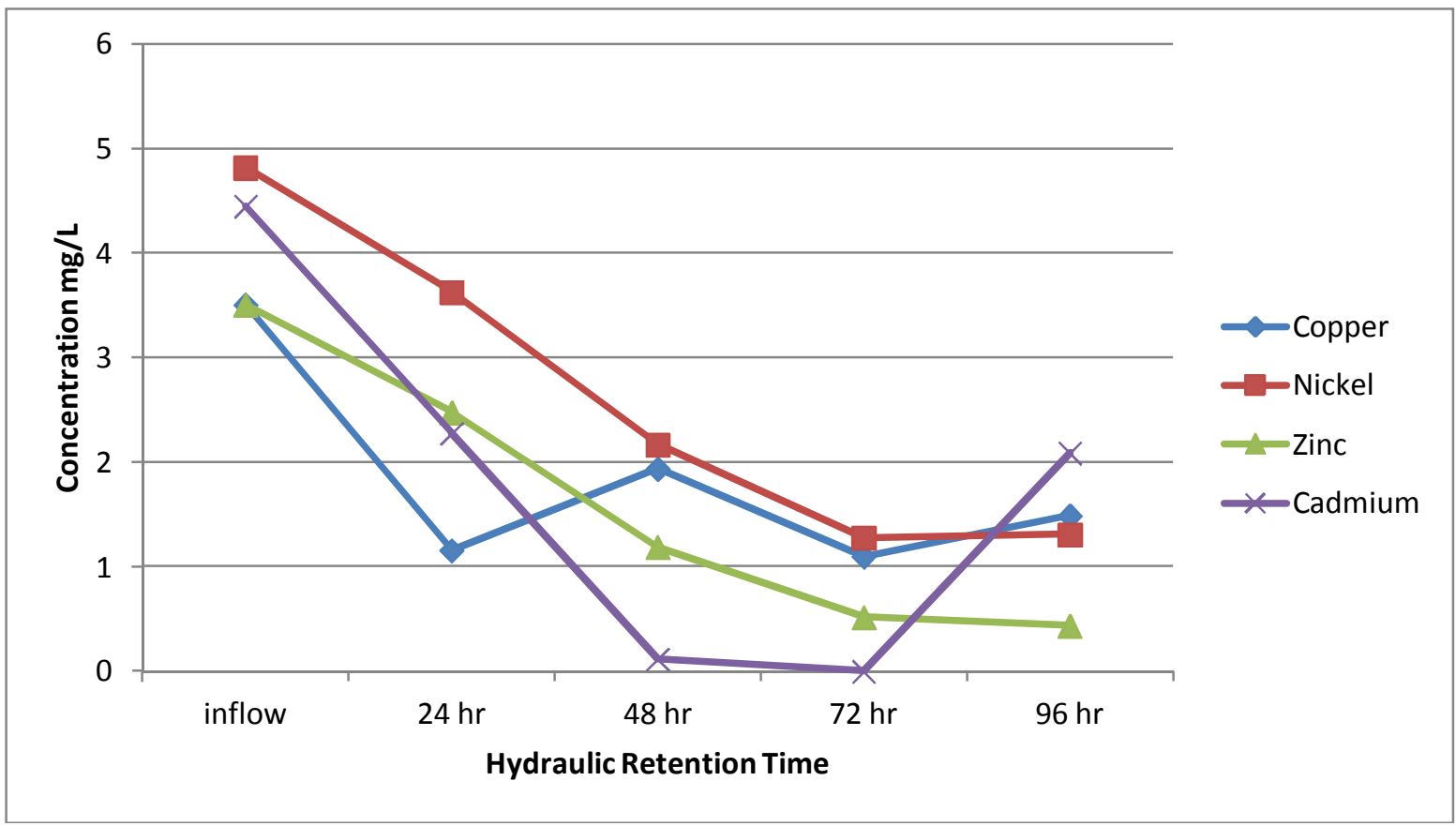

Figure 13. COC concentrations in series 1 during sampling period 4.

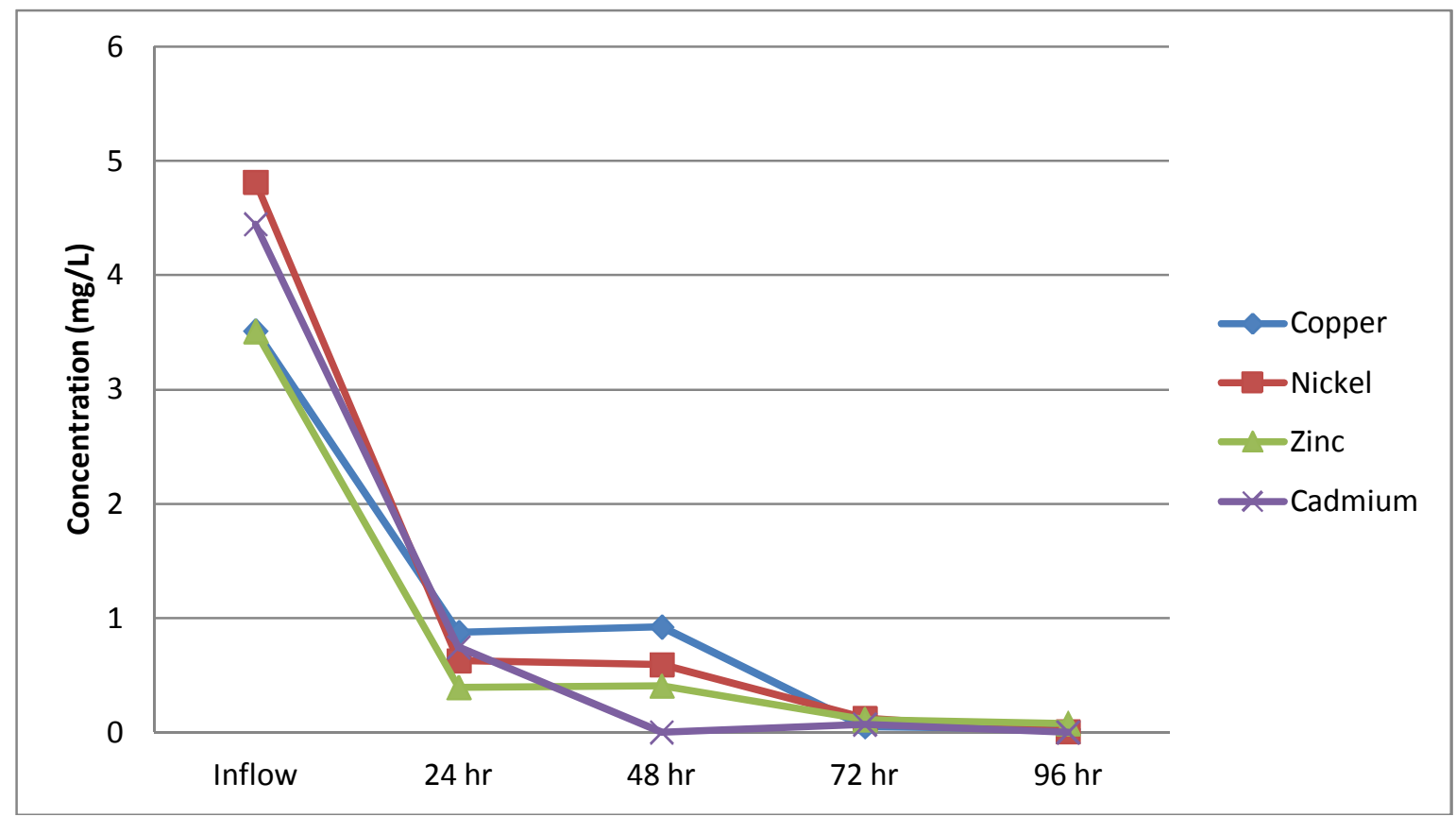

Figure 14. COC concentrations in series 2 during sampling period 4. 


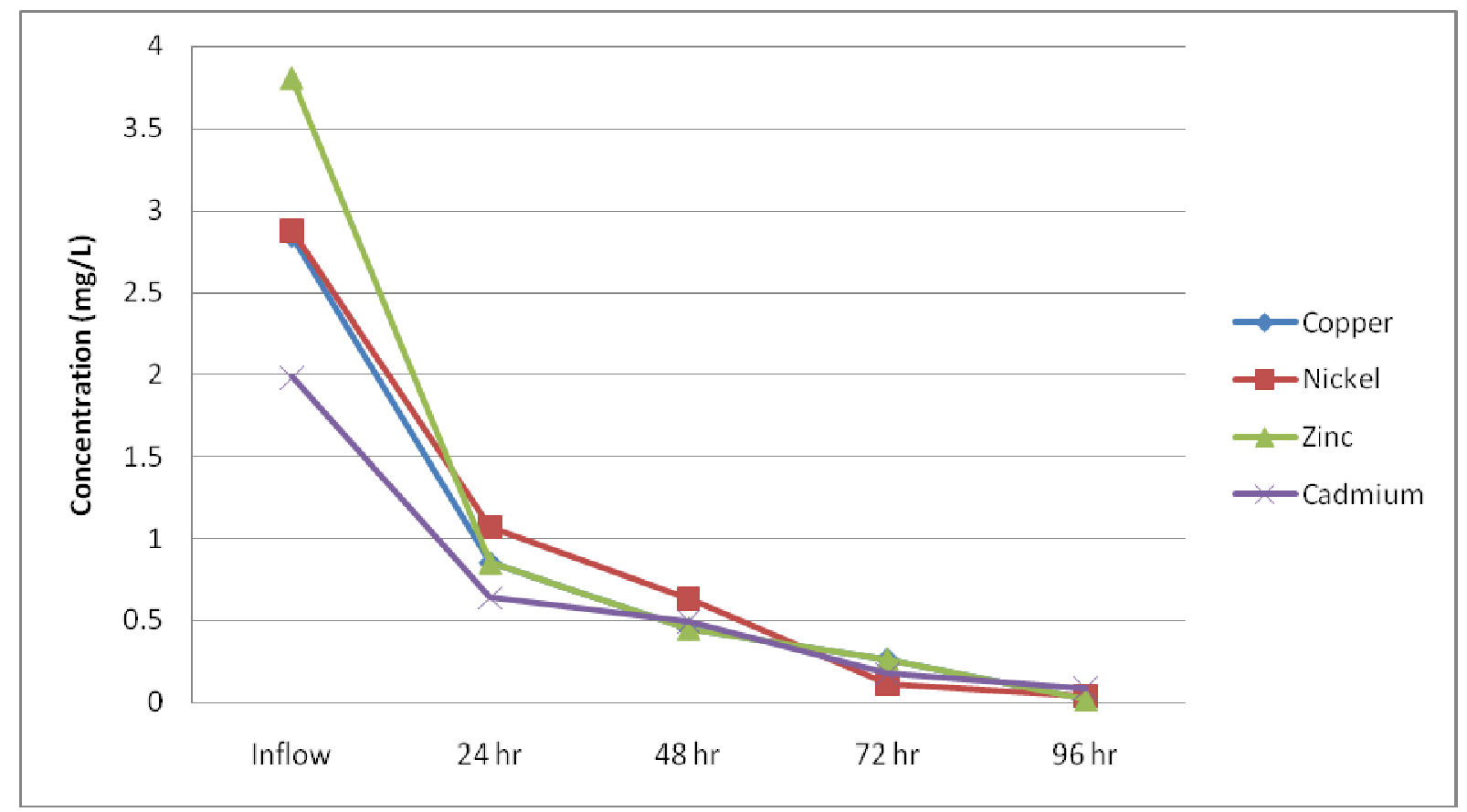

Figure 15. COC concentrations in series 1 during sampling period 5.

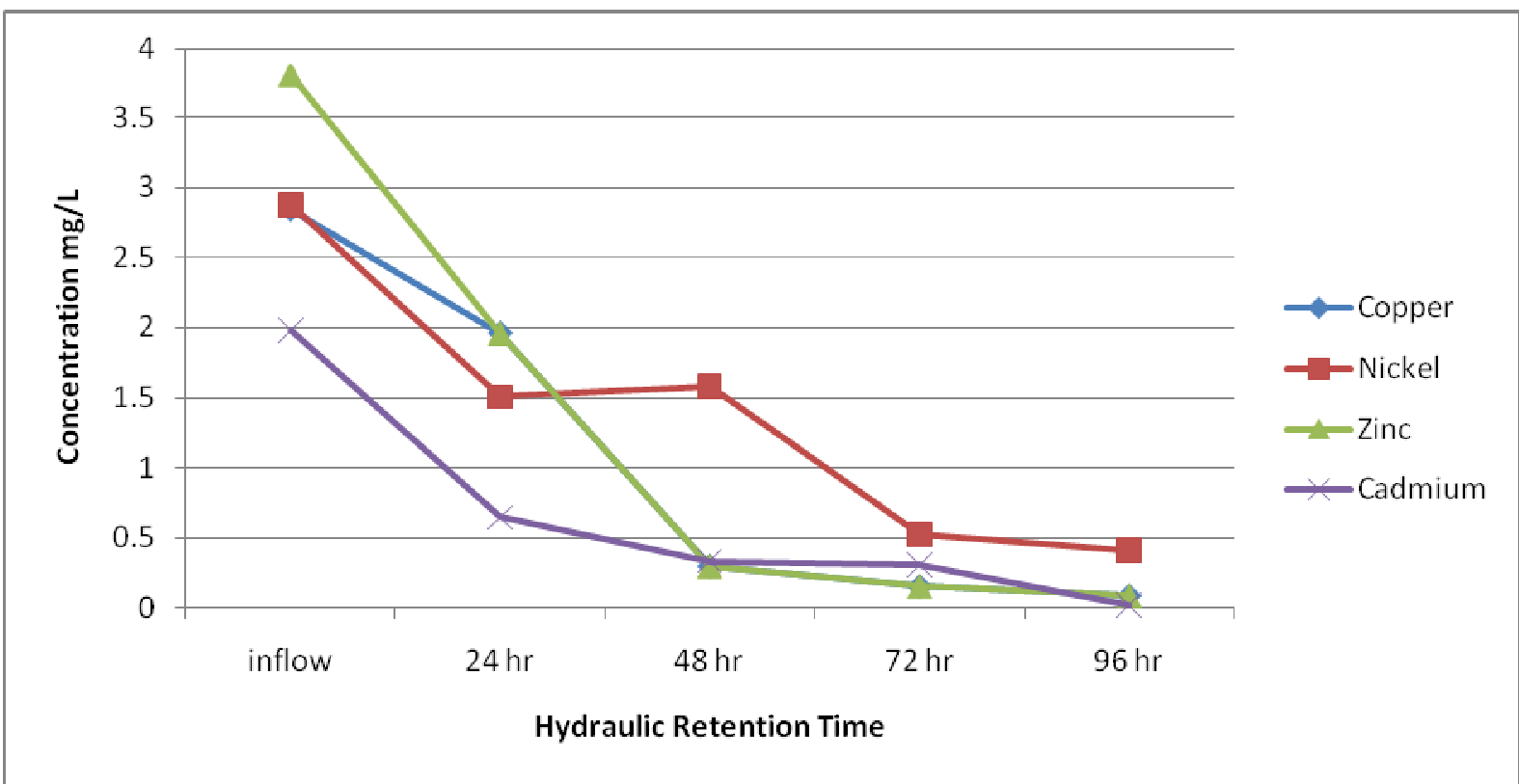

Figure 16. COC concentrations in series 2 during sampling period 5. 
Simulated PW containing metalloids (e.g. Se, As) and LMWOs was used for the pilot-scale experiment; simulating this water increases repeatability, allows easier manipulation of constituents and their respective concentrations, and is more cost effective than shipment of large volumes of PW for experimentation. After initial construction of the pilot-scale CWTS, a period of maturation was allowed, permitting the plants time to become established and initiate new growth. To accomplish acclimation, simulated PW was loaded into the pilot-scale CWTS at increasing COC concentrations for one month prior to addition of organic carbon sources; sucrose and nutrient (i.e. AquaSmart ${ }^{\mathrm{TM}}$ ) amendments were subsequently loaded into the pilotscale CWTS inflow for two weeks.

The primary objectives for this part of the project were to remove Se, As, and LMWOs from simulated PW. To measure performance (i.e. removal of COCs), simulated PW samples were collected from the pilot-scale CWTS inflow and outflow from each cell. Samples were analyzed for total Se, total As, and LMWOs, as well as general water chemistry parameters (i.e. explanatory parameters). Total Se was measured using atomic absorption spectrometry (hydride generation: Standard Method 3114 C; APHA 2005); total As was measured using graphite furnace atomic absorption spectrometry (modified EPA method 200.9). Total LMWOs were measured using a spectrofluorometric method modified from Zhou et al. (2009). Initial COC concentrations of Se, As and LMWOs in the simulated PW were $50 \mu \mathrm{g} / \mathrm{L}, 20 \mu \mathrm{g} / \mathrm{L}$, and $25 \mathrm{mg} / \mathrm{L}$, respectively; targeted outflow COC concentrations were $5 \mu \mathrm{g} \mathrm{Se} / \mathrm{L}, 5 \mu \mathrm{g}$ As/L, and $1 \mathrm{mg}$ LMWOs/L.

After the maturation and acclimation period, the goal of $5 \mu \mathrm{g} \mathrm{Se} / \mathrm{L}$ in the outflow water was achieved and maintained in the pilot-scale CWTS with the AquaSmart ${ }^{\mathrm{TM}}$ amendment (Figures 17-22). The sucrose amendment also achieved the goal of $5 \mu \mathrm{g} \mathrm{Se} / \mathrm{L}$, but with less consistency than the AquaSmart ${ }^{\mathrm{TM}}$ amendment. The untreated control series achieved no significant $\mathrm{Se}$ treatment. Arsenic treatment was accomplished, but less successfully than Se; As treatment is under further investigation. The goal of $5 \mu \mathrm{g}$ As/L in the outflow water was achieved by both the untreated control series and the AquaSmart ${ }^{\mathrm{TM}}$ series; however, removal was not achieved consistently (Figures 23-28). The goal of $1 \mathrm{mg}$ LMWOs/L in the outflow water was achieved consistently by all treatments, as well as the untreated control (Tables 39-44). This goal was achieved in the detention basin, prior to entering the pilot-scale CWTS. This implies that LMWOs brought to the surface in water produced from a well may readily volatilize when exposed to lower pressure than present in underground formations. 
Table 39. Se, As, LMWO analysis and explanatory parameters for Sampling Period 1

\begin{tabular}{|c|c|c|c|c|c|c|c|c|c|c|}
\hline \multirow[b]{2}{*}{ Sample } & \multicolumn{3}{|c|}{ Performance Parameters } & \multicolumn{7}{|c|}{ Explanatory Parameters } \\
\hline & $\begin{array}{c}{[\mathrm{Se}]} \\
(\mu \mathrm{g} / \mathrm{L})\end{array}$ & $\begin{array}{c}{[\mathrm{As}]} \\
(\mu \mathrm{g} / \mathrm{L})\end{array}$ & $\begin{array}{c}{[\mathrm{LMWO}]} \\
(\mathrm{mg} / \mathrm{L})\end{array}$ & $\begin{array}{c}\mathrm{DO} \\
(\mathrm{mg} / \mathrm{L})\end{array}$ & $\begin{array}{l}\text { Cond. } \\
(\mu \mathrm{S} / \mathrm{cm})\end{array}$ & $\begin{array}{c}\mathrm{pH} \\
\text { (S.U.) }\end{array}$ & $\begin{array}{c}\text { Redox } \\
(\mathrm{mV})\end{array}$ & $\begin{array}{l}\text { Temp } \\
\left({ }^{\circ} \mathrm{C}\right)\end{array}$ & $\begin{array}{l}\text { Alkalinity } \\
(\mathrm{mg} / \mathrm{L} \text { as } \\
\left.\mathrm{CaCO}_{3}\right) \\
\end{array}$ & $\begin{array}{l}\text { Hardness } \\
(\mathrm{mg} / \mathrm{L} \text { as } \\
\left.\mathrm{CaCO}_{3}\right) \\
\end{array}$ \\
\hline Inflow & 44.6 & 21.8 & bdl & 8.2 & 2812 & 7.35 & $\mathrm{~nm}$ & 25.8 & 40 & 138 \\
\hline Control 1 & 43.4 & 13.3 & bdl & 8.1 & 2838 & 7.00 & -203.9 & 25.1 & 24 & 134 \\
\hline Control 2 & 42.8 & 12.1 & bdl & 8.2 & 2856 & 6.90 & -181.0 & 24.3 & 24 & 134 \\
\hline Control 3 & 44.3 & 7.9 & bdl & 8.3 & 2868 & 6.80 & +10.4 & 24.1 & 22 & 132 \\
\hline Control 4 & 45.7 & 5.6 & bdl & 8.1 & 2880 & 6.73 & -115.4 & 24.0 & 24 & 136 \\
\hline AquaSmart $^{\mathrm{TM}} 1$ & 3.0 & 15.2 & bdl & 1.4 & 2845 & 6.56 & -126.8 & 25.2 & 38 & 140 \\
\hline AquaSmart $^{\mathrm{TM}} 2$ & 1.3 & 16.5 & bdl & 2.5 & 2871 & 6.67 & -69.9 & 24.0 & 34 & 144 \\
\hline AquaSmart $^{\mathrm{TM}} 3$ & bdl & 12.0 & bdl & 6.3 & 2890 & 6.95 & -45 & 23.2 & 74 & 148 \\
\hline AquaSmart $^{\mathrm{TM}} 4$ & bdl & 3.3 & bdl & 7.6 & 2930 & 7.05 & +54.2 & 23.0 & 70 & 148 \\
\hline Sucrose 1 & 18.3 & 19.2 & bdl & 5.1 & 2819 & 6.41 & -234.7 & 24.9 & 38 & 138 \\
\hline Sucrose 2 & 15.9 & 16.3 & bdl & 2.6 & 2802 & 6.38 & -97.6 & 24.0 & 46 & 136 \\
\hline Sucrose 3 & 9.9 & 15.0 & bdl & 4.8 & 2804 & 6.60 & -94.4 & 23.3 & 50 & 136 \\
\hline Sucrose 4 & 6.1 & 13.4 & bdl & 5.2 & 2812 & 6.63 & -44.3 & 23.2 & 64 & 146 \\
\hline
\end{tabular}

nm - not measured

bdl- below detection limit

Table 40. Se, As, LMWO analysis and explanatory parameters for Sampling Period 2

\begin{tabular}{|c|c|c|c|c|c|c|c|c|c|c|}
\hline \multirow[b]{2}{*}{ Sample } & \multicolumn{3}{|c|}{ Performance Parameters } & \multicolumn{7}{|c|}{ Explanatory Parameters } \\
\hline & $\begin{array}{c}{[\mathrm{Se}]} \\
(\mu \mathrm{g} / \mathrm{L})\end{array}$ & $\begin{array}{c}{[\mathrm{As}]} \\
(\mu \mathrm{g} / \mathrm{L})\end{array}$ & $\begin{array}{c}{[\mathrm{LMWO}]} \\
(\mathrm{mg} / \mathrm{L})\end{array}$ & $\begin{array}{c}\mathrm{DO} \\
(\mathrm{mg} / \mathrm{L})\end{array}$ & $\begin{array}{l}\text { Cond. } \\
(\mu \mathrm{S} / \mathrm{cm})\end{array}$ & $\begin{array}{c}\mathrm{pH} \\
\text { (S.U.) }\end{array}$ & $\begin{array}{c}\text { Redox } \\
(\mathrm{mV})\end{array}$ & $\begin{array}{l}\text { Temp } \\
\left({ }^{\circ} \mathrm{C}\right)\end{array}$ & $\begin{array}{c}\text { Alkalinity } \\
(\mathrm{mg} / \mathrm{L} \\
\left.\mathrm{CaCO}_{3}\right)\end{array}$ & $\begin{array}{c}\text { Hardness } \\
(\mathrm{mg} / \mathrm{L} \\
\left.\mathrm{CaCO}_{3}\right)\end{array}$ \\
\hline Inflow & 49.7 & 14.0 & bdl & 8.4 & 2612 & 7.26 & $\mathrm{~nm}$ & 13.2 & 42 & 140 \\
\hline Control 1 & 45.1 & 10.6 & bdl & 8.2 & 2630 & 7.06 & -208.6 & 15.1 & 26 & 138 \\
\hline Control 2 & 46.3 & 7.5 & bdl & 8.1 & 2643 & 6.86 & -188.2 & 15.9 & 26 & 136 \\
\hline Control 3 & 43.7 & 6.4 & bdl & 8.2 & 2666 & 6.88 & +9.22 & 16.5 & 24 & 130 \\
\hline Control 4 & 44.6 & 5.0 & bdl & 8.2 & 2701 & 6.80 & -125.2 & 16.9 & 28 & 132 \\
\hline AquaSmart $^{\mathrm{TM}} 1$ & 8.8 & 9.5 & bdl & 1.2 & 2642 & 6.49 & -135.2 & 14.9 & 40 & 142 \\
\hline AquaSmart $^{\mathrm{TM}} 2$ & 2.8 & 6.9 & bdl & 2.3 & 2665 & 6.64 & -84.6 & 15.7 & 38 & 142 \\
\hline AquaSmart $^{\mathrm{TM}} 3$ & 1.1 & 7.8 & bdl & 5.7 & 2684 & 6.94 & -51.3 & 16.7 & 76 & 152 \\
\hline AquaSmart ${ }^{\mathrm{TM}} 4$ & bdl & 6.3 & bdl & 7.4 & 2713 & 7.10 & +46.5 & 16.5 & 74 & 154 \\
\hline Sucrose 1 & 38.2 & 8.2 & bdl & 4.3 & 2680 & 6.34 & -241.2 & 15.2 & 42 & 140 \\
\hline Sucrose 2 & 24.2 & 5.2 & bdl & 2.2 & 2695 & 6.54 & -101.3 & 15.8 & 48 & 140 \\
\hline Sucrose 3 & 13.8 & 5.1 & bdl & 4.5 & 2721 & 6.72 & -91.2 & 16.3 & 50 & 138 \\
\hline Sucrose 4 & 8.1 & 5.5 & bdl & 5.4 & 2732 & 6.74 & -49.2 & 16.9 & 66 & 152 \\
\hline
\end{tabular}

nm - not measured

bdl - below detection limit 
Table 41. Se, As, LMWO analysis and explanatory parameters for Sampling Period 3

\begin{tabular}{|c|c|c|c|c|c|c|c|c|c|c|}
\hline \multirow[b]{2}{*}{ Sample } & \multicolumn{3}{|c|}{ Performance Parameters } & \multicolumn{7}{|c|}{ Explanatory Parameters } \\
\hline & $\begin{array}{c}{[\mathrm{Se}]} \\
(\mu \mathrm{g} / \mathrm{L})\end{array}$ & $\begin{array}{c}{[\mathrm{As}]} \\
(\mu \mathrm{g} / \mathrm{L})\end{array}$ & $\begin{array}{c}{[\mathrm{LMWO}]} \\
(\mathrm{mg} / \mathrm{L})\end{array}$ & $\begin{array}{c}\mathrm{DO} \\
(\mathrm{mg} / \mathrm{L})\end{array}$ & $\begin{array}{c}\text { Cond. } \\
(\mu \mathrm{S} / \mathrm{cm})\end{array}$ & $\begin{array}{c}\mathrm{pH} \\
\text { (S.U.) }\end{array}$ & $\begin{array}{c}\text { Redox } \\
(\mathrm{mV})\end{array}$ & $\begin{array}{l}\text { Temp } \\
\left({ }^{\circ} \mathrm{C}\right)\end{array}$ & $\begin{array}{c}\text { Alkalinity } \\
(\mathrm{mg} / \mathrm{L} \\
\left.\mathrm{CaCO}_{3}\right)\end{array}$ & $\begin{array}{c}\text { Hardness } \\
(\mathrm{mg} / \mathrm{L} \\
\left.\mathrm{CaCO}_{3}\right)\end{array}$ \\
\hline Inflow & 46.5 & 18.4 & bdl & 8.5 & 1727 & 7.15 & $\mathrm{~nm}$ & 11.5 & 40 & 136 \\
\hline Control 1 & 44.9 & 16.5 & bdl & 8.3 & 1865 & 6.89 & -194.9 & 13.0 & 28 & 132 \\
\hline Control 2 & 41.9 & 13.1 & bdl & 8.0 & 2086 & 6.71 & -133.0 & 14.8 & 24 & 132 \\
\hline Control 3 & 39.1 & 11.9 & bdl & 8.2 & 2145 & 6.59 & +100.4 & 15.0 & 26 & 128 \\
\hline Control 4 & 37.9 & 10.9 & bdl & 8.1 & 2128 & 6.62 & +111.6 & 14.6 & 26 & 128 \\
\hline AquaSmart ${ }^{\mathrm{TM}} 1$ & 7.9 & 19.0 & bdl & 1.1 & 1849 & 6.43 & -112.2 & 13.5 & 42 & 140 \\
\hline AquaSmart ${ }^{\mathrm{TM}} 2$ & 2.2 & 15.3 & bdl & 2.1 & 1908 & 6.54 & -46.2 & 14.7 & 36 & 140 \\
\hline AquaSmart $^{\mathrm{TM}} 3$ & bdl & 15.6 & bdl & 5.2 & 2055 & 6.87 & -36.3 & 13.7 & 74 & 148 \\
\hline AquaSmart ${ }^{\mathrm{TM}} 4$ & bdl & 14.7 & bdl & 7.7 & 2020 & 6.90 & +68.4 & 13.5 & 76 & 152 \\
\hline Sucrose 1 & 36.2 & 16.7 & bdl & 3.9 & 1805 & 6.21 & -221.7 & 13.0 & 40 & 138 \\
\hline Sucrose 2 & 26.5 & 17.1 & bdl & 2.0 & 1867 & 6.43 & -63.5 & 13.7 & 46 & 136 \\
\hline Sucrose 3 & 13.8 & 17.2 & bdl & 4.3 & 2055 & 6.75 & -46.3 & 14.2 & 48 & 140 \\
\hline Sucrose 4 & 7.3 & 18.3 & bdl & 5.1 & 2020 & 6.73 & -16.3 & 13.6 & 58 & 148 \\
\hline
\end{tabular}

$\mathrm{nm}-$ not measured

bdl - below detection limit

Table 42. Se, As, LMWO analysis and explanatory parameters for Sampling Period 4

\begin{tabular}{|c|c|c|c|c|c|c|c|c|c|c|}
\hline \multirow[b]{2}{*}{ Sample } & \multicolumn{3}{|c|}{ Performance Parameters } & \multicolumn{7}{|c|}{ Explanatory Parameters } \\
\hline & $\begin{array}{c}{[\mathrm{Se}]} \\
(\mu \mathrm{g} / \mathrm{L})\end{array}$ & $\begin{array}{c}{[\mathrm{As}]} \\
(\mu \mathrm{g} / \mathrm{L})\end{array}$ & $\begin{array}{c}{[\mathrm{LMWO}]} \\
(\mathrm{mg} / \mathrm{L})\end{array}$ & $\begin{array}{c}\mathrm{DO} \\
(\mathrm{mg} / \mathrm{L})\end{array}$ & $\begin{array}{l}\text { Cond. } \\
(\mu \mathrm{S} / \mathrm{cm})\end{array}$ & $\begin{array}{c}\mathrm{pH} \\
\text { (S.U.) }\end{array}$ & $\begin{array}{l}\text { Redox } \\
(\mathrm{mV})\end{array}$ & $\begin{array}{c}\text { Temp } \\
\left({ }^{\circ} \mathrm{C}\right)\end{array}$ & $\begin{array}{c}\text { Alkalinity } \\
(\mathrm{mg} / \mathrm{L} \\
\left.\mathrm{CaCO}_{3}\right)\end{array}$ & $\begin{array}{c}\text { Hardness } \\
\left(\mathrm{mg}_{\mathrm{L}} \mathrm{L}\right. \\
\left.\mathrm{CaCO}_{3}\right)\end{array}$ \\
\hline Inflow & 42.7 & 18.4 & bdl & 8.4 & 2103 & 7.26 & $\mathrm{~nm}$ & 14.6 & 44 & 144 \\
\hline Control 1 & 38.3 & 13.5 & bdl & 8.1 & 2154 & 6.94 & -168.5 & 15.4 & 34 & 136 \\
\hline Control 2 & 45.7 & 10.4 & bdl & 8.2 & 2209 & 6.76 & -115.2 & 16.8 & 28 & 134 \\
\hline Control 3 & 39.7 & 8 & bdl & 8.0 & 2231 & 6.62 & +64.2 & 17.2 & 30 & 130 \\
\hline Control 4 & 39.7 & 6 & bdl & 8.1 & 2281 & 6.54 & +80.5 & 17.8 & 28 & 126 \\
\hline AquaSmart $^{\mathrm{TM}} 1$ & 27.3 & 15.1 & bdl & 0.9 & 2145 & 6.52 & -145.6 & 15.2 & 44 & 146 \\
\hline AquaSmart $^{\mathrm{TM}} 2$ & 4.5 & 8.1 & bdl & 1.6 & 2201 & 6.59 & -64.2 & 16.9 & 38 & 144 \\
\hline AquaSmart $^{\mathrm{TM}} 3$ & bdl & 8.6 & bdl & 4.6 & 2263 & 6.90 & -29.5 & 17.6 & 72 & 146 \\
\hline AquaSmart ${ }^{\mathrm{TM}} 4$ & bdl & 9 & bdl & 7.4 & 2298 & 6.87 & +58.6 & 17.9 & 78 & 150 \\
\hline Sucrose 1 & 29.8 & 18.4 & bdl & 1.7 & 2142 & 6.42 & -240.3 & 15.6 & 42 & 140 \\
\hline Sucrose 2 & 3.0 & 11.6 & bdl & 1.6 & 2211 & 6.51 & -52.3 & 16.4 & 44 & 142 \\
\hline Sucrose 3 & 6.5 & 8.2 & bdl & 4.1 & 2245 & 6.65 & -50.1 & 17.5 & 46 & 144 \\
\hline Sucrose 4 & bdl & 6.3 & bdl & 4.4 & 2284 & 6.64 & -25.9 & 18.0 & 60 & 150 \\
\hline
\end{tabular}

nm - not measured

bdl - below detection limit 
Table 43. Se, As, LMWO analysis and explanatory parameters for Sampling Period 5

\begin{tabular}{|c|c|c|c|c|c|c|c|c|c|c|}
\hline \multirow[b]{2}{*}{ Sample } & \multicolumn{3}{|c|}{ Performance Parameters } & \multicolumn{7}{|c|}{ Explanatory Parameters } \\
\hline & $\begin{array}{c}{[\mathrm{Se}]} \\
(\mu \mathrm{g} / \mathrm{L}) \\
\end{array}$ & $\begin{array}{c}{[\mathrm{As}]} \\
(\mu \mathrm{g} / \mathrm{L})\end{array}$ & $\begin{array}{c}{[\mathrm{LMWO}]} \\
(\mathrm{mg} / \mathrm{L})\end{array}$ & $\begin{array}{c}\mathrm{DO} \\
(\mathrm{mg} / \mathrm{L})\end{array}$ & $\begin{array}{c}\text { Cond. } \\
(\mu \mathrm{S} / \mathrm{cm})\end{array}$ & $\begin{array}{c}\mathrm{pH} \\
\text { (S.U.) } \\
\end{array}$ & $\begin{array}{c}\text { Redox } \\
(\mathrm{mV})\end{array}$ & $\begin{array}{c}\text { Temp } \\
\left({ }^{\circ} \mathrm{C}\right)\end{array}$ & $\begin{array}{c}\text { Alkalinity } \\
(\mathrm{mg} / \mathrm{L} \\
\left.\mathrm{CaCO}_{3}\right) \\
\end{array}$ & $\begin{array}{c}\text { Hardness } \\
(\mathrm{mg} / \mathrm{L} \\
\left.\mathrm{CaCO}_{3}\right)\end{array}$ \\
\hline Inflow & 76.0 & 18.3 & bdl & 8.2 & 2210 & 7.52 & $\mathrm{~nm}$ & 15.9 & 44 & 146 \\
\hline Control 1 & 52.7 & 14.1 & bdl & 8.0 & 2264 & 6.89 & -171.2 & 16.2 & 32 & 132 \\
\hline Control 2 & 55.2 & 8.7 & bdl & 8.0 & 2300 & 6.74 & -119.4 & 16.7 & 32 & 136 \\
\hline Control 3 & 56.7 & 7.1 & bdl & 7.8 & 2323 & 6.64 & +52.3 & 17.0 & 32 & 134 \\
\hline Control 4 & 58.2 & 4.3 & bdl & 7.9 & 2384 & 6.41 & +65.3 & 17.5 & 30 & 128 \\
\hline AquaSmart $^{\mathrm{TM}} 1$ & 11.2 & 17.9 & bdl & 1.1 & 2225 & 6.58 & -154.8 & 16.3 & 46 & 152 \\
\hline AquaSmart $^{\mathrm{TM}} 2$ & bdl & 9.8 & bdl & 1.7 & 2284 & 6.69 & -71.6 & 16.9 & 36 & 148 \\
\hline AquaSmart ${ }^{\mathrm{TM}} 3$ & bdl & 8.7 & bdl & 5.2 & 2317 & 6.87 & -35.2 & 17.4 & 68 & 154 \\
\hline AquaSmart ${ }^{\mathrm{TM}} 4$ & bdl & 9.7 & bdl & 7.5 & 2345 & 6.96 & +54.3 & 17.6 & 76 & 156 \\
\hline Sucrose 1 & 6.3 & 7.1 & bdl & 1.9 & 2231 & 6.25 & -234.5 & 16.5 & 40 & 142 \\
\hline Sucrose 2 & 2.5 & 5.4 & bdl & 2.0 & 2256 & 6.49 & -64.2 & 16.7 & 46 & 144 \\
\hline Sucrose 3 & 2.2 & 5.4 & bdl & 4.0 & 2289 & 6.54 & -42.1 & 17.0 & 42 & 138 \\
\hline Sucrose 4 & 5.8 & 3.5 & bdl & 4.3 & 2321 & 6.59 & -34.8 & 17.5 & 54 & 146 \\
\hline
\end{tabular}

nm - not measured

bdl - below detection limit

Table 44. Se, As, LMWO analysis and explanatory parameters for Sampling Period 6

\begin{tabular}{|c|c|c|c|c|c|c|c|c|c|c|}
\hline \multirow[b]{2}{*}{ Sample } & \multicolumn{3}{|c|}{ Performance Parameters } & \multicolumn{7}{|c|}{ Explanatory Parameters } \\
\hline & $\begin{array}{c}{[\mathrm{Se}]} \\
(\mu \mathrm{g} / \mathrm{L}) \\
\end{array}$ & $\begin{array}{c}{[\mathrm{As}]} \\
(\mu \mathrm{g} / \mathrm{L}) \\
\end{array}$ & $\begin{array}{c}{[\mathrm{LMWO}]} \\
(\mathrm{mg} / \mathrm{L})\end{array}$ & $\begin{array}{c}\mathrm{DO} \\
(\mathrm{mg} / \mathrm{L}) \\
\end{array}$ & $\begin{array}{l}\text { Cond. } \\
(\mu \mathrm{S} / \mathrm{cm})\end{array}$ & $\begin{array}{c}\mathrm{pH} \\
\text { (S.U.) } \\
\end{array}$ & $\begin{array}{c}\text { Redox } \\
(\mathrm{mV})\end{array}$ & $\begin{array}{c}\text { Temp } \\
\left({ }^{\circ} \mathrm{C}\right)\end{array}$ & $\begin{array}{c}\text { Alkalinity } \\
(\mathrm{mg} / \mathrm{L} \\
\left.\mathrm{CaCO}_{3}\right) \\
\end{array}$ & $\begin{array}{c}\text { Hardness } \\
(\mathrm{mg} / \mathrm{L} \\
\left.\mathrm{CaCO}_{3}\right)\end{array}$ \\
\hline Inflow & 50.8 & 17.2 & bdl & 8.6 & 2525 & 7.4 & $\mathrm{~nm}$ & 16.3 & 42 & 144 \\
\hline Control 1 & 51.6 & 22.6 & bdl & 8.4 & 2528 & 6.91 & -170.3 & 16.8 & 34 & 134 \\
\hline Control 2 & 61.4 & 4.4 & bdl & 8.9 & 2500 & 6.93 & -120.4 & 17.1 & 32 & 134 \\
\hline Control 3 & 63.6 & 4.0 & bdl & 8.4 & 2523 & 6.96 & +51.8 & 17.4 & 34 & 136 \\
\hline Control 4 & 56.4 & 2.4 & bdl & 7.8 & 2489 & 6.92 & +65.4 & 17.8 & 32 & 130 \\
\hline AquaSmart $^{\mathrm{TM}} 1$ & 6.3 & 12.7 & bdl & 3.5 & 2345 & 6.60 & -157.5 & 16.7 & 48 & 152 \\
\hline AquaSmart $^{\mathrm{TM}} 2$ & 5.0 & 15.1 & bdl & 4.8 & 2360 & 6.82 & -72.6 & 17.0 & 38 & 150 \\
\hline AquaSmart $^{\mathrm{TM}} 3$ & 1.9 & 10.1 & bdl & 6.1 & 2230 & 6.82 & -38.7 & 17.5 & 70 & 156 \\
\hline AquaSmart $^{\mathrm{TM}} 4$ & bdl & 6.9 & bdl & 6.8 & 2290 & 6.79 & +52.6 & 17.8 & 78 & 158 \\
\hline Sucrose 1 & 22.2 & 13.5 & bdl & 5.6 & 2330 & 6.32 & -245.1 & 16.3 & 42 & 144 \\
\hline Sucrose 2 & 11.7 & 10.5 & bdl & 2.4 & 2280 & 6.51 & -68.2 & 16.7 & 48 & 146 \\
\hline Sucrose 3 & 9.2 & 9.5 & bdl & 5.0 & 2260 & 6.68 & -45.6 & 17.2 & 44 & 140 \\
\hline Sucrose 4 & 2.9 & 9.8 & bdl & 4.9 & 2265 & 6.71 & -35.6 & 17.7 & 56 & 148 \\
\hline
\end{tabular}

nm - not measured

bdl - below detection limit 


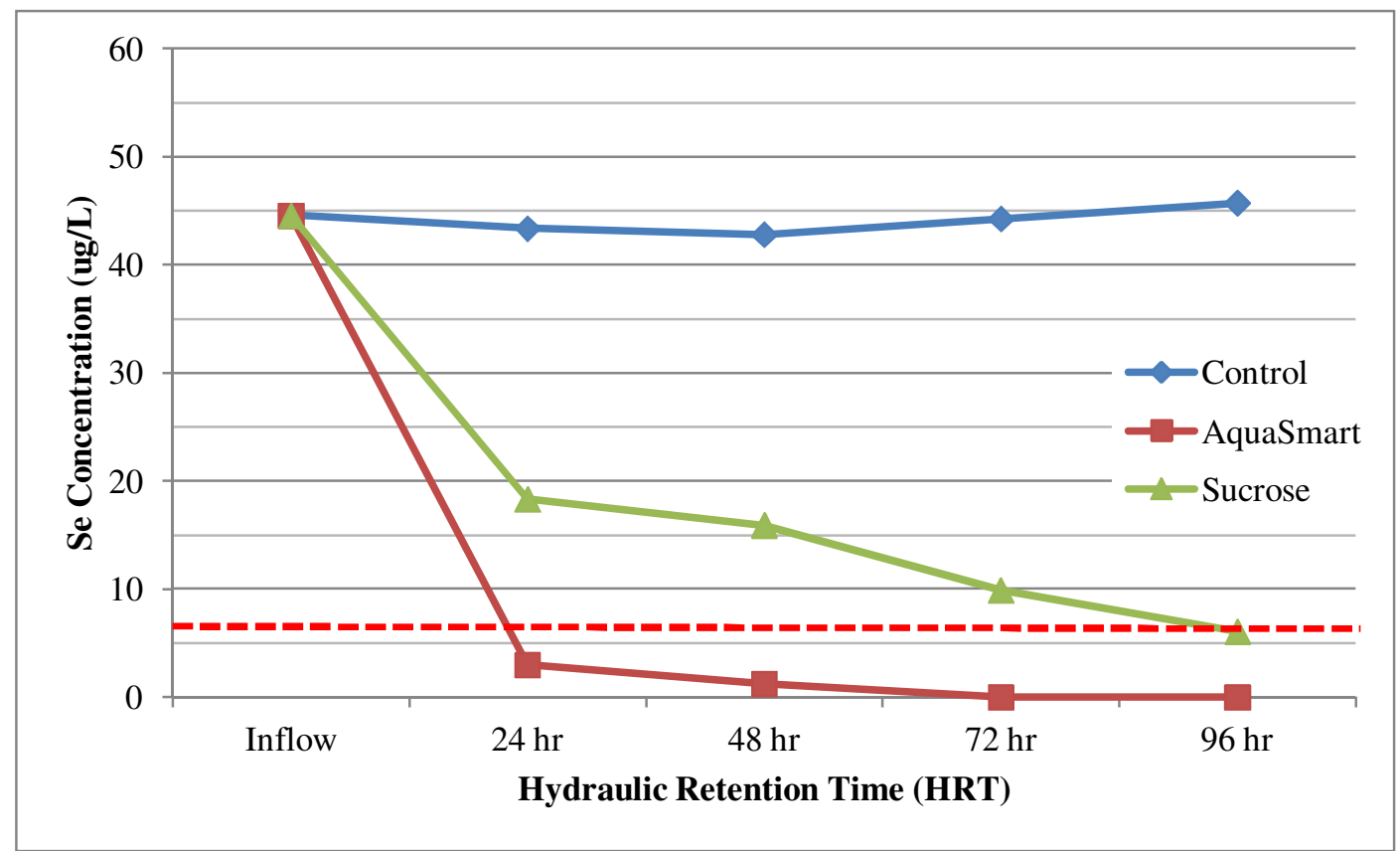

Figure 17. Se concentration during sampling period 1 with a goal of $5 \mu \mathrm{g} \mathrm{Se} / \mathrm{L}$ (red dashed line)

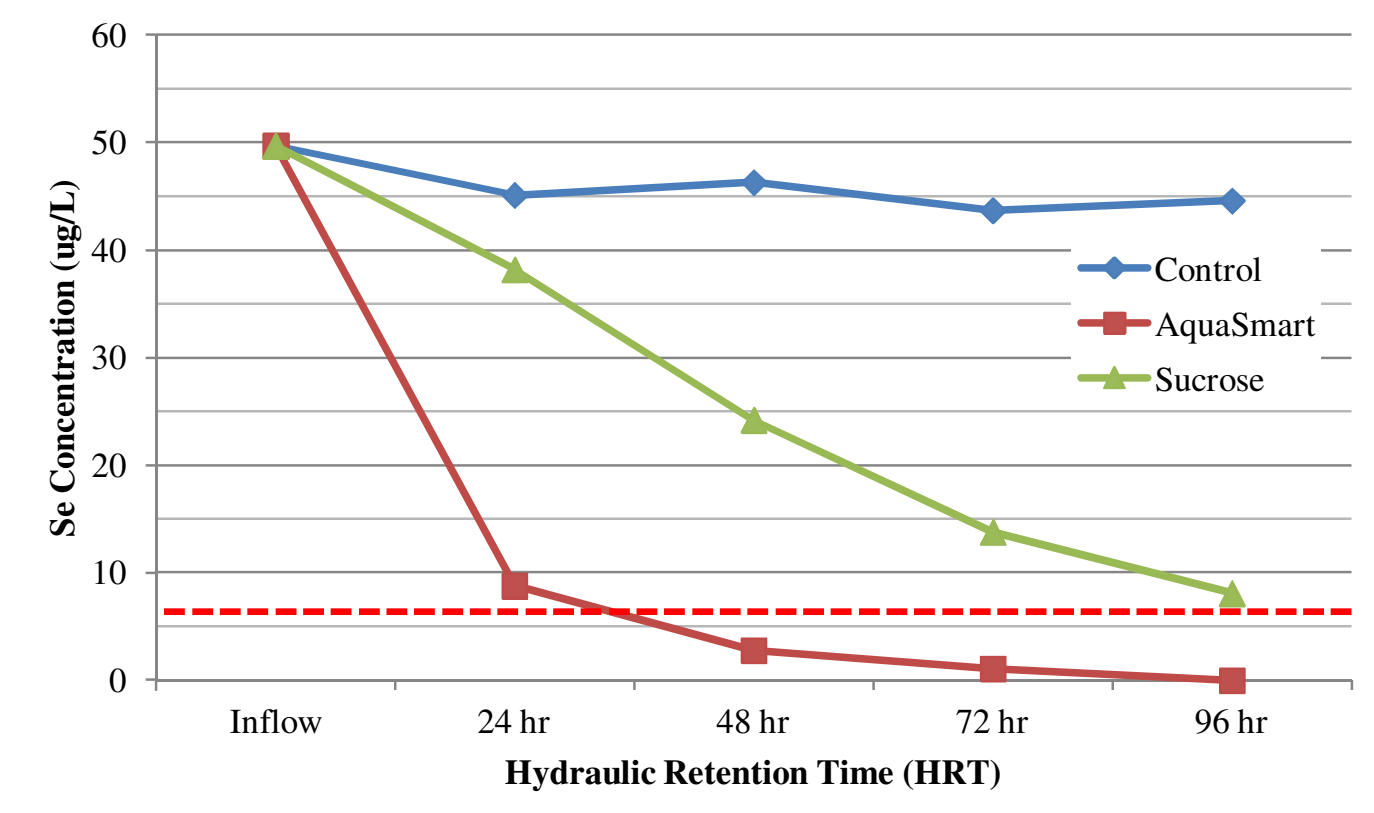

Figure 18. Se concentration during sampling period 2 with a goal of $5 \mu \mathrm{g} \mathrm{Se} / \mathrm{L}$ (red dashed line) 


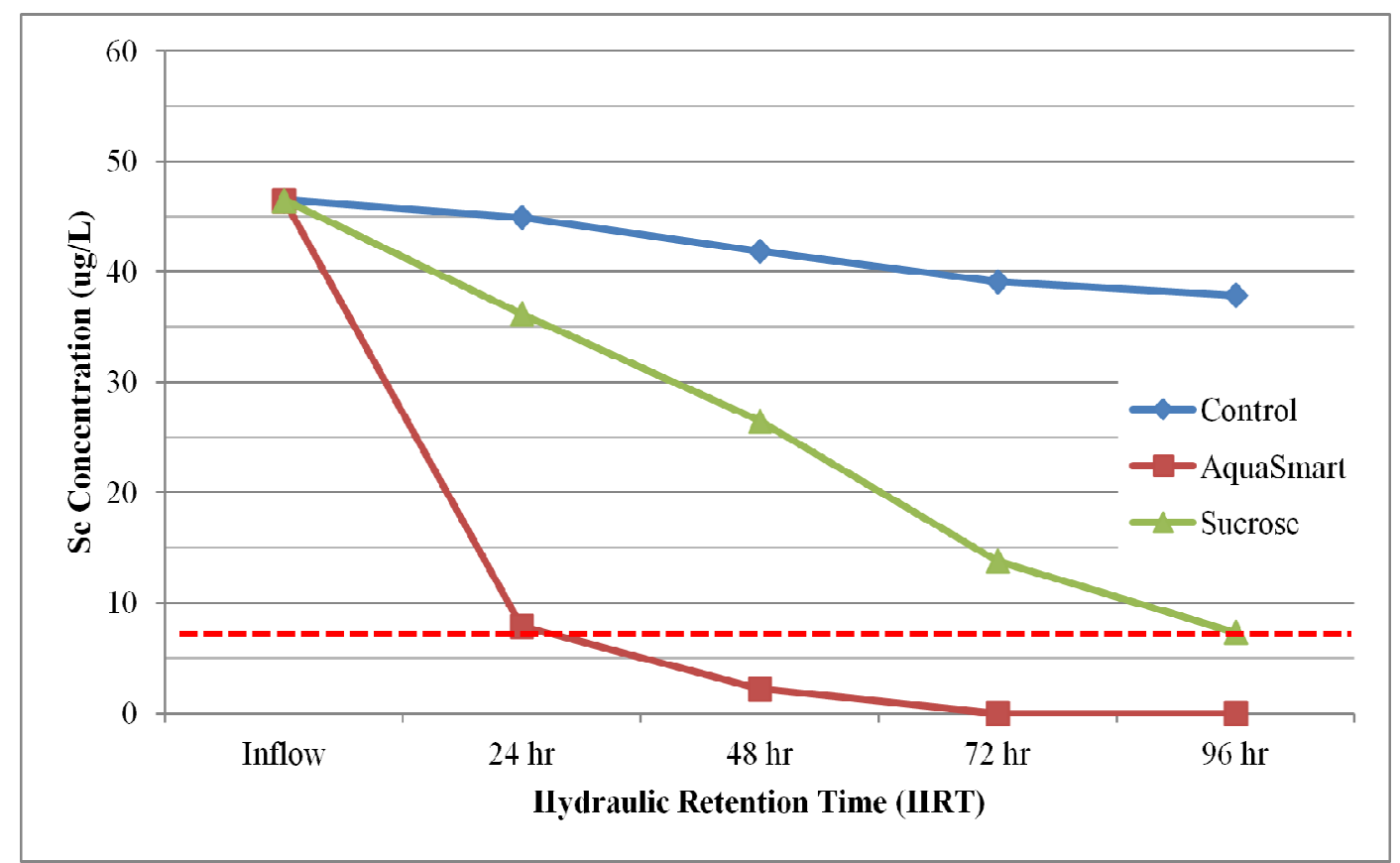

Figure 19. Se concentration during sampling period 3 with a goal of $5 \mu \mathrm{g} \mathrm{Se} / \mathrm{L}$ (red dashed line)

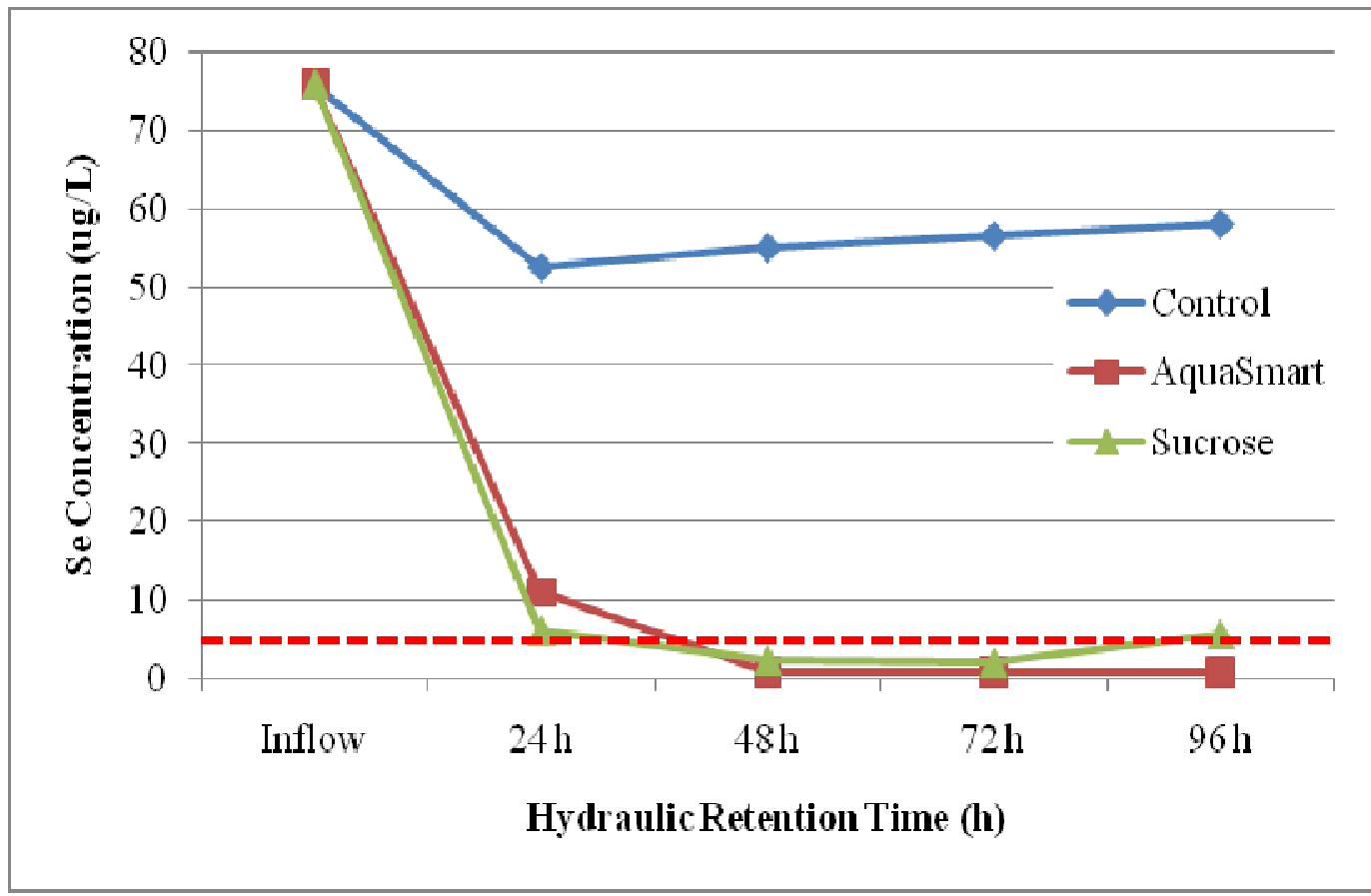

Figure 20. Se concentration during sampling period 4 with a goal of $5 \mu \mathrm{g} \mathrm{Se} / \mathrm{L}$ (red dashed line) 


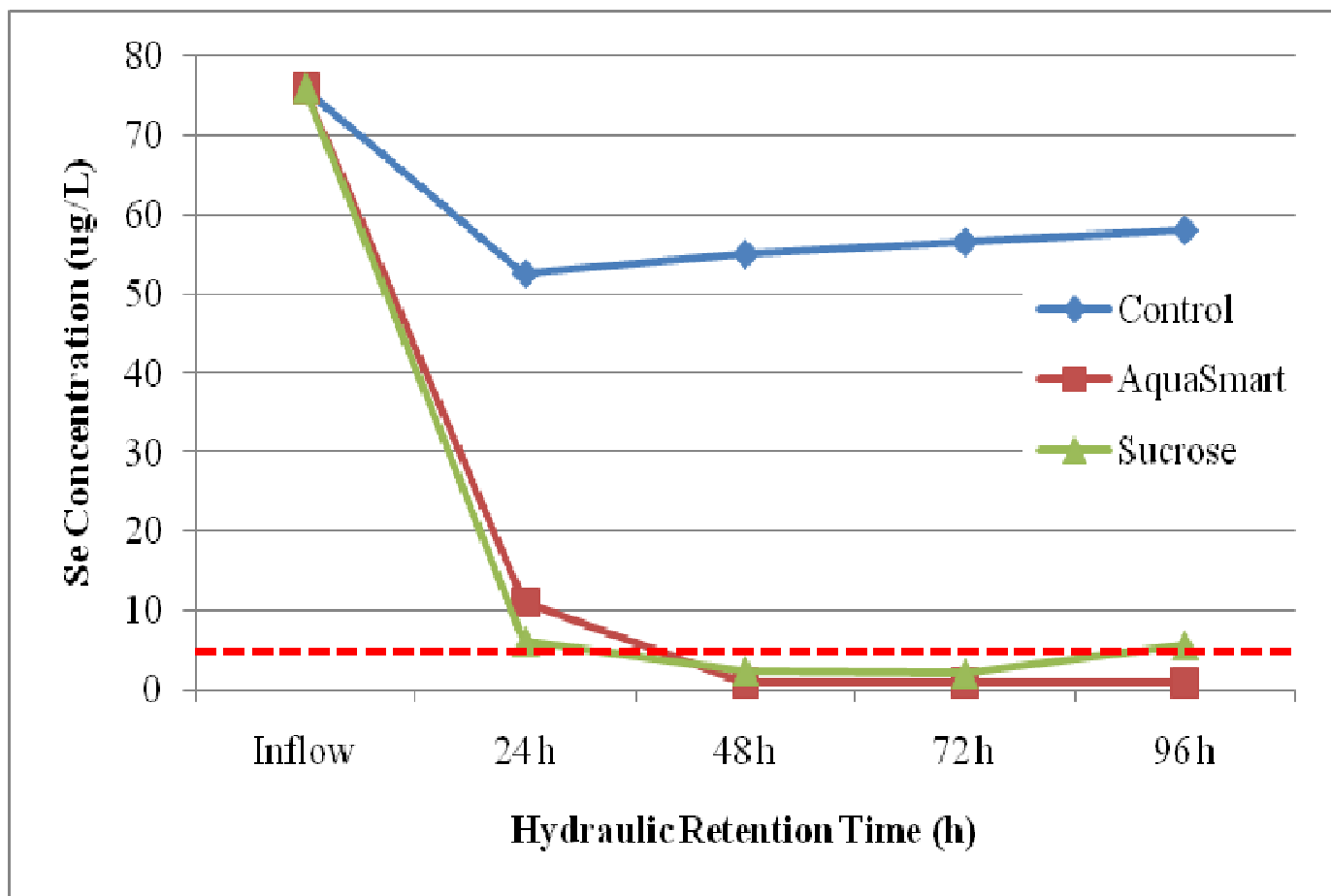

Figure 21. Se concentration during sampling period 5 with a goal of $5 \mu \mathrm{g} \mathrm{Se} / \mathrm{L}$ (red dashed line)

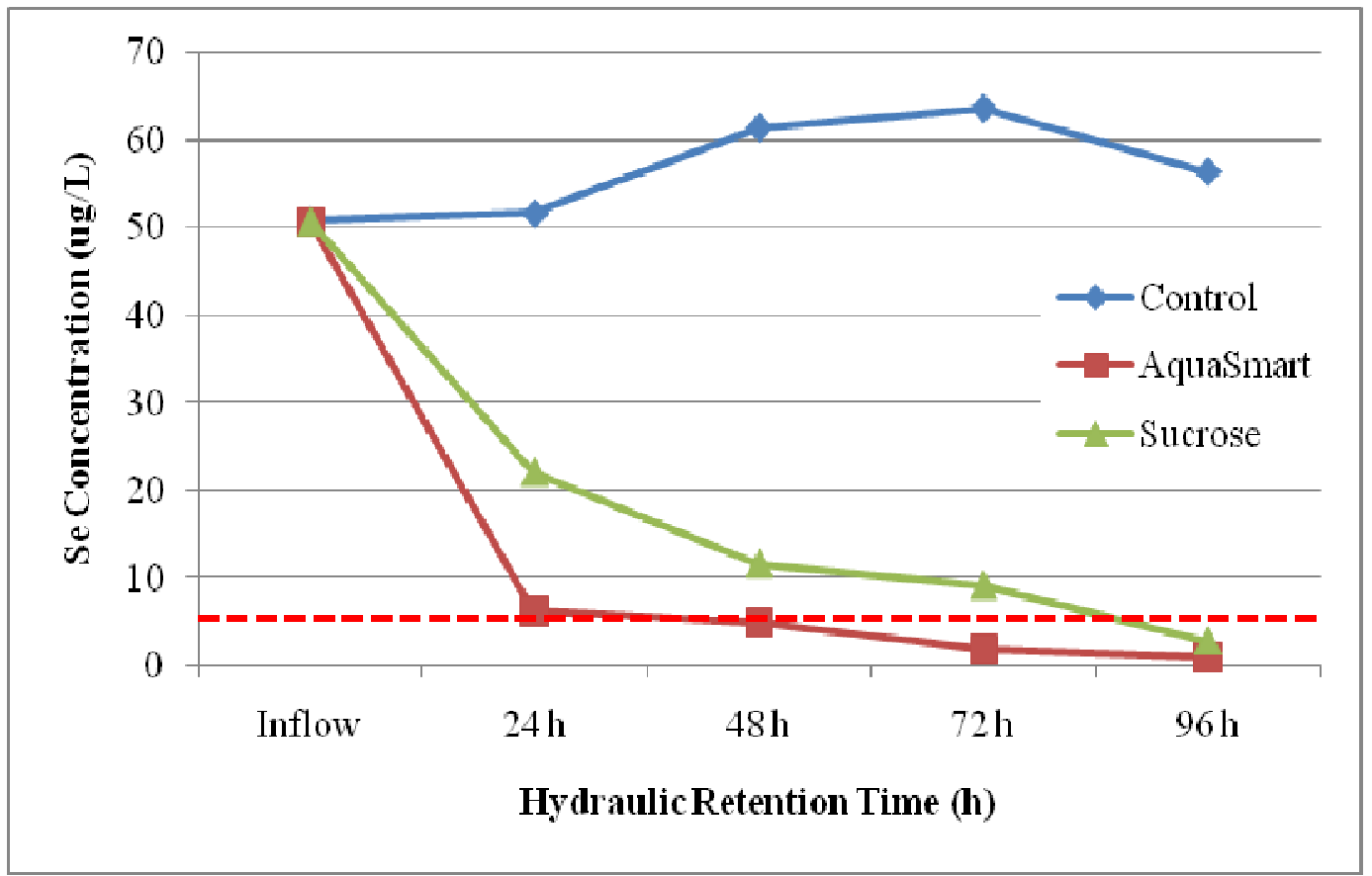

Figure 22. Se concentration during sampling period 6 with a goal of $5 \mu \mathrm{g} \mathrm{Se} / \mathrm{L}$ (red dashed line) 


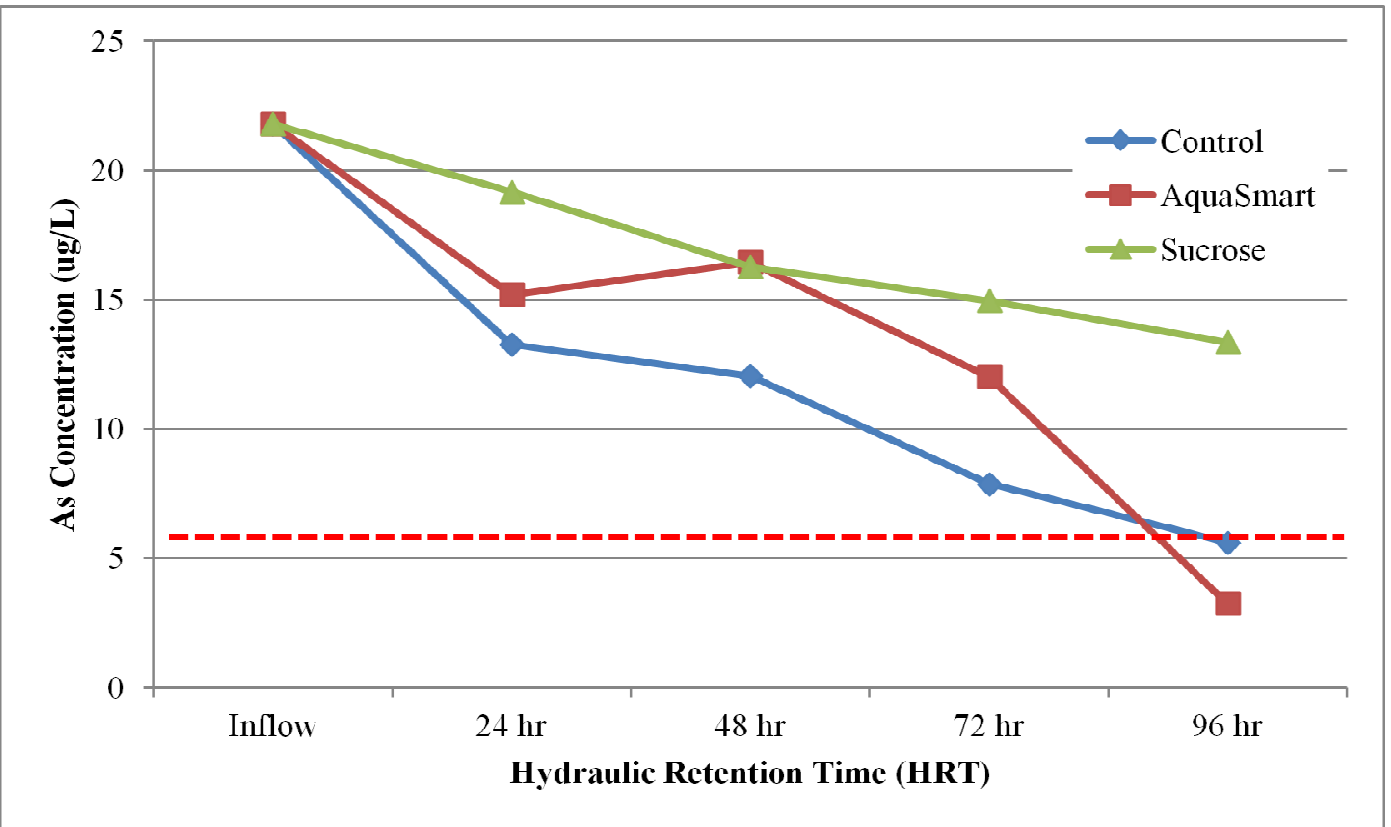

Figure 23. As concentration during sampling period 1 with a goal of $5 \mu \mathrm{g} \mathrm{As} / \mathrm{L}$ (red dashed line)

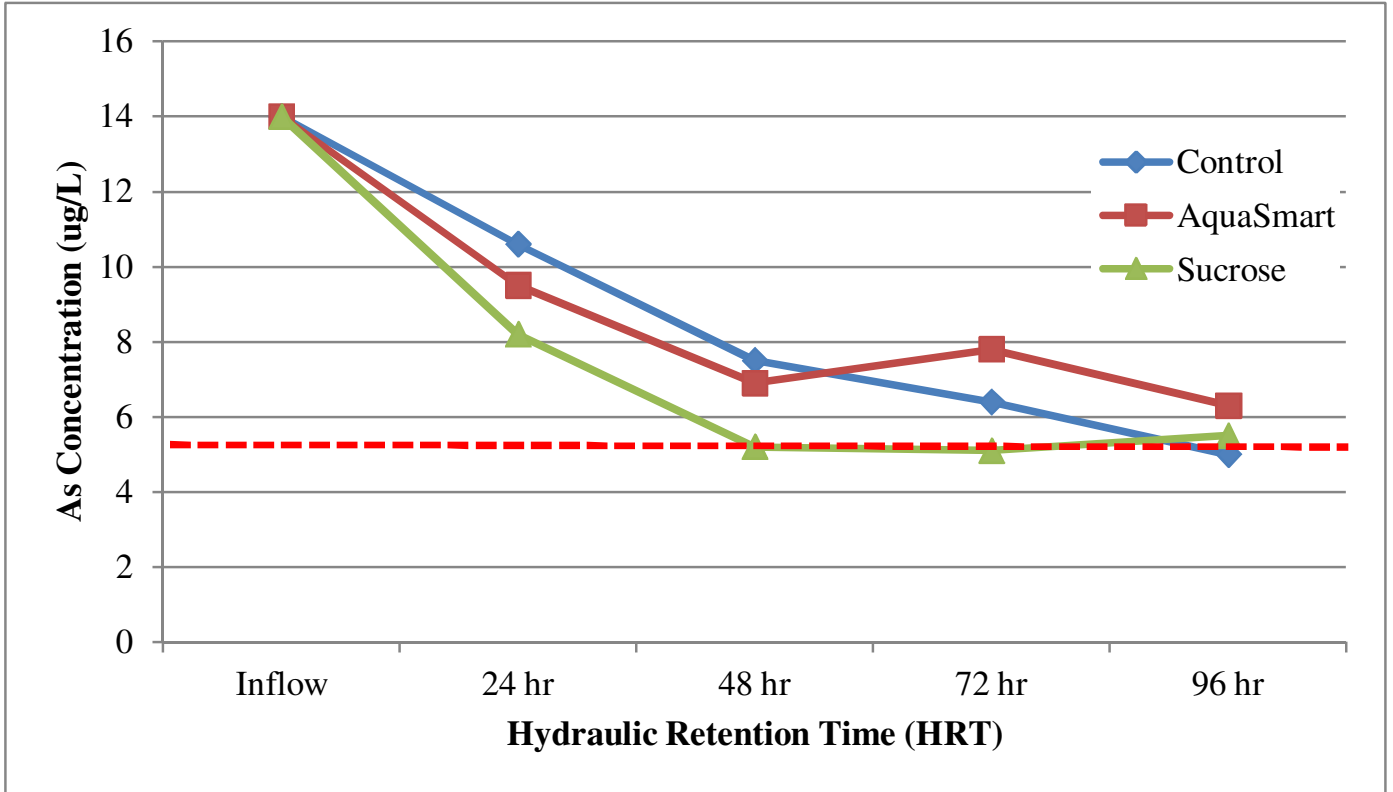

Figure 24. As concentration during sampling period 2 with a goal of $5 \mu \mathrm{g} \mathrm{As} / \mathrm{L}$ (red dashed line) 


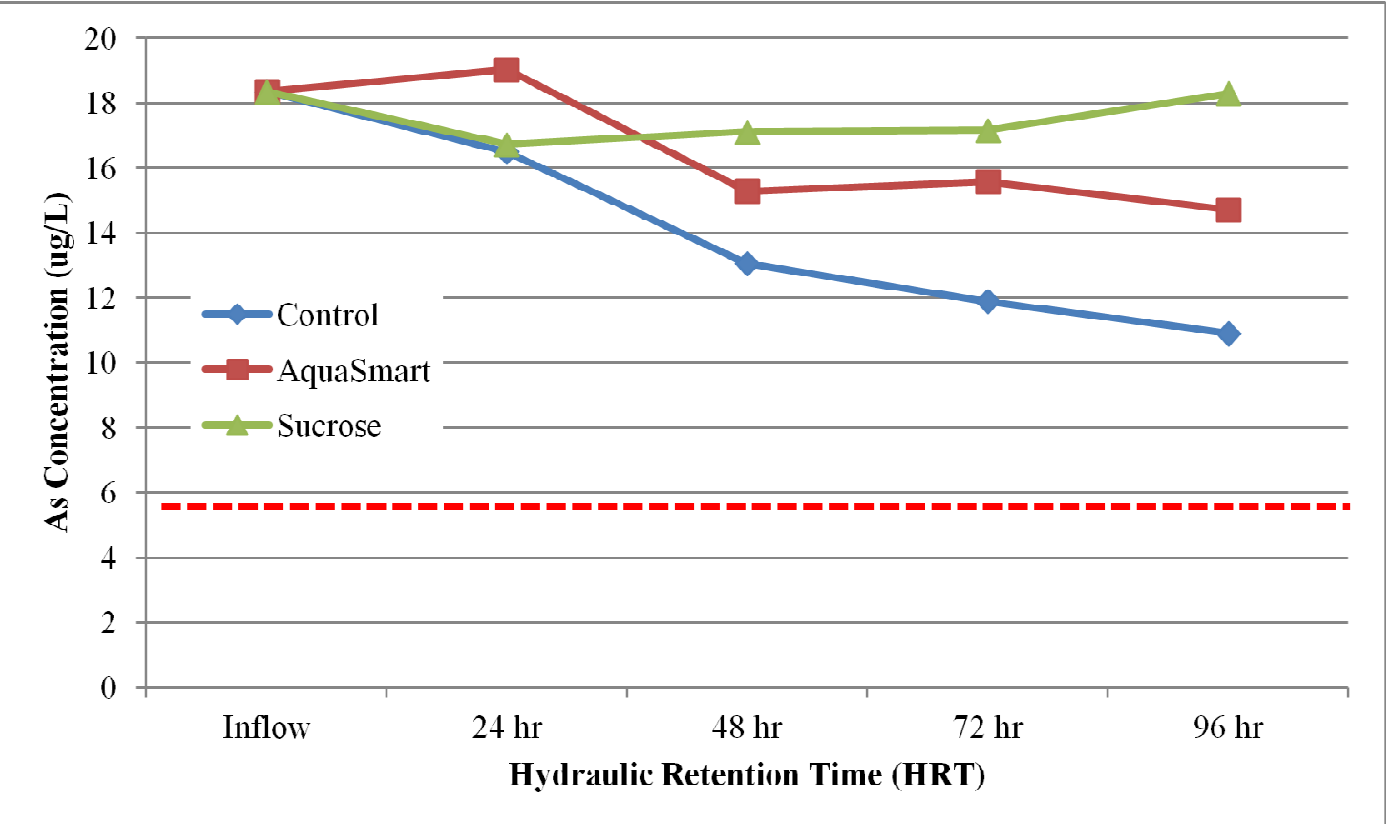

Figure 25. As concentration during sampling period 3 with a goal of $5 \mu \mathrm{g} \mathrm{As} / \mathrm{L}$ (red dashed line)

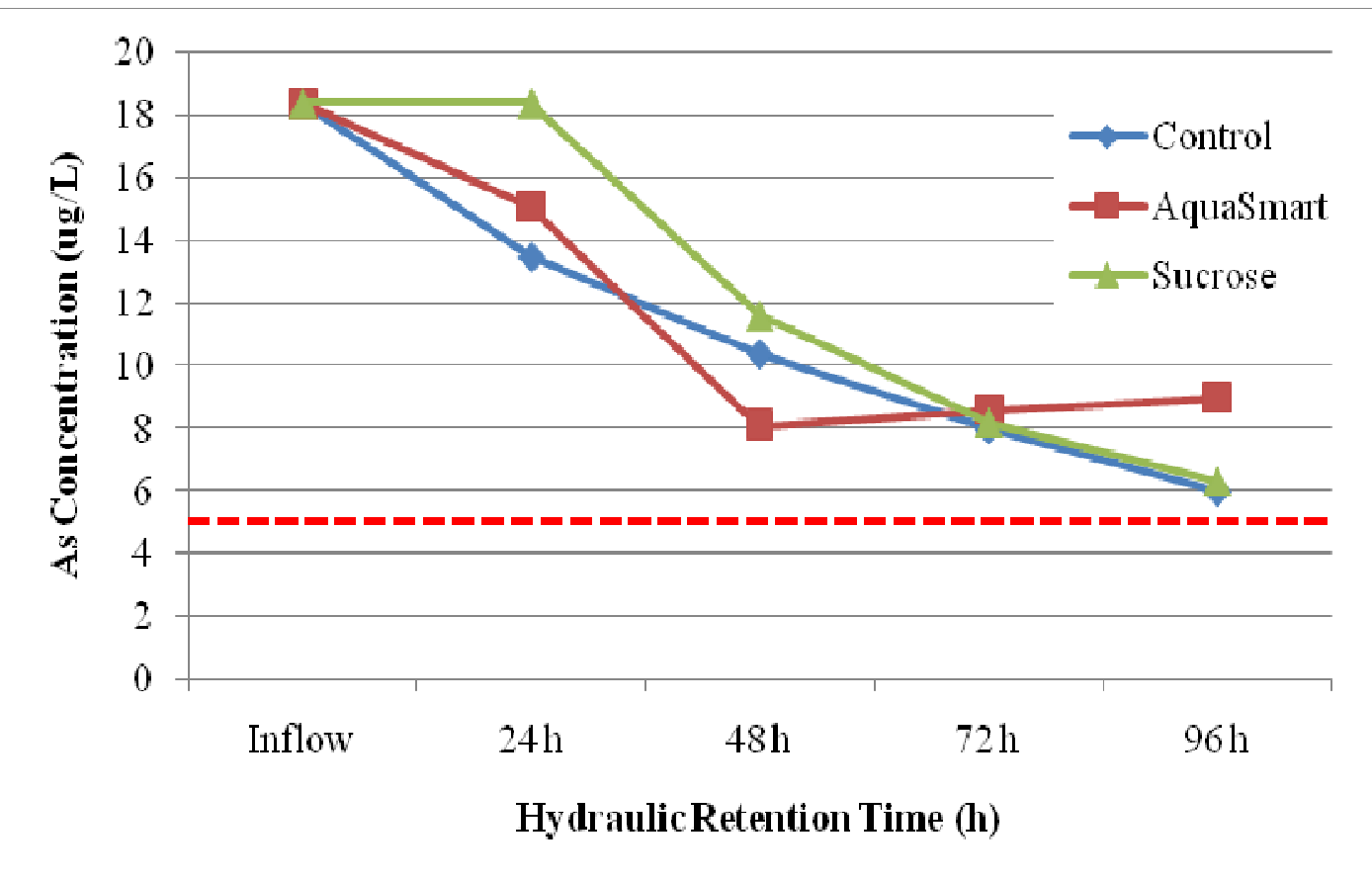

Figure 26. As concentration during sampling period 4 with a goal of $5 \mu \mathrm{g} \mathrm{As} / \mathrm{L}$ (red dashed line) 


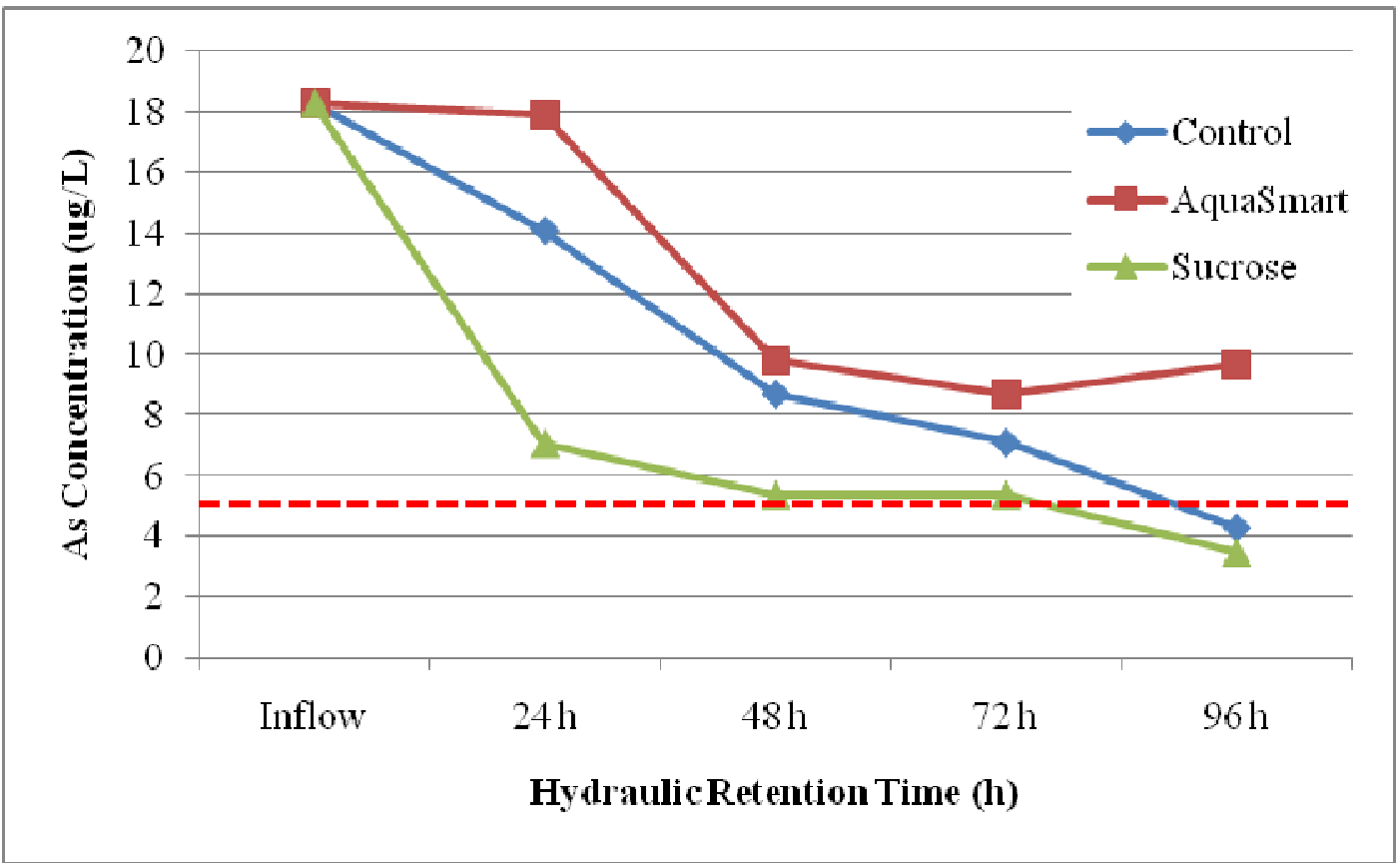

Figure 27. As concentration during sampling period 5 with a goal of $5 \mu \mathrm{g}$ As/L (red dashed line)

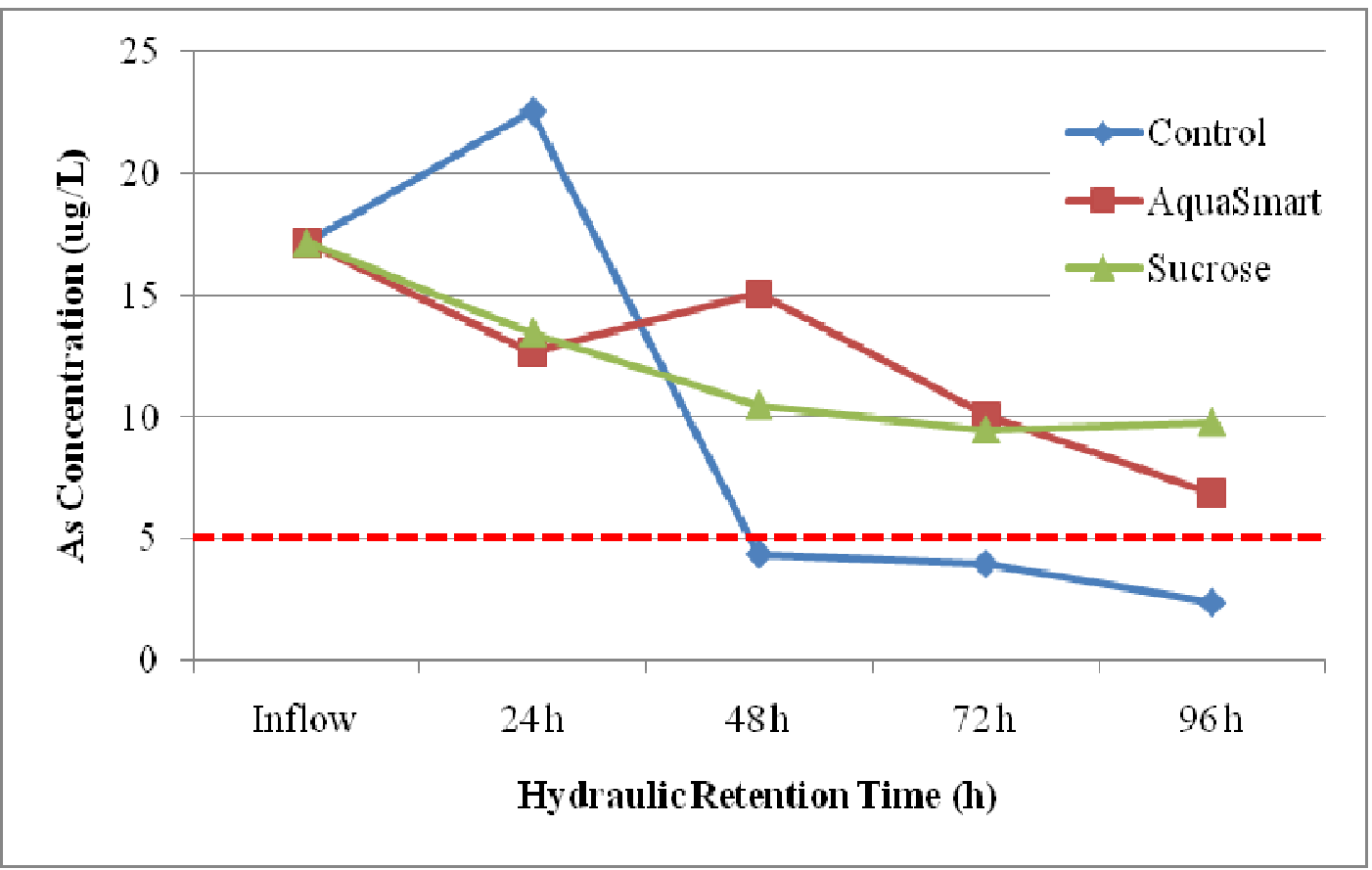

Figure 28. As concentration during sampling period 6 with a goal of $5 \mu \mathrm{g}$ As/L (red dashed line) 


\section{Pilot-Scale CWTS for PW Containing Oil \& Grease and Divalent Metals}

To determine treatment performance of the pilot-scale CWTSs, water samples were collected from five sampling locations in each wetland series. Sampling locations included inflow to the CWTS and after each cell A, B, C, and D (final effluent). Samples were collected following a sampling interval reflecting the HRT (24h). Samples collected were analyzed for total concentrations of metals ( $\mathrm{Fe}, \mathrm{Mn}, \mathrm{Ni}$, and $\mathrm{Zn}$ ), oil and grease $(\mathrm{O} \& \mathrm{G})$ concentration, and other water quality (explanatory) parameters. Total metal concentrations were determined by inductively coupled plasma-atomic emissions spectrometry (ICP-AES, EPA Method 200.7). Quality assurance and control measures included standard additions. Oil and grease concentration was determined by extraction with n-hexane using a StepSaver apparatus manufactured by Environmental Express (modification of EPA Method 1664A). Explanatory parameters were measured using Standard Methods (APHA, 1998) and included measurements of temperature, $\mathrm{pH}$, conductivity, alkalinity, hardness, and dissolved oxygen. Oxidationreduction (redox) potential of wetland hydrosoil was measured using milli-volt meters, an Accumet ${ }^{\circledR}$ calomel reference electrode, and in situ platinum-tipped electrodes (Faulkner et al., 1989).

Explanatory parameters including temperature, $\mathrm{pH}$, conductivity, alkalinity, hardness, and $\mathrm{DO}$ for sampling period 1-6 (Tables 45-50) were measured in the CWTSs because of their influence on wetland system pathways and constituent behavior. The same amount of $\mathrm{NaHCO}_{3}$, $\mathrm{MgSO}_{4} \bullet 7 \mathrm{H}_{2} \mathrm{O}, \mathrm{KNO}_{3}$, and $\mathrm{CaCO}_{3}$ was added to the simulated $\mathrm{PW}$ over the study period. These chemical sources were added to simulate ionic composition, hardness, and $\mathrm{pH}$ of PW. The pilotscale CWTSs were located in a greenhouse during the experiments, the water temperature ranged from approximately $19-27^{\circ} \mathrm{C}$ over the course of the study.

Redox potential was measured in each cell. Redox conditions affect chemical and microbial processes, and can greatly influence the biological availability of major and trace nutrients (Kadlec and Wallace, 2009). Measured redox potential in cell A of the SSF1 system indicates oxidizing conditions $(246 \mathrm{mV})$. Cells B and C of the SSF1 system had variable redox values (51 and $-14 \mathrm{mV})$, and cell $\mathrm{D}$ indicates oxidizing conditions $(98 \mathrm{mV})$. For the FWS series redox potential in cell A indicates oxidizing conditions $(82 \mathrm{mV})$, cells $\mathrm{B}$ and $\mathrm{C}$ range from slightly reducing $(-55 \mathrm{mV})$ to slightly oxidizing $(25 \mathrm{mV})$, and cell $\mathrm{D}$ is oxidizing $(80 \mathrm{mV})$. Dissolved oxygen concentrations for sampling period 1 decreased from $7.99 \mathrm{mg} / \mathrm{L}$ measured in the influent to $(5.89,6.5, \& 7.38 \mathrm{mg} / \mathrm{L})$ measured after the first cell of each respective system (Table 45$)$.

For sampling periods $4-6$ water temperature ranged from approximately $26-28^{\circ} \mathrm{C}$ over the course of the study. For sampling period 4, redox potential measured in cell A of the SSF1 system indicated oxidizing conditions $(210 \mathrm{mV})$. Redox potential ranged from -28 to $-101 \mathrm{mV}$ in cells $\mathrm{B}$ and C of the SSF1 system, and redox was $189 \mathrm{mV}$ in cell D. For the FWS series oxidizing conditions $(99 \mathrm{mV})$ were measured in cell $\mathrm{A}$, reducing $(-101 \mathrm{mV})$ to slightly oxidizing $(49 \mathrm{mV})$ conditions in cells B and C, and oxidizing conditions $(102 \mathrm{mV})$ in cell $\mathrm{D}$. For each respective series dissolved oxygen concentrations for sampling period 4 decreased from $7.72 \mathrm{mg} / \mathrm{L}$ in the influent to $6.23,7.1$, and $6.89 \mathrm{mg} / \mathrm{L}$ in the effluent of cell A (Table 48). Explanatory parameters for sampling periods 5 and 6 exhibited trends similar to those observed in sampling period 4 . 
The concentration of oil and grease $(O \& G)$ decreased to below guideline concentrations for irrigation and livestock watering after treatment in the pilot-scale CWTSs (HRT $=4$ days) for the subsurface flow series and free-water surface series operating at $50 \mathrm{mg} / \mathrm{L}$ (Table 47) and 100 $\mathrm{mg} / \mathrm{L}$ O\&G target influent (Tables 45,46 and 48-50). At $50 \mathrm{mg} / \mathrm{L}$ influent O\&G concentration, a 24-hr HRT was sufficient to meet guidelines for irrigation and livestock watering.

The concentrations of iron, manganese, nickel, and zinc in simulated PW decreased to below irrigation and livestock watering guidelines after treatment in the pilot-scale CWTSs (HRT 4days). Removal of iron, manganese, nickel, and zinc was greater in subsurface systems compared to that of the free water system. 
Table 45. Fe, Mn, Ni, Zn, O\&G concentrations and explanatory parameters for Sampling Period 1

\begin{tabular}{|c|c|c|c|c|c|c|c|c|c|c|c|c|}
\hline \multirow[b]{2}{*}{ Sample } & \multicolumn{5}{|c|}{ Performance Parameters } & \multicolumn{7}{|c|}{ Explanatory Parameters } \\
\hline & $\begin{array}{c}{[\mathrm{Fe}]} \\
(\mu \mathrm{g} / \mathrm{L})\end{array}$ & $\begin{array}{c}{[\mathrm{Mn}]} \\
(\mu \mathrm{g} / \mathrm{L})\end{array}$ & $\begin{array}{c}{[\mathrm{Ni}]} \\
(\mu \mathrm{g} / \mathrm{L}) \\
\end{array}$ & $\begin{array}{c}{[\mathrm{Zn}]} \\
(\mathrm{mg} / \mathrm{L}) \\
\end{array}$ & $\begin{array}{c}\text { [Oil \& Grease] } \\
(\mathrm{mg} / \mathrm{L})\end{array}$ & $\begin{array}{c}\mathrm{DO} \\
(\mathrm{mg} / \mathrm{L}) \\
\end{array}$ & $\begin{array}{l}\text { Cond. } \\
(\mu \mathrm{S} / \mathrm{cm})\end{array}$ & $\begin{array}{c}\mathrm{pH} \\
\text { (S.U.) }\end{array}$ & $\begin{array}{c}\text { Redox } \\
(\mathrm{mV})\end{array}$ & $\begin{array}{c}\text { Temp } \\
\left({ }^{\circ} \mathrm{C}\right)\end{array}$ & $\begin{array}{c}\text { Alkalinity } \\
(\mathrm{mg} / \mathrm{L} \\
\left.\mathrm{CaCO}_{3}\right) \\
\end{array}$ & $\begin{array}{c}\text { Hardness } \\
\left(\mathrm{mg} / \mathrm{L} \mathrm{CaCO}_{3}\right)\end{array}$ \\
\hline Inflow & 547.0 & 480.7 & 346.7 & 6.48 & 97.6 & 7.99 & 199 & 7.16 & nd & 24.2 & 38 & 45 \\
\hline FWS A & 182.5 & 151.7 & 308.4 & 4.35 & 11.5 & 5.89 & 201 & 6.62 & 82 & 26.9 & 40 & 36 \\
\hline FWS B & 183.2 & 129.7 & 267.0 & 2.23 & 7.6 & 4.58 & 204 & 6.79 & -55 & 26.5 & 45 & 38 \\
\hline FWS C & 84.4 & 34.5 & 273.6 & 1.65 & 4.8 & 4.61 & 207 & 6.81 & 25 & 27.1 & 42 & 37 \\
\hline FWS D & 58.8 & 16.1 & 266.7 & 1.04 & 3.2 & 7.65 & 210 & 6.92 & 80 & 27.4 & 48 & 41 \\
\hline SSF1 A & 46.4 & 6.0 & 255.3 & 0.566 & 25.4 & 6.5 & 200 & 6.88 & 246 & 26.6 & 35 & 21 \\
\hline SSF1 B & 25.0 & 2.9 & 221.9 & 0.153 & 13.8 & 7.1 & 204 & 6.79 & -51 & 26.8 & 39 & 25 \\
\hline SSF1 C & 15.2 & 2.0 & 152.3 & 0.051 & 8.6 & 7.13 & 209 & 6.84 & -14 & 26.8 & 31 & 28 \\
\hline SSF1 D & 16.7 & 2.9 & 116.7 & 0.023 & 4.2 & 7.38 & 215 & 6.94 & 98 & 26.3 & 47 & 37 \\
\hline SSF2 A & 40.6 & 6.8 & 249.6 & 0.454 & 30.6 & 6.21 & 213 & 6.83 & 212 & 24.1 & 39 & 28 \\
\hline SSF2 B & 18.7 & 3.2 & 193.5 & 0.144 & 18.9 & 6.15 & 227 & 6.89 & -65 & 25.3 & 45 & 31 \\
\hline SSF2 C & 16.5 & 2.9 & 154.2 & 0.060 & 9.2 & 7.1 & 222 & 6.95 & 15 & 25.7 & 35 & 35 \\
\hline SSF2 D & 14.5 & 2.4 & 117.9 & 0.029 & 3.8 & 6.89 & 229 & 6.78 & 73 & 24.9 & 41 & 41 \\
\hline
\end{tabular}

nd - not determined 
Table 46. Fe, Mn, Ni, Zn, O\&G concentrations and explanatory parameters for Sampling Period 2

\begin{tabular}{|c|c|c|c|c|c|c|c|c|c|c|c|c|}
\hline \multirow[b]{2}{*}{ Sample } & \multicolumn{5}{|c|}{ Performance Parameters } & \multicolumn{7}{|c|}{ Explanatory Parameters } \\
\hline & $\begin{array}{c}{[\mathrm{Fe}]} \\
(\mu \mathrm{g} / \mathrm{L}) \\
\end{array}$ & $\begin{array}{c}{[\mathrm{Mn}]} \\
(\mu \mathrm{g} / \mathrm{L})\end{array}$ & $\begin{array}{c}{[\mathrm{Ni}]} \\
(\mu \mathrm{g} / \mathrm{L}) \\
\end{array}$ & $\begin{array}{c}{[\mathrm{Zn}]} \\
(\mathrm{mg} / \mathrm{L}) \\
\end{array}$ & $\begin{array}{c}\text { [Oil \& Grease] } \\
(\mathrm{mg} / \mathrm{L})\end{array}$ & $\begin{array}{c}\mathrm{DO} \\
(\mathrm{mg} / \mathrm{L})\end{array}$ & $\begin{array}{l}\text { Cond. } \\
(\mu \mathrm{S} / \mathrm{cm})\end{array}$ & $\begin{array}{c}\mathrm{pH} \\
(\mathrm{S} . \mathrm{U} .) \\
\end{array}$ & $\begin{array}{c}\text { Redox } \\
(\mathrm{mV})\end{array}$ & $\begin{array}{l}\text { Temp } \\
\left({ }^{\circ} \mathrm{C}\right)\end{array}$ & $\begin{array}{c}\text { Alkalinity } \\
(\mathrm{mg} / \mathrm{L} \\
\left.\mathrm{CaCO}_{3}\right) \\
\end{array}$ & $\begin{array}{c}\text { Hardness } \\
(\mathrm{mg} / \mathrm{L} \\
\left.\mathrm{CaCO}_{3}\right)\end{array}$ \\
\hline Inflow & 296.5 & 409.6 & 312.8 & 4.74 & 94.2 & 7.91 & 214 & 6.87 & nd & 24.4 & 45 & 42 \\
\hline FWS A & 157.3 & 183.6 & 243.4 & 3.54 & 13.4 & 5.55 & 216 & 6.45 & 32 & 25.9 & 41 & 29 \\
\hline FWS B & 81.0 & 113.0 & 246.0 & 3.63 & 10.1 & 5.82 & 211 & 6.52 & -79 & 26.4 & 43 & 35 \\
\hline FWS C & 70.8 & 53.3 & 219.5 & 3.16 & 6.9 & 6.13 & 210 & 6.66 & -101 & 26.6 & 47 & 33 \\
\hline FWS D & 71.7 & 32.4 & 180.7 & 2.56 & 2.3 & 6.01 & 217 & 6.59 & 15 & 25.9 & 44 & 38 \\
\hline SSF1 A & 18.5 & 3.4 & 221.2 & 2.64 & 37.2 & 6.31 & 210 & 7.01 & 121 & 24.1 & 39 & 28 \\
\hline SSF1 B & 16.8 & 2.7 & 190.6 & 2.05 & 19.1 & 6.14 & 209 & 6.95 & -112 & 25.9 & 48 & 33 \\
\hline SSF1 C & 13.0 & 3.3 & 135.6 & 1.15 & 12.9 & 6.41 & 216 & 6.83 & -130 & 26.1 & 45 & 38 \\
\hline SSF1 D & 10.9 & 3.0 & 109.0 & 0.791 & 5.9 & 6.87 & 213 & 6.91 & -54 & 25.1 & 51 & 41 \\
\hline SSF2 A & 19.5 & 3.6 & 207.1 & 2.60 & 12.9 & 6.5 & 217 & 6.42 & 98 & 23.9 & 26 & 31 \\
\hline SSF2 B & 14.6 & 2.9 & 175.7 & 1.97 & 11.3 & 6.34 & 223 & 6.66 & -82 & 24.1 & 28 & 29 \\
\hline SSF2 C & 10.8 & 2.7 & 147.0 & 1.48 & 8.2 & 6.79 & 221 & 6.49 & -23 & 23.5 & 37 & 37 \\
\hline SSF2 D & 13.3 & 2.7 & 112.1 & 0.955 & 4.7 & 7.07 & 226 & 6.78 & 11 & 23.1 & 35 & 41 \\
\hline
\end{tabular}

nd - not determined 
Table 47. O\&G concentrations and explanatory parameters for Sampling Period 3

\begin{tabular}{|c|c|c|c|c|c|c|c|c|}
\hline \multirow[b]{2}{*}{ Sample } & \multirow{2}{*}{$\begin{array}{l}\text { Performance Parameters } \\
\text { [Oil \& Grease] (mg/L) }\end{array}$} & \multicolumn{7}{|c|}{ Explanatory Parameters } \\
\hline & & $\begin{array}{c}\mathrm{DO} \\
(\mathrm{mg} / \mathrm{L})\end{array}$ & $\begin{array}{l}\text { Cond. } \\
(\mu \mathrm{S} / \mathrm{cm})\end{array}$ & $\begin{array}{c}\mathrm{pH} \\
(\mathrm{S} . \mathrm{U} .)\end{array}$ & $\begin{array}{c}\text { Redox } \\
(\mathrm{mV})\end{array}$ & $\begin{array}{c}\text { Temp } \\
\left({ }^{\circ} \mathrm{C}\right)\end{array}$ & $\begin{array}{l}\text { Alkalinity } \\
(\mathrm{mg} / \mathrm{L} \\
\left.\mathrm{CaCO}_{3}\right)\end{array}$ & $\begin{array}{c}\text { Hardness } \\
(\mathrm{mg} / \mathrm{L} \\
\left.\mathrm{CaCO}_{3}\right)\end{array}$ \\
\hline Inflow & 52.3 & 8.17 & 173 & 6.1 & nd & 20.1 & 29 & 44 \\
\hline FWS A & 9.9 & 7.56 & 176 & 6.19 & -120 & 20.9 & 31 & 42 \\
\hline FWS B & 4.3 & 7.39 & 174 & 6.04 & -23 & 20.4 & 27 & 30 \\
\hline FWS C & 3.6 & 7.73 & 170 & 6.21 & 31 & 20.3 & 33 & 37 \\
\hline FWS D & 2.2 & 8.02 & 178 & 6.13 & 40 & 19.9 & 39 & 48 \\
\hline SSF1 A & 12.1 & 6.77 & 176 & 6.03 & -72 & 19.7 & 22 & 41 \\
\hline SSF1 B & 10.3 & 6.97 & 172 & 6.15 & -99 & 20.4 & 25 & 29 \\
\hline SSF1 C & 7.6 & 6.63 & 180 & 6.17 & -31 & 20.7 & 27 & 33 \\
\hline SSF1 D & 2.1 & 7.23 & 178 & 6.21 & 41 & 20 & 31 & 30 \\
\hline SSF2 A & 12.6 & 6.03 & 198 & 6.09 & -69 & 19.8 & 45 & 32 \\
\hline SSF2 B & 9.8 & 5.99 & 201 & 6.11 & -201 & 20.1 & 39 & 37 \\
\hline $\mathrm{SSF} 2 \mathrm{C}$ & 4.5 & 6.19 & 195 & 6.23 & -132 & 21.2 & 53 & 34 \\
\hline SSF2 D & 2.9 & 6.31 & 208 & 6.2 & -29 & 20.6 & 47 & 35 \\
\hline
\end{tabular}

nd - not determined 
Table 48. Fe, Mn, Ni, Zn, O\&G concentrations and explanatory parameters for Sampling Period 4

\begin{tabular}{|c|c|c|c|c|c|c|c|c|c|c|c|c|}
\hline \multirow[b]{2}{*}{ Sample } & \multicolumn{5}{|c|}{ Performance Parameters } & \multicolumn{7}{|c|}{ Explanatory Parameters } \\
\hline & $\begin{array}{c}{[\mathrm{Fe}]} \\
(\mu \mathrm{g} / \mathrm{L})\end{array}$ & $\begin{array}{c}{[\mathrm{Mn}]} \\
(\mu \mathrm{g} / \mathrm{L})\end{array}$ & $\begin{array}{c}{[\mathrm{Ni}]} \\
(\mu \mathrm{g} / \mathrm{L})\end{array}$ & $\begin{array}{c}{[\mathrm{Zn}]} \\
(\mathrm{mg} / \mathrm{L})\end{array}$ & $\begin{array}{c}\text { [Oil \& Grease] } \\
(\mathrm{mg} / \mathrm{L})\end{array}$ & $\begin{array}{c}\mathrm{DO} \\
(\mathrm{mg} / \mathrm{L})\end{array}$ & $\begin{array}{l}\text { Cond. } \\
(\mu \mathrm{S} / \mathrm{cm})\end{array}$ & $\begin{array}{c}\mathrm{pH} \\
\text { (S.U.) }\end{array}$ & $\begin{array}{c}\text { Redox } \\
(\mathrm{mV})\end{array}$ & $\begin{array}{l}\text { Temp } \\
\left({ }^{\circ} \mathrm{C}\right)\end{array}$ & $\begin{array}{c}\text { Alkalinity } \\
(\mathrm{mg} / \mathrm{L} \\
\left.\mathrm{CaCO}_{3}\right)\end{array}$ & $\begin{array}{c}\text { Hardness } \\
\left(\mathrm{mg} / \mathrm{L} \mathrm{CaCO}_{3}\right)\end{array}$ \\
\hline Inflow & 286.7 & 510.2 & 322.2 & 5.23 & 101.2 & 7.72 & 221 & 7.21 & nd & 27.1 & 42 & 40 \\
\hline FWS A & 179.6 & 193.2 & 299.2 & 4.89 & 29.1 & 6.23 & 215 & 6.88 & 99 & 26.5 & 49 & 41 \\
\hline FWS B & 163.2 & 152.1 & 278.2 & 3.67 & 17.1 & 4.98 & 214 & 6.67 & -101 & 26.9 & 42 & 48 \\
\hline FWS C & 97.8 & 59.7 & 261.9 & 2.01 & 8.9 & 4.99 & 203 & 6.72 & 49 & 27.6 & 47 & 39 \\
\hline FWS D & 64.4 & 31.1 & 255.2 & 1.01 & 7.1 & 7.78 & 212 & 6.85 & 102 & 27.9 & 46 & 43 \\
\hline SSF1 A & 52.2 & 9.2 & 270.1 & 0.633 & 28.2 & 7.1 & 208 & 6.91 & 210 & 27.1 & 39 & 32 \\
\hline SSF1 B & 29.7 & 5.6 & 211.1 & 0.545 & 19.1 & 7.21 & 209 & 6.85 & -28 & 27.2 & 41 & 29 \\
\hline SSF1 C & 18.2 & 6.1 & 189.1 & 0.314 & 11.1 & 7.78 & 213 & 6.92 & -101 & 26.9 & 48 & 27 \\
\hline SSF1 D & 15.2 & 4.3 & 139.2 & 0.289 & 5.6 & 7.67 & 217 & 6.99 & 189 & 27.1 & 39 & 34 \\
\hline SSF2 A & 38.7 & 5.1 & 263.3 & 0.685 & 33.2 & 6.89 & 223 & 6.93 & 267 & 26.9 & 34 & 26 \\
\hline SSF2 B & 21.4 & 3.9 & 201.2 & 0.233 & 15.2 & 6.76 & 229 & 7.11 & -103 & 26.1 & 31 & 36 \\
\hline $\mathrm{SSF} 2 \mathrm{C}$ & 19.7 & 2.8 & 170.4 & 0.123 & 10.9 & 7.98 & 231 & 6.92 & 65 & 26.6 & 39 & 39 \\
\hline SSF2 D & 12.1 & 3.1 & 138.2 & 0.111 & 4.2 & 6.57 & 227 & 6.89 & 99 & 25.9 & 44 & 31 \\
\hline
\end{tabular}

nd - not determined 
Table 49. Fe, Mn, Ni, Zn, O\&G concentrations and explanatory parameters for Sampling Period 5

\begin{tabular}{|c|c|c|c|c|c|c|c|c|c|c|c|c|}
\hline \multirow[b]{2}{*}{ Sample } & \multicolumn{5}{|c|}{ Performance Parameters } & \multicolumn{7}{|c|}{ Explanatory Parameters } \\
\hline & $\begin{array}{c}{[\mathrm{Fe}]} \\
(\mu \mathrm{g} / \mathrm{L})\end{array}$ & $\begin{array}{c}{[\mathrm{Mn}]} \\
(\mu \mathrm{g} / \mathrm{L})\end{array}$ & $\begin{array}{c}{[\mathrm{Ni}]} \\
(\mu \mathrm{g} / \mathrm{L})\end{array}$ & $\begin{array}{c}{[\mathrm{Zn}]} \\
(\mathrm{mg} / \mathrm{L})\end{array}$ & $\begin{array}{c}\text { [Oil \& Grease] } \\
(\mathrm{mg} / \mathrm{L})\end{array}$ & $\begin{array}{c}\mathrm{DO} \\
(\mathrm{mg} / \mathrm{L})\end{array}$ & $\begin{array}{l}\text { Cond. } \\
(\mu \mathrm{S} / \mathrm{cm})\end{array}$ & $\begin{array}{c}\mathrm{pH} \\
(\mathrm{S} . \mathrm{U} .)\end{array}$ & $\begin{array}{c}\text { Redox } \\
(\mathrm{mV})\end{array}$ & $\begin{array}{l}\text { Temp } \\
\left({ }^{\circ} \mathrm{C}\right)\end{array}$ & $\begin{array}{c}\text { Alkalinity } \\
(\mathrm{mg} / \mathrm{L} \\
\left.\mathrm{CaCO}_{3}\right)\end{array}$ & $\begin{array}{c}\text { Hardness } \\
(\mathrm{mg} / \mathrm{L} \\
\left.\mathrm{CaCO}_{3}\right)\end{array}$ \\
\hline Inflow & 320.4 & 463.2 & 367.9 & 6.23 & 95.9 & 7.44 & 209 & 6.81 & nd & 26.4 & 41 & 37 \\
\hline FWS A & 190.5 & 224.1 & 262.2 & 4.52 & 15.2 & 6.01 & 201 & 6.55 & -56 & 26.3 & 48 & 35 \\
\hline FWS B & 178.9 & 109.3 & 239.3 & 3.09 & 9.5 & 6.11 & 205 & 6.51 & -160 & 26.9 & 41 & 39 \\
\hline FWS C & 104.3 & 81.4 & 199.5 & 2.58 & 5.4 & 6.19 & 209 & 6.76 & -23 & 27.1 & 39 & 32 \\
\hline FWS D & 73.2 & 50.2 & 191.3 & 1.97 & 4.2 & 5.99 & 213 & 6.81 & 67 & 27.3 & 43 & 37 \\
\hline SSF1 A & 98.2 & 9.7 & 245.6 & 1.21 & 31.1 & 6.22 & 207 & 6.99 & 48 & 27.2 & 37 & 29 \\
\hline SSF1 B & 31.5 & 8.9 & 209.8 & 0.735 & 22.8 & 6.29 & 211 & 6.89 & -100 & 26.9 & 41 & 36 \\
\hline SSF1 C & 17.7 & 9.3 & 142.2 & 0.747 & 18.1 & 6.19 & 213 & 7.01 & 45 & 26.6 & 44 & 35 \\
\hline SSF1 D & 12.2 & 4.7 & 129.2 & 0.587 & 9.9 & 6.73 & 219 & 7.03 & 10 & 27.5 & 48 & 39 \\
\hline SSF2 A & 29.9 & 7.8 & 227.2 & 1.5 & 21.1 & 5.87 & 212 & 6.58 & 101 & 26.2 & 29 & 33 \\
\hline SSF2 B & 23.2 & 7.9 & 174.9 & 0.897 & 19.4 & 6.15 & 217 & 6.71 & -120 & 26.5 & 31 & 26 \\
\hline $\mathrm{SSF} 2 \mathrm{C}$ & 11.1 & 3.9 & 153.2 & 0.777 & 15.3 & 6.66 & 225 & 6.88 & -89 & 26.5 & 28 & 31 \\
\hline SSF2 D & 10.4 & 2.1 & 135.1 & 0.619 & 7.2 & 6.92 & 221 & 6.52 & 49 & 26.8 & 31 & 42 \\
\hline
\end{tabular}

nd - not determined 
Table 50. O\&G concentrations and explanatory parameters for Sampling Period 6

\begin{tabular}{|c|c|c|c|c|c|c|c|c|}
\hline \multirow[b]{2}{*}{ Sample } & \multirow{2}{*}{$\begin{array}{l}\text { Performance Parameters } \\
\text { [Oil \& Grease] (mg/L) }\end{array}$} & \multicolumn{7}{|c|}{ Explanatory Parameters } \\
\hline & & $\begin{array}{c}\mathrm{DO} \\
(\mathrm{mg} / \mathrm{L})\end{array}$ & $\begin{array}{l}\text { Cond. } \\
(\mu \mathrm{S} / \mathrm{cm})\end{array}$ & $\begin{array}{c}\mathrm{pH} \\
\text { (S.U.) }\end{array}$ & $\begin{array}{c}\text { Redox } \\
(\mathrm{mV})\end{array}$ & $\begin{array}{l}\text { Temp } \\
\left({ }^{\circ} \mathrm{C}\right)\end{array}$ & $\begin{array}{l}\text { Alkalinity } \\
(\mathrm{mg} / \mathrm{L} \\
\left.\mathrm{CaCO}_{3}\right) \\
\end{array}$ & $\begin{array}{c}\text { Hardness } \\
(\mathrm{mg} / \mathrm{L} \\
\left.\mathrm{CaCO}_{3}\right)\end{array}$ \\
\hline Inflow & 99.7 & 8.09 & 189 & 6.5 & nd & 26.7 & 47 & 49 \\
\hline FWS A & 16.6 & 6.47 & 187 & 6.37 & -87 & 26.2 & 39 & 40 \\
\hline FWS B & 12.4 & 6.41 & 181 & 6.29 & 25 & 27.1 & 42 & 39 \\
\hline FWS C & 7.8 & 6.81 & 179 & 6.41 & 109 & 27.5 & 43 & 45 \\
\hline FWS D & 2.9 & 6.99 & 183 & 6.39 & 150 & 27.2 & 43 & 42 \\
\hline SSF1 A & 15.2 & 6.03 & 186 & 6.6 & 67 & 26.8 & 45 & 50 \\
\hline SSF1 B & 11.4 & 6.25 & 181 & 6.57 & -102 & 26.8 & 39 & 49 \\
\hline SSF1 C & 6.9 & 5.89 & 192 & 6.61 & -15 & 26.2 & 41 & 48 \\
\hline SSF1 D & 3.1 & 6.32 & 194 & 6.68 & 54 & 27.1 & 42 & 46 \\
\hline SSF2 A & 18.9 & 6.77 & 193 & 6.52 & 35 & 26.9 & 40 & 42 \\
\hline SSF2 B & 13.9 & 6.55 & 188 & 6.5 & -233 & 26.5 & 37 & 38 \\
\hline $\mathrm{SSF} 2 \mathrm{C}$ & 9.4 & 6.31 & 181 & 6.48 & -65 & 26.8 & 39 & 36 \\
\hline SSF2 D & 5.3 & 6.69 & 191 & 6.41 & 13 & 27.2 & 44 & 40 \\
\hline
\end{tabular}

nd - not determined 


\section{Assess Pilot-Scale Performance (Task 6)}

The objective of Task 6 is to assess performance of the pilot-scale CWTSs by (1) calculating removal rate coefficients, removal efficiencies, and removal extents and (2) comparing removal extents with selected reuse criteria.

Removal rate coefficients were calculated to determine the efficiency and effectiveness of each system's design in terms of removal processes and pathways and to allow for determination of critical design parameters including HRT. Removal rate coefficients $(k)$ were calculated assuming first order rate kinetics (Equation 4).

$$
\text { Removal rate coefficient }(k)=\frac{-\ln \left([C] /\left[C_{o}\right]\right)}{t}
$$

where, $\left[C_{\mathrm{o}}\right]$ is the initial inflow concentration $(\mathrm{mg} / \mathrm{L}),[C]$ is the system outflow concentration $(\mathrm{mg} / \mathrm{L})$, and $t$ is the time (hours) and corresponds to HRT of the system. Negative values for removal rates occur when outflow concentrations are greater than inflow concentrations. Removal efficiencies, defined as the percent decrease in outflow concentration relative to inflow concentration were calculated (Equation 5).

$$
\text { Removal efficiency }(\%)=\frac{\left[C_{o}\right]-[C]}{\left[C_{o}\right]} \times 100
$$

where, $\left[C_{\mathrm{o}}\right]$ is the initial inflow concentration $(\mathrm{mg} / \mathrm{L})$ and $[C]$ is the outflow concentration $(\mathrm{mg} / \mathrm{L})$. Removal extent is defined as the outflow concentration, and can be compared with selected reuse criteria to determine the success of the system performance.

\section{Pilot-Scale CWTS for Simulated Post-RO Produced Water}

Removal rates, efficiencies, and extents were calculated for ammonia for all sampling periods using Equations 4 and 5 (Table 51). Removal rate efficiencies were greater in the experimental system during all sampling periods indicating that ammonia removal by the targeted removal pathways including nitrification can be enhanced when the correct operating conditions are applied (Table 20). Removal extents suggest that the concentration ammonia in simulated post$\mathrm{RO}$ produced can be reduced to meet irrigation and livestock watering criteria. 
Table 51. Ammonia removal extents, efficiencies, and rate coefficients for all sampling periods.

\begin{tabular}{clccc}
\hline Sampling Period & Parameter & Conc. $(\mathrm{mg} / \mathrm{L})$ & Removal $(\%)$ & Rate Coef. $\left(\mathrm{d}^{-1}\right)$ \\
\hline \multirow{2}{*}{1} & Influent & 20.9 & -- & -- \\
& Control Extent & 17.6 & 15.8 & 0.043 \\
& Experimental Extent & 2.3 & 89.0 & 0.276 \\
\multirow{2}{*}{2} & Influent & 21.2 & -- & -- \\
& Control Extent & 16.7 & 21.2 & 0.060 \\
& Experimental Extent & 7.8 & 63.2 & 0.125 \\
\multirow{3}{*}{3} & Influent & 20.3 & -- & -- \\
& Control Extent & 14.3 & 29.6 & 0.088 \\
& Experimental Extent & 7.4 & 63.5 & 0.126 \\
\multirow{2}{*}{4} & Influent & 22.3 & -- & -- \\
& Control Extent & 12.6 & 43.5 & 0.071 \\
& Experimental Extent & $<0.1$ & $>99.6$ & 1.35 \\
& Influent & 23.2 & -- & -- \\
& Control Extent & 15 & 35.3 & 0.073 \\
& Experimental Extent & $<0.1$ & $>99.6$ & $1.36^{*}$ \\
& Influent & 25.7 & -- & -- \\
& Control Extent & 13.2 & 48.6 & 0.111 \\
& Experimental Extent & $<0.1$ & $>99.6$ & $1.39^{*}$ \\
\hline
\end{tabular}

*Rate coefficient calculated for 6 days of treatment; concentrations in cells 3 and 4 were identical.

Pilot-Scale CWTS for PW Containing Divalent Metals and Low Molecular Weight Organics

Removal rates, efficiencies, and extents of $\mathrm{Cd}^{+2}, \mathrm{Cu}^{+2}, \mathrm{Ni}^{+2}, \mathrm{Zn}^{+2}$, and LMWOs in the pilot-scale system were calculated to determine the efficiency and effectiveness of the system design in terms of removal processes and pathways for each cell using equations 4 and 5 (Table 52). Removal rate coefficients were calculated assuming first order kinetics; however, upon examination, not all data fit first order (i.e. Figures 9-10).

In sampling period 1, both series had calculated removal efficiencies less than $56 \%$ for COCs removed from simulated fresh PW. These removal efficiencies may be attributed to lack of maturity of the system. For sampling periods 2 through 5, majority of the removal efficiency values were $80 \%$ or greater. The increase from $56 \%$ to $80 \%$ removal efficiency for the series may be a result of maturity and acclimation to the simulated fresh PW in addition to increased microbial activity (i.e. dissimilatory sulfate reducers and heterotrophic aerobic microbes). Removal extent and beneficial use criteria and guidelines were compared to determine if the effluent from the CWTS met or exceeded the use criteria (Table 53). For 70\% of the sampling periods, copper concentrations met or were below all of the beneficial use guidelines and criteria. Cadmium concentrations met the beneficial uses for two sampling periods, while $80 \%$ of the nickel concentrations were below the surface water discharge criteria and $30 \%$ met the irrigation 
guidelines. Every zinc concentration met the livestock watering criteria, and irrigation guidelines and surface discharge criteria were met for all sampling periods except for sampling period 1. Concentrations of LMWOs were below all of the beneficial use guidelines and criteria for every sampling period.

\section{Pilot-Scale CWTS for PW Containing Metalloids and Low Molecular Weight Organics}

Removal rate coefficients, removal efficiencies, and removal extents of selenium and arsenic in the pilot-scale system were calculated to determine the efficiency and effectiveness of the system design in terms of removal processes and pathways for each cell using equations 4 and 5 (Table 54).

The goal of $5 \mu \mathrm{g} \mathrm{Se} / \mathrm{L}$ in the outflow water was achieved and maintained in the pilot-scale CWTS with the AquaSmart ${ }^{\mathrm{TM}}$ amendment. The sucrose amendment also achieved the goal of 5 $\mu \mathrm{g} \mathrm{Se} / \mathrm{L}$, but with less consistency than the AquaSmart ${ }^{\mathrm{TM}}$ amendment. The untreated control series did not meet the $5 \mu \mathrm{g} \mathrm{Se} / \mathrm{L}$ goal during any sampling periods. The goal of $5 \mu \mathrm{g} \mathrm{As} / \mathrm{L}$ in the outflow water was achieved by both the untreated control series and the AquaSmart ${ }^{\mathrm{TM}}$ series.

\section{Pilot-Scale CWTS for PW Containing Oil \& Grease and Divalent Metals}

The concentrations of iron, manganese, nickel, and zinc in simulated OFPW decreased to below irrigation and livestock watering guidelines by treatment in the pilot-scale CWTSs (HRT 4-days) designed for removal of oil and grease $(\mathrm{O} \& \mathrm{G})$. Removal efficiencies $(\%)$ and removal rate coefficients $(k)$ for iron, manganese, nickel, and zinc were greater in the subsurface flow series than in the free-water surface series (Table 55). Removal efficiencies and removal rate coefficients $(k)$ were greater for iron, manganese, and zinc than for nickel (Table 55).

The concentration of O\&G in simulated OFPW decreased to below irrigation and livestock watering guidelines by treatment in the pilot-scale CWTSs (HRT 4-days) in the subsurface flow series and free-water surface series operating at 50 and 100mg/L O\&G target influent (Table 56). There was no discernable difference in O\&G removal, measured through removal rate coefficients $(k)$ and removal efficiencies $(\%)$, between the subsurface flow and free-water surface series. Removal was greatest in the first cell of each series, with oil and grease concentrations reduced by fifty percent (from influent concentrations) in effluent samples collected from the first cell of each system. Effluent samples collected from the final cell of each series increased through the duration of the experiment; however, these respective concentrations remained below irrigation and livestock watering guidelines. 
Table 52. Removal Efficiency Calculations for $\mathrm{Cd}^{+2}, \mathrm{Cu}^{+2}, \mathrm{Ni}^{+2}, \mathrm{Zn}^{+2}$, and LMWOs in the Pilot-scale CWTS

\begin{tabular}{|c|c|c|c|c|c|c|c|c|c|c|c|c|c|c|c|}
\hline \multirow[b]{2}{*}{ Sampling 1} & \multicolumn{3}{|c|}{ Copper } & \multicolumn{3}{|c|}{ Cadmium } & \multicolumn{3}{|c|}{ Nickel } & \multicolumn{3}{|c|}{ Zinc } & \multicolumn{3}{|c|}{ LMWOs } \\
\hline & $\begin{array}{l}\text { Rate } \\
\text { Coef } \\
\left(\mathrm{d}^{-1}\right) \\
\end{array}$ & $\begin{array}{c}\text { Removal } \\
(\%)\end{array}$ & $\begin{array}{l}\text { Outflow } \\
\text { Conc. } \\
(\mathrm{mg} / \mathrm{L})\end{array}$ & $\begin{array}{l}\text { Rate } \\
\text { Coef } \\
\left(\mathrm{d}^{-1}\right) \\
\end{array}$ & $\begin{array}{c}\text { Rem- } \\
\text { oval } \\
(\%) \\
\end{array}$ & $\begin{array}{l}\text { Outflow } \\
\text { Conc. } \\
(\mathrm{mg} / \mathrm{L})\end{array}$ & $\begin{array}{l}\text { Rate } \\
\text { Coef } \\
\left(\mathrm{d}^{-1}\right) \\
\end{array}$ & $\begin{array}{c}\text { Rem- } \\
\text { oval } \\
(\%) \\
\end{array}$ & $\begin{array}{l}\text { Outflow } \\
\text { Conc. } \\
(\mathrm{mg} / \mathrm{L})\end{array}$ & $\begin{array}{l}\text { Rate } \\
\text { Coef } \\
\left(\mathrm{d}^{-1}\right) \\
\end{array}$ & $\begin{array}{c}\text { Rem- } \\
\text { oval } \\
(\%) \\
\end{array}$ & $\begin{array}{l}\text { Outflow } \\
\text { Conc. } \\
(\mathrm{mg} / \mathrm{L})\end{array}$ & $\begin{array}{l}\text { Rate } \\
\text { Coef } \\
\left(\mathrm{d}^{-1}\right) \\
\end{array}$ & $\begin{array}{c}\text { Removal } \\
(\%)\end{array}$ & $\begin{array}{l}\text { Outflow } \\
\text { Conc. } \\
(\mathrm{mg} / \mathrm{L})\end{array}$ \\
\hline Series 1 & 0.007 & $50.5 \%$ & 1.98 & 0.005 & $38.9 \%$ & 3.6 & 0.009 & $55.8 \%$ & 2.74 & 0.004 & $32.8 \%$ & 4.1 & 0.082 & $99.9 \%$ & bdl \\
\hline Series 2 & 0.005 & $39.3 \%$ & 2.43 & 0.005 & $36.4 \%$ & 3.75 & 0.0007 & $6.5 \%$ & 5.8 & 0.003 & $26.2 \%$ & 4.5 & 0.082 & $99.9 \%$ & bdl \\
\hline \multicolumn{16}{|l|}{ Sampling 2} \\
\hline Series 1 & 0.047 & $98.9 \%$ & 0.04 & 0.019 & $84.7 \%$ & 0.82 & 0.013 & $71.5 \%$ & 1.87 & 0.014 & $73.7 \%$ & 1.52 & 0.082 & $99.9 \%$ & bdl \\
\hline Series 2 & 0.044 & $98.6 \%$ & 0.05 & 0.02 & $85.2 \%$ & 0.79 & 0.015 & $75.6 \%$ & 1.6 & 0.014 & $73.9 \%$ & 1.51 & 0.082 & $99.9 \%$ & bdl \\
\hline \multicolumn{16}{|l|}{ Sampling 3} \\
\hline Series 1 & 0.011 & $63.8 \%$ & 0.54 & 0.029 & $94.3 \%$ & 0.25 & 0.02 & $85.7 \%$ & 0.61 & 0.014 & $73.3 \%$ & 1.22 & 0.082 & $99.9 \%$ & bdl \\
\hline Series 2 & 0.033 & $95.9 \%$ & 0.06 & 0.04 & $97.9 \%$ & 0.09 & 0.04 & $97.9 \%$ & 0.09 & 0.064 & $99.8 \%$ & 0.01 & 0.082 & $99.9 \%$ & bdl \\
\hline \multicolumn{16}{|l|}{ Sampling 4} \\
\hline Series 1 & 0.009 & $57.5 \%$ & 1.49 & 0.008 & $53.0 \%$ & 2.09 & 0.014 & $72.8 \%$ & 1.31 & 0.022 & $87.5 \%$ & 0.44 & 0.082 & $99.9 \%$ & bdl \\
\hline Series 2 & 0.049 & $99.1 \%$ & 0.03 & 0.076 & $99.9 \%$ & $<0.003$ & 0.044 & $98.5 \%$ & 0.07 & 0.039 & $97.7 \%$ & 0.08 & 0.082 & $99.9 \%$ & bdl \\
\hline \multicolumn{16}{|l|}{ Sampling 5} \\
\hline Series 1 & 0.037 & $97.2 \%$ & 0.08 & 0.048 & $98.9 \%$ & 0.02 & 0.02 & $85.8 \%$ & 0.41 & 0.04 & $97.9 \%$ & 0.08 & 0.082 & $99.9 \%$ & bdl \\
\hline Series 2 & 0.052 & $99.3 \%$ & 0.02 & 0.032 & $95.5 \%$ & 0.09 & 0.045 & $98.6 \%$ & 0.04 & 0.055 & $99.5 \%$ & 0.02 & 0.082 & $99.9 \%$ & bdl \\
\hline
\end{tabular}

bdl - below detection limit 
Table 53. Comparisons Between Removal Extent $(\mathrm{mg} / \mathrm{L})$ and Beneficial Use Guidelines and Criteria $(\mathrm{mg} / \mathrm{L})$.

\begin{tabular}{|c|c|c|c|c|c|c|}
\hline & & Copper & Nickel & Zinc & Cadmium & LMWOs \\
\hline \multicolumn{7}{|l|}{$\begin{array}{l}\text { Sampling } \\
1\end{array}$} \\
\hline & Series 1 & 1.98 & 2.74 & 3.9 & 3.6 & $<1.0$ \\
\hline & Series 2 & 2.43 & 5.8 & 4.5 & 3.75 & $<1.0$ \\
\hline \multicolumn{7}{|l|}{$\begin{array}{l}\text { Sampling } \\
2\end{array}$} \\
\hline & Series 1 & 0.04 & 1.87 & 1.52 & 0.82 & $<1.0$ \\
\hline & Series 2 & 0.05 & 1.6 & 1.51 & 0.79 & $<1.0$ \\
\hline \multicolumn{7}{|l|}{$\begin{array}{l}\text { Sampling } \\
3\end{array}$} \\
\hline & Series 1 & 0.54 & 0.61 & 1.22 & 0.25 & $<1.0$ \\
\hline & Series 2 & 0.06 & 0.09 & 0.01 & 0.09 & $<1.0$ \\
\hline \multicolumn{7}{|l|}{$\begin{array}{c}\text { Sampling } \\
4\end{array}$} \\
\hline & Series 1 & 1.49 & 1.31 & 0.44 & 2.09 & $<1.0$ \\
\hline & Series 2 & 0.03 & 0.07 & 0.08 & $<0.003$ & $<1.0$ \\
\hline \multicolumn{7}{|l|}{$\underset{5}{\text { Sampling }}$} \\
\hline & Series 1 & 0.084 & 0.41 & 0.08 & 0.02 & $<1.0$ \\
\hline & Series 2 & 0.02 & 0.04 & 0.02 & 0.09 & $<1.0$ \\
\hline \multicolumn{2}{|c|}{ Irrigation Guidelines } & 0.2 & 0.2 & 2 & 0.01 & 35 \\
\hline \multicolumn{2}{|c|}{$\begin{array}{l}\text { Livestock Guidelines } \\
\text { Surface Discharge }\end{array}$} & 0.5 & - & 24 & 0.05 & 35 \\
\hline & & $0.37-1.0$ & 2 & $0.4-5$ & - & 15 \\
\hline
\end{tabular}


Table 54. Selenium and arsenic removal extents, efficiencies, and rate coefficients for all sampling periods.

\begin{tabular}{|c|c|c|c|c|c|c|c|}
\hline \multirow[b]{2}{*}{ Sampling Period } & \multirow[b]{2}{*}{ Parameters } & \multicolumn{3}{|c|}{ Selenium } & \multicolumn{3}{|c|}{ Arsenic } \\
\hline & & $\begin{array}{l}\text { Conc. } \\
(\mu \mathrm{g} / \mathrm{L})\end{array}$ & $\begin{array}{c}\text { Removal } \\
(\%)\end{array}$ & $\begin{array}{c}\text { Rate } \\
\text { Coef. } \\
\left(\mathrm{d}^{-1}\right)\end{array}$ & $\begin{array}{l}\text { Conc. } \\
(\mu \mathrm{g} / \mathrm{L})\end{array}$ & $\begin{array}{c}\text { Removal } \\
(\%)\end{array}$ & $\begin{array}{l}\text { Rate Coef. } \\
\qquad\left(d^{-1}\right)\end{array}$ \\
\hline \multirow{4}{*}{ 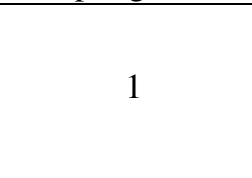 } & Influent & 44.6 & -- & -- & 21.8 & -- & -- \\
\hline & Control Extent & 45.7 & -2.5 & -0.006 & 5.6 & 74.3 & 0.340 \\
\hline & AquaSmart ${ }^{\mathrm{TM}}$ Extent & nd & $97.8^{1}$ & 1.27 & 3.3 & 84.9 & 0.472 \\
\hline & Sucrose Extent & 6.1 & 86.3 & 0.497 & 13.4 & 38.5 & 0.122 \\
\hline \multirow{4}{*}{2} & Influent & 49.7 & -- & -- & 14.0 & -- & -- \\
\hline & Control Extent & 44.6 & 10.3 & 0.027 & 5.0 & 64.3 & 0.257 \\
\hline & AquaSmart ${ }^{\mathrm{TM}}$ Extent & nd & 98.0 & 0.977 & 6.3 & 55.0 & 0.200 \\
\hline & Sucrose Extent & 8.1 & 83.7 & 0.454 & 5.5 & 60.7 & 0.234 \\
\hline \multirow{4}{*}{3} & Influent & 46.5 & -- & -- & 18.4 & -- & -- \\
\hline & Control Extent & 37.9 & 18.5 & 0.051 & 10.9 & 40.8 & 0.131 \\
\hline & AquaSmart ${ }^{\mathrm{TM}}$ Extent & 1.0 & $97.8^{1}$ & 1.28 & 7.7 & 58.2 & 0.218 \\
\hline & Sucrose Extent & 7.3 & 84.3 & 0.463 & 5.1 & 72.3 & 0.321 \\
\hline \multirow{4}{*}{4} & Influent & 42.7 & -- & -- & 18.4 & -- & -- \\
\hline & Control Extent & 39.7 & 7.0 & 0.018 & 6.0 & 67.4 & 0.280 \\
\hline & AquaSmart ${ }^{\mathrm{TM}}$ Extent & 1.0 & $97.7^{1}$ & 1.25 & 9.0 & 51.1 & 0.179 \\
\hline & Sucrose Extent & 1.0 & 97.7 & 0.939 & 6.3 & 65.8 & 0.268 \\
\hline \multirow{4}{*}{5} & Influent & 76.0 & -- & -- & 18.3 & -- & -- \\
\hline & Control Extent & 58.2 & 23.4 & 0.067 & 4.3 & 76.5 & 0.362 \\
\hline & AquaSmart ${ }^{\mathrm{TM}}$ Extent & 1.0 & $98.7^{2}$ & 2.17 & 9.7 & 47.0 & 0.159 \\
\hline & Sucrose Extent & 5.8 & 92.4 & 0.643 & 3.5 & 80.9 & 0.414 \\
\hline \multirow{4}{*}{6} & Influent & 50.8 & -- & -- & 17.2 & -- & -- \\
\hline & Control Extent & 56.4 & -11.0 & -0.026 & 2.4 & 86.0 & 0.492 \\
\hline & AquaSmart ${ }^{\mathrm{TM}}$ Extent & 1.0 & 98.0 & 0.982 & 6.9 & 59.9 & 0.228 \\
\hline & Sucrose Extent & 2.9 & 94.3 & 0.716 & 9.8 & 43.0 & 0.141 \\
\hline
\end{tabular}

${ }^{1}$ Rate coefficient calculated for 3 days of treatment since concentrations in cells 3 and 4 were non-detectable. The rate coefficient was calculated for the HRT during which removal was measured.

${ }^{2}$ Rate coefficient calculated for 2 days of treatment since concentrations in cells 2, 3, and 4 were non-detectable. The rate coefficient was calculated for the HRT during which removal was measured. 
Table 55. Metal concentrations measured in CWTS influent, removal efficiencies (\%), and removal rate coefficients $\left(\mathrm{d}^{-1}\right)$. Removal efficiences and removal rate coefficients were calculated using measured concentrations in the effluent (4-day HRT).

\begin{tabular}{|c|c|c|c|c|c|c|c|c|c|c|c|c|c|}
\hline & & \multicolumn{3}{|c|}{ Iron } & \multicolumn{3}{|c|}{ Manganese } & \multicolumn{3}{|c|}{ Nickel } & \multicolumn{3}{|c|}{ Zinc } \\
\hline & & $\begin{array}{l}\text { Conc. } \\
(\mu \mathrm{g} / \mathrm{L})\end{array}$ & $\begin{array}{c}\text { Removal } \\
(\%)\end{array}$ & $\begin{array}{c}\text { Rate Coef. } \\
\left(\mathrm{d}^{-1}\right)\end{array}$ & $\begin{array}{l}\text { Conc. } \\
(\mu \mathrm{g} / \mathrm{L})\end{array}$ & $\begin{array}{c}\text { Removal } \\
(\%)\end{array}$ & $\begin{array}{c}\text { Rate Coef. } \\
\left(\mathrm{d}^{-1}\right)\end{array}$ & $\begin{array}{l}\text { Conc. } \\
(\mu \mathrm{g} / \mathrm{L})\end{array}$ & $\begin{array}{c}\text { Removal } \\
(\%)\end{array}$ & $\begin{array}{c}\text { Rate Coef. } \\
\left(\mathrm{d}^{-1}\right)\end{array}$ & $\begin{array}{l}\text { Conc. } \\
(\mu \mathrm{g} / \mathrm{L})\end{array}$ & $\begin{array}{c}\text { Removal } \\
(\%)\end{array}$ & $\begin{array}{c}\text { Rate } \\
\text { Coef. }\left(\mathrm{d}^{-1}\right) \\
\end{array}$ \\
\hline \multirow{4}{*}{ 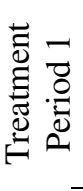 } & Influent & 547.0 & -- & -- & 480.7 & -- & -- & 346.7 & -- & -- & 6480 & -- & -- \\
\hline & FWS Effluent & 58.8 & 89.3 & 0.523 & 16.1 & 96.7 & 0.827 & 266.7 & 23.1 & 0.064 & 1040 & 84.0 & 0.463 \\
\hline & SSF1 Effluent & 16.7 & 96.9 & 0.809 & 2.9 & 99.4 & 1.13 & 116.7 & 66.3 & 0.269 & 23 & 99.6 & 1.37 \\
\hline & SSF2 Effluent & 14.5 & 97.3 & 0.816 & 2.4 & 99.5 & 1.15 & 117.9 & 66.0 & 0.269 & 29 & 99.6 & 1.28 \\
\hline \multirow{4}{*}{ 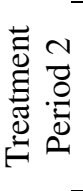 } & Influent & 296.5 & -- & -- & 409.6 & -- & -- & 312.8 & -- & -- & 4740 & -- & -- \\
\hline & FWS Effluent & 71.7 & 75.8 & 0.364 & 32.4 & 92.1 & 0.631 & 180.7 & 42.2 & 0.120 & 2560 & 46.0 & 0.135 \\
\hline & SSF1 Effluent & 10.9 & 96.3 & 0.696 & 3.0 & 99.3 & 0.986 & 109.0 & 65.2 & 0.260 & 791 & 83.3 & 0.441 \\
\hline & SSF2 Effluent & 13.3 & 95.5 & 0.680 & 2.7 & 99.3 & 1.03 & 112.1 & 64.2 & 0.240 & 955 & 79.9 & 0.377 \\
\hline \multirow{4}{*}{ 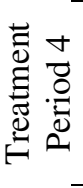 } & Influent & 286.7 & -- & -- & 510.2 & -- & -- & 322.2 & -- & -- & 5230 & -- & -- \\
\hline & FWS Effluent & 64.4 & 77.5 & 0.359 & 31.1 & 93.9 & 0.677 & 255.2 & 20.8 & 0.060 & 1010 & 80.7 & 0.418 \\
\hline & SSF1 Effluent & 15.2 & 94.7 & 0.693 & 4.3 & 99.2 & 0.996 & 139.2 & 56.8 & 0.204 & 289 & 94.5 & 0.649 \\
\hline & SSF2 Effluent & 12.1 & 95.8 & 0.701 & 3.1 & 99.4 & 1.08 & 138.2 & 57.1 & 0.213 & 111 & 97.9 & 0.942 \\
\hline \multirow{4}{*}{ 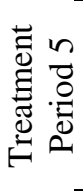 } & Influent & 320.4 & -- & -- & 463.2 & -- & -- & 367.9 & -- & -- & 6230 & -- & -- \\
\hline & FWS Effluent & 73.2 & 77.2 & 0.356 & 50.2 & 89.2 & 0.546 & 191.3 & 48.0 & 0.158 & 1970 & 68.4 & 0.286 \\
\hline & SSF1 Effluent & 12.2 & 96.2 & 0.825 & 4.7 & 99.0 & 0.922 & 129.2 & 64.9 & 0.264 & 587 & 90.6 & 0.521 \\
\hline & SSF2 Effluent & 10.4 & 96.8 & 0.785 & 2.1 & 99.5 & 1.15 & 135.1 & 63.3 & 0.240 & 619 & 90.1 & 0.528 \\
\hline
\end{tabular}


Table 56. Total O \& G concentrations measured in CWTS influent, removal efficiencies (\%), and removal rate coefficients $\left(\mathrm{d}^{-1}\right)$. Removal efficiencies and removal rate coefficients were calculated using measured concentrations in the effluent (4-day HRT).

\begin{tabular}{|c|c|c|c|c|}
\hline & \multicolumn{3}{|c|}{ Oil and Grease } \\
\hline & & Conc. (mg/L) & Removal (\%) & Rate Coef. $\left(\mathrm{d}^{-1}\right)$ \\
\hline \multirow{4}{*}{ 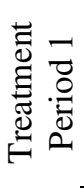 } & Influent & 97.6 & -- & -- \\
\hline & FWS Effluent & 3.2 & 96.7 & 0.771 \\
\hline & SSF1 Effluent & 4.2 & 95.7 & 0.738 \\
\hline & SSF2 Effluent & 3.8 & 96.1 & 0.769 \\
\hline \multirow{4}{*}{ 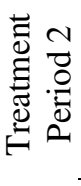 } & Influent & 94.2 & -- & -- \\
\hline & FWS Effluent & 2.3 & 97.6 & 0.809 \\
\hline & SSF1 Effluent & 5.9 & 93.7 & 0.660 \\
\hline & SSF2 Effluent & 4.7 & 95.0 & 0.645 \\
\hline \multirow{4}{*}{ 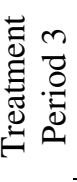 } & Influent & 52.3 & -- & -- \\
\hline & FWS Effluent & 2.2 & 95.8 & 0.735 \\
\hline & SSF1 Effluent & 2.1 & 96.0 & 0.690 \\
\hline & SSF2 Effluent & 2.9 & 94.5 & 0.681 \\
\hline \multirow{4}{*}{ 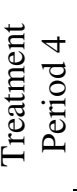 } & Influent & 101.2 & -- & -- \\
\hline & FWS Effluent & 7.1 & 93.0 & 0.650 \\
\hline & SSF1 Effluent & 5.6 & 94.5 & 0.672 \\
\hline & SSF2 Effluent & 4.2 & 95.8 & 0.748 \\
\hline \multirow{4}{*}{ 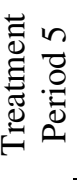 } & Influent & 95.9 & -- & -- \\
\hline & FWS Effluent & 4.2 & 95.6 & 0.729 \\
\hline & SSF1 Effluent & 9.9 & 89.7 & 0.508 \\
\hline & SSF2 Effluent & 7.2 & 92.5 & 0.550 \\
\hline \multirow{4}{*}{ 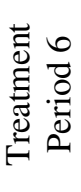 } & Influent & 99.7 & -- & -- \\
\hline & FWS Effluent & 2.9 & 97.1 & 0.783 \\
\hline & SSF1 Effluent & 3.1 & 96.9 & 0.773 \\
\hline & SSF2 Effluent & 5.3 & 94.7 & 0.657 \\
\hline
\end{tabular}


Design and Construct Demonstration Constructed Wetland Treatment System (Task 7)

Specific experiments in support of designing a demonstration system were performed to gain a better understanding of conditions affecting treatment pathways and hybrid design strategies that can be incorporated into constructed wetland treatment. These experiments provide insight into potential design strategies that may be used for the construction of demonstration and full-scale systems. The experiments, which are discussed below, are:

1. Addition of zeolite for sorption of ammonia from produced water;

2. Effects of biogeochemical conditions and processes on vertical distribution of metals in hydrosoil;

3. Effects of water depth on conditions and treatment performance;

4. Effects of seasonal variation on treatment performance;

5. Addition of a nutrient amendment for removal of selenium from produced water;

6. Addition of an oil/water separator for removal of oil and grease; and

7. Evapotranspiration experiments and tracer tests.

\section{Sorption Experiments}

Sorptive materials may be used to remove dissolved ammonia from water. Transfer from the aqueous phase to binding sites may enhance performance in constructed wetland treatment systems (CWTSs) by concentrating ammonia where nitrifying bacteria (Nitrosomonas spp. and Nitrobacter spp.) may be active. The zeolite clinoptilolite was selected as a sorptive medium for use in CWTSs based on its affinity for ammonia and high surface area for growth and attachment of periphytic biofilms. The objective of this research was to determine the effects of clinoptilolite on ammonia treatment performance in pilot-scale CWTSs.

A serial batch sorption experiment was performed to determine the ammonia ion exchange capacity of clinoptilolite. Data from the serial batch sorption experiment were used to predict ammonia removal in response to clinoptilolite added (1000 and $500 \mathrm{~g}$ ) to a pair of pilot-scale CWTSs containing sandy sediment. After 50 days, nitrifying bacterial activity reactivity tests (n-BARTs) were used to detect the presence of nitrifying bacteria in the added clinoptilolite and sandy sediment.

Results indicate that clinoptilolite has an affinity for ammonia described by the Freundlich equation $\mathrm{Q}=0.72 \mathrm{C}_{\mathrm{e}}^{0.57}$ for equilibrium ammonia-N concentrations from 0.07 to $30.1 \mathrm{mg} / \mathrm{L}$ (Table 57 and Figure 29). Addition of 1000g clinoptilolite to a pilot-scale CWTS operating on a 2-day hydraulic retention time (HRT) and containing Schoenoplectus californicus produced a measurable decrease in the outflow concentration of ammonia- $\mathrm{N}$ when compared with a system containing no clinoptilolite ( 2.0 vs. $5.6 \mathrm{mg} / \mathrm{L})$ during a 10-day sampling period (Figure 30). Addition of 500g clinoptilolite to a CWTS operating on 4-day HRT and containing Typha latifolia produced no discernable difference in the outflow concentration of ammonia compared with a system containing no clinoptilolite (Figure 31). n-BARTs indicated similar levels of activity in both the sediment and clinoptilolite in the Schoenoplectus system. No activity was detected in either the sediment or clinoptilolite in the Typha system. The difference in performance between the Schoenoplectus and Typha 
systems was attributed to factors including dissolved oxygen and equilibrium ammonia concentrations. This work demonstrates that clinoptilolite can be effective for increasing ammonia removal and nitrifying activity in pilot-scale CWTSs.

Table 57. Resultant equilibrium concentrations $\left(C_{e}\right)$ and calculated sorption values $(q)$ from sealed $300 \mathrm{~mL}$ BOD bottles containing ammonia solution $\left(\mathrm{C}_{\mathrm{o}}\right)$ and clinoptilolite $\left(\mathrm{M}_{\mathrm{c}}\right)$.

\begin{tabular}{ccccccc}
\hline Bottle & $\mathrm{C}_{\mathrm{o}}(\mathrm{mg} / \mathrm{L})$ & $\mathrm{C}_{\mathrm{e}}(\mathrm{mg} / \mathrm{L})$ & $\mathrm{M}_{\mathrm{c}}(\mathrm{g})$ & $\mathrm{q}(\mathrm{mg} / \mathrm{g})$ & $\log \mathrm{C}_{\mathrm{e}}$ & $\log \mathrm{q}$ \\
\hline 1 & 1 & $<0.1$ & 3.00 & 0.09 & -1.00 & -1.05 \\
2 & 1 & $<0.1$ & 3.00 & 0.09 & -1.00 & -1.05 \\
3 & 2 & 0.21 & 3.00 & 0.18 & -0.68 & -0.75 \\
4 & 2 & 0.22 & 3.00 & 0.18 & -0.66 & -0.75 \\
5 & 5 & 0.59 & 3.00 & 0.44 & -0.23 & -0.36 \\
6 & 5 & 0.63 & 3.00 & 0.44 & -0.20 & -0.36 \\
7 & 10 & 1.38 & 3.00 & 0.86 & 0.14 & -0.06 \\
8 & 10 & 1.42 & 3.00 & 0.86 & 0.15 & -0.07 \\
9 & 20 & 3.65 & 3.00 & 1.63 & 0.56 & 0.21 \\
10 & 20 & 3.89 & 3.00 & 1.61 & 0.59 & 0.21 \\
14 & 40 & 10.9 & 3.00 & 2.91 & 1.04 & 0.46 \\
15 & 40 & 10.8 & 3.00 & 2.92 & 1.03 & 0.47 \\
16 & 80 & 30.1 & 3.00 & 4.99 & 1.48 & 0.70 \\
17 & 80 & 29.7 & 3.00 & 5.03 & 1.47 & 0.70 \\
\hline
\end{tabular}

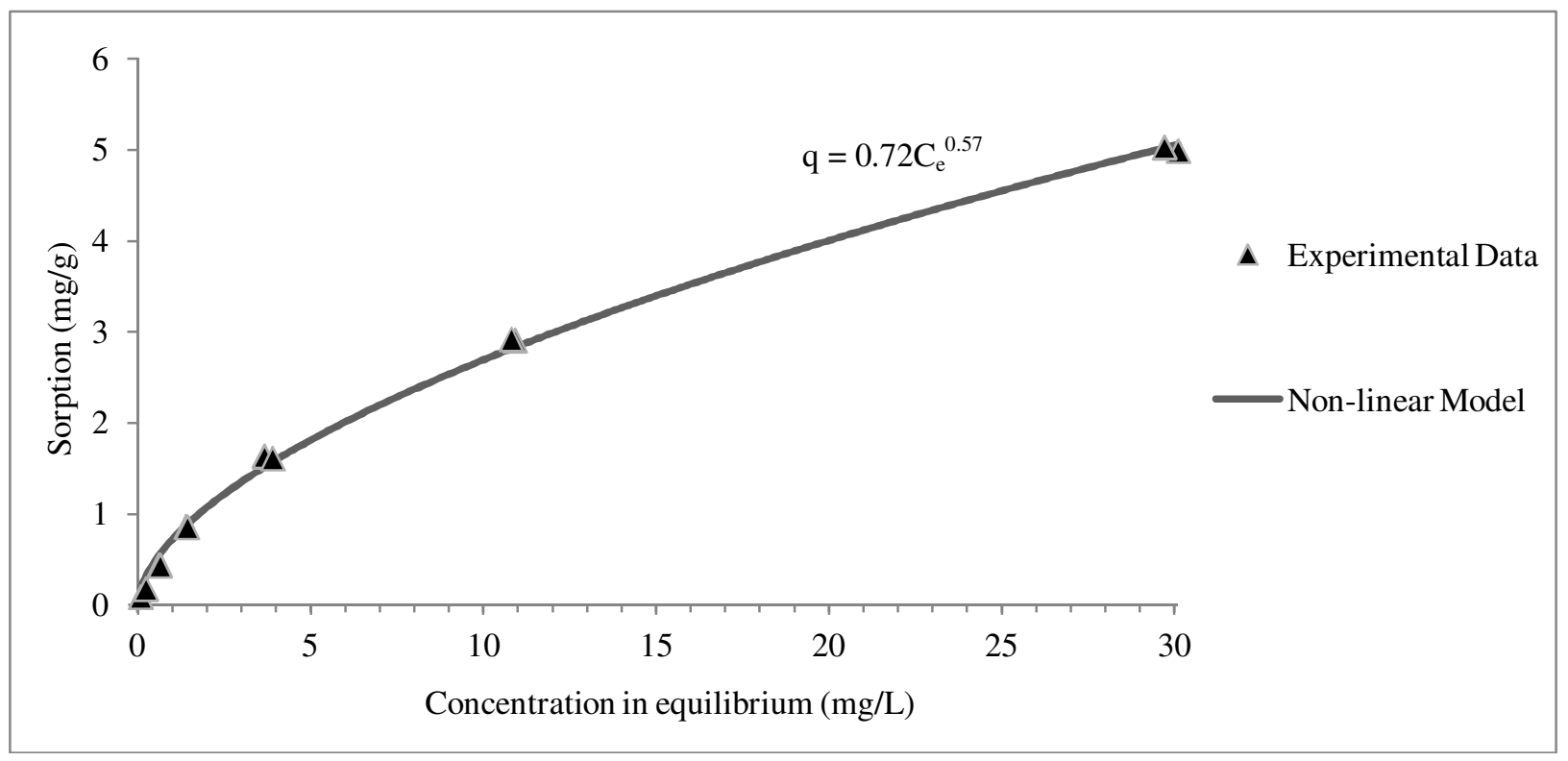

Figure 29. Non-linearly derived Freundlich sorption isotherms generated from serial batch sorption experiment (Table 57). 


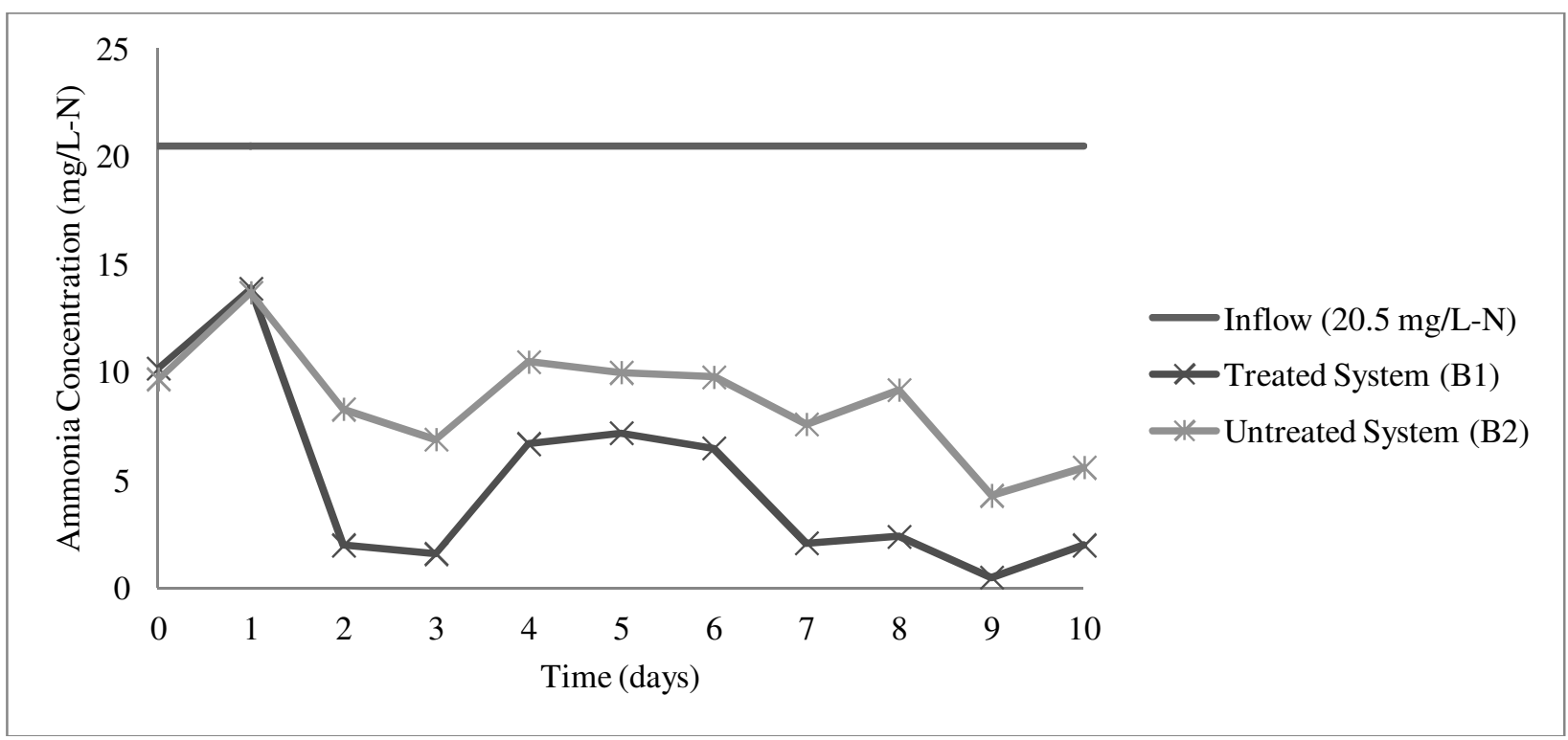

Figure 30. Comparison of outflows from treated and untreated (B1 and B2)

Schoenoplectus systems during the 10-day monitoring period.

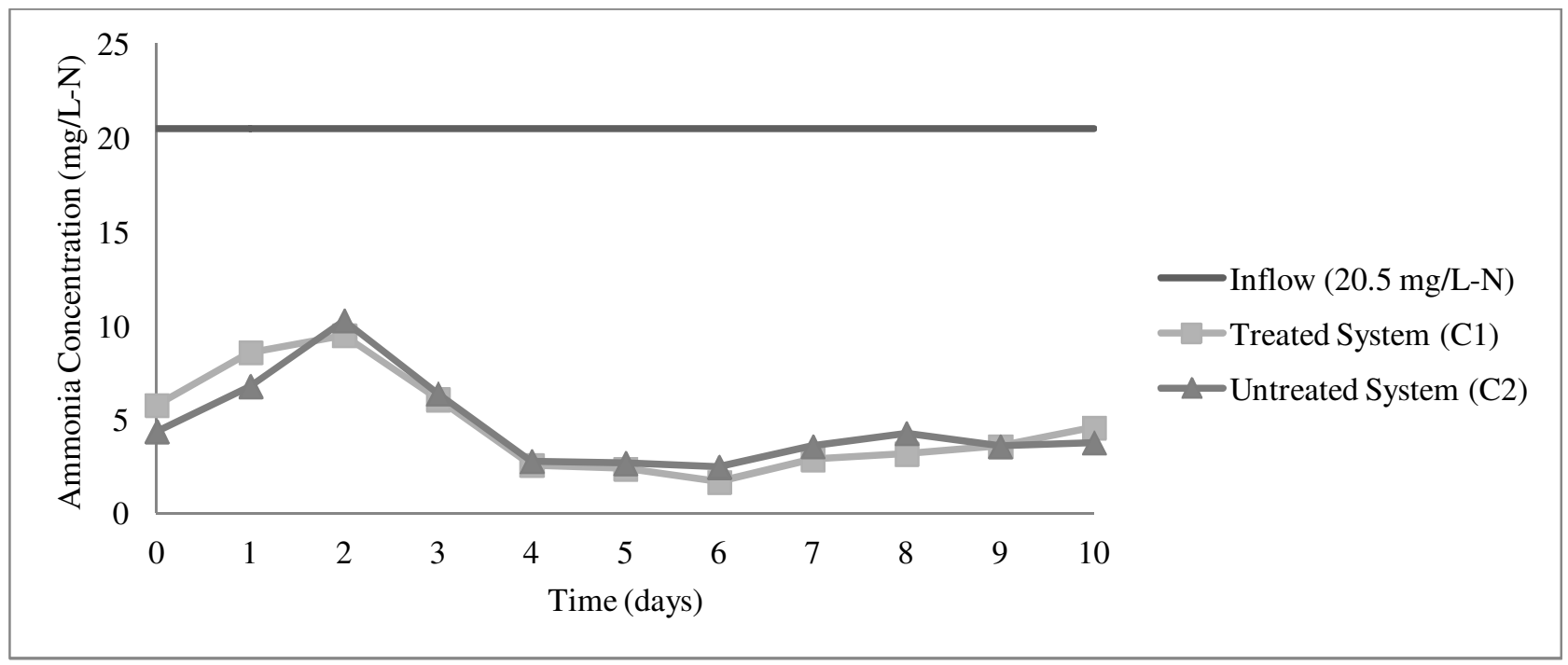

Figure 31. Comparison of outflows from treated and untreated (C1 and C2) Typha systems during the 10-day monitoring period.

\section{Biogeochemical Conditions and Processes}

Environmental conditions in hydrosoil (sediment and overlying organic detritus) of a free water surface pilot-scale constructed wetland treatment system (CWTS) were investigated to determine their effects on sequestering $\mathrm{Cd}, \mathrm{Cu}, \mathrm{Ni}$, and $\mathrm{Zn}$. Redox potential, $\mathrm{pH}$, organic matter content (OM), acid-volatile sulfide (AVS) concentration, simultaneously extracted metal (SEM) concentration, and carbonate content were measured in detritus grab samples and sediment cores extracted from two cells planted with Schoenoplectus californicus (cells 1 and 2) and two cells planted with Typha angustifolia. 
The detritus consisted of an organic-rich layer (i.e. plant roots and plant litter) approximately 16 to $26 \mathrm{~cm}$ thick (Figure 32). The detritus-sediment interface was recognized by a distinct boundary at which dark, organic-rich detritus sharply overlaid lighter mineral-rich sediment. OM was $30-85 \%$ in the detritus, decreasing gradually downward to $0.65-3 \%$ at $15 \mathrm{~cm}$ below the top of the sediment (Figure 32). Reducing conditions (-249 to $-34 \mathrm{mV}$ ) and $\mathrm{pH}$ between 5.01 and 7.41 were measured in the detritus and sediment of all four cells (Figure 32). Highest redox potential (-145 to $-34 \mathrm{mV}$ ) and $\mathrm{pH}$ (6.28 to 7.41) occurred in the upper $6 \mathrm{~cm}$ of sediment. The greatest AVS concentration (36-160 $\mu$ mole/g) and greatest total SEM concentration $(\mathrm{Cd}, \mathrm{Cu}, \mathrm{Ni}$, and $\mathrm{Zn} \geq 5.45$ $\mu$ mole/g) occurred in the detritus.

Measured values of redox potential, $\mathrm{pH}, \mathrm{OM}$, and AVS concentration in CWTS cells were in favorable ranges to promote sorption and complexation of $\mathrm{Cd}, \mathrm{Cu}, \mathrm{Ni}$, and $\mathrm{Zn}$ with AVS (Table 58). Reducing conditions and acidic to circumneutral $\mathrm{pH}$ in the detritus and sediment are interpreted to have resulted from organic matter degradation, nutrient uptake by roots, and microbial processes. Hydrosoil amendments (hay and gypsum) to cells 1 and 2 promoted metal complexation with AVS in the sediment, which resulted in greater mean AVS and $\sum$ SEM concentrations in sediment of cells 1 and 2 (planted with $S$. californicus) compared to cells 3 and 4 (planted with T. angustifolia).

Sequential extractions (after Tessier et al., 1979) and mineralogical analyses using a scanning electron microscopy (EM) equipped with energy dispersion spectroscopy (EDS) were performed on detritus and sediment samples to determine metal mobility and treatment processes in the system. The sequential extraction procedure (SEP) released metals into five operationally defined geochemical fractions: exchangeable; bound to carbonates; bound to Fe-Mn oxides, bound to organic matter or sulfides; and residual.

Metal concentrations in each fraction in the detritus and sediment are shown in Figure 33, and the sum of metal concentrations in each fraction in the detritus and sediment is shown in Figure 34. Metal fractionation and relative (percentage) importance with depth is shown in Figure 35. The fraction of all metals associated with the most mobile fraction (i.e. exchangeable) in each cell was low $(<2 \%$; Figure 34$)$. The high percent of metals associated with the Fe-Mn oxide (i.e. Fe-Mn hydroxide) fraction (22-42\%) indicates oxidation and hydrolysis (Figure 34). OM and concentrations of $\mathrm{Cd}, \mathrm{Cu}, \mathrm{Ni}$, and $\mathrm{Zn}$ decreasing with depth in the hydrosoil of each cell are consistent with sorption to organic matter (Figure 32). The high percent of metals bound to sulfides (39-46\%) confirmed by chemical extractions (SEP and AVS-SEM; Figure 32-35) and mineralogical analysis (scanning EM-EDS) indicates precipitation in the form of sulfide minerals. High AVS concentration (5.9-100 $\mu \mathrm{mole} / \mathrm{g})$ indicates that dissimilatory sulfate reduction occurred (Figure 32). 10 to 15\% of all metals were associated with the residual fraction (Figure 34), which is unlikely to be released into the surface water from $\mathrm{pH}$ or redox potential changes. The metal fractionation results suggest that wetlands should be designed with careful consideration of metal fluxes (i.e. biogeochemical processes) with changes in hydrosoil conditions (i.e. redox potential and $\mathrm{pH}$ ). 

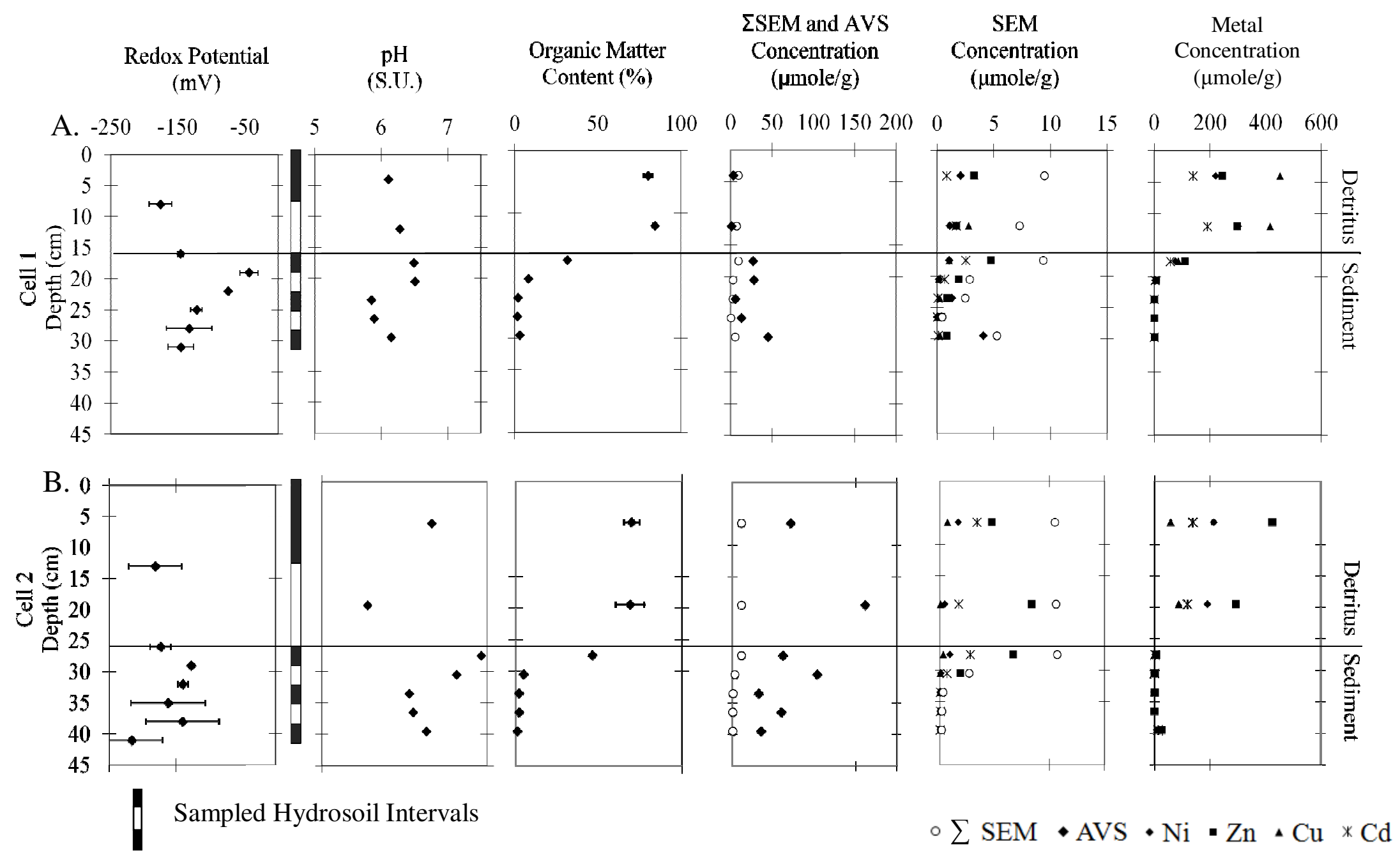

Figure 32 (continued on next page). 

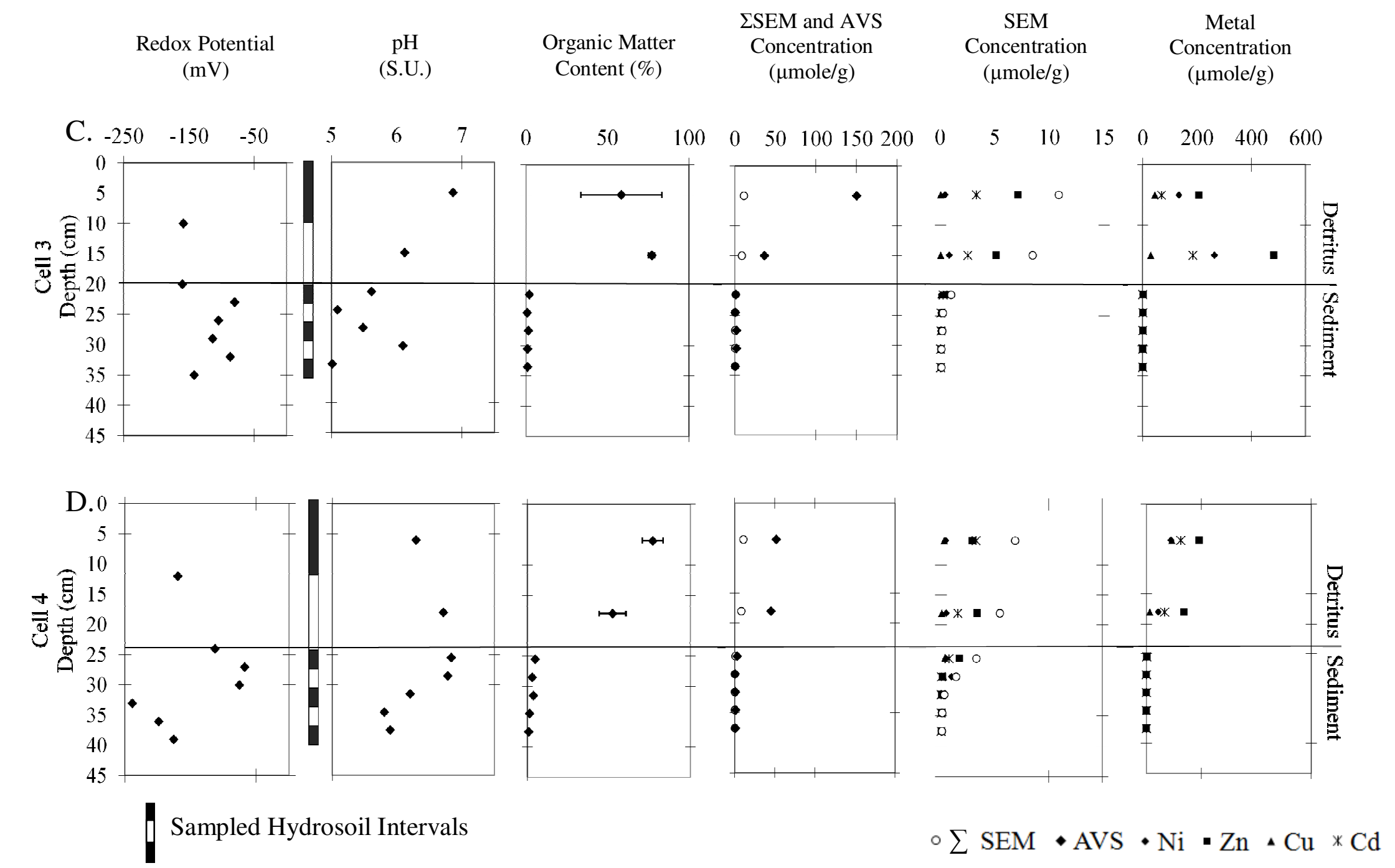

Figure 32 (continued). Vertical (depth) profiles of measured hydrosoil conditions and concentrations of $\mathrm{Cd}, \mathrm{Cu}, \mathrm{Ni}$, and $\mathrm{Zn}$. A. cell 1, B. cell 2, C. cell 3, and D. cell 4. Redox potential was plotted at the specific depths where measured. Seven depth intervals (2 detritus; 5 sediment) in each cell, represented by the shaded bar, were analyzed for $\mathrm{pH}$, organic matter content, $\sum$ SEM concentration, AVS concentration, SEM concentration of $\mathrm{Cd}, \mathrm{Cu}, \mathrm{Ni}$, and $\mathrm{Zn}$, and concentration of $\mathrm{Cd}, \mathrm{Cu}, \mathrm{Ni}$, and $\mathrm{Zn}$ (measurement graphed as a point in the middle of each interval). Zero depth represents the surface water-detritus interface, and the line across the graphs represents the detritus-sediment interface. 
Cell 1

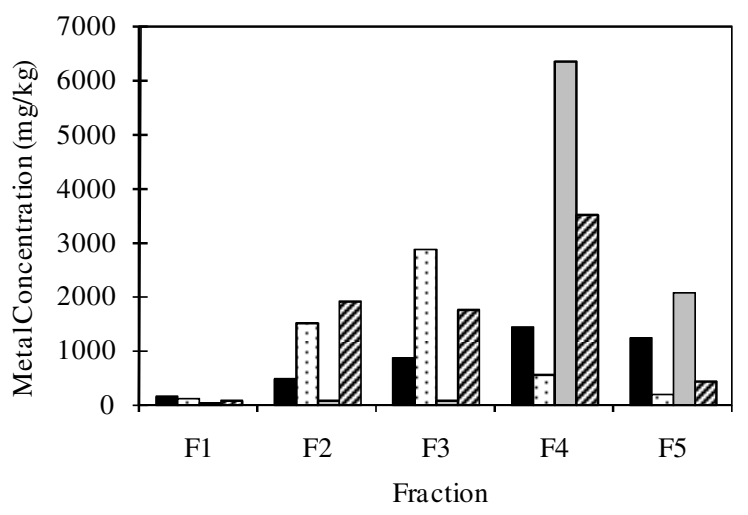

Cell 3

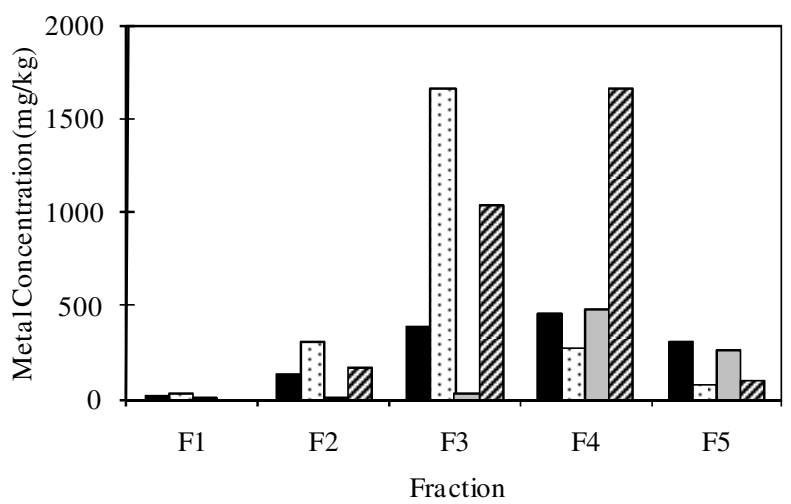

$\square \mathrm{Ni} \quad \square \mathrm{Zn} \quad \square \mathrm{Cu} \quad \square \mathrm{Cd}$

Figure 33. $\mathrm{Ni}, \mathrm{Cu}, \mathrm{Zn}$, and $\mathrm{Cd}$ fractionation in cells 1 and 3 of the pilot-scale CWTS hydrosoil expressed as concentration in $\mathrm{mg} / \mathrm{kg}$ : $\mathrm{F} 1=$ exchangeable; $\mathrm{F} 2=$ bound to carbonates; $\mathrm{F} 3=$ bound to Fe-Mn oxides; F4= bound to organics or sulfides; F5= bound to the residual fraction (Table 3). Fractionation of metals demonstrates changes in concentrations among fractions which a metal occurs in a cell planted with $S$. californicus (cell 1) and a cell planted with $T$. angustifolia (cell $3)$.

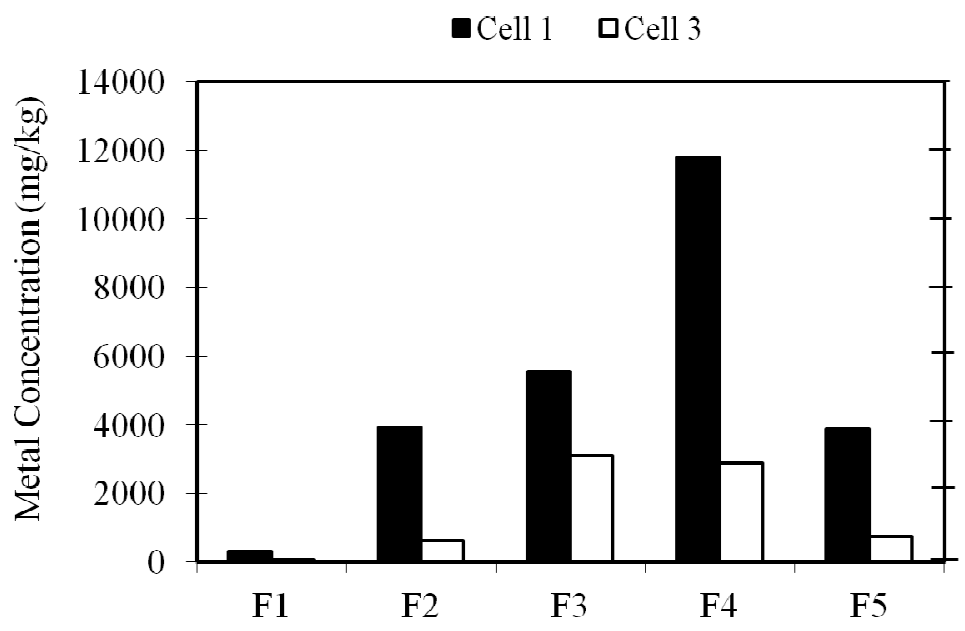

Fraction

Figure 34. Fractionation of all metals (total $\mathrm{Cd}, \mathrm{Cu}, \mathrm{Ni}$, and $\mathrm{Zn}$ ) in cells 1 and 3 of the pilot-scale CWTS expressed as concentration in $\mathrm{mg}$ all metals $/ \mathrm{kg}$ of hydrosoil: $\mathrm{F} 1=$ exchangeable; $\mathrm{F} 2=$ bound to carbonates; F3= bound to Fe-Mn oxides; F4= bound to organics or sulfides; F5= bound to the residual fraction (Table 3). Fractionation of all metals demonstrates changes in concentrations among fractions which all metals measured occur in a cell planted with $S$. californicus (cell 1) and a cell planted with T. angustifolia (cell 3). 
Cell 1

$\mathrm{Ni}$
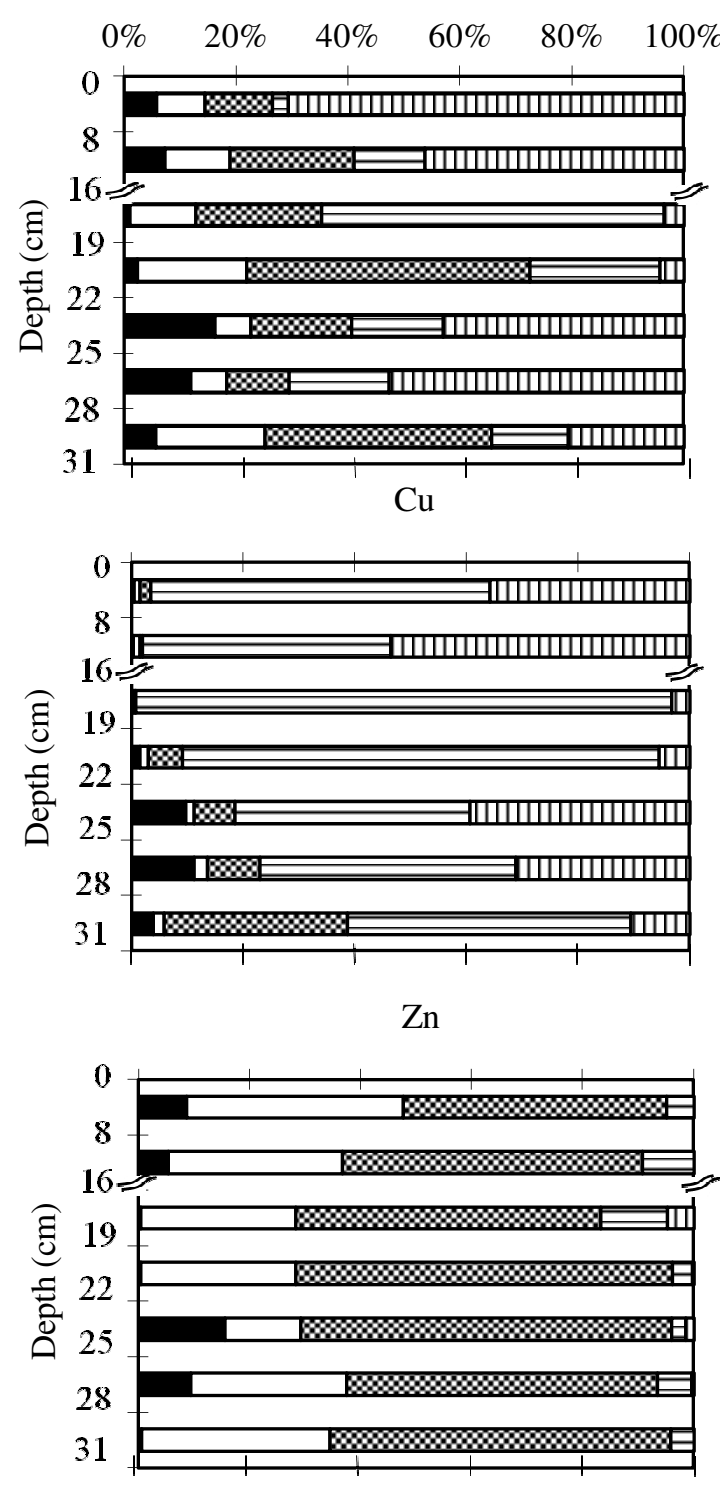

$\mathrm{Cd}$

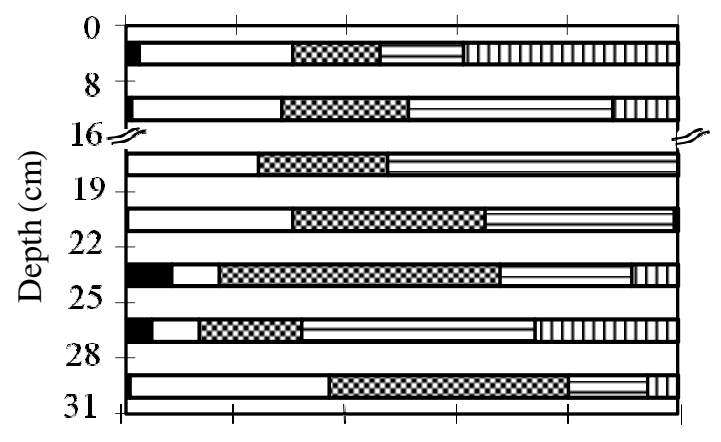

Cell 3

$\mathrm{Ni}$
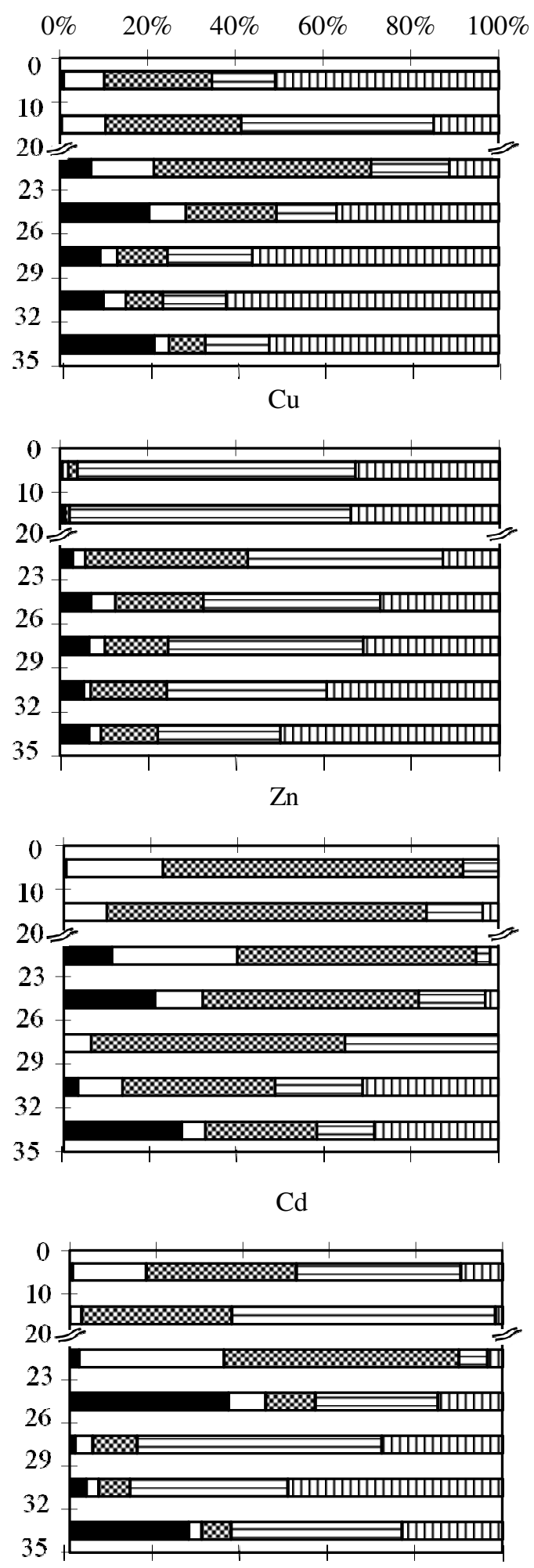

学3 昨4 四F5

Figure 35. $\mathrm{Cd}, \mathrm{Cu}, \mathrm{Ni}$, and $\mathrm{Zn}$ fractionation in cells 1 and 3 of the pilot-scale CWTS expressed as percentages of the total concentrations with depth: $\mathrm{F} 1=$ exchangeable; $\mathrm{F} 2=$ bound to carbonates; $\mathrm{F} 3=$ bound to Fe-Mn oxides; F4= bound to organics or sulfides; F5= bound to the residual fraction. 
Table 58. Comparison of values for hydrosoil conditions favorable for biogeochemical processes and measured values of conditions in detritus and sediment

\begin{tabular}{|c|c|c|c|c|c|}
\hline & \multicolumn{2}{|c|}{ Favorable Values } & \multicolumn{2}{|c|}{ Measured Values } & \multirow[t]{2}{*}{ Summary } \\
\hline & Sorption & Complexation with AVS & Detritus $^{c}$ & Sediment $^{\mathrm{c}}$ & \\
\hline $\begin{array}{l}\text { Redox } \\
\text { Potential } \\
\text { Range }(\mathrm{mV})\end{array}$ & -300 to $700^{a, b}$ & -300 to $0^{\mathrm{a}, \mathrm{b}}$ & -209 to -113 & -249 to -34 & $\begin{array}{l}\text { Redox favorable for } \\
\text { sorption and sulfide } \\
\text { formation in hydrosoil }\end{array}$ \\
\hline $\begin{array}{l}\text { pH Range } \\
\text { (S.U.) }\end{array}$ & $\begin{array}{l}2 \text { to } 10^{\mathrm{a}} \\
\text { Greatest sorption at } \mathrm{pH} \sim 8, \\
\text { sorption decreases as } \mathrm{pH} \\
\text { decreases }^{\mathrm{d}}\end{array}$ & $\begin{array}{l}4 \text { to } 9.5^{\mathrm{f}} \\
\text { Complexation with AVS favored } \\
\text { in basic } \mathrm{pH}^{\mathrm{e}}\end{array}$ & 5.69 to 6.86 & 5.01 to 7.41 & $\begin{array}{l}\mathrm{pH} \text { favorable for sorption } \\
\text { and sulfide formation in } \\
\text { hydrosoil }\end{array}$ \\
\hline $\begin{array}{l}\text { Organic Matter } \\
\text { Content } \\
(\mathrm{OM} ; \%)\end{array}$ & $\begin{array}{l}\text { Sorption increases with } \\
\text { increasing organic content }\end{array}$ & $\begin{array}{l}>0.1 \% \text { dry weight needed for } \\
\text { dissimilatory sulfate reducing } \\
\text { bacteria }^{\mathrm{h}}\end{array}$ & 29.7 to 85.0 & 0.646 to 46.1 & $\begin{array}{l}\text { OM favorable for sorption } \\
\text { and sulfide formation in } \\
\text { hydrosoil }\end{array}$ \\
\hline Vegetation & $\begin{array}{l}\text { Sorption increases with } \\
\text { increasing mass of roots and } \\
\text { shoots }^{i}\end{array}$ & $\begin{array}{l}\text { Complexation with AVS favored } \\
\text { by vegetation with small radial } \\
\text { oxygen loss from root zone }\end{array}$ & $\begin{array}{l}16 \text { to } 26 \mathrm{~cm} \text { thick root } \\
\text { mass comprising } \\
35-46 \% \text { of the hydrosoil }\end{array}$ & $\begin{array}{l}\text { Few thick plant } \\
\text { roots, extend } \\
\text { downward as } \\
\text { plant anchors }\end{array}$ & $\begin{array}{l}\text { Vegetation favorable for } \\
\text { sorption in detritus }\end{array}$ \\
\hline $\begin{array}{l}\text { AVS } \\
\text { concentration } \\
(\mu \text { mole/g) }\end{array}$ & N/A & $\begin{array}{l}\text { Complexation with AVS } \\
\text { increases with increasing AVS } \\
\text { concentration }\end{array}$ & $36-160$ in cells 2 to 4 & $\begin{array}{l}5.9-100 \text { in } \\
\text { cells } 1 \text { and } 2 \\
0.54-3.0 \text { in } \\
\text { cells } 3 \text { and } 4\end{array}$ & $\begin{array}{l}\text { AVS concentration most } \\
\text { favorable for sulfide } \\
\text { formation in detritus of } \\
\text { cells } 2 \text { to } 4 \text { and in } \\
\text { sediment of cells } 1 \text { and } 2\end{array}$ \\
\hline \multicolumn{6}{|c|}{$\begin{array}{l}\text { N/A Not applicable for treatment process or hydrosoil zone } \\
\text { a. Under Conditions of } 25^{\circ} \mathrm{C} \text { and } 1 \text { bar (Brookins, 1988) } \\
\text { b. Typical wetland Eh values (Reddy and DeLaune, 2008) } \\
\text { c. Inflow and outflow values from cells } 1 \text { and 2; Outflow values from cells } 3 \text { and } 4 \\
\text { d. Abolinno et al. (2003); Msaky and Calvet (1990); Oyanedel-Craver and Smith (2006) } \\
\text { e. Patterson et al. (1977) }\end{array}$} \\
\hline
\end{tabular}




\section{Water Depth}

Water depths in free water surface constructed wetland treatment systems (CWTSs) are usually established based on practicalities such as environmental requirements and tolerances of plants, height of water control or containment structures (berms), and periodicity and intensity of rainfall events. The availability of sufficient land at a specific site for the required "footprint" is often another important consideration regarding water depth in constructed wetlands for treatment of contaminated waters. In design of CWTSs, the "footprint" is based on the hydraulic retention time (HRT) necessary to achieve the required treatment performance (Kadlec and Knight, 1996; Rodgers and Castle, 2008). Water depth is a critical factor related to the flow volume, flow rate, and the HRT required for removal of targeted constituents (Kadlec and Knight, 1996). However, the influence of water depth on treatment performance in CWTSs is a relatively unstudied factor (Gillespie et al. 1998; Tanner et al. 1998; Headley et al. 2005; Aguirre et al. 2005).

Some factors impacting performance of free water surface CWTSs that can be influenced by water depth include: 1) net oxygen supply rate (NOSR; Kadlec and Knight, 1996), 2) sediment oxidation-reduction potential (redox; Gambrell et al. 1991), 3) removal pathways and processes for targeted constituents that occur in CWTSs (e.g. metal precipitation and biodegradation; Rodgers and Castle, 2008), 4) plant survival and growth (Brix and Sorrell, 1996; Sorrell et al. 2002), 5) mass loading rates (Tao et al. 2005; Caselles-Osorio and García, 2006), and 6) organic matter content in the sediment (Aguirre et al. 2005). Clearly, these factors can contribute independently and collectively to the treatment performance achieved by a CWTS (Allen et al. 1993; Brix, 1994; Obire and Nwaubeta, 2001; Kadlec and Knight, 1996).

The purpose of this research was to evaluate the influence of a series of water depths on factors affecting treatment performance of pilot-scale CWTSs for renovating simulated FOPW. Specific objectives of this study were to: 1) prepare simulated FOPW, 2) design and construct pilot-scale CWTSs, 3) compare treatment performance data (i.e. rates and extents of removal of $\mathrm{Cd}, \mathrm{Cu}, \mathrm{Ni}, \mathrm{Zn}$, and oil) for the pilot-scale CWTSs with a $36 \mathrm{~h}$ HRT at five incremental water depths (i.e. 15, 23, 33, 46, and $56 \mathrm{~cm}$ ), and 4) measure sediment redox potential and sediment organic matter content in pilot-scale CWTS wetland cells planted with Schoenoplectus californicus and wetland cells containing Typha angustifolia.

Simulated FOPW was formulated based on constituent concentrations in FOPWs reported in peer-reviewed literature (Veil et al. 2004; Thordsen et al. 2007; Alley et al. 2011) and government documents (USEPA, 2000; USGS, 2002) as well as analyses of PW samples from oilfield sites in the continental United States. An ionic balance was calculated (i.e. $\Sigma$ cations $(\mathrm{meq} / \mathrm{L})+\Sigma$ anions $(\mathrm{meq} / \mathrm{L})$ from analyte concentrations in actual FOPW to formulate simulated FOPW with appropriate salts to accurately achieve ionic composition and strength as well as salinity and hardness. The oil component for the simulated FOPW formulation was conventional light oil (Rotella ${ }^{\circledR} \mathrm{T} 15 \mathrm{~W} 40$ ), a cost-effective and reproducible alternative to using an actual crude oil. General water characteristics (i.e. $\mathrm{pH}$, dissolved oxygen, conductivity, alkalinity, and hardness) were also measured to compare simulated and actual FOPW. Simulated FOPW was composed of $\mathrm{Cd}, \mathrm{Cu}, \mathrm{Ni}, \mathrm{Zn}$, oil, and 
salts well-mixed with $3780 \mathrm{~L}$ of municipal water in a reservoir (USEPA, 2000; USGS, 2002; Veil et al. 2004; Thordsen et al. 2007; Alley et al. 2011; Table 1). This formulation of FOPW resulted in the following concentrations of constituents targeted for treatment: 6 $\mathrm{mg} \mathrm{Cd}^{+2} / \mathrm{L}, 4 \mathrm{mg} \mathrm{Cu}^{+2} / \mathrm{L}, 6 \mathrm{mg} \mathrm{Ni}^{+2} / \mathrm{L}, 6 \mathrm{mg} \mathrm{Zn}^{+2} / \mathrm{L}$, and $100 \mathrm{mg}$ Rotella ${ }^{\circledR} \mathrm{T}$ 15W40/L.

The pilot-scale wetlands were designed and constructed with emphasis on water depth as a treatment variable. Design of the pilot-scale systems was premised on the calculated rate of oxygen diffusion through the water column to the sediment of a wetland cell. The film theory of mass transfer and the NOSR equation from Kadlec and Knight (1996) were used to estimate the rate of oxygen supply to the sediment at different water depths to predict sediment redox conditions that could be achieved. Oxygen diffusivity, defined as the ability of oxygen to diffuse through water at a specific depth, was estimated based on the film theory of mass transfer equation:

$$
K=\left[\begin{array}{l}
\mathrm{D} \\
\mathrm{d}
\end{array}\right]
$$

where, $\mathrm{K}$ is the reaeration rate, $\mathrm{m} / \mathrm{d}, \mathrm{D}$ is the molecular diffusivity of oxygen in water, $\mathrm{m}^{2} / \mathrm{d}$, and $\mathrm{d}$ is the water depth, $\mathrm{m}$. Transfer of oxygen from the atmosphere to the sediment in the wetland can be estimated using the NOSR equation (Kadlec and Knight, 1996):

$$
\mathrm{NOSR}=\mathrm{K}\left(\mathrm{C}_{\mathrm{DO}}^{\mathrm{sat}}-\mathrm{C}_{\mathrm{DO}}\right)-\mathrm{SOD}
$$

where NOSR is the net oxygen supply rate, $\mathrm{g} / \mathrm{m}^{2} * \mathrm{~d}, \mathrm{~K}$ is the reaeration coefficient, $\mathrm{m} / \mathrm{d}$ (estimated from the film theory of mass transfer equation), $\mathrm{C}^{\text {sat }}$ DO is the saturated dissolved oxygen concentration at $1 \mathrm{~atm}, 8.7 \mathrm{mg} \mathrm{O} / 2 / \mathrm{L}\left(\mathrm{g} / \mathrm{m}^{3}\right.$; APHA, 2005; Huddleston et al. 2008), $\mathrm{C}_{\mathrm{DO}}$ is the average dissolved oxygen concentration in the bulk of water, $8.0 \mathrm{mg} \mathrm{O} / \mathrm{L}\left(\mathrm{g} / \mathrm{m}^{3}\right.$; Huddleston et al. 2008), and SOD is sediment oxygen demand, $0.1 \pm 0.01 \mathrm{mg} \mathrm{O}_{2} /$ day (Huddleston et al. 2008). We assumed that the SOD value from Huddleston et al. (2008) will estimate the SOD for these wetland cells. Huddleston et al. (2008) measured SOD using the same sediment and comparable formulation and loadings of Osmocote ${ }^{\circledR}$ as in this experiment with a water depth of $30 \mathrm{~cm}$. A general equation was formulated relating water depth to HRT for these specific pilot-scale CWTSs:

$$
\mathrm{d}=\frac{\mathrm{HRT}^{*} \mathrm{Q}}{\pi \mathrm{r}^{2}}
$$

where, $\mathrm{d}$ is the water depth, $\mathrm{cm}, \mathrm{HRT}$ is the hydraulic retention time, min, $\mathrm{Q}$ is flow rate, $\mathrm{ml} / \mathrm{min}$, and, $\pi \mathrm{r}^{2}$ is the surface area of the wetland, $\mathrm{cm}^{2}$. The following equation determines the mass loading rates for each wetland cell and series:

$$
\mathrm{ML}=\mathrm{C} * \mathrm{R}
$$

where, $\mathrm{ML}$ is the mass loading rate, $\mathrm{mg} / \mathrm{min}, \mathrm{C}$ is the concentration of constituents in the inflow water, $\mathrm{mg} / \mathrm{ml}$, and $\mathrm{R}$ is the flow rate, $\mathrm{min} / \mathrm{ml}$. 
For experimental purposes, pilot-scale CWTSs were used due to their sufficiently small size to facilitate control and manipulation of macrofeatures (e.g. water depth, hydraulic retention time, vegetation, and hydrosoil; Dorman et al. 2009; Rodgers and Castle, 2008). For this experiment, wetland cells were constructed in $150 \mathrm{~L}$ polypropylene barrels that were $34.5 \mathrm{~cm}$ in diameter and $75.5 \mathrm{~cm}$ deep. The wetland cells were connected by polyvinyl chloride (PVC) pipe fittings and arranged to permit flow of water to each cell in series by gravity. The PVC pipe fittings were positioned at $30 \mathrm{~cm}$ from the bottom of the polypropylene barrel approximately $15 \mathrm{~cm}$ above the sediment with a standpipe to adjust the water depth internally to allow for changes in the wetland cells during the experiment. Six replicated series were constructed for this pilot-scale experiment with four wetland cells in each series.

For this study, experimental water depths were determined from the film theory of mass transfer and NOSR equations. Values calculated from the film theory of mass transfer equation generated a linear model $(y=-0.0001 x+0.0022)$ containing reaeration rates and the corresponding depths from 0 to $60 \mathrm{~cm}$. Five values related to specific water depths and reaeration rates were used in the NOSR equation $(y=-0.0001 x-0.0491)$ to predict the supply of oxygen to the sediment. Theoretically, these five water depths would produce oxidizing (i.e. $15,23,33 \mathrm{~cm}$ ) or reducing (i.e. 46 and $56 \mathrm{~cm}$ ) sediment conditions in the wetland cells. Five water depths were grouped according to predicted sediment redox conditions [1)15 and $23 \mathrm{~cm}$ water depths, 2) $33 \mathrm{~cm}$ water depth, and 3) 46 and $56 \mathrm{~cm}$ water depths]. For experimental purposes, two wetland series were used for each water depth group. The standpipes were positioned according to a specific water depth for each series. For the initial experiment, standpipes were positioned for the 15, 33, and $46 \mathrm{~cm}$ water depths in the wetland series. For the subsequent experiment, standpipes were changed in length to produce the 23, 33, and $56 \mathrm{~cm}$ water depths. During this study, the $33 \mathrm{~cm}$ water depth series were not altered to establish commonality between the two experiments. At the initiation of the experiment and between changes in water depths, pilot-scale systems were allowed to acclimate. An acclimation period is the time needed to obtain stability of a system as reflected by sediment redox potential, $\mathrm{pH}$, and dissolved oxygen concentration (OECD, 2002), and the period of acclimation in this case was approximately two to three weeks. Sediment for all wetland cells was from Eighteen Mile Creek (South Carolina) and composed of $\sim 85 \%$ sand, $\sim 15 \%$ silt, and clay-size particles. Vegetation in the first two wetland cells of each series was giant bulrush (S. californicus). Narrowleaf cattail $(T$. angustifolia) was planted in the final two wetland cells of each series. Initial density of these plants was 8-10 plants $/ \mathrm{m}^{2}$ in each wetland cell. Approximately three grams of Osmocote ${ }^{\circledR}$ Outdoor \& Indoor Smart-Release ${ }^{\circ}$ Plant Food (19-6-12 formula) were added to each wetland cell every two weeks for the duration of the experiment.

For complex mixtures such as FOPWs, a hybrid CWTS may be necessary to achieve the required performance to meet specific water use criteria. For this experiment, the hybrid system was comprised of an energy-driven component (i.e. oil/water separator) followed by a specifically designed pilot-scale CWTS. For FOPWs containing sufficient oil to merit separation, an oil/water separator may promote additional performance and opportunity to recover cost of operation and maintenance. For this pilot-scale free water surface CWTS, 
simulated FOPW was mixed with a $3 / 4$ horsepower sump pump in the simulated FOPW reservoir. From the reservoir to the oil/water separator, FMI ${ }^{\circledR}$ piston pumps were calibrated to maintain a $6 \mathrm{~h}$ HRT to allow for sufficient flow to achieve the targeted HRT in the pilot-scale systems. After exiting the oil/water separator, simulated FOPW flowed to each series using FMI ${ }^{\circledR}$ pumps. The equation relating water depth to HRT (Eq. 8) was used to calculate flow rates for each series depending on the corresponding water depth. FMI $^{\circledR}$ pumps were calibrated for the pilot-scale systems to the corresponding flow rates to maintain a $36 \mathrm{~h} \mathrm{HRT}$ for each water depth in the wetland cells.

Aqueous samples were collected from three locations: simulated FOPW reservoir, oil/water separator outflow, and each wetland cell outflow in the pilot-scale CWTSs. Cd, $\mathrm{Cu}, \mathrm{Ni}, \mathrm{Zn}$, and oil were measured to determine if treatment performance was achieved for surface water discharge and irrigation in the pilot-scale CWTSs (Table 2). Aqueous Cd, $\mathrm{Cu}, \mathrm{Ni}$, and $\mathrm{Zn}$ samples $(50 \mathrm{~mL})$ were collected bi-weekly in Nalgene bottles and prepared for analysis within 48 hours of collection (APHA, 2005). Aqueous samples for oil analysis were collected from each wetland cell monthly using 1 liter glass jars using the APHA (2005) method (5520 A) and a sheen net at the water's surface according to the ASTM (2011) method (D4489-95) and prepared for analysis within four hours of collection (APHA, 2005). Cd, Cu, Ni, Zn, and oil samples were stored at $4{ }^{\circ} \mathrm{C}$ until analysis at Clemson University (APHA, 2005). Soluble $\mathrm{Cd}, \mathrm{Cu}, \mathrm{Ni}$, and $\mathrm{Zn}$ concentrations were prepared by filtering aqueous samples through a $0.45-\mu \mathrm{m}$ filter, acidifying to $\mathrm{pH}<2$ with trace metal grade nitric acid (Thermo Fisher Scientific, Inc.), and analyzing using Inductively Coupled Plasma-Optical Emission Spectroscopy (ICP-OES; Perkin Elmer; USEPA, 1994; APHA, 2005). According to USEPA (1993), soluble metal concentrations may be measured to determine bioavailable constituents in contaminated waters (Allen and Hansen, 1996). Oil samples were prepared by liquid-liquid extraction (Korda et al. 1997). The sample flowed $\left(10 \mathrm{ml} / \mathrm{min}\right.$ ) into a silica column (Supelclean ${ }^{\mathrm{TM}} \mathrm{ENVI}^{\mathrm{TM}}-18 \mathrm{SPE}$ cartridge; Li and Lee, 2001). Flow rate was controlled by adjusting the vacuum suction pressure. Oil compounds were eluted from the column using $10 \mathrm{ml}$ chloroform [moderately insoluble (MI)], then $10 \mathrm{ml} n$-hexane [water insoluble (WI)], and $10 \mathrm{ml}$ methanol [water soluble (WS); Siron et al. 1987]. WI, MI, and WS eluates were dried under a nitrogen current to a volume of $2 \mathrm{ml}$ to inject in vials for analysis (Siron et al. 1987). Dried WI, MI, and WS eluates were analyzed initially for spectral identification of compounds for the use of calibration standards to estimate concentrations of those compounds (MDEP, 2009). WI and MI compounds (1,2-Benzofluorenone and 1-Methylcyclopentanol, respectively) were analyzed using gas chromatography (GC; Varian 8510) with a flame ionization detector using an ZB-1 capillary column (50m length, i.d. 0.3mm; Siron et al. 1987; Korda et al. 1997). Temperature was programmed for the $\mathrm{GC}$ from 170 to $290^{\circ} \mathrm{C}$ at $3^{\circ} \mathrm{C} \mathrm{min}{ }^{-1}$ with a retention time of 45 minutes and the carrier gas was helium (Siron et al. 1987). The WS compound (Diethylhydroxypentanol) was analyzed using high pressure liquid chromatography (HPLC; Dionex UltiMate 3000; Siron et al. 1987) with an Acclaim Polar Advantage II column $(4.6 \times 250 \mathrm{~mm}, 5 \mu \mathrm{m}, 120 \AA)$. Temperature was programmed on the HPLC from $30-45^{\circ} \mathrm{C}$ at $5^{\circ} \mathrm{C} \mathrm{min}^{-1}$ with a retention time of 30 minutes (Siron et al. 1987). The following gradient elution was applied—solvent A: acetonitrile; solvent B: water at time $0: \mathrm{A}=40 \%, \mathrm{~B}=60 \%$; time $5 \mathrm{~min}: \mathrm{A}=40 \%, \mathrm{~B}=60 \%$; time $40 \mathrm{~min}: \mathrm{A}=100 \%, \mathrm{~B}=$ 0\% (Moret et al. 1997). 
Water $\mathrm{pH}$, temperature, conductivity, dissolved oxygen, alkalinity, and hardness were measured bi-weekly from each wetland cell (APHA, 2005). Temperature and water $\mathrm{pH}$ were measured with an Orion model GDT-11 field probe, and dissolved oxygen and conductivity were measured using a YSI meter model 85 from the inflow and each wetland cell (APHA, 2005). Sediment redox potential was measured bi-weekly in each wetland cell. Sediment redox potential was measured with a platinum electrode and an $\mathrm{Ag} / \mathrm{AgCl}$ reference system (accuracy: $\pm 10 \mathrm{mV}$ ) at $2.5 \mathrm{~cm}$ depth in the middle of each wetland cell (Faulkner et al. 1989).

Sediment redox potential and organic matter content are parameters that may be influenced by mass loading rates and plant detritus decomposition which can potentially affect treatment performance of the system. Wetland cells were compared within and between each water depth group to discern differences in the sediment redox potential and organic matter content. Sediment redox potential was measured as described previously, and organic matter content in the sediment was measured at the initiation and termination of this experiment from each wetland cell. Organic matter content was measured from the top $2.5 \mathrm{~cm}$ of sediment using loss-on-ignition method (USEPA, 2002d).

Data collected for actual and simulated FOPW for $\mathrm{Cd}, \mathrm{Cu}, \mathrm{Ni}, \mathrm{Zn}$, oil, and salts were analyzed for normal distribution. Significant differences in the $\mathrm{Cd}, \mathrm{Cu}, \mathrm{Ni}, \mathrm{Zn}$, oil, and salt concentrations for the actual and simulated FOPWs were determined by ANOVA and Tukey's procedure $(\alpha=0.05)$. Treatment performance data were analyzed for normal distribution. Significant differences in mean outflow concentrations of the five water depth wetland cells of the pilot-scale systems were determined by ANOVA and Tukey's procedure $(\alpha=0.05)$. Sediment redox potential and organic matter content for the different water depths and wetland cells containing $S$. californicus and $T$. angustifolia were analyzed for normal distribution. Significant differences in sediment redox potential and organic matter content for the different water depths and wetland cells containing S. californicus and $T$. angustifolia were determined by ANOVA and Tukey's procedure $(\alpha=0.05)$. Statistical analyses were conducted using Statistical Analytical System (SAS) 9.2 (SAS Institute, 2002).

Statistical comparisons using ANOVA and Tukey's procedure were conducted on the different water depths for treatment performance. There is a significant difference $(\alpha=0.05)$ between the water depths for concentrations of $\mathrm{Cd}, \mathrm{Cu}, \mathrm{Ni}, \mathrm{Zn}$, and oil fractions. For the 15,23 , and $33 \mathrm{~cm}$ water depths, the $\mathrm{Cd}, \mathrm{Ni}$, and oil fraction concentrations were not significantly different. For Zn concentrations, the 15 and $23 \mathrm{~cm}$ and the 23 and $33 \mathrm{~cm}$ water depths were not significantly different from each other. For $\mathrm{Cu}$ concentrations in the wetlands, the $15 \mathrm{~cm}$ water depth was significantly different from the other depths, while the 23 and $33 \mathrm{~cm}$ water depths and the 33, 46, and $56 \mathrm{~cm}$ water depths were not significantly different from each other. $\mathrm{Cd}, \mathrm{Cu}, \mathrm{Ni}, \mathrm{Zn}$, and oil concentrations were not significantly different $(\alpha=0.05)$ for the 46 and $56 \mathrm{~cm}$ water depths. The following $\mathrm{Cd}, \mathrm{Cu}$, $\mathrm{Ni}, \mathrm{Zn}$, and oil outflow concentrations are presented as two different categories: wetland cells with oxidizing conditions in the sediment (i.e. 15, 23, and $33 \mathrm{~cm}$ ) and wetland cells with reducing conditions in the sediment (i.e. 46 and $56 \mathrm{~cm}$ ). 
Average inflow concentrations ( \pm standard deviation) of the simulated FOPW were 3.99 $( \pm 1.23) \mathrm{mg} \mathrm{Cd} / \mathrm{L}, 3.31( \pm 2.49) \mathrm{mg} \mathrm{Cu} / \mathrm{L}, 3.74( \pm 0.82) \mathrm{mg} \mathrm{Ni} / \mathrm{L}$, and $3.77( \pm 1.04) \mathrm{mg} \mathrm{Zn} / \mathrm{L}$. The average inflow oil concentration and after the oil/water separator were $43.73( \pm 1.59)$ $\mathrm{mg} / \mathrm{L}$ and 14.15 ( \pm 1.09$) \mathrm{mg} / \mathrm{L}$ (Table 59).

The average soluble concentrations exiting the $46 \mathrm{~cm}$ water depth constructed wetland were $0.16( \pm 0.15) \mathrm{mg} \mathrm{Cd} / \mathrm{L}, 0.04( \pm 0.03) \mathrm{mg} \mathrm{Cu} / \mathrm{L}, 0.07( \pm 0.03) \mathrm{mg} \mathrm{Ni} / \mathrm{L}$, and $0.15( \pm 0.11)$ $\mathrm{mg} \mathrm{Zn/L} \mathrm{(Figures} \mathrm{35-38;} \mathrm{sediment} \mathrm{redox} \mathrm{potential} \mathrm{(SRP):}-65$ to $-168.9 \mathrm{mV}$ ). At a water depth of $56 \mathrm{~cm}$, the average soluble concentrations exiting the constructed wetland were $0.19( \pm 0.27) \mathrm{mg} \mathrm{Cd} / \mathrm{L}, 0.06( \pm 0.08) \mathrm{mg} \mathrm{Cu} / \mathrm{L}, 0.09( \pm 0.06) \mathrm{mg} \mathrm{Ni} / \mathrm{L}$, and $0.18( \pm 0.22) \mathrm{mg}$ $\mathrm{Zn} / \mathrm{L}$ (SRP: -132.8 to $-212 \mathrm{mV}$; Figures 36-39). The average $\mathrm{Cd}, \mathrm{Cu}$, and Ni concentrations had a slight increase as the water depth increased from 46 to $56 \mathrm{~cm}$. Significant differences $(\alpha=0.05)$ were observed for the $\mathrm{Cd}, \mathrm{Cu}, \mathrm{Ni}$, and $\mathrm{Zn}$ concentrations in the inflow water and 46 and $56 \mathrm{~cm}$ water depth wetland cell outflows. Removal of $\mathrm{Cd}, \mathrm{Cu}, \mathrm{Ni}$, and $\mathrm{Zn}$ in the wetland cells may be attributed to the reducing environment conducive for metal precipitation by sulfides through dissimilatory sulfate reduction.

The average oil concentrations from the initial to the final cell for the $46 \mathrm{~cm}$ water depth wetland cells were $13.89( \pm 0.59) \mathrm{mg} / \mathrm{L}, 13.73( \pm 0.61) \mathrm{mg} / \mathrm{L}, 13.59( \pm 0.52) \mathrm{mg} / \mathrm{L}$, and $13.48( \pm 0.49) \mathrm{mg} / \mathrm{L}$. The average oil concentrations after each $56 \mathrm{~cm}$ water depth wetland cell were $13.67( \pm 0.85) \mathrm{mg} / \mathrm{L}, 13.49( \pm 0.76) \mathrm{mg} / \mathrm{L}, 13.39( \pm 0.71) \mathrm{mg} / \mathrm{L}$, and $13.35( \pm 0.69)$ $\mathrm{mg} / \mathrm{L}$. We expected more removal in the oxidizing wetland cells (i.e. 15, 23, and $33 \mathrm{~cm}$ water depth wetland cells) than in the reducing wetland cells (i.e. 46 and $56 \mathrm{~cm}$ water depth wetland cells) which had a slower degradation rate (Figure 40; Table 59).

The average soluble concentrations exiting the $15 \mathrm{~cm}$ water depth constructed wetland were $3.38( \pm 0.99) \mathrm{mg} \mathrm{Cd} / \mathrm{L}, 1.78( \pm 0.81) \mathrm{mg} \mathrm{Cu} / \mathrm{L}, 3.54( \pm 0.47) \mathrm{mg} \mathrm{Ni} / \mathrm{L}$, and $3.55( \pm 0.57)$ $\mathrm{mg} \mathrm{Zn/L} \mathrm{(SRP:} 162$ to $250.1 \mathrm{mV}$ ). The average soluble concentrations exiting the $23 \mathrm{~cm}$ water depth constructed wetland were $3.94( \pm 1.25) \mathrm{mg} \mathrm{Cd} / \mathrm{L}, 2.23( \pm 2.23) \mathrm{mg} \mathrm{Cu} / \mathrm{L}, 3.02$ $( \pm 1.31) \mathrm{mg} \mathrm{Ni} / \mathrm{L}$, and $2.74( \pm 0.84) \mathrm{mg} \mathrm{Zn} / \mathrm{L}$ (SRP: 115.8 to $249.5 \mathrm{mV})$. The average soluble concentrations exiting the $33 \mathrm{~cm}$ water depth constructed wetland were 3.01 $( \pm 0.97) \mathrm{mg} \mathrm{Cd} / \mathrm{L}, 1.29( \pm 1.78) \mathrm{mg} \mathrm{Cu} / \mathrm{L}, 2.74( \pm 0.83) \mathrm{mg} \mathrm{Ni} / \mathrm{L}$, and $2.44( \pm 0.73) \mathrm{mg} \mathrm{Zn} / \mathrm{L}$ (SRP: 15.3 to $138.4 \mathrm{mV}$ ). We observed decreases in the divalent metal concentrations as the depth increased from 15 to $33 \mathrm{~cm}$ (Figures 36-39; Table 59). Divalent metal outflow concentrations were greater in wetland cells having 15 and $23 \mathrm{~cm}$ water depths than in cells having 46 and $56 \mathrm{~cm}$ water depths. The 46 and $56 \mathrm{~cm}$ water depth wetland cells provided reducing conditions conducive for dissimilatory sulfate reduction. Precipitation of divalent metals by acid-volatile sulfide binding requires reducing conditions in the sediment in order to provide the appropriate environment for dissimilatory sulfate reducing microbial assemblages.

The average oil concentrations from the initial to the final cell for the $15 \mathrm{~cm}$ water depth wetland cells were $10.26( \pm 0.16) \mathrm{mg} / \mathrm{L}, 7.52( \pm 0.35) \mathrm{mg} / \mathrm{L}, 3.12( \pm 0.66) \mathrm{mg} / \mathrm{L}$, and 0.96 $( \pm 0.07) \mathrm{mg} / \mathrm{L}(\mathrm{SRP}: 162$ to $250.1 \mathrm{mV})$. The average oil concentrations for the $23 \mathrm{~cm}$ water depth wetland cells were $10.29( \pm 0.51) \mathrm{mg} / \mathrm{L}, 6.92( \pm 1.65) \mathrm{mg} / \mathrm{L}, 3.07( \pm 0.47) \mathrm{mg} / \mathrm{L}$, and 
$1.08( \pm 0.14) \mathrm{mg} / \mathrm{L}$. The average oil concentrations for the $33 \mathrm{~cm}$ water depth wetland cells were $10.92( \pm 0.59) \mathrm{mg} / \mathrm{L}, 8.33( \pm 0.27) \mathrm{mg} / \mathrm{L}, 5.31( \pm 0.30) \mathrm{mg} / \mathrm{L}$, and $2.52( \pm 0.09) \mathrm{mg} / \mathrm{L}$. We observed a greater decrease in oil concentrations in the 15 and $23 \mathrm{~cm}$ water depths wetland cells than in the $33 \mathrm{~cm}$ water depth wetland cell. This may be attributed to a lower redox potential in the sediment (i.e. less oxygen to the sediment) of the $33 \mathrm{~cm}$ than the 15 and $23 \mathrm{~cm}$ water depth wetland cells. As water depth increases, the concentration of oil removed decreases (Figure 40; Table 59). We observed lower oil concentrations in the 15 and $23 \mathrm{~cm}$ water depths than in the 46 and $56 \mathrm{~cm}$ water depths wetland cells (Figure 40). It is generally concluded that oil degrades more quickly under aerobic conditions than under anaerobic conditions (Zobell, 1964; Hughes and McKenzie, 1975; Ward and Brock, 1978; Delaune et al. 1980).

Water depth influences sediment redox in a wetland, which may alter treatment performance of a system. The 15 and $23 \mathrm{~cm}$ water depth wetland cells were not efficient for removing $\mathrm{Cd}, \mathrm{Cu}, \mathrm{Ni}$, and $\mathrm{Zn}$ concentrations from the simulated FOPW. However, the 15 and $23 \mathrm{~cm}$ water depths wetland cells were more effective for removing oil components than the 46 and $56 \mathrm{~cm}$ water depth wetland cells since aerobic degradation occurs at a faster rate than anaerobic degradation. The 46 and $56 \mathrm{~cm}$ water depth wetland cells were more effective at removing $\mathrm{Cd}, \mathrm{Cu}, \mathrm{Ni}$, and $\mathrm{Zn}$ from simulated FOPW than the 15, 23, and $33 \mathrm{~cm}$ water depth wetland cells. The $33 \mathrm{~cm}$ water depth redox potential (i.e. 15.3 to 138.4 $\mathrm{mV}$ ) was inefficient for providing conditions conducive for divalent metal precipitation and degradation of oil and, as a result, was ineffective at removing these constituents from simulated FOPW. Differences in the water depth used for Phase II of this project was 40.6 $\mathrm{cm}$ with a HRT of $24 \mathrm{~h}$, while the water depth experiment wetland cells contained water depths from 15 to $56 \mathrm{~cm}$ in depth and a HRT of $36 \mathrm{~h}$. In comparison, the wetland cells having a $40.6 \mathrm{~cm}$ water depth removed more divalent metals than the 46 and $56 \mathrm{~cm}$ wetland cells. This may be attributed to the wetland cell size and maturity of the system.

Removal rate coefficients were calculated using Equation 9 for the 5 water depths investigated. For the 15,23 , and $33 \mathrm{~cm}$ water depths, oxidizing conditions $(+15.3$ to $+250.1 \mathrm{mV})$ were established along with greater removal rates for oil (0.01-0.056 day-1) compared with the 46 and $56 \mathrm{~cm}$ water depths ( 0.001 day-1). Reducing environments (65 to $-212 \mathrm{mV}$ ) were generated in 46 and $56 \mathrm{~cm}$ water depths and supported greater removal rates for metals (0.01-0.185 day-1) than the $15,23,33$, cm water depths (no change-0.077 day-1), which may be attributed to precipitation by sulfides from dissimilatory sulfate reduction. 


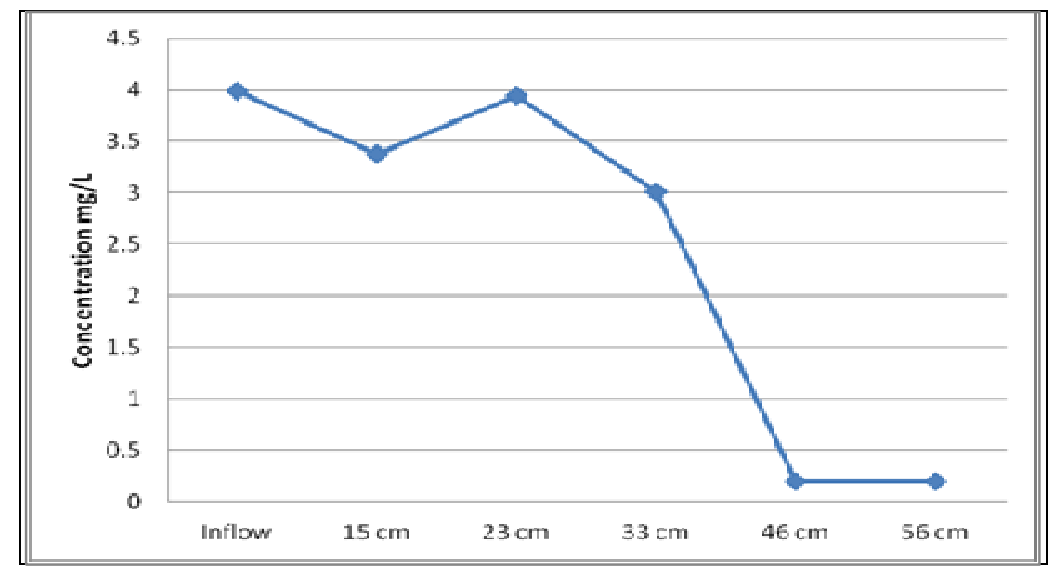

Figure 36. Average cadmium concentrations in the inflow and outflow for each water depth in the constructed wetland.

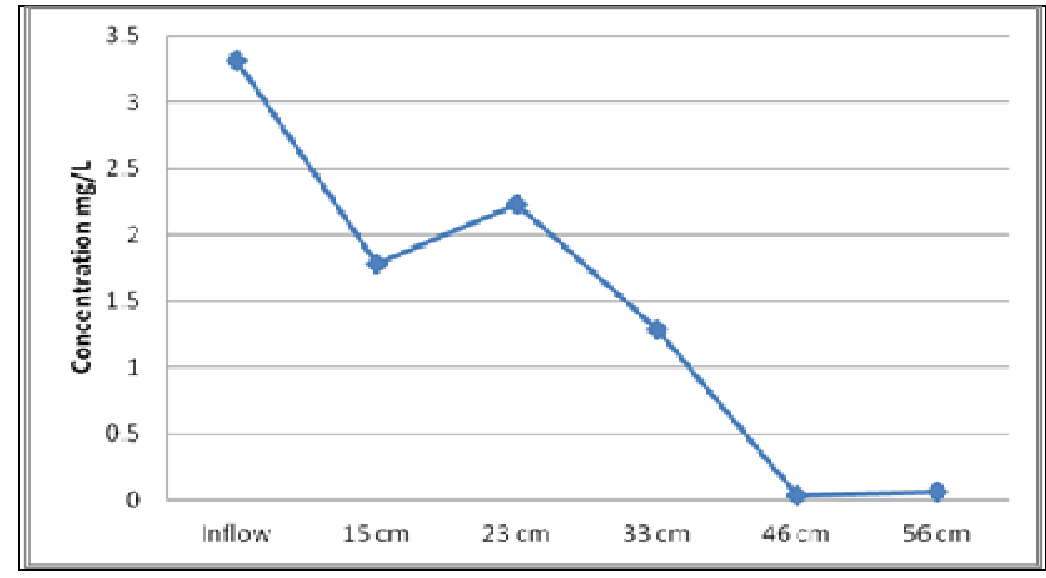

Figure 37. Average copper concentrations in the inflow and outflow for each water depth in the constructed wetland. 


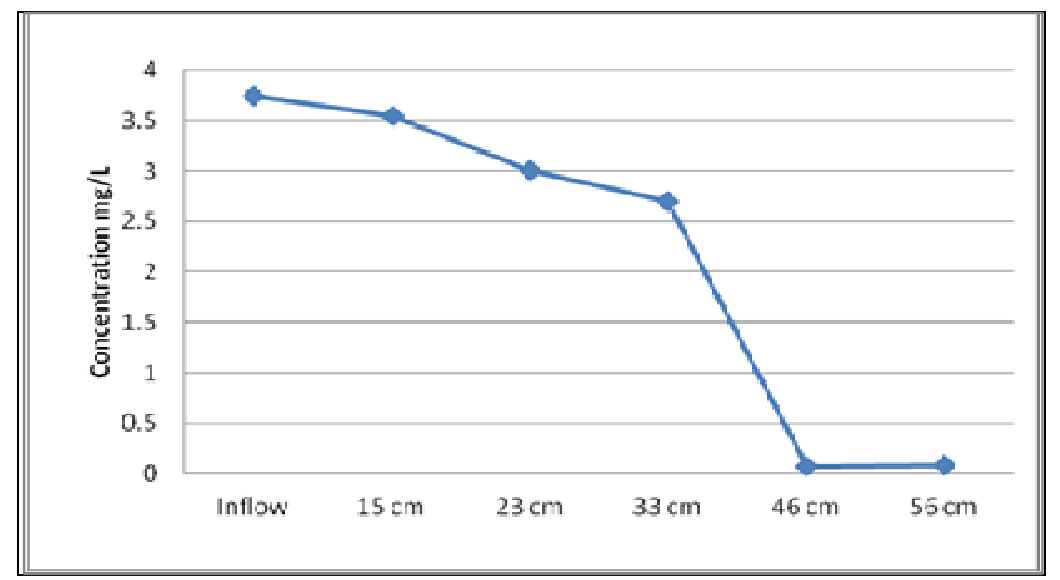

Figure 38. Average nickel concentrations in the inflow and outflow for each water depth in the constructed wetland.

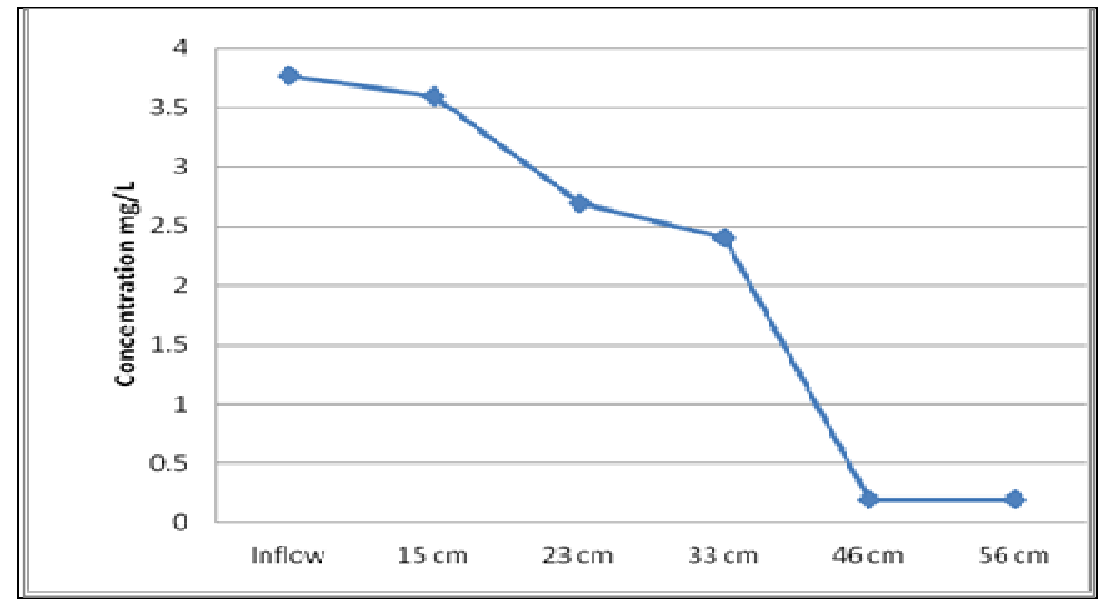

Figure 39. Average zinc concentrations in the inflow and outflow for each water depth in the constructed wetland. 


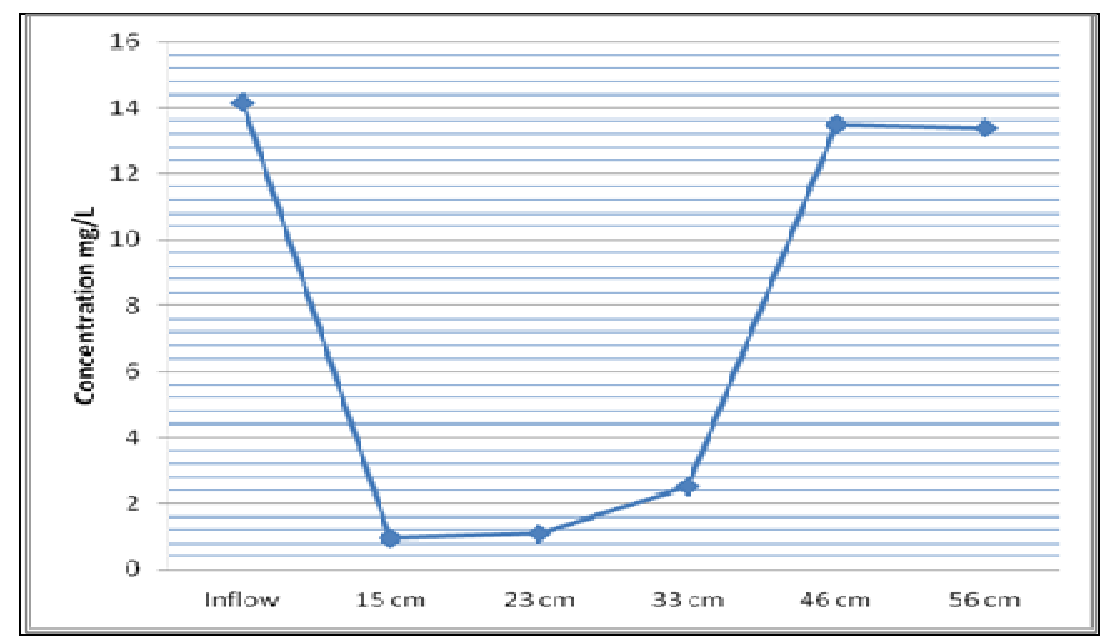

Figure 40. Average oil concentrations in the inflow and outflow for each water depth in the constructed wetland.

Table 59. Average constituent concentrations in the simulated FOPW at various water depths.

\begin{tabular}{lrllll}
\hline & \multicolumn{1}{c}{ Oil } & $\mathrm{Cd}$ & $\mathrm{Cu}$ & $\mathrm{Ni}$ & $\mathrm{Zn}$ \\
\hline Inflow & 14.15 & 3.99 & 3.31 & 3.74 & 3.77 \\
$15 \mathrm{~cm}$ & 0.96 & 3.38 & 1.78 & 3.54 & 3.55 \\
$23 \mathrm{~cm}$ & 1.08 & 3.94 & 2.23 & 3.02 & 2.74 \\
$33 \mathrm{~cm}$ & 2.52 & 3.01 & 1.29 & 2.74 & 2.44 \\
$46 \mathrm{~cm}$ & 13.48 & 0.16 & 0.04 & 0.07 & 0.15 \\
$56 \mathrm{~cm}$ & 13.35 & 0.19 & 0.06 & 0.09 & 0.18 \\
\hline
\end{tabular}

\section{Seasonal Variation}

Among aquatic systems, constructed wetlands display the greatest seasonal variability of physical and chemical characteristics (e.g. macrophytes, sedimentation rates, flow volume, type and concentration of constituents) in response to terrestrial and climatic events (Kalff, 2002; Rai, 2008). Seasonal variability may have direct or indirect effects on biological processes that may be temperature dependent (Abdollahi and Nedwell, 1979; Halda-Alija and Johnston, 1999). Biological processes targeted for constituent removal in pilot-scale CWTSs include biodegradation of oil by heterotrophic aerobic microbial assemblages and precipitation of $\mathrm{Cd}, \mathrm{Cu}, \mathrm{Ni}$, and $\mathrm{Zn}$ by sulfides through dissimilatory sulfate reduction.

Responses of microbial populations to changes in temperature are crucial knowledge for successful design and implementation of a full-scale CWTS. Investigations were conducted concerning the effect of seasonal variability on performance of a hybrid pilotscale CWTS for the removal of $\mathrm{Cd}, \mathrm{Cu}, \mathrm{Ni}, \mathrm{Zn}$, and oil. For this experiment, the hybrid system was comprised of an energy-driven component (i.e. oil/water separator) followed 
by a specifically designed pilot-scale CWTS (Figure 41). For FOPWs containing sufficient oil to merit separation, an oil/water separator may promote additional performance and opportunity to recover cost of operation and maintenance. The objective of this research was to measure the seasonal performance of a hybrid pilot-scale constructed wetland system for treatment of $\mathrm{Cd}, \mathrm{Cu}, \mathrm{Ni}, \mathrm{Zn}$, and oil in simulated FOPW.

Simulated FOPW was formulated based on constituent concentrations in FOPWs reported in peer-reviewed literature (Veil et al., 2004; Thordsen et al., 2007; Alley et al., 2011) and government documents (USEPA, 2000; USGS, 2002) as well as analyses of PW samples provided from oilfield sites in the continental United States. For simulated FOPW, an ionic balance was calculated (i.e. $\Sigma$ cations $(\mathrm{meq} / \mathrm{L})+\Sigma$ anions $(\mathrm{meq} / \mathrm{L})$ from analyte concentrations in actual FOPW to formulate this water with appropriate salts to accurately achieve ionic composition and strength as well as salinity and hardness. The oil component for the simulated FOPW formulation was conventional light oil (Rotella ${ }^{\circledR} \mathrm{T}$ 15W40), a cost-effective and reproducible alternative to using an actual crude oil. General water characteristics (i.e. $\mathrm{pH}$, dissolved oxygen, conductivity, alkalinity, and hardness) were also measured to compare simulated FOPW and actual FOPW. Simulated FOPW was composed of $\mathrm{Cd}, \mathrm{Cu}, \mathrm{Ni}, \mathrm{Zn}$, oil, and salts well-mixed with $3780 \mathrm{~L}$ of municipal water in a reservoir (USEPA, 2000; USGS, 2002; Veil et al., 2004; Thordsen et al., 2007; Alley et al., 2011). This formulation of FOPW resulted in the following concentrations of targeted constituents required for treatment: $6 \mathrm{mg} \mathrm{Cd}^{+2} / \mathrm{L}, 4 \mathrm{mg} \mathrm{Cu}^{+2} / \mathrm{L}, 6 \mathrm{mg} \mathrm{Ni}^{+2} / \mathrm{L}, 6 \mathrm{mg} \mathrm{Zn}^{+2} / \mathrm{L}$, and $100 \mathrm{mg}$ Rotella® T 15W40/L.

For this pilot-scale free water surface CWTS, simulated FOPW was mixed with a $3 / 4$ horsepower sump pump in the simulated FOPW reservoir. From the reservoir to the oil/water separator, FMI® piston pumps were calibrated to maintain a $6 \mathrm{~h}$ HRT to allow for sufficient flow to achieve the targeted HRT in the pilot-scale systems. The nominal HRT of the pilot-scale CWTS was 24 hours per cell or 96 hours for the series.

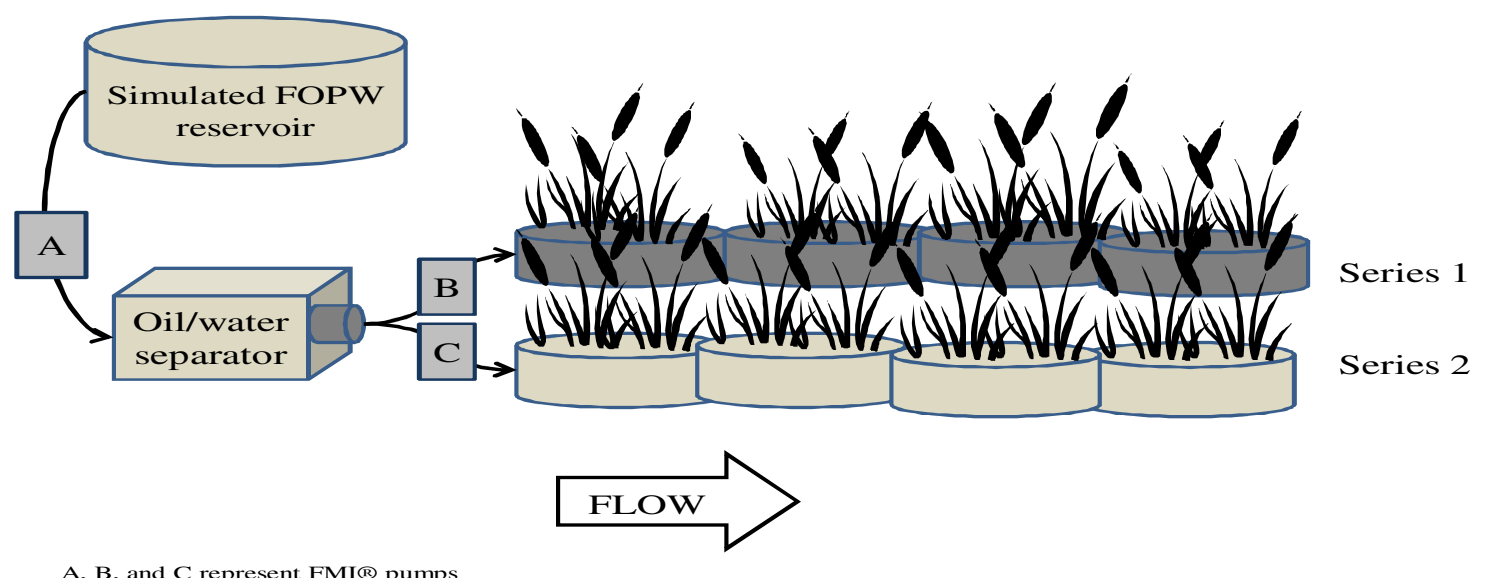

Figure 41. Schematic of the hybrid pilot-scale constructed wetland treatment systems 
Simulated FOPW samples were collected and analyzed at Clemson University from May 2010 through May 2011. Aqueous samples were collected from three locations: simulated FOPW reservoir, oil/water separator outflow, and each wetland cell outflow in the pilotscale CWTSs. Cd, $\mathrm{Cu}, \mathrm{Ni}, \mathrm{Zn}$, and oil were targeted constituents measured to determine whether treatment performance was achieved for surface water discharge and irrigation in the pilot-scale CWTSs (Table 60). Aqueous $\mathrm{Cd}, \mathrm{Cu}, \mathrm{Ni}$, and $\mathrm{Zn}$ samples $(50 \mathrm{~mL}$ ) were collected bi-weekly in Nalgene bottles and prepared for analysis within 48 hours of collection (APHA, 2005). Aqueous samples for oil analysis were collected from each wetland cell monthly using 1 liter glass jars using the APHA (2005) method (5520 A) and a sheen net at the water's surface according to the ASTM (2011) method (D4489-95) and prepared for analysis within four hours of collection (APHA, 2005). These samples were stored at $4^{\circ} \mathrm{C}$ until analysis at Clemson University (APHA, 2005). Acid-soluble $\mathrm{Cd}, \mathrm{Cu}$, $\mathrm{Ni}$, and $\mathrm{Zn}$ concentrations were prepared by acidifying to $\mathrm{pH}<2$ with trace metal grade nitric acid, filtering aqueous samples through a $0.45-\mu \mathrm{m}$ filter (Thermo Fisher Scientific, Inc.), and analyzing using Inductively Coupled Plasma-Optical Emission Spectroscopy (ICP-OES; Perkin Elmer; USEPA, 1994b; APHA, 2005). Oil samples were prepared by liquid-liquid extraction (Korda et al. 1997). The sample flowed $(10 \mathrm{ml} / \mathrm{min}$ ) into a silica column (Supelclean ${ }^{\mathrm{TM}} \mathrm{ENVI}^{\mathrm{TM}}$ - 18 SPE cartridge; Li and Lee, 2001). The flow rate was controlled by adjusting the vacuum suction pressure. Oil compounds were eluted from the column using $10 \mathrm{ml}$ chloroform [moderately insoluble (MI)], then $10 \mathrm{ml} n$-hexane [water insoluble (WI)], and $10 \mathrm{ml}$ methanol [water soluble (WS); Siron et al. 1987]. The WI, MI, and WS eluates were dried under a nitrogen current to a volume of $2 \mathrm{ml}$ to inject in vials for analysis (Siron et al. 1987). Dried WI, MI, and WS eluates were analyzed initially for spectral identification of compounds for the use of calibration standards to estimate concentrations of those compounds (MDEP, 2009). WI and MI compounds (1,2Benzofluorenone and 1-Methylcyclopentanol, respectively) were analyzed using gas chromatography (GC; Varian 8510) with a flame ionization detector using an ZB-1 capillary column (50m length, i.d. 0.3mm; Siron et al. 1987; Korda et al. 1997).

Temperature was programmed for the $\mathrm{GC}$ from 170 to $290^{\circ} \mathrm{C}$ at $3^{\circ} \mathrm{C} \mathrm{min}^{-1}$ with a retention time of 45 minutes and the carrier gas was helium (Siron et al. 1987). The WS compound (Diethylhydroxypentanol) was analyzed using high pressure liquid chromatography (HPLC; Dionex UltiMate 3000; Siron et al. 1987) with an Acclaim Polar Advantage II column $(4.6 \times 250 \mathrm{~mm}, 5 \mu \mathrm{m}, 120 \AA$ ). Temperature was programmed on the HPLC from 30$45^{\circ} \mathrm{C}$ at $5^{\circ} \mathrm{C} \mathrm{min}^{-1}$ with a retention time of 30 minutes (Siron et al. 1987). The following gradient elution was applied—solvent A: acetonitrile; solvent B: water at time $0: \mathrm{A}=40 \%$, $\mathrm{B}=60 \%$; time $5 \mathrm{~min}: \mathrm{A}=40 \%, \mathrm{~B}=60 \%$; time $40 \mathrm{~min}: \mathrm{A}=100 \%, \mathrm{~B}=0 \%$ (Moret et al. 1997).

Water $\mathrm{pH}$, temperature, conductivity, dissolved oxygen, alkalinity, and hardness were measured and recorded bi-weekly from each wetland cell (APHA, 2005). Temperature and water $\mathrm{pH}$ were measured with an Orion model GDT-11 field probe, and dissolved oxygen and conductivity was measured using a YSI meter model 85 from the inflow and each wetland cell (APHA, 2005). Sediment redox potential was measured and recorded biweekly in each wetland cell. Sediment redox potential was measured with a platinum electrode and an $\mathrm{Ag} / \mathrm{AgCl}$ reference system (accuracy: $\pm 10 \mathrm{mV}$ ) at a $2.5 \mathrm{~cm}$ depth in the 
near middle of each wetland cell (Faulkner et al. 1989). Performance data were analyzed for normal distribution. Significant differences in mean outflow concentrations between the two wetland cell series were determined by ANOVA and Tukey's procedure $(\alpha=$ $0.05)$.

There were no observed differences $(\alpha=0.05)$ in the performance of the two wetland series for oil, $\mathrm{Cd}, \mathrm{Cu}, \mathrm{Ni}$, and $\mathrm{Zn}$. Average ( \pm s.d.) oil concentration exiting Series 1 was 1.32 $( \pm 0.28) \mathrm{mg}$ oil compounds/L. Average ( \pm s.d.) oil concentration exiting Series 2 was 1.3 $( \pm 0.29) \mathrm{mg}$ oil compounds/L. Both wetland series remediated approximately $82.0-91.4 \%$ of the oil compounds measured in the simulated FOPW samples. Approximately 98.399.9\% of the divalent metal concentrations in the simulated FOPW were renovated by the pilot-scale system. Average ( \pm s.d.) $\mathrm{Cd}, \mathrm{Cu}, \mathrm{Ni}$, and $\mathrm{Zn}$ concentrations exiting Series 1 were 0.003 ( \pm 0.002$) \mathrm{mg} \mathrm{Cd} / \mathrm{L}, 0.004( \pm 0.002) \mathrm{mg} \mathrm{Cu} / \mathrm{L}, 0.063( \pm 0.028) \mathrm{mg} \mathrm{Ni} / \mathrm{L}$, and 0.051 $( \pm 0.041) \mathrm{mg} \mathrm{Zn} / \mathrm{L}$ (Figure 42). For Series $1, \mathrm{Cu}$ concentrations measured in July, September, and October were non-detect and were recorded for calculation purposes as the detection limit (0.003). Average ( \pm s.d.) $\mathrm{Cd}, \mathrm{Cu}, \mathrm{Ni}$, and $\mathrm{Zn}$ concentrations exiting Series 2 were $0.003( \pm 0.002) \mathrm{mg} \mathrm{Cd} / \mathrm{L}, 0.004( \pm 0.002) \mathrm{mg} \mathrm{Cu} / \mathrm{L}, 0.053( \pm 0.034) \mathrm{mg} \mathrm{Ni} / \mathrm{L}$, and $0.052( \pm 0.038) \mathrm{mg} \mathrm{Zn} / \mathrm{L}$ (Figure 43). Differences in removal efficiencies for $\mathrm{Cd}, \mathrm{Cu}, \mathrm{Ni}$, and $\mathrm{Zn}$ may be attributed to the Ksp values for sulfide complexation (i.e. $\mathrm{Ksp}: \mathrm{Cu} 10^{-37}, \mathrm{Cu}$ $10^{-32}$, and $\sim \mathrm{Ni}$ and $\mathrm{Zn} 10^{-25}$ ) or preferential uptake by S. californicus or T. angustifolia (Kamal et al. 2004). The temperature ranged from 2.7 to $30.2^{\circ} \mathrm{C}$, and average temperatures for each month are illustrated in Figure 44. No observed significant differences $(\alpha=0.05)$ in performance of either series were affected by changes in temperature in the pilot-scale system. Performance data for wetland series 1 and 2 were compared to FAO irrigation guidelines and USEPA water quality criteria (Table 60). During the experiment, $\mathrm{Cd}, \mathrm{Cu}$, $\mathrm{Ni}, \mathrm{Zn}$, and oil outflow concentrations met FAO irrigation guidelines, however, $\mathrm{Cd}$ and oil compound concentrations did not continuously met the USEPA water quality criteria. Although chemical-specific data met the irrigation and surface water discharge criteria, toxicity data were needed to determine if any synergist or additive effects occurred between the constituents in the simulated FOPW. 


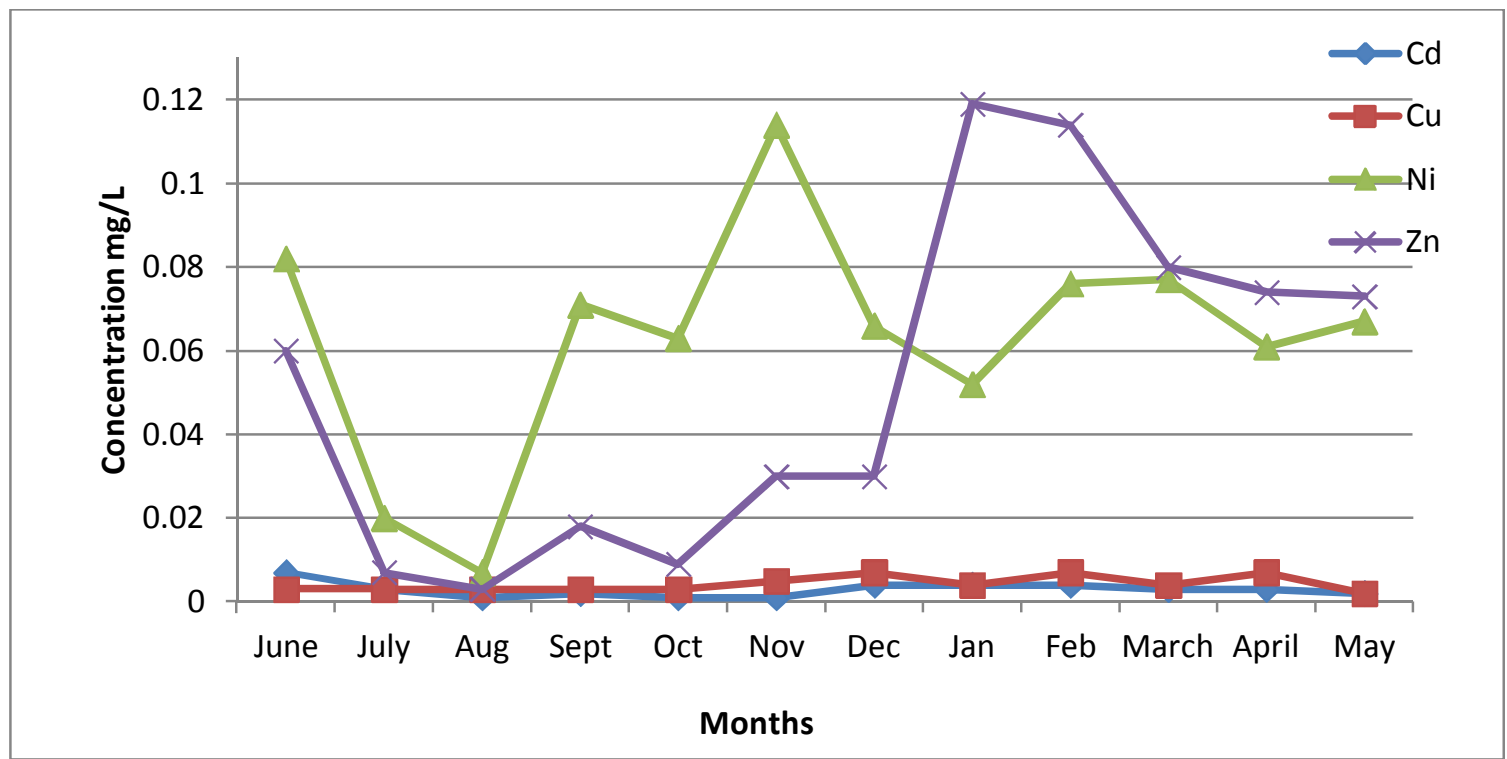

Figure 42. Average outflow concentrations of $\mathrm{Cd}, \mathrm{Cu}, \mathrm{Ni}$, and $\mathrm{Zn}$ for Series 1 wetland

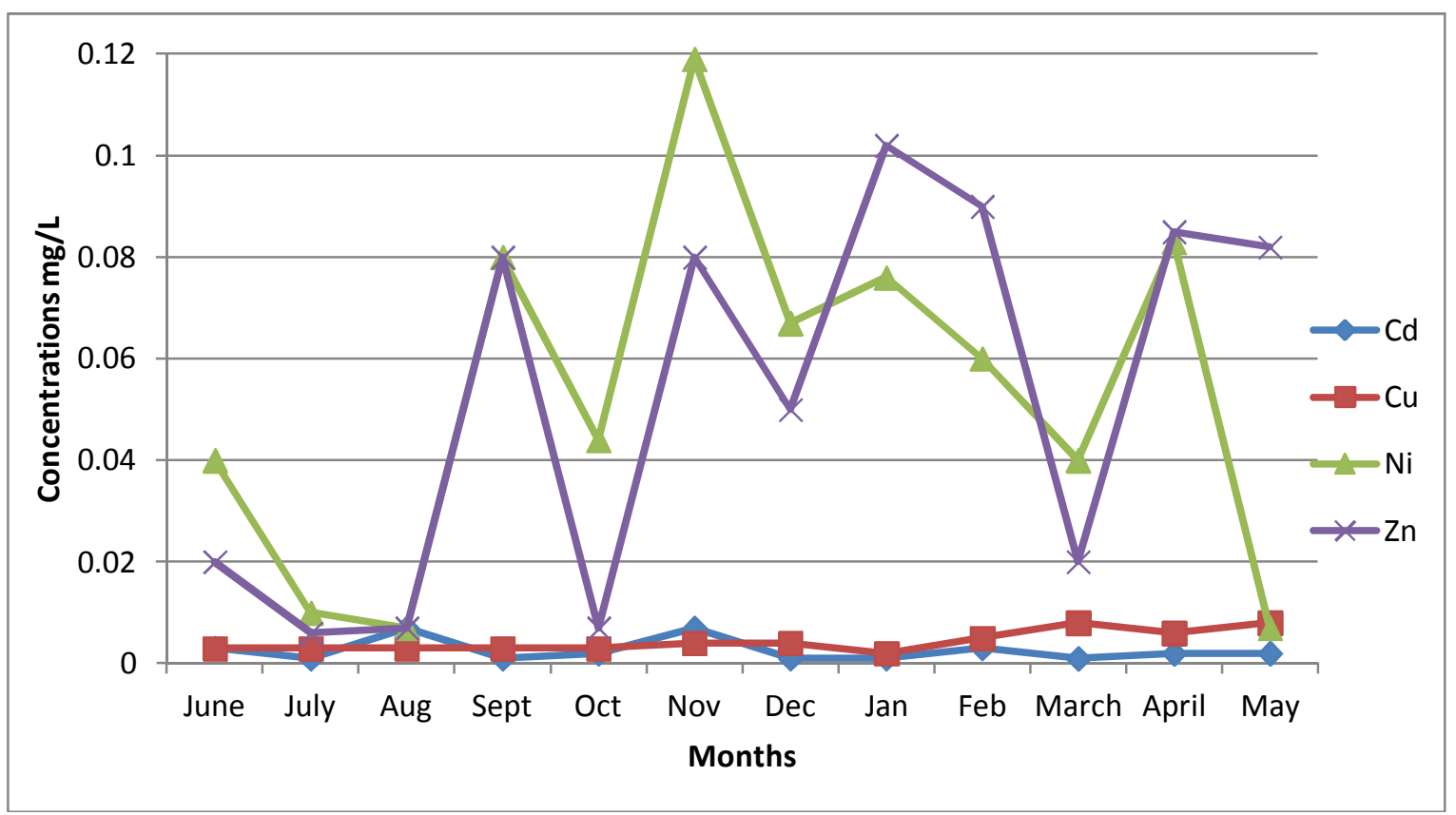

Figure 43. Average outflow concentrations of $\mathrm{Cd}, \mathrm{Cu}, \mathrm{Ni}$, and $\mathrm{Zn}$ for Series 2 wetland 


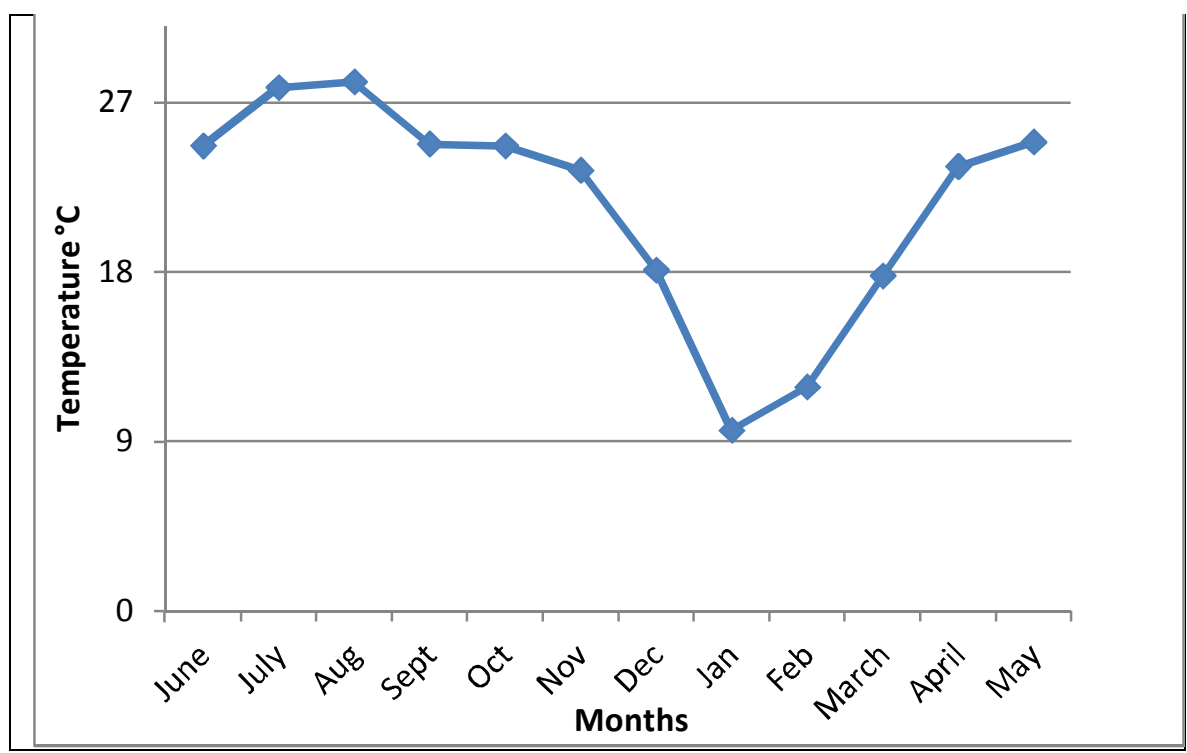

Figure 44. Average temperatures for each month during the pilot-scale CWTS experiment

Table 60. Comparisons between FAO Irrigation Guidelines and USEPA Water Quality Criteria and Outflow Concentrations (mg/L) from Constructed Wetland Series 1 and 2.

\begin{tabular}{cccccc}
\hline & Cadmium & Copper & Nickel & Zinc & Oil \\
\cline { 2 - 6 } Inflow & 3.99 & 3.31 & 3.74 & 3.77 & 42.78 \\
Series 1 & 0.003 & 0.004 & 0.063 & 0.051 & 1.32 \\
Series 2 & 0.003 & 0.004 & 0.053 & 0.052 & 1.3 \\
Irrigation Guidelines & 0.01 & 0.2 & 0.2 & 2 & 35 \\
Surface Discharge & 0.002 & 0.009 & 0.47 & 0.12 & 0.1 \\
\hline
\end{tabular}

Toxicity experiments using fish and invertebrate species and crop seedlings (i.e. corn, cabbage, and soybean) were conducted to discern if this simulated FOPW was suitable for use (i.e. surface water discharge and irrigation) after treatment.

Toxicity experiments are important for determining if a water may cause adverse effects to receiving system biota. Responses (i.e. mortality) of Ceriodaphnia dubia Richard and Pimephales promelas Rafinesque can be used to evaluate the toxicity of treated and untreated simulated FOPW. C. dubia and P. promelas are sentinel species used as indicators for synergistic, additive, and antagonistic effects of constituents that may occur in simulated FOPW. Untreated and treated simulated FOPWs were collected from series inflow, after the oil/water separator, and after each wetland cell. USEPA (1994b) method 600/4-91/002 for estimating toxicity of receiving waters was modified by changes in the composition of moderately hard water used in the method. C. dubia and P. promelas mortality was measured in 7-day static/renewal toxicity experiments $(n=20 P$. promelas per treatment and $\mathrm{n}=10$ C. dubia per treatment: USEPA 2002c). Statistical analysis of the responses of $C$. dubia and $P$. promelas was conducted using SAS 9.2 (SAS Institute 2002) to determine if differences existed between a laboratory control and untreated and treated 
simulated fresh PW. Differences were determined by performing Chi-Square Analysis $(\alpha=$ 0.05; SAS Institute 2002). The null hypothesis, untreated simulated fresh PW equivalent in toxicity to treated simulated fresh PW, was rejected by the Chi-square analysis ( $\mathrm{p}$ $<0.0001$ ). There is a significant difference between the responses (i.e. mortality) of $C$. $d u b i a$ and $P$. promelas to untreated and treated simulated fresh PW. All of the $C$. dubia and $P$. promelas died in the untreated simulated fresh PW within 24 hours. After 7 days, nine $C$. dubia and eight $P$. promelas survived in water treated by wetland series 1 , and eight $C$. $d u b i a$ and eight $P$. promelas survived in water treated by wetland series 2 .

One-way ANOVA and Post-hoc Tukey's procedure $(\alpha=0.05)$ were used to evaluate early seedling growth of corn (Zea maya), cabbage (Brassica oleracea), and soybean (Phaseolus vulgaris) seeds with control (municipal) water ( $\mathrm{n}=30$ for each seed group), untreated ( $\mathrm{n}=30$ for each seed group) and treated simulated FOPW ( $\mathrm{n}=30$ for each seedling group). For early seedling growth, differences in the root and shoot length between the seeds watered with treated and untreated simulated FOPW were significant $(\alpha=0.05)$. The control and treated seedlings were not significantly different $(\alpha=0.05)$ in root and shoot length. All of the seedlings in the control and treated groups were green and grew twice as fast as the untreated groups. For example, after Day 3 the average soybean height for the control and treated groups was approximately $5.8 \mathrm{~cm}$, while the average soy bean height for the untreated group was approximately $3.9 \mathrm{~cm}$. Only six out of twenty-five untreated cabbage seedlings grew, but were yellow and wilted. Because of their smaller size, the cabbage seeds may have been more sensitive to the constituents than the other seeds. Survival rate was greater for the corn and soybean seedlings than for the cabbage. All of the corn and soybean untreated seedlings grew, but the leaf tips turned yellow and eventually brown. The treated groups continued to grow during the experiment and appeared green and healthy. Green, healthy seedlings were grown from the treated simulated PW, but not from the untreated simulated FOPW. Rate of seedling growth was greater for the treated water than for the untreated water.

An experiment was conducted to discern possible differences in AVS concentrations in the hydrosoil with changes in seasonal temperature. Production of sulfides in the wetland can indicate the presence and activity of dissimilatory sulfate reducing microbial assemblages. Following the method of Leonard and others (1996), acid-volatile sulfides (AVS) were measured as an indirect indicator of dissimilatory sulfate reduction in the pilot-scale CWTSs. A statistical analysis using a 2-sample T-test $(\alpha=0.05)$ was done to determine if production of AVS was temperature-dependent. The analysis indicated no difference ( $\mathrm{p}$ value $=0.31$ ) between the AVS concentrations produced between the winter and summer months. The consistent removal of divalent metals through the winter and summer months may be attributed to the steady production of sulfides by dissimilatory sulfate reducing microbial assemblages or storage of sulfides in the sediment. 


\section{Nutrient Amendment}

The purpose of this research was to gain an understanding of Se-sequestering biogeochemical processes and evaluate the effects of a nutrient amendment on those biogeochemical processes, hydrosoil conditions (redox, $\mathrm{pH}$, and organic matter percentage), Se accumulation, and Se-reducing microbial colonies in the hydrosoil of CWTSs. The two pilot-scale CWTSs [nutrient amended (AquaSmart ${ }^{\mathrm{TM}}$ ) vs. unamended (control)] used in this study were designed and constructed to reduce aqueous $\mathrm{Se}$ concentrations in simulated produced water containing low molecular weight organics and metalloids. The specific objectives of this study were: (i) measure hydrosoil conditions, Se-reducing microbial colony forming units (CFUs), and Se accumulation vertically through the hydrosoil; (ii) investigate the potential for Se-sequestering biogeochemical processes (reduction, sorption/complexation) operating in the hydrosoil at various depths; and (iii) evaluate the effect of a nutrient amendment on hydrosoil conditions, microbial CFUs, Se accumulation, and potential for Se-sequestering biogeochemical processes in a pilot-scale CWTS cell designed to treat Se in PW.

This study utilized the first cell from two of the pilot-scale CWTSs (unamended and nutrient amended) designed and constructed in Phase II to treat low molecular weight organics and metalloids in simulated produced water. In the nutrient amended cell and unamended cell, detritus zone grab samples ( $7 \mathrm{~cm}$ intervals) and sediment zone samples ( $3 \mathrm{~cm}$ intervals) were collected (Figure 45$)$. The nutrient amended cell's vertical hydrosoil profile consisted of three 7-cm thick detritus zone samples $(0-21 \mathrm{~cm})$ and five $3-\mathrm{cm}$ thick sediment zone samples $(21-36 \mathrm{~cm})$ while the unamended cell's profile contained two $7-\mathrm{cm}$ thick detritus zone samples $(0-14 \mathrm{~cm})$ and five 3 -cm thick sediment zone samples (14-29 $\mathrm{cm})$. To accomplish the first objective, redox potential was measured in-situ in the hydrosoil before sampling, and $\mathrm{pH}$ and organic matter percentage were measured in each sample. Se concentrations were determined for each sample using a microwave digestion procedure (CEM procedures: Microwave Sample Preparation Note, App. Note: OS-14) followed by analysis using an ICP-MS (EPA 200.8). Se-reducing CFUs were measured from pore water samples collected at each sampling interval of the hydrosoil in both cells. The second objective was accomplished by comparing the hydrosoil conditions and Se concentrations within the nutrient amended cell and unamended cell to a literature review of the hydrosoil conditions in which Se-sequestering biogeochemical processes operate (Table 61). Additionally, vertical CFU counts in each cell were used to provide insight into the Se-sequestering biogeochemical processes that may be occurring vertically through the hydrosoil. The third objective was accomplished by comparing hydrosoil conditions, vertical CFU count and vertical Se concentration of the nutrient amended cell with the hydrosoil conditions, vertical CFU count and vertical Se concentration of the unamended cell.

The detritus zone in the nutrient amended pilot-scale system was $7 \mathrm{~cm}$ thicker than that of the unamended system. The hydrosoil of each cell was near or within the targeted redox potential range $(-150$ to $+50 \mathrm{mV}$ ) for dissimilatory Se reduction (Table 62 and Figure 46). The $\mathrm{pH}$ was circum-neutral ranging from 6.2-6.8 in the nutrient amended cell and 6.2-6.5 in the unamended cell (Table 62 and Figure 47). The percent of organic matter decreased 
with depth in the hydrosoil in the first cells of both pilot-scale CWTSs (88\% to $1 \%$ in the nutrient amended cell and 79\% to $1 \%$ in the unamended cell) (Figure 48). In each cell, the percentage of organic matter in the detrital zone was greater than in the sediment zone (Table 62). Se concentrations in each cell decreased with depth (Figure 49). Concentrations of Se were greater in the detrital zone (76-830 ppm in the nutrient-amended cell and 138$569 \mathrm{ppm}$ in the unamended cell) than in the sediment zone $(0.9-212 \mathrm{ppm}$ in the amended cell and 0.5-7 ppm in the unamended cell) (Table 62). Se-reducing microbes were present throughout the hydrosoil in both cells (Figure 50; Table 63).

Measured hydrosoil conditions were compared to hydrosoil conditions under which Se sequestering biogeochemical processes operate (Table 61). In the pilot-scale CWTS cells studied, the detritus zone and intervals of the sediment zone possessed favorable conditions for dissimilatory Se reduction and sorption/complexation (Table 61 and 62). Additionally, based on the high concentrations of Se measured in the detritus zone in the nutrient amended and unamended cells, Se sequestering biogeochemical processes are operating in the detritus zone. The percent of organic matter and Se concentrations in both cells decreased with depth and showed a similar trend suggesting that Se concentration may be influenced by organic matter. The presence of Se-reducing microbes in the hydrosoil of both cells indicates that dissimilatory Se-reduction can occur throughout the hydrosoil, but has the potential to operate at a greater rate in the hydrosoil where the number of CFUs is the greatest.

Redox potential in the hydrosoil of both cells ranged from $-150 \mathrm{mV}$ to $+50 \mathrm{mV}$, but differed vertically between the nutrient amended cell and unamended cell (Table 62; Figure 46). There was little difference in hydrosoil $\mathrm{pH}$ between the nutrient-amended cell and the unamended cell. Organic matter percent was greater in the nutrient amended cell than in the unamended cell (example: $79 \%$ at $7 \mathrm{~cm}$ in the unamended vs. $88 \%$ at $7 \mathrm{~cm}$ in the nutrient amended) (Table 62; Figure 48). Comparison of Se concentrations between the cells showed that the detritus zone of the nutrient amended cell contained higher concentrations of selenium than the detritus zone of the unamended cell (830 ppm vs. 569 ppm). Se concentration and organic matter percent in both cells decreased downward through the detritus zone, which contained higher Se concentrations and percent organic matter than the sediment zone (Figure 48 and 49; Table 62). Se-reducing CFUs were greater in hydrosoil of the nutrient amended cell than in hydrosoil of the unamended cell $(22,000 \mathrm{CFUs} / \mathrm{mL}$ pore water vs. $15,000 \mathrm{CFUs} / \mathrm{mL}$ pore water) (Figure 50; Table 63).

The greater concentrations of $\mathrm{Se}$ in the detritus zone compared to the sediment zone show the importance of the detritus zone for Se-sequestering biogeochemical processes and $\mathrm{Se}$ accumulation. Furthermore, the higher Se concentrations in the detritus zone of the nutrient amended cell as compared to the unamended cell may be attributed to the higher percent organic matter in the detritus zone of the nutrient amendment cell compared to the detritus zone of the unamended cell. The nutrient amendment (AquaSmart ${ }^{\mathrm{TM}}$ ) used in this study contained organic carbon, fermented yeast, and additional nutrients. In addition to providing a carbon source to promote Se-reducing microbial activity, nutrients contained in the AquaSmart ${ }^{\mathrm{TM}}$ amendment may have promoted T. latifolia growth within the nutrient amended cell and therefore increased the amount of $T$. latifolia litter falling onto the 
hydrosoil creating a thicker detritus zone. Selenium concentration, percent organic matter, and Se-reducing CFUs were greater in the nutrient amended cell, indicating a positive influence of the nutrient amendment on biogeochemical processes of Se accumulation and sequestration.

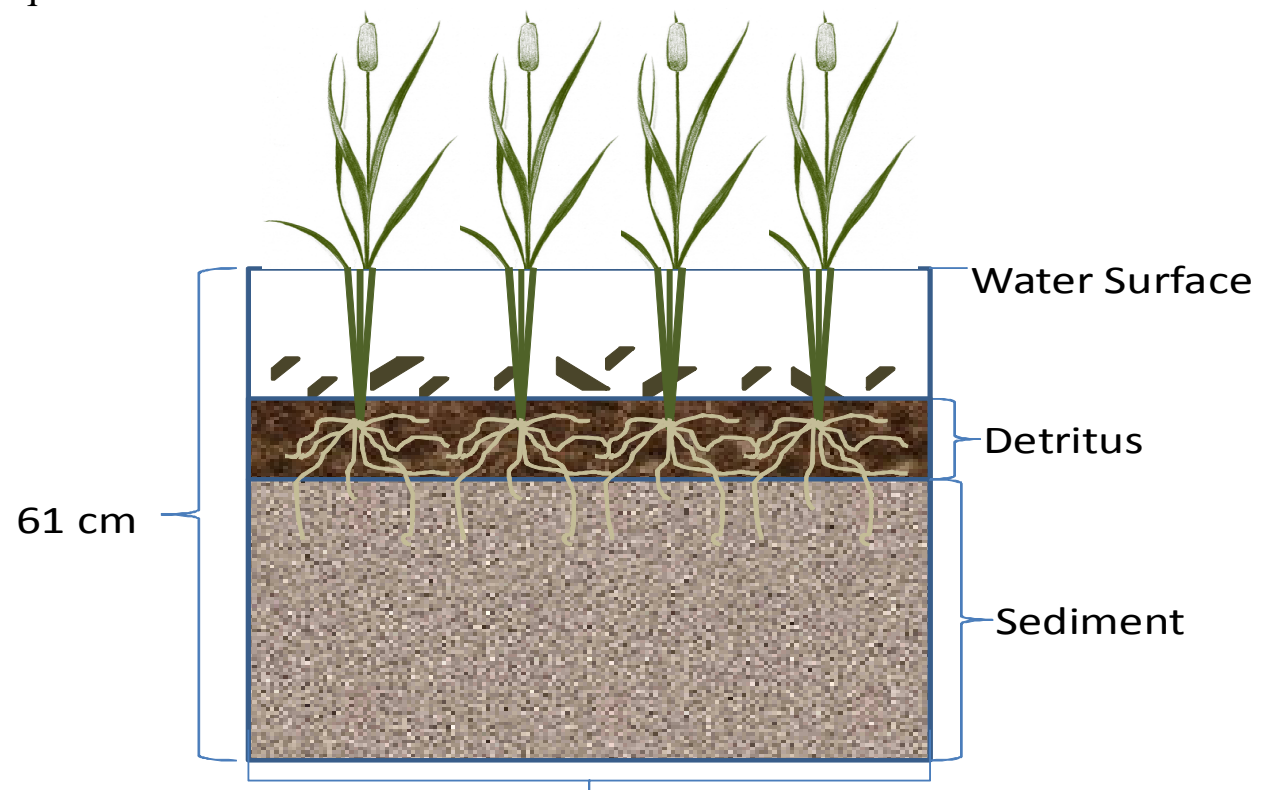

$124 \mathrm{~cm}$

Figure 45. Cross-section of a pilot-scale CWTS cell showing hydrosoil layers. Based on pilot-scale CWTS cell construction, observation, and deconstruction.
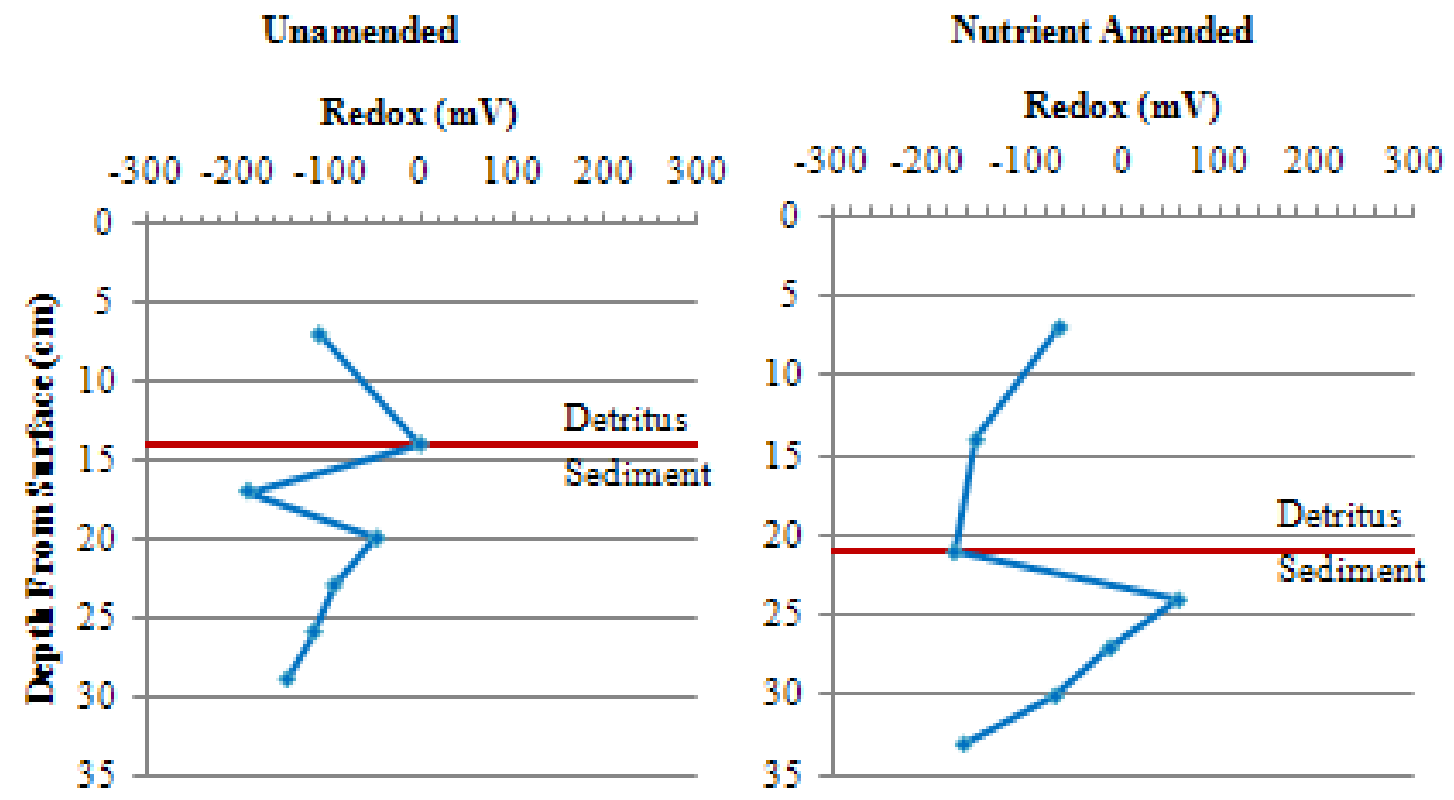

Figure 46. Redox with depth through the hydrosoil in the first cell (cell one) of each pilotscale CWTS. 
Unamended

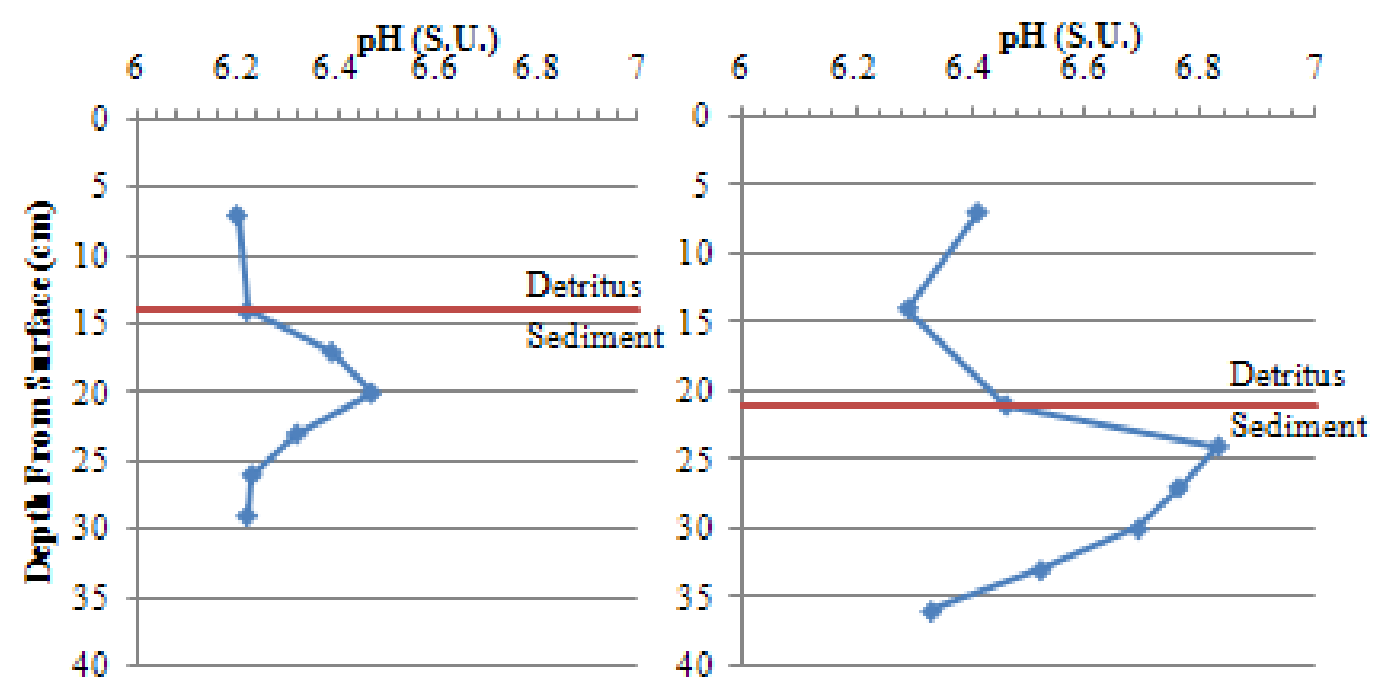

Figure 47. $\mathrm{pH}$ with depth through the hydrosoil in the first cell (cell one) of each pilotscale CWTS.
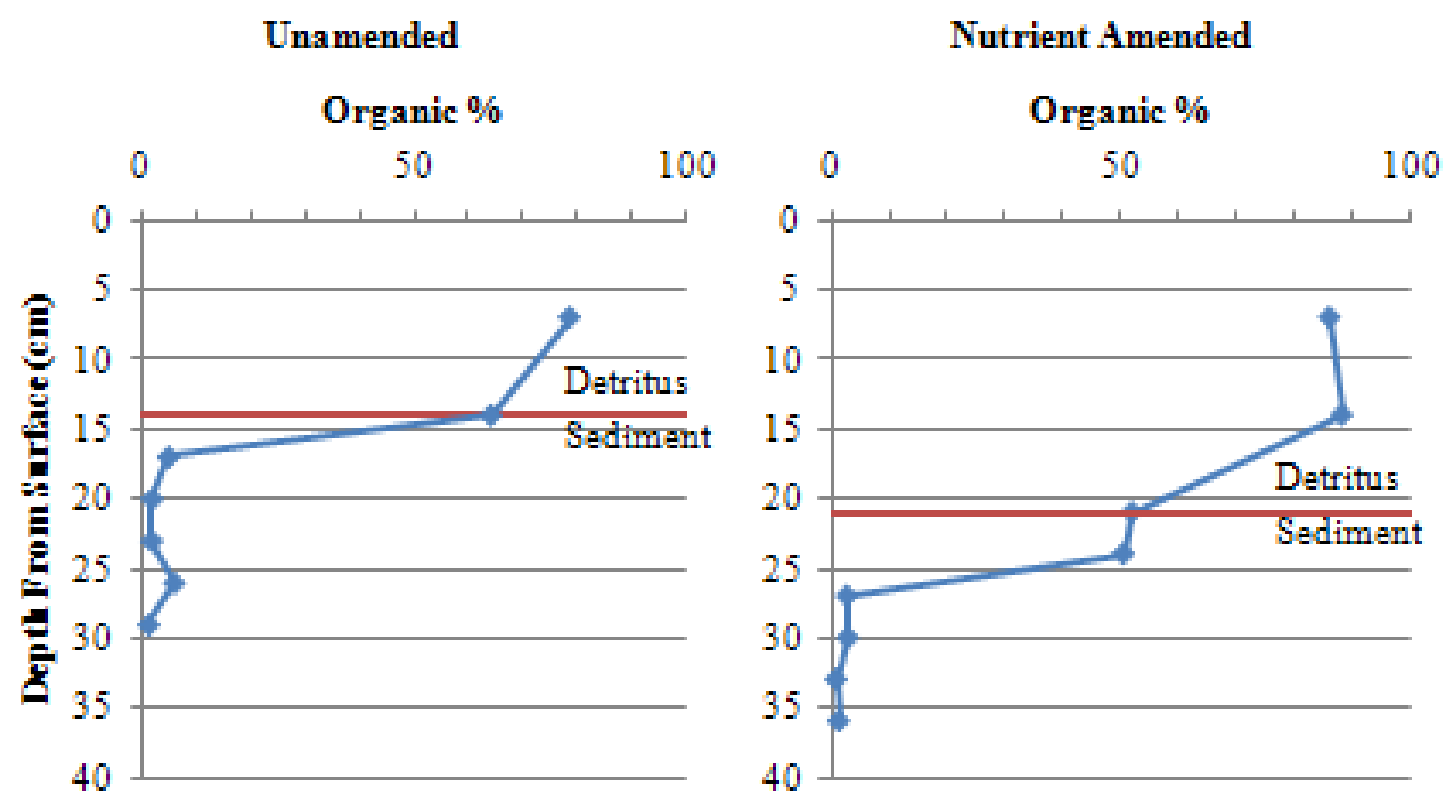

Figure 48. Organic matter percentage with depth through the hydrosoil in the first cell (cell one) of each pilot-scale CWTS. 

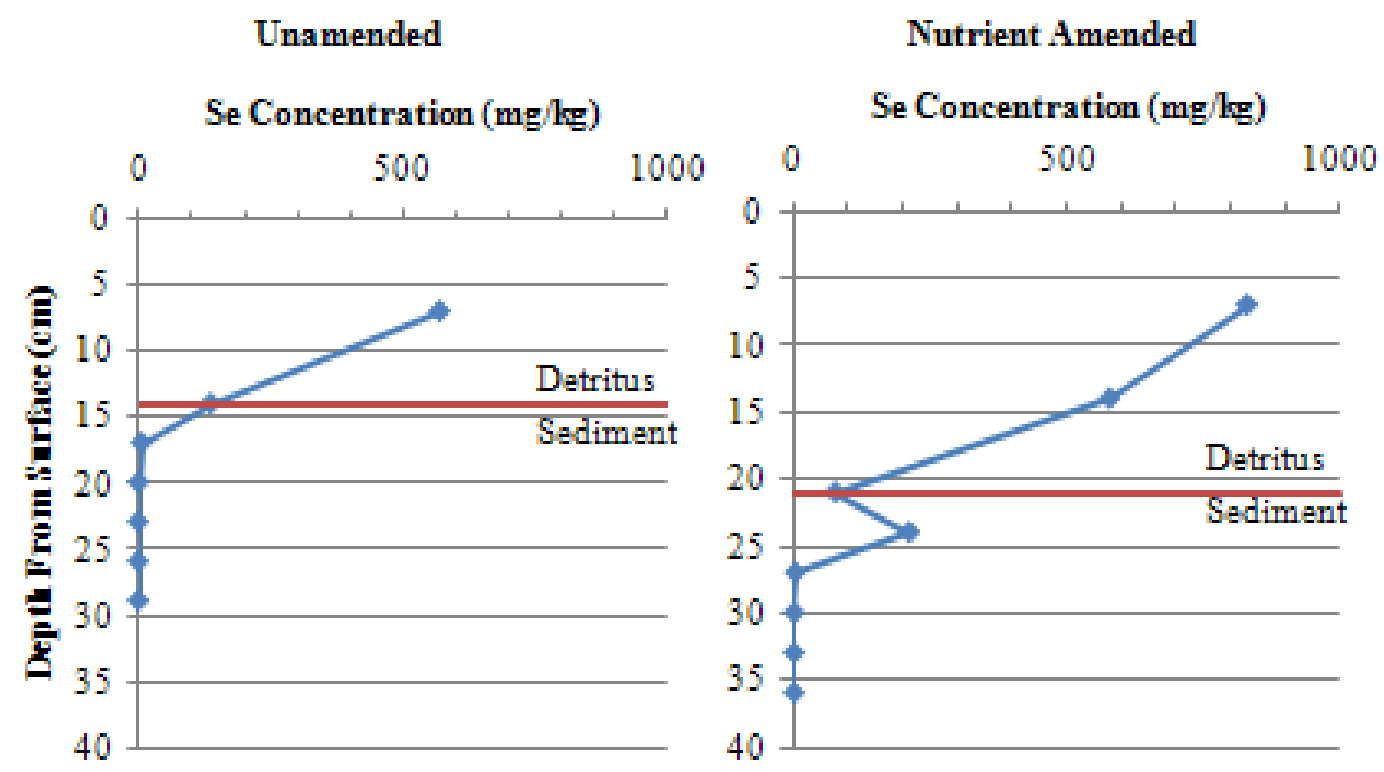

Figure 49. Se concentrations with depth through the hydrosoil in the first cell (cell one) of each pilot-scale CWTS.
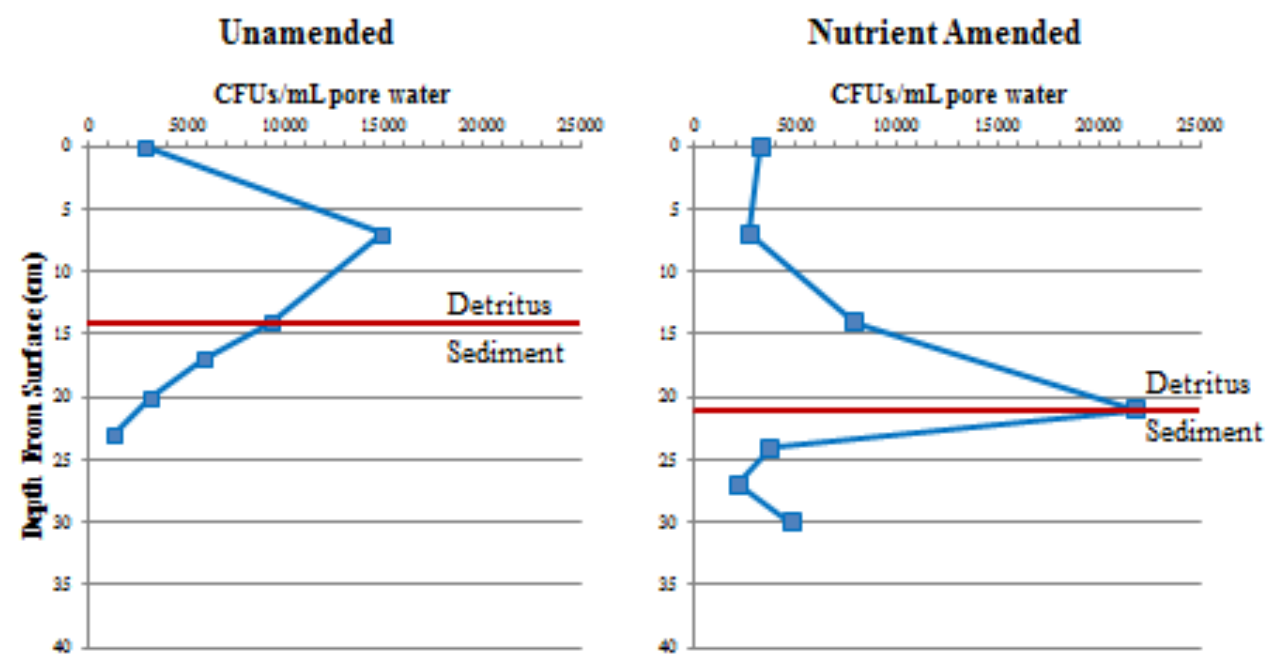

Figure 50. Se-reducing microbial colony forming units (CFUs) with depth through the hydrosoil in the first cell (cell one) of each pilot-scale CWTS. 
Table 61. Prevalent hydrosoil conditions for Se-sequestering biogeochemical processes within CWTSs.

\begin{tabular}{llll}
\hline Process & Organic Matter & $\mathrm{pH}$ & Redox Potential \\
\hline $\begin{array}{l}\text { Dissimilatory Se } \\
\text { Reduction }\end{array}$ & High $(>0.1 \%)$ & $\begin{array}{l}\text { Circum- Neutral } \\
(6.5-8)\end{array}$ & $\begin{array}{l}\text { Reducing } \\
(-150 \text { to }+50 \mathrm{mV})\end{array}$ \\
$\begin{array}{l}\text { Sorption/ Complexation } \\
\text { (organic \& inorganic) }\end{array}$ & High $(>6 \%)$ & $\begin{array}{l}\text { Acidic- Neutral } \\
(3-7)\end{array}$ & $\begin{array}{l}\text { Reducing-Oxidizing } \\
(-400 \text { to }+700 \mathrm{mV})\end{array}$ \\
\hline
\end{tabular}

(Trudinger and Swaine 1979, Shamberger 1983, de Souza et al. 1999, Selinus et al. 2005, Zhang et al. 2008, Rodgers and Castle 2008, Kadlec and Wallace 2009, Spacil 2010)

Table 62. Measured hydrosoil conditions and Se concentration with depth in nutrient amended and unamended CWTS cells.

\begin{tabular}{llllll}
\hline & Depth $(\mathrm{cm})$ & Redox $(\mathrm{mV})$ & $\mathrm{pH}(\mathrm{S} . \mathrm{U})$. & Organic Matter $(\%)$ & Total Se $(\mathrm{mg} / \mathrm{kg})$ \\
\hline Nutrient Amended Cell & & & & & \\
& $0-7$ & -65 & 6.41 & 86.08 & 830.19 \\
& $7-14$ & -151 & 6.29 & 88.28 & 578.67 \\
& $14-21$ & -173 & 6.46 & 52.04 & 76.33 \\
& $21-24$ & 57 & 6.83 & 50.57 & 211.91 \\
& $24-27$ & -14 & 6.76 & 2.89 & 3.28 \\
& $27-30$ & -70 & 6.69 & 3.08 & 1.17 \\
Unamended Cell & $30-33$ & -164 & 6.52 & 1.04 & 0.89 \\
& $33-36$ & -161 & 6.33 & 1.45 & 0.97 \\
& & & & & 569.07 \\
& $0-7$ & -111 & 6.20 & 78.72 & 137.61 \\
& $7-14$ & -2 & 6.22 & 64.26 & 6.77 \\
$14-17$ & -187 & 6.39 & 4.94 & 1.12 \\
& $17-20$ & -49 & 6.47 & 1.92 & 0.54 \\
$20-23$ & -95 & 6.32 & 1.89 & 0.65 \\
$23-26$ & -117 & 6.23 & 6.05 & 0.71 \\
\hline
\end{tabular}


Table 63. Measured Se-reducing microbial colony forming units (CFUs) with depth in nutrient amended and unamended CWTS cells.

\begin{tabular}{ccc}
\hline & Depth & CFUs/mL pore water \\
\hline Nutrient Amended Cell & & \\
& $0-7$ & 2700 \\
& $7-14$ & 8000 \\
& $14-21$ & 22000 \\
& $21-24$ & 3800 \\
& $24-27$ & 2200 \\
Unamended Cell & $27-30$ & 4800 \\
& $0-7$ & 15000 \\
& $7-14$ & 9300 \\
& $14-17$ & 5800 \\
& $17-20$ & 3200 \\
& $20-23$ & 1300 \\
\hline
\end{tabular}

\section{Oil-Water Separator}

PW containing increased mass loads of $\mathrm{O} \& \mathrm{G}$ provided the opportunity to evaluate the performance of a pilot-scale oil water separator (OWS) followed by a specifically designed constructed wetland treatment system (i.e., pilot-scale OWS and wetland cells, termed hybrid-constructed wetland system) for achieving desired treatment goals. The pilot-scale hybrid CWTS consisted of an OWS and four subsurface-flow cells, each with a 24-hr hydraulic retention time. The OWS was based on an American Petroleum Institute (API) design; which targeted the fraction of oil that can be separated by physical means. The OWS was designed to take advantage of the difference in specific gravity between oil and water. Dimensions of the OWS were $122 \mathrm{~cm}$ long by $61 \mathrm{~cm}$ wide by $33 \mathrm{~cm}$ deep. Treatment performance of the OWS and CWTS cells was assessed by measuring O \& G concentration in aqueous samples collected at the inflow of the OWS, outflow of the OWS, between each CWTS cell, and outflow from the CWTS.

Data from this experiment indicate that a hybrid CWTS produced a measureable decrease in outflow concentration of $\mathrm{O} \& \mathrm{G}(<1.4 \mathrm{mg} / \mathrm{L})$ compared to a CWTS not containing an OWS (9.9-14.6 mg/L), with both systems operating at an inflow concentration of 100 $\mathrm{mg} / \mathrm{L} \mathrm{O} \& \mathrm{G}$ (Figures 49 and 50). Both systems effectively decreased the concentration of $\mathrm{O} \& \mathrm{G}$ to below irrigation and livestock watering guidelines. Removal efficiencies (\%) and removal rate coefficients $(k)$ for O \& G were greater in the hybrid CWTS than in the CWTS only containing wetland cells (Table 64). This study has demonstrated that treatment performance in a CWTS is dependent on initial concentrations of $\mathrm{O} \& \mathrm{G}$; however, when increased mass loads of $\mathrm{O} \& \mathrm{G}$ are encountered, an OWS in a hybrid 
CWTS can contribute to effective treatment.

The effect of increased $\mathrm{O} \& \mathrm{G}$ mass loading by decreasing system HRT on treatment performance was investigated. Each series (SSF and FWS) was loaded with O \& G at 100 $\mathrm{mg} / \mathrm{L}$ targeted inflow concentration with an inflow rate providing a 4-day HRT (24-hr for each wetland cell), followed by loading at $100 \mathrm{mg} / \mathrm{L} \mathrm{O} \mathrm{\&} \mathrm{G} \mathrm{inflow} \mathrm{concentration} \mathrm{with} \mathrm{a} \mathrm{2-}$ day HRT (12-hr for each wetland cell). Nominal O \& G mass loading was $10 \mathrm{mg} / \mathrm{min}$ at $100 \mathrm{mg} / \mathrm{L}$ inflow concentration and 4-day HRT, and $20 \mathrm{mg} / \mathrm{min}$ at $100 \mathrm{mg} / \mathrm{L}$ inflow concentration and 2-day HRT.

For all cells in both series, redox potential was lower at $\sim 20 \mathrm{mg} / \mathrm{min}$ mass loading than at $\sim 10 \mathrm{mg} / \mathrm{min}$ mass loading (Table 65). For example, redox potential in the first cell of each series ranged from -130 (SSF) to $-147 \mathrm{mV}$ (FWS) for $20 \mathrm{mg} / \mathrm{min}$ loading and from 115 (SSF) to $98 \mathrm{mV}$ (FWS) for $10 \mathrm{mg} / \mathrm{min}$ loading. Oxidizing conditions (>-50 mV; Rodgers and Castle, 2008) were maintained in cell 4 in both series for all loadings of $O$ \& $\mathrm{G}$. For O \& G, in both the SSF and FWS series, removal extent was greater, removal rate coefficient was similar, and removal efficiency was less at $\sim 20 \mathrm{mg} / \mathrm{min}$ loading compared to $\sim 10 \mathrm{mg} / \mathrm{min}$ loading (Table 66). Outflow concentrations of all four metals increased after mass loading was increased from $\sim 10$ to $20 \mathrm{mg} / \mathrm{min}$, which is attributed to shorter HRT during $20 \mathrm{mg} / \mathrm{min}$ loading (Table 67). Rate coefficients increased for nickel (1.04 $\left.\mathrm{d}^{-1}\right)$ and zinc $\left(1.27 \mathrm{~d}^{-1}\right)$ at $\sim 20 \mathrm{mg} / \mathrm{min}$ loading compared to $\sim 10 \mathrm{mg} / \mathrm{min}$ loading $\left(0.913 \mathrm{~d}^{-1}\right.$ for nickel, $1.03 \mathrm{~d}^{-1}$ for zinc). Lower rate coefficients for iron $\left(0.452 \mathrm{~d}^{-1}\right)$ and manganese $\left(0.891 \mathrm{~d}^{-1}\right)$ were observed at $\sim 20 \mathrm{mg} / \mathrm{min}$ loading, compared to $0.786 \mathrm{~d}^{-1}$ (iron) and $1.22 \mathrm{~d}^{-1}$ (manganese) observed at $\sim 10 \mathrm{mg} / \mathrm{min}$ loading.

A change from oxidizing $(>-50 \mathrm{mV})$ to reducing $(\leq-50 \mathrm{mV})$ conditions in the hydrosoil occurred in both the SSF and FWS series as O \& G loading increased from 10 to 20 $\mathrm{mg} / \mathrm{min}$. Wetland cells exposed to the greatest mass loading of $\mathrm{O} \& \mathrm{G}$ (i.e. cell 1 in both series) experienced the greatest decreases in reduction-oxidation potential through the duration of the experiment. The change from oxidizing to reducing conditions in the hydrosoil with increased mass loading occurring in both the SSF series (cells 1 \& 2) and FWS series (cells 1, 2, and 3) resulted in decreased removal efficiency of $O \& G$ from the water column. Removal of nickel and zinc was enhanced in both the SSF and FWS series with the change from oxidizing to reducing conditions coincident with increased mass loading. Results of this study demonstrate the importance of conditions, such as redox potential, in promoting biogeochemical processes for effective treatment in CWTSs and are applicable to the design and construction of full-scale systems. 
Table 64. Differences in treatment performance between hybrid CWTS and non-hybrid CWTS.

\begin{tabular}{clccc}
\hline Sampling Period & Parameter & Conc. $(\mathrm{mg} / \mathrm{L})$ & Removal $(\%)$ & Rate Coef. $\left(\mathrm{d}^{-1}\right)$ \\
\hline \multirow{2}{*}{1} & Influent & 97.2 & -- & -- \\
& Hybrid Extent & $<1.4$ & 98.5 & 1.13 \\
\multirow{2}{*}{2} & Influent & 101.2 & -- & -- \\
& Hybrid Extent & $<1.4$ & 98.6 & 0.872 \\
\multirow{2}{*}{3} & Influent & 102.1 & -- & -- \\
& Non-hybrid Extent & 9.9 & 90.3 & 0.583 \\
\multirow{2}{*}{4} & Influent & 103.5 & -- & - \\
& Non-hybrid Extent & 14.6 & 85.9 & 0.490 \\
\hline
\end{tabular}

Table 65. Redox potential $(\mathrm{mV})$ at different mass loadings of $\mathrm{O} \& \mathrm{G}$ for FWS and SSF series.

\begin{tabular}{|c|c|c|c|c|c|c|}
\hline & 1 & 2 & 3 & 4 & 5 & 6 \\
\hline O \& G-inflow (mg/L) & 98.7 & 96.9 & 101.2 & 99.3 & 103.5 & 98.6 \\
\hline \multicolumn{7}{|l|}{$\mathrm{O} \& \mathrm{G}$ loading $(\mathrm{mg} / \mathrm{min})$} \\
\hline FWS series & 10.28 & 10.10 & 10.55 & 10.35 & 21.57 & 20.55 \\
\hline SSF series & 8.91 & 8.75 & 9.14 & 8.97 & 18.69 & 17.81 \\
\hline \multicolumn{7}{|l|}{ Redox Potential (mV) } \\
\hline \multicolumn{7}{|l|}{ FWS series } \\
\hline Cell 1 & 98 & 69 & 37 & -91 & -110 & -147 \\
\hline Cell 2 & 20 & -30 & 51 & -10 & -51 & -91 \\
\hline Cell 3 & 49 & -15 & 43 & 19 & -67 & -49 \\
\hline Cell 4 & 72 & 83 & 87 & 75 & 65 & 72 \\
\hline \multicolumn{7}{|l|}{ SSF series } \\
\hline Cell 1 & 115 & 73 & -5 & 10 & -105 & -130 \\
\hline Cell 2 & 67 & -34 & 37 & -24 & -62 & -71 \\
\hline Cell 3 & -47 & -23 & 63 & 39 & 22 & -32 \\
\hline Cell 4 & 118 & 81 & 115 & 87 & 97 & 81 \\
\hline
\end{tabular}


Table 66. $O$ \& $\mathrm{G}$ removal extents, efficiencies, and rate coefficients for all 6 sampling periods at different loadings of $\mathrm{O} \& \mathrm{G}$.

\begin{tabular}{clrrrrrr}
\hline $\begin{array}{c}\text { Sampling } \\
\text { Period }\end{array}$ & Series & $\begin{array}{r}\text { O \& G } \\
\text { Loading } \\
(\mathrm{mg} / \mathrm{min})\end{array}$ & $\begin{array}{c}\text { HRT } \\
\text { (days) }\end{array}$ & $\begin{array}{c}\text { Inflow } \\
\text { Conc. } \\
(\mathrm{mg} / \mathrm{L})\end{array}$ & $\begin{array}{c}\text { Extent* } \\
(\mathrm{mg} / \mathrm{L})\end{array}$ & $\begin{array}{c}\text { Removal } \\
(\%)\end{array}$ & $\begin{array}{c}\text { Rate } \\
\text { Coef. } \\
\left(\mathrm{d}^{-1}\right)\end{array}$ \\
\hline 1 & SSF & 8.91 & 4 & 98.7 & 7.9 & 92.0 & 0.631 \\
& FWS & 10.28 & 4 & 98.7 & 5.5 & 94.4 & 0.722 \\
2 & SSF & 8.75 & 4 & 96.9 & 8.5 & 91.2 & 0.608 \\
& FWS & 10.10 & 4 & 96.9 & 6.7 & 93.1 & 0.668 \\
3 & SSF & 9.14 & 4 & 101.2 & 9.9 & 90.2 & 0.581 \\
& FWS & 10.55 & 4 & 101.2 & 8.1 & 92.0 & 0.631 \\
4 & SSF & 8.97 & 4 & 99.3 & 11.8 & 88.1 & 0.533 \\
& FWS & 10.35 & 4 & 99.3 & 14.6 & 85.3 & 0.479 \\
5 & SSF & 18.69 & 2 & 103.5 & 30.8 & 70.2 & 0.606 \\
& FWS & 21.57 & 2 & 103.5 & 29.8 & 71.2 & 0.623 \\
6 & SSF & 17.81 & 2 & 98.6 & 31.2 & 68.4 & 0.575 \\
& FWS & 20.55 & 2 & 98.6 & 35.3 & 64.2 & 0.514 \\
\hline
\end{tabular}

*Extent $=$ concentration in series outflow

Table 67. Metal removal extents, efficiencies, and rate coefficients for sampling periods 4 and 6 .

\begin{tabular}{|c|c|c|c|c|c|c|c|c|}
\hline Metal & $\begin{array}{l}\text { Sampling } \\
\text { Period }\end{array}$ & Series & $\begin{array}{c}\mathrm{O} \& \mathrm{G} \\
\text { Loading } \\
\text { (mg/min) }\end{array}$ & $\begin{array}{l}\text { HRT } \\
\text { (days) }\end{array}$ & $\begin{array}{l}\text { Inflow } \\
\text { Conc. } \\
\text { (mg/L) }\end{array}$ & $\begin{array}{l}\text { Extent* } \\
(\mathrm{mg} / \mathrm{L})\end{array}$ & $\begin{array}{c}\text { Removal } \\
(\%)\end{array}$ & $\begin{array}{l}\text { Rate Coef. } \\
\qquad\left(\mathrm{d}^{-1}\right)\end{array}$ \\
\hline \multirow[t]{4}{*}{ Nickel } & \multirow[t]{2}{*}{4} & SSF & 8.97 & 4 & 1.360 & 0.035 & 97.4 & 0.913 \\
\hline & & FWS & 10.35 & 4 & 1.360 & 0.220 & 83.8 & 0.455 \\
\hline & \multirow[t]{2}{*}{6} & SSF & 17.81 & 2 & 1.291 & 0.163 & 87.4 & 1.04 \\
\hline & & FWS & 20.55 & 2 & 1.291 & 0.417 & 67.7 & 0.565 \\
\hline \multirow[t]{4}{*}{ Zinc } & \multirow[t]{2}{*}{4} & SSF & 8.97 & 4 & 5.89 & 0.094 & 98.4 & 1.03 \\
\hline & & FWS & 10.35 & 4 & 5.89 & 0.103 & 98.3 & 1.01 \\
\hline & \multirow[t]{2}{*}{6} & SSF & 17.81 & 2 & 5.33 & 0.423 & 92.1 & 1.27 \\
\hline & & FWS & 20.55 & 2 & 5.33 & 0.611 & 88.5 & 1.08 \\
\hline \multirow[t]{4}{*}{ Iron } & \multirow[t]{2}{*}{4} & SSF & 8.97 & 4 & 0.431 & 0.019 & 95.7 & 0.786 \\
\hline & & FWS & 10.35 & 4 & 0.431 & 0.053 & 87.7 & 0.523 \\
\hline & \multirow[t]{2}{*}{6} & SSF & 17.81 & 2 & 0.420 & 0.170 & 59.5 & 0.452 \\
\hline & & FWS & 20.55 & 2 & 0.420 & 0.142 & 66.2 & 0.542 \\
\hline \multirow[t]{4}{*}{ Manganese } & \multirow[t]{2}{*}{4} & SSF & 8.97 & 4 & 1.314 & 0.010 & 99.2 & 1.22 \\
\hline & & FWS & 10.35 & 4 & 1.314 & 0.150 & 88.6 & 0.542 \\
\hline & \multirow[t]{2}{*}{6} & SSF & 17.81 & 2 & 1.202 & 0.202 & 83.2 & 0.891 \\
\hline & & FWS & 20.55 & 2 & 1.202 & 0.426 & 64.5 & 0.518 \\
\hline
\end{tabular}

*Extent $=$ concentration in series outflow 


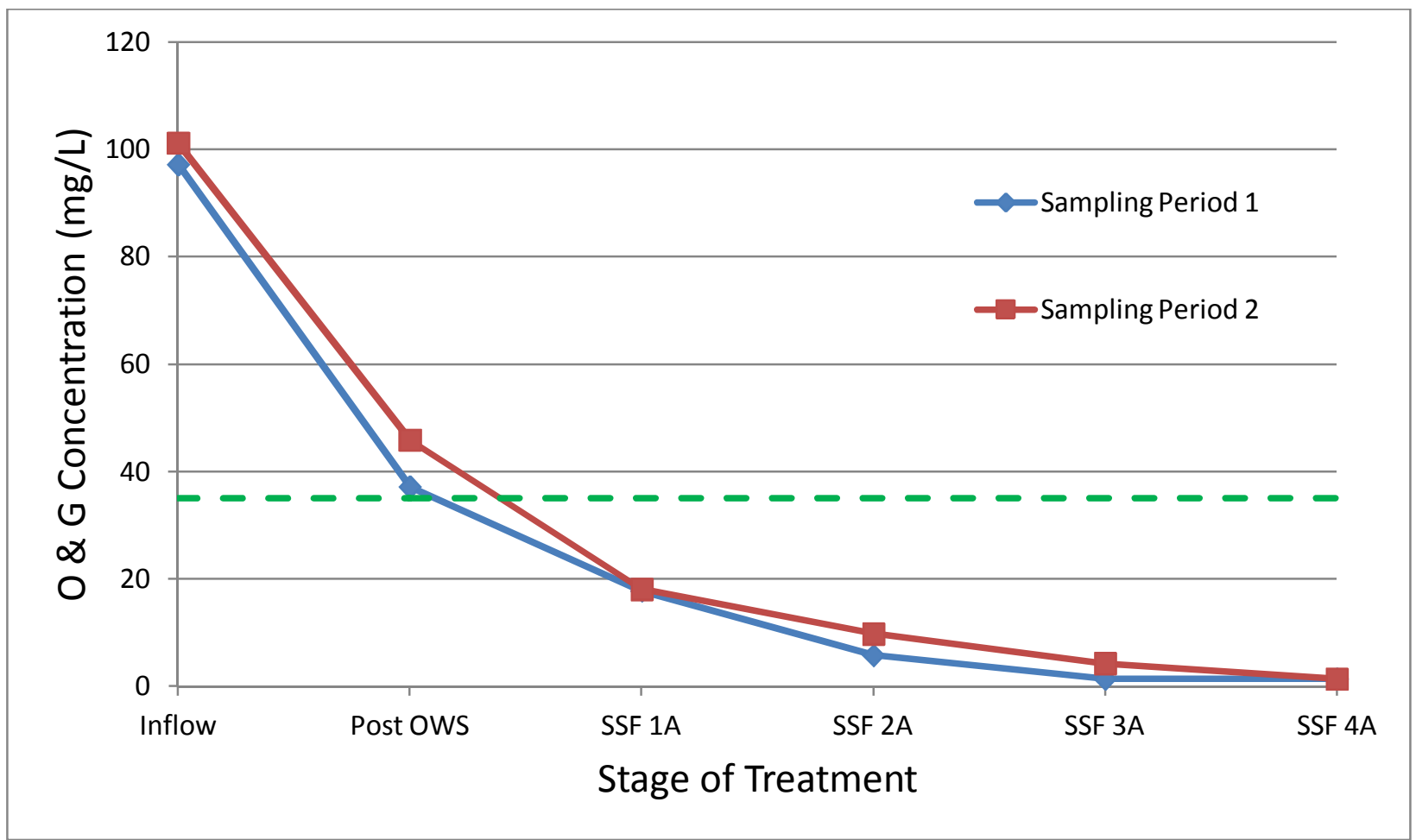

Figure 51. O \& G concentration during hybrid CWTS treatment with a goal of $35 \mathrm{mg} / \mathrm{L}$ (green dashed line). $35 \mathrm{mg} / \mathrm{L}=$ reuse criteria for irrigation and livestock watering.

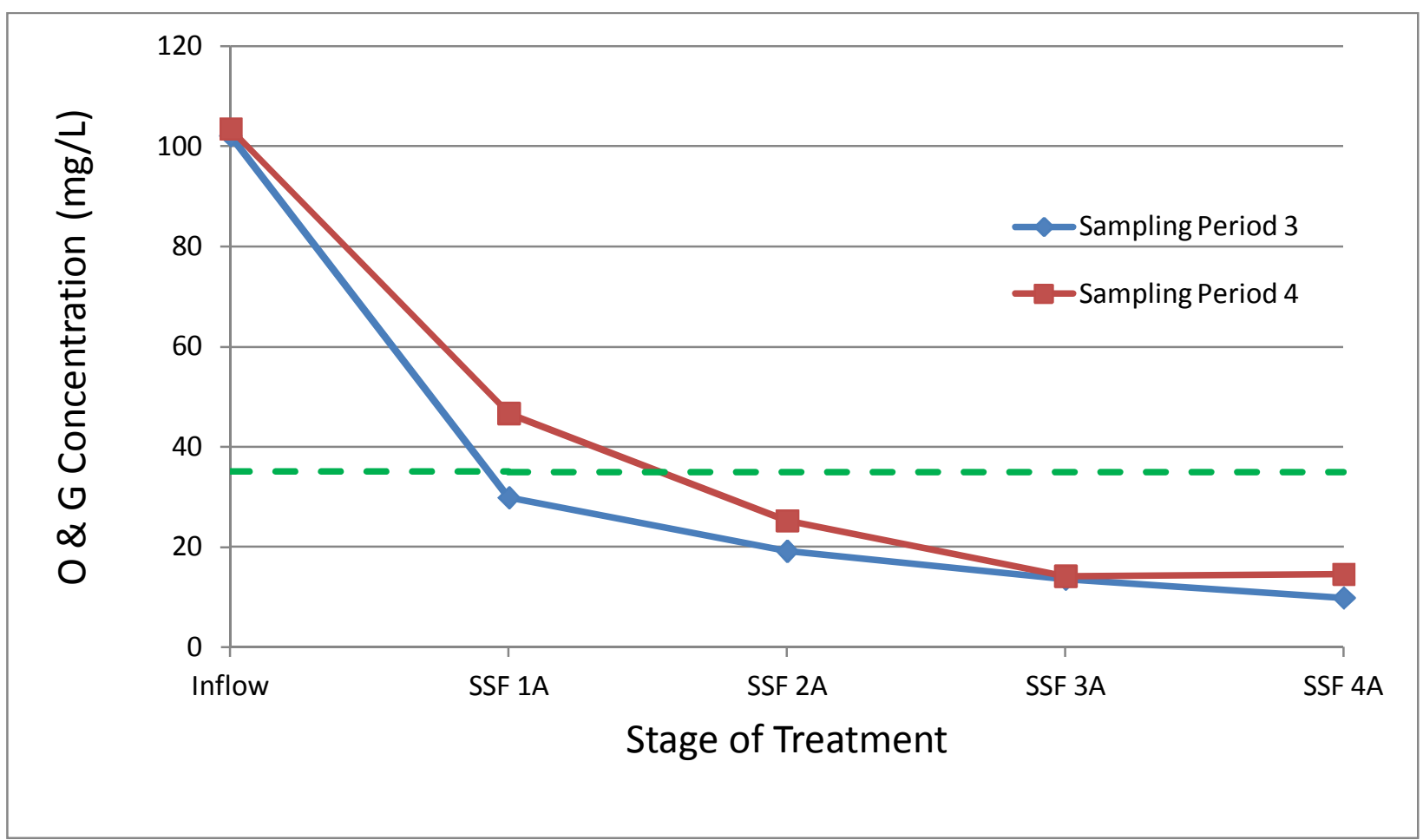

Figure 52. $\mathrm{O} \& \mathrm{G}$ concentration during non-hybrid CWTS treatment with a goal of 35 $\mathrm{mg} / \mathrm{L}$ (green dashed line). $35 \mathrm{mg} / \mathrm{L}=$ reuse criteria for irrigation and livestock watering. 


\section{Evapotranspiration and Tracer Tests}

Evapotranspiration serves as the single source of water loss in CWTSs through the combined effects of open water evaporation and plant transpiration. The decrease in flow associated with evapotranspiration can alter performance by enriching constituents and decreasing the system hydraulic retention time. In order to determine potential effects of evapotranspiration on treatment performance, it is necessary to determine the amount of water lost during treatment. For this reason, evapotranspiration was monitored in a pilotscale CWTS (post-RO control system) serving as a lysimeter and compared with reference evapotranspiration. Evapotranspiration was calculated using a simple mass balance in which the difference between the system inflow and outflow rates was assumed to be due to evapotranspiration. Inflow rates were measured using a stopwatch and graduated cylinder while outflow rates were measured using a digital rain gauge with an onboard data logger. Reference evapotranspiration was calculated with the FAO Penman Monteith equation (Allen et al, 1998) using data collected with an onsite weather station capable of recording temperature, wind speed and direction, barometric pressure, humidity, and solar radiation.

Results for measured CWTS evapotranspiration and reference evapotranspiration are shown in Figure 53. Results indicate a Typha latifolia (cattail) crop coefficient $\left(\mathrm{K}_{\mathrm{c}}\right)$ of 2.53 for data collected mid-summer during peak plant growth (Figure 54), which is within the range of published $\mathrm{K}_{\mathrm{c}}$ values measured using small stands of cattails (Snyder and Boyd, 1987; Rozkosny et al, 2006; Kadlec and Wallace, 2009). $\mathrm{K}_{\mathrm{c}}$ values are expected to change with seasonal variation in plant density and growth.

Theoretical lysimeter outflow determined using the Penman Monteith equation and midsummer peak $\mathrm{K}_{\mathrm{c}}$ is shown along with measured lysimeter outflow in Figure 55. This comparison graphically demonstrates application of the Penman Monteith equation to estimating evapotranspiration in the pilot-scale CWTSs used in this investigation. 


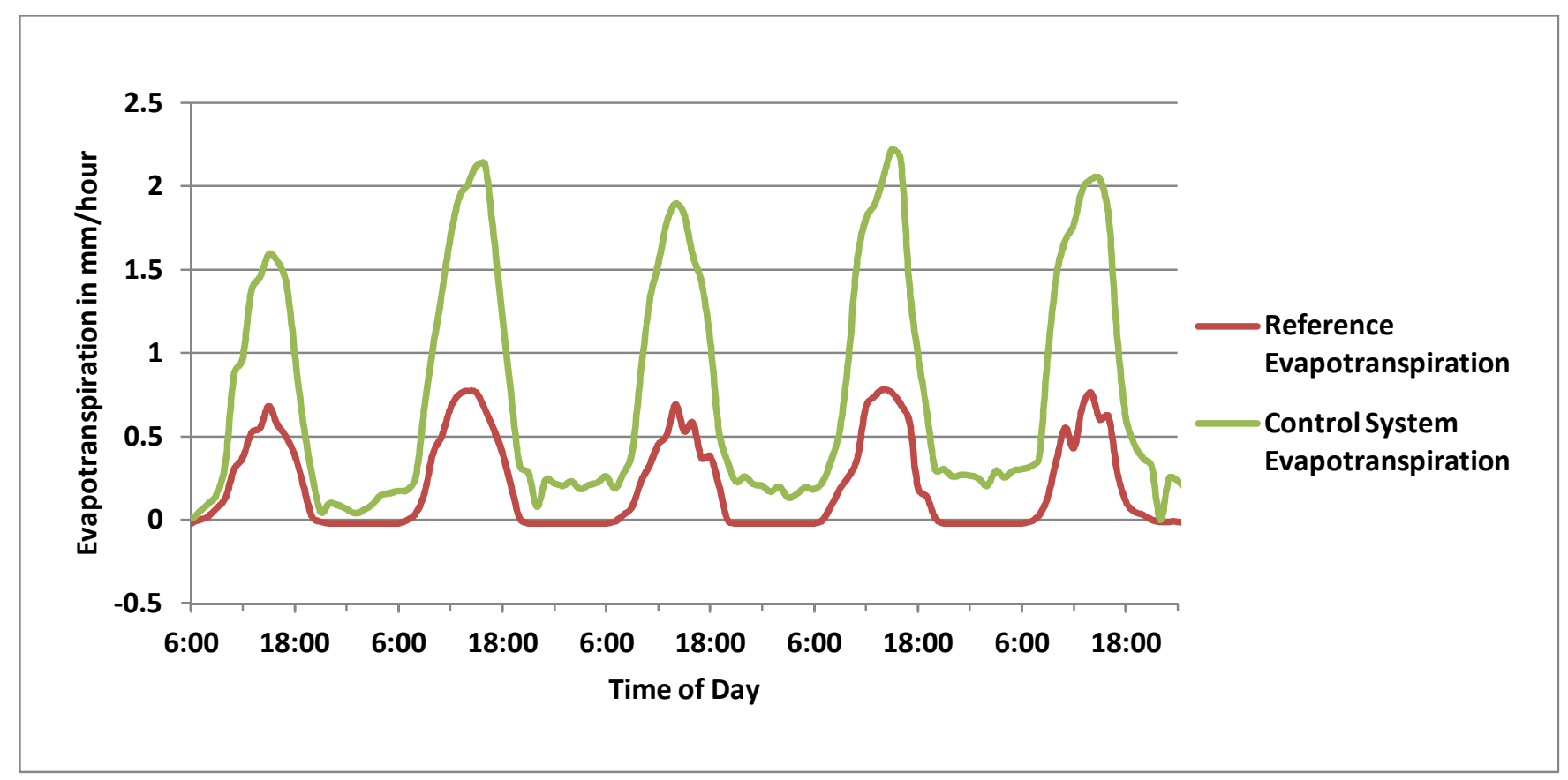

Figure 53. Hourly evapotranspiration monitoring using rain gauge data logger and on-site weather station.

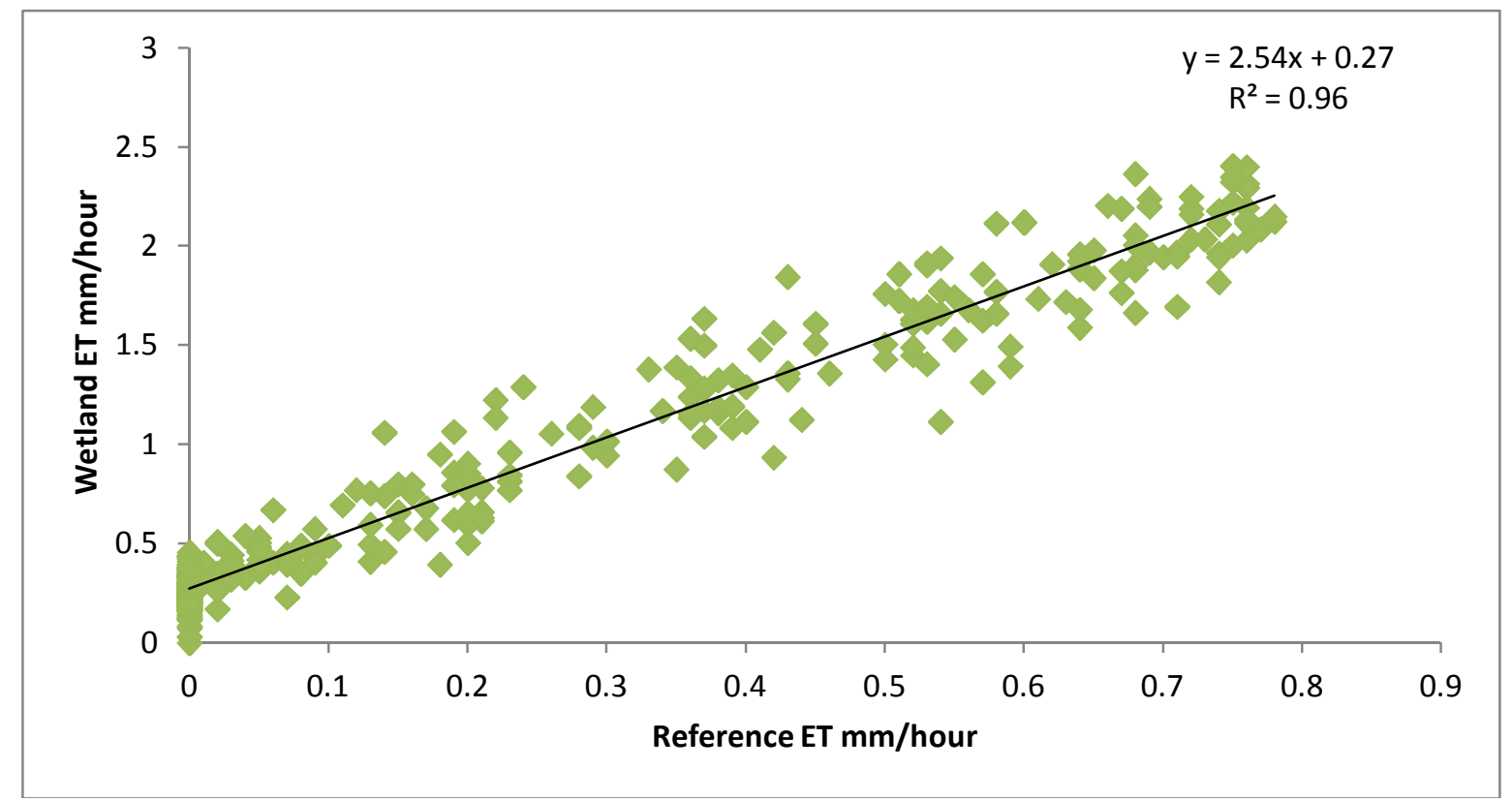

Figure 54. Determination of mid-summer $\mathrm{K}_{\mathrm{c}}$ using linear regression. System evapotranspiration can be estimated by multiplying reference evapotranspiration by $\mathrm{K}_{\mathrm{c}}$ (2.54) and adding the open water evaporation term (0.27). 


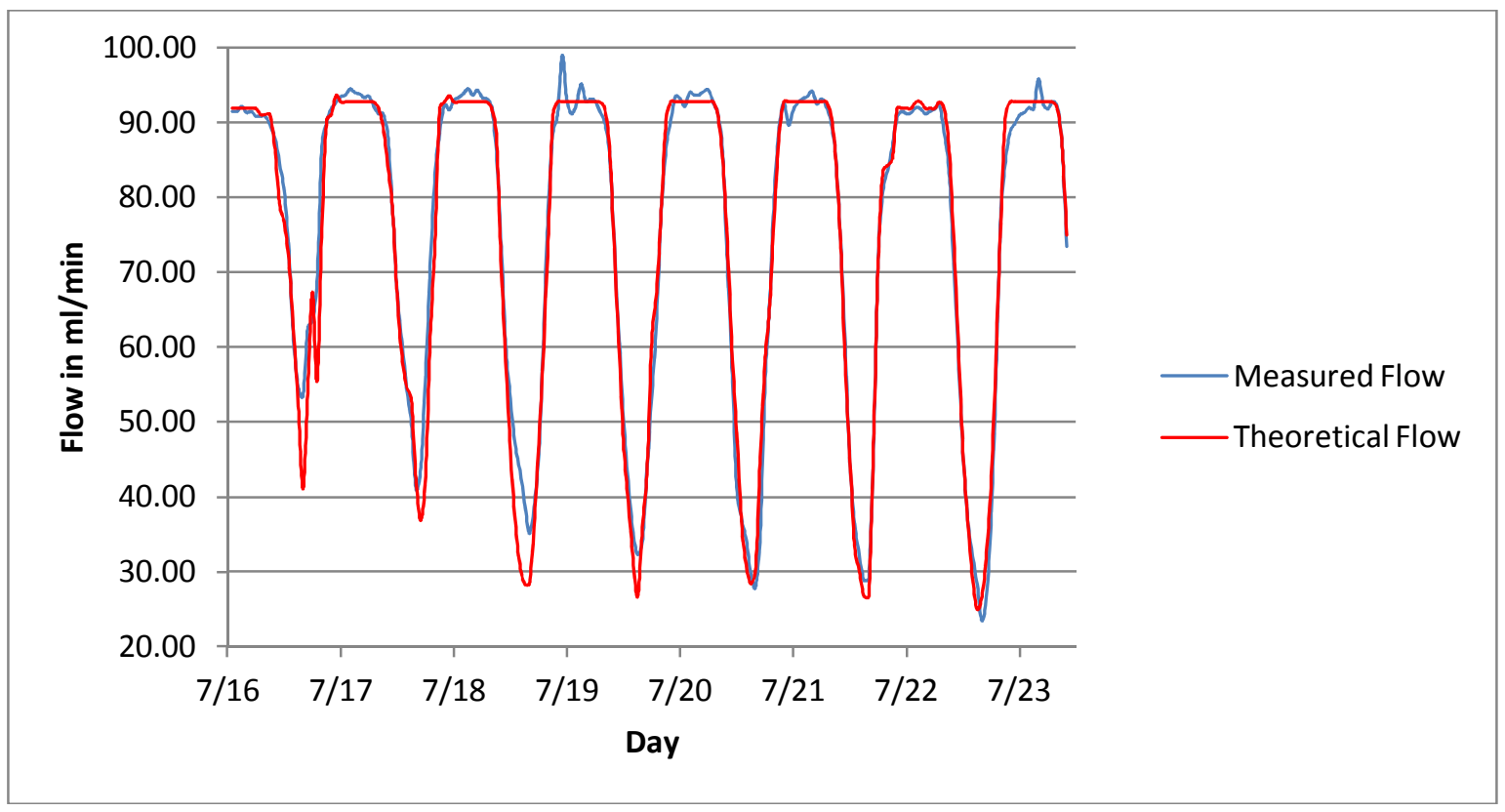

Figure 55. Comparison of theoretical lysimeter outflow vs. measured lysimeter outflow during mid-July showing application of the Penman Monteith equation for estimating water loss in pilot-scale CWTSs.

Plant transpiration may play a role in establishing a vertical hydraulic gradient within CWTS hydrosoil. This vertical hydraulic gradient may be a crucial component in initiating an advective flow path required for targeted constituents to reach the hydrosoil, where certain redox-driven reactions may occur (Kadlec, 1999; Martin et al., 2003; Kadlec and Wallace, 2009). For this reason, tracer tests were performed to determine the effect of evapotranspiration on vertical flux of constituents. Eight 20 -L buckets were prepared as bench-scale CWTSs by filling each with approximately $5 \mathrm{~L}$ of sandy sediment collected from 18-Mile Creek located near Clemson, SC and approximately $8 \mathrm{~T}$. latifolia plants. The systems were watered and fertilized regularly for a period of 9 months to allow for plant maturation. Surface water conductivity data collected from each system were used to formulate tracer solutions containing amounts of dissolved sodium chloride needed to yield conductivity ten times greater than that of the surface water in each system. This tracer solution concentration was selected to allow an accurate resolution of tracer arrival time.

Eight individual tracer tests were performed by placing a pair of stainless steel conductivity probes $5 \mathrm{~cm}$ below the surface of the hydrosoil of a system. Prepared tracer solution was then added by a FMI® QG400 pump at a rate of $200 \mathrm{~mL} / \mathrm{min}$. After 5 minutes, a second QG400 pumping at a rate of $200 \mathrm{~mL} / \mathrm{min}$ was connected to the system at a height of $25 \mathrm{~cm}$ above the hydrosoil to remove excess tracer solution, maintaining a constant head. This method allowed tracer solution to be added gently, preventing vertical flow disturbances while still allowing circulation of the tracer solution.

Soil conductivity was measured at 5-minute intervals until elevated conductivity was detected for a minimum of 3 consecutive measurements at each probe. T. latifolia in 4 
randomly selected systems was trimmed to a height of $35 \mathrm{~cm}$ above the hydrosoil (or $5 \mathrm{~cm}$ above the waterline) just prior to the tracer test. Mean arrival times for the planted and unplanted cells were compared using Welch's t-test to determine if plant transpiration significantly alters flow through the hydrosoil in the systems $(\alpha=0.05)$. Results indicated a significant difference $\left(\mathrm{p}=1.2 \times 10^{-8}\right)$ in tracer arrival time between the planted cells and the cells containing no plants (Figures 56 and 57) indicating that plant evapotranspiration can affect vertical transport of constituents.

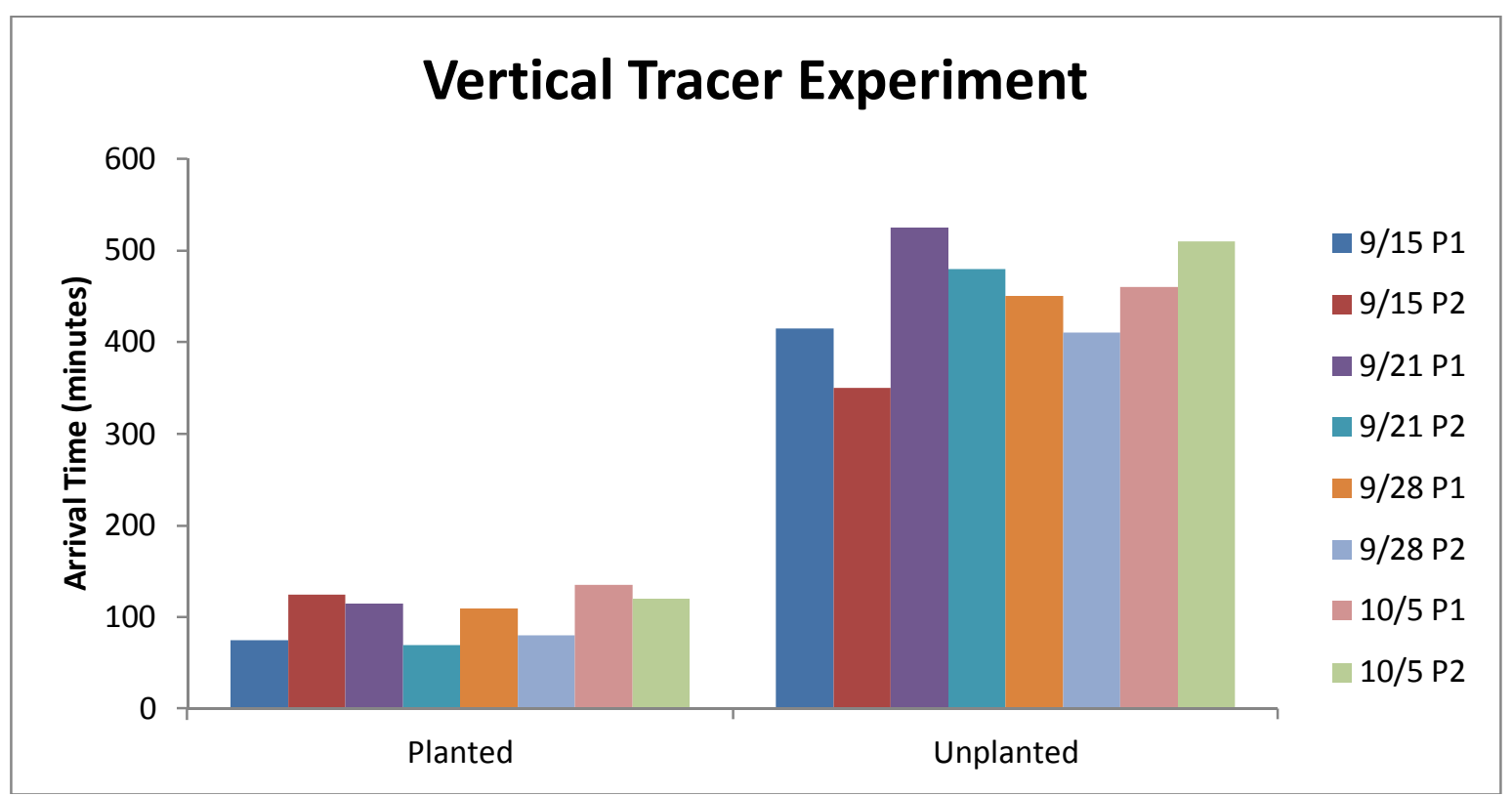

Figure 56. Arrival times for each probe during the 4 tracer tests performed on the planted systems and 4 on the unplanted systems. Lower arrival times in the planted systems indicate a more rapid transport of the tracer solution. 


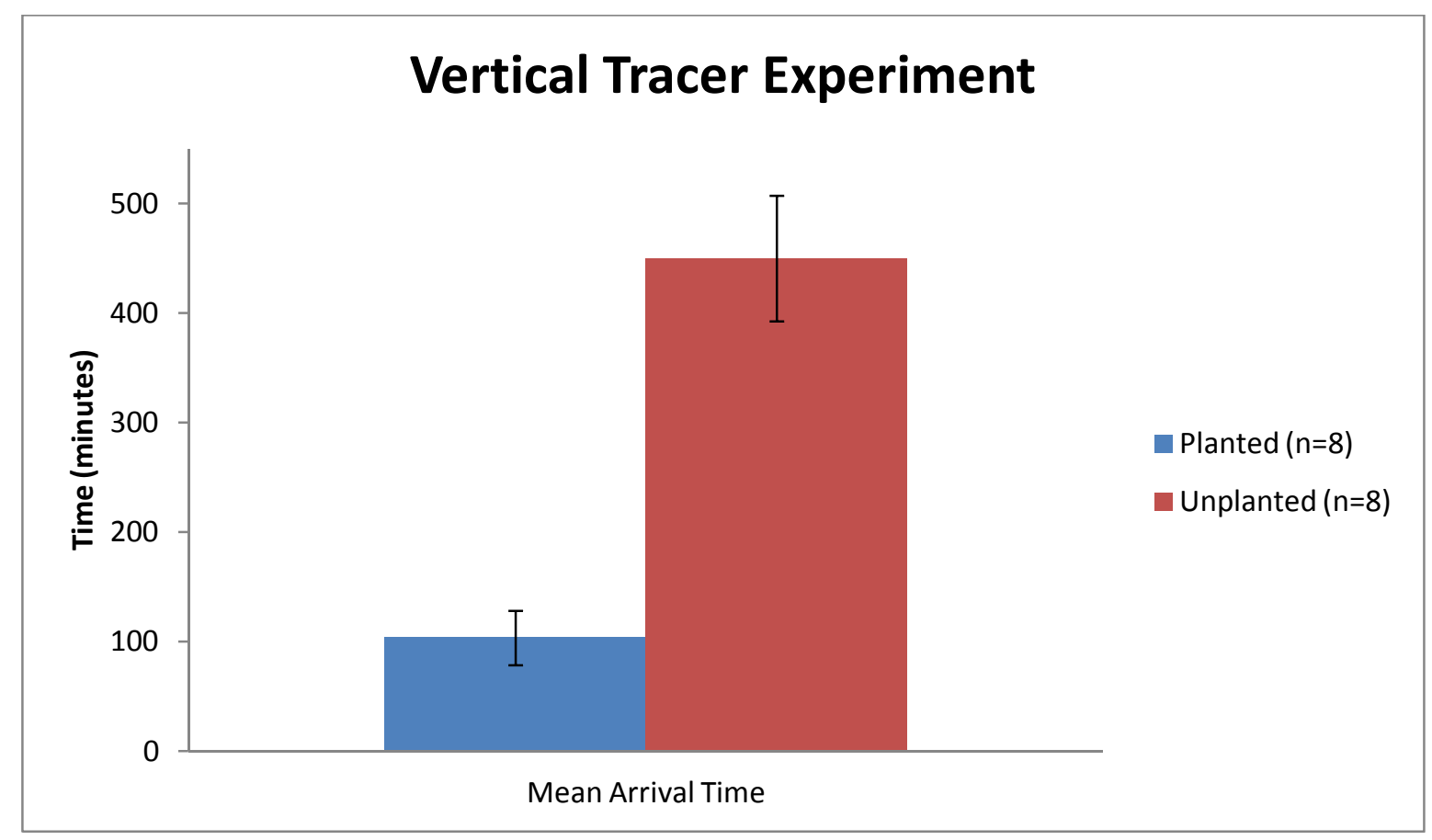

Figure 57. Mean arrival times of the 4 tracer tests performed on the planted systems and 4 on the unplanted systems. Error bars show standard deviation. The lower mean arrival time in the planted systems indicate a more rapid transport of the tracer solution.

\section{Design and Construction of Demonstration Wetland Treatment System}

A demonstration CWTS was designed and constructed to treat CBMPW from the Blue Creek field of the Black Warrior basin in central Alabama. Site-specific produced water samples were characterized to determine COCs targeted for treatment. Three water samples were collected from an on-site holding tank where produced water from multiple wells is stored prior to disposal (i.e. WS-20). The samples were analyzed using standard methods (APHA, 2005), and the results compared with surface water quality criteria from the Clean Water Act Section 304(a) to identify COCs. These criteria are recommended water quality criteria for the protection of aquatic life and human health in surface water for various beneficial uses. Comparison of analyzed constituents with water quality criteria (Table 68) yielded the following COCs: ammonia, barium, cadmium, iron, manganese, nitrate, and selenium. Because results indicate that composition of the produced water analyzed varies with time, the demonstration CWTS was designed to promote conditions required for treatment of both site-specific COCs identified from the water samples analyzed and additional generic COCs identified in produced waters characterized in Task 2. Conditions required for removal of ammonia, cadmium, iron, manganese, nitrate, oil and grease, and selenium identified during Phase II (Tables 20-23, 58, 61, and 62) were targeted in designing the demonstration system. Oxidizing conditions (DO greater than 2.0 $\mathrm{mg} / \mathrm{L}$ ) were targeted in some of the demonstration CWTS cells to promote microbial nitrification of ammonia, biodegradation of oil and grease, and precipitation of iron and 
manganese. Reducing conditions (DO less than $2.0 \mathrm{mg} / \mathrm{L}$ ) were targeted in the remaining cells to promote microbial denitrification of nitrate, sulfide complexation with divalent metals through dissimilatory sulfate reduction, and dissimilatory selenium reduction. Barium was not targeted for pilot-scale treatment during Phase II; however, removal through chemical precipitation can occur under the conditions observed ( $\mathrm{pH}$ between 2 and 12) in the pilot-scale CWTSs when sulfate is present (Brookings, 1988).

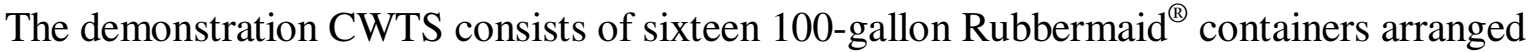
in four series with four containers as wetland cells in each series. Each cell was filled to a depth of approximately $25 \mathrm{~cm}$ with sand and then planted with cattails (Typha latifolia and Typha angustifolia) collected from a pond located near the site. Connections between cells were made using $2.5-\mathrm{cm}$ poly-vinyl chloride (PVC) pipes to allow gravity flow through each series. Water is pumped from a 5,678-L polypropylene carboy holding tank to the first cell in each series by $\mathrm{FMI}^{\circledR}$ piston pumps. Each pump was calibrated for a hydraulic retention time of 24 hours per cell. The 24-hour HRT was selected based on rate coefficients determined from the pilot-scale experiments. The outflow pipes from each cell were connected to a small basin, from which a sump pump moves the outflow water to a second 5,678-L polypropylene carboy holding tank for return to the existing waste stream (Figures 58-61).

The demonstration CWTS contains two pairs of replicated series, each pair receiving different hydrosoil amendments. Dried yeast in the form of AquaSmart ${ }^{\mathrm{TM}}$ was added to the hydrosoil of cells 2 and 3 of the first two series (series A and B; approximately $10 \mathrm{~kg}$ per cell) to serve as a source of organic carbon to promote reducing conditions and to provide nutrients for both the plants and microbes. Shredded hardwood mulch (approximately 10 $\mathrm{kg}$ per cell) was added to the hydrosoil of cells 2 and 3 of the second two series (series $\mathrm{C}$ and D) to serve as a source of organic carbon and promote reducing conditions. Cells 1 and 4 in each of the four series were designed as oxidizing cells and therefore did not receive any organic amendment. The oxidizing and reducing cells are capable of treating constituents that are not currently identified as COCs, but that may be present in the future at concentrations exceeding water quality criteria. Municipal (fresh) water was introduced initially to the wetland series to allow for growth of plants and microbes prior to introduction of produced water. 
Table 68. Characterization of untreated CBMPW samples collected from the WS-20 tank at demonstration site and surface water quality criteria. Constituent concentrations are $\mathrm{mg} / \mathrm{L}$ unless noted.

\begin{tabular}{|c|c|c|c|c|}
\hline Parameters ${ }^{1}$ & 6/7/2011 Sample & 5/29/2012 Sample & 6/11/2012 Sample & Water Quality Criteria $^{2}$ \\
\hline Conductivity $(\mu \mathrm{m} / \mathrm{cm})$ & 7860 & 11100 & 6950 & NA \\
\hline $\mathrm{pH}(\mathrm{S} . \mathrm{U})$. & 8.49 & 7.98 & 8.35 & NA \\
\hline Alkalinity $\left(\right.$ as $\left.\mathrm{CaCO}_{3}\right)$ & 496 & 406 & 450 & NA \\
\hline Hardness (as $\mathrm{CaCO}_{3}$ ) & 226 & 504 & 468 & NA \\
\hline Ammonia (as N) & 2.8 & 2.1 & 2.0 & 1.4 \\
\hline Nitrate & NM & 12.2 & NM & 10 \\
\hline Oil and Grease & $<2.8$ & $<2.8$ & NM & 10 \\
\hline Sulfate & NM & 4.6 & $\mathrm{NM}$ & NA \\
\hline Total Dissolved Solids & NM & 6420 & 6850 & NA \\
\hline Total Suspended Solids & NM & 10 & 10 & NA \\
\hline $\mathrm{Al}$ & $<0.045$ & 0.130 & $<0.045$ & NA \\
\hline As & 0.00074 & $<0.053$ & $<0.053$ & 0.15 \\
\hline $\mathrm{Ba}$ & 29.6 & 13.8 & 11.0 & 1.0 \\
\hline $\mathrm{Be}$ & $<0.0003$ & NM & $<0.0003$ & 0.004 \\
\hline $\mathrm{Br}$ & NM & 6.80 & NM & NA \\
\hline $\mathrm{Ca}$ & 93.6 & 72.2 & 62.7 & NA \\
\hline $\mathrm{Cd}$ & $<0.0034$ & $<0.001$ & 0.0315 & 0.00025 \\
\hline $\mathrm{Cl}$ & NM & 3740 & NM & NA \\
\hline Co & $<0.007$ & NM & $<0.007$ & NA \\
\hline $\mathrm{Cr}$ & $<0.0061$ & $<0.0061$ & $<0.0061$ & 0.011 \\
\hline $\mathrm{Cu}$ & $<0.003$ & $<0.003$ & $<0.003$ & 0.009 \\
\hline $\mathrm{Fe}$ & $<0.0062$ & 0.35 & 1.6 & 1.0 \\
\hline $\mathrm{Hg}$ & $<0.0025$ & NM & NM & NA \\
\hline $\mathrm{Mg}$ & 53.7 & 29.0 & 28.0 & NA \\
\hline $\mathrm{Mn}$ & $<0.0014$ & 0.06 & $<0.0014$ & 0.05 \\
\hline $\mathrm{Na}$ & NM & 1900 & 25.6 & NA \\
\hline $\mathrm{Ni}$ & $<0.015$ & 0.01 & $<0.015$ & 0.61 \\
\hline $\mathrm{Pb}$ & $<0.042$ & $<0.0015$ & $<0.0015$ & 0.0025 \\
\hline $\mathrm{Se}$ & 0.0032 & 0.019 & NM & 0.005 \\
\hline $\mathrm{Sr}$ & 6.81 & NM & 2.08 & NA \\
\hline V & $<0.0075$ & NM & $<0.0075$ & NA \\
\hline $\mathrm{Zn}$ & $<0.0018$ & 0.020 & $<0.0018$ & 0.12 \\
\hline
\end{tabular}

${ }^{1}$ Metal concentrations are acid soluble concentrations.

${ }^{2}$ Current water quality criteria from the Clean Water Act Section 304(a), chronic toxicity values used where available NA - No suggested criteria from Clean Water Act Section 304(a)

NM - Not measured. 


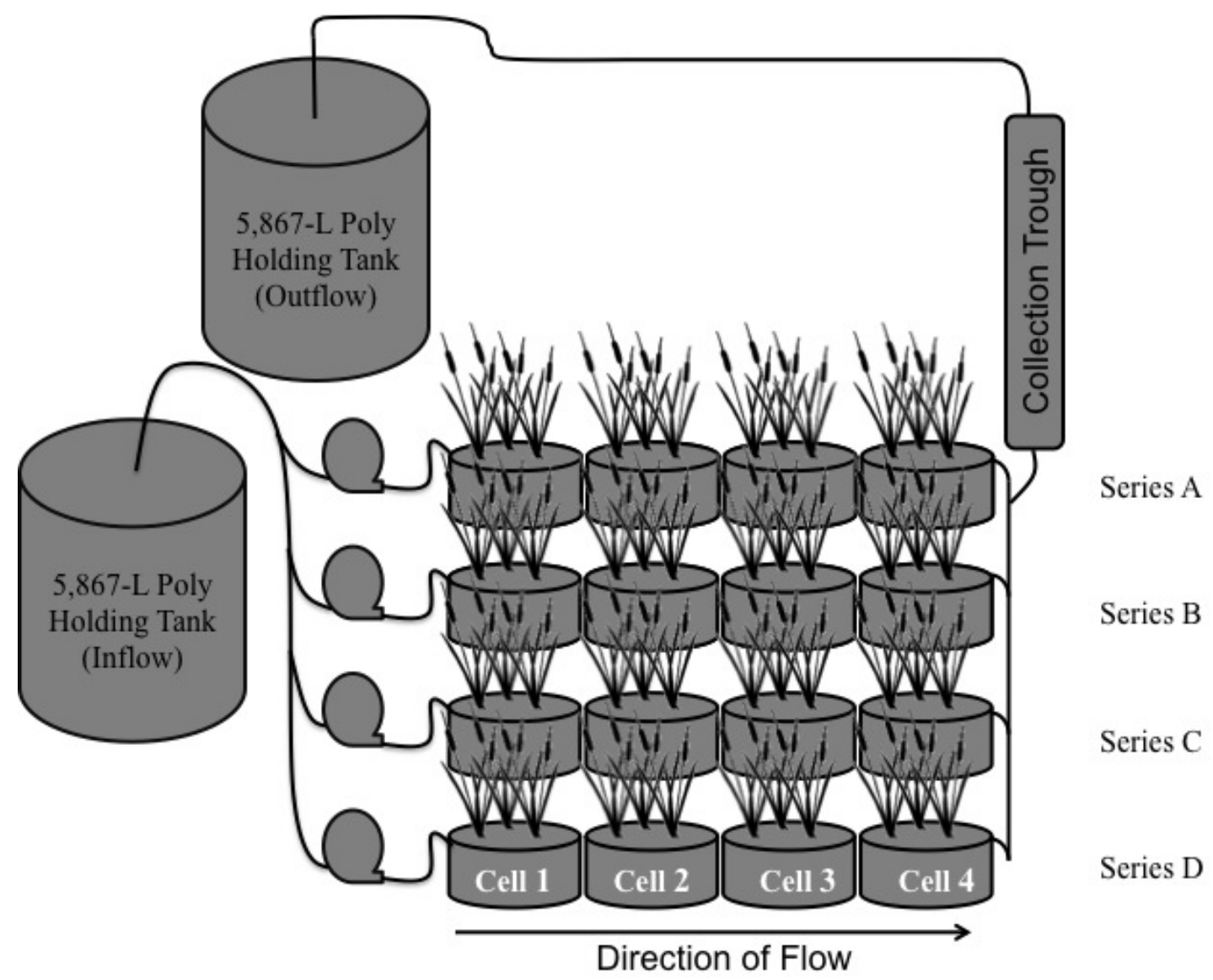

Figure 58. Schematic diagram of demonstration CWTS. Produced water is pumped from the 5,867-L polypropylene carboy holding tank through the 4 series to an outflow holding tank. Cells of each series are arranged for gravity flow. Cells 1 and 4 of each series were designed to promote oxidizing conditions, and cells 2 and 3 were designed to promote reducing conditions with the addition of AquaSmart ${ }^{\mathrm{TM}}$ (series A and B) or mulch (series $\mathrm{C}$ and D). 


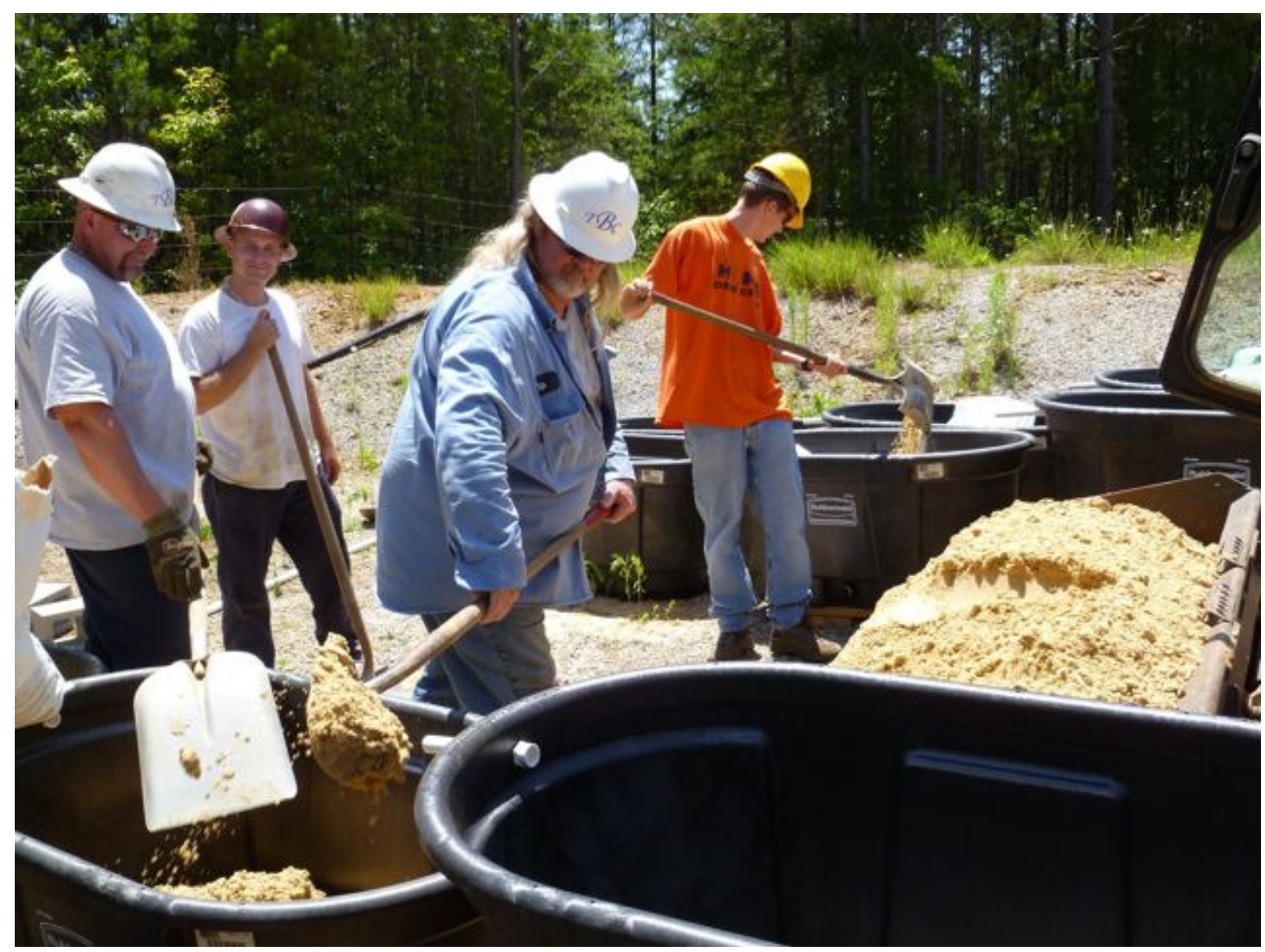

Figure 59. Filling the demonstration CWTS cells with sand. 


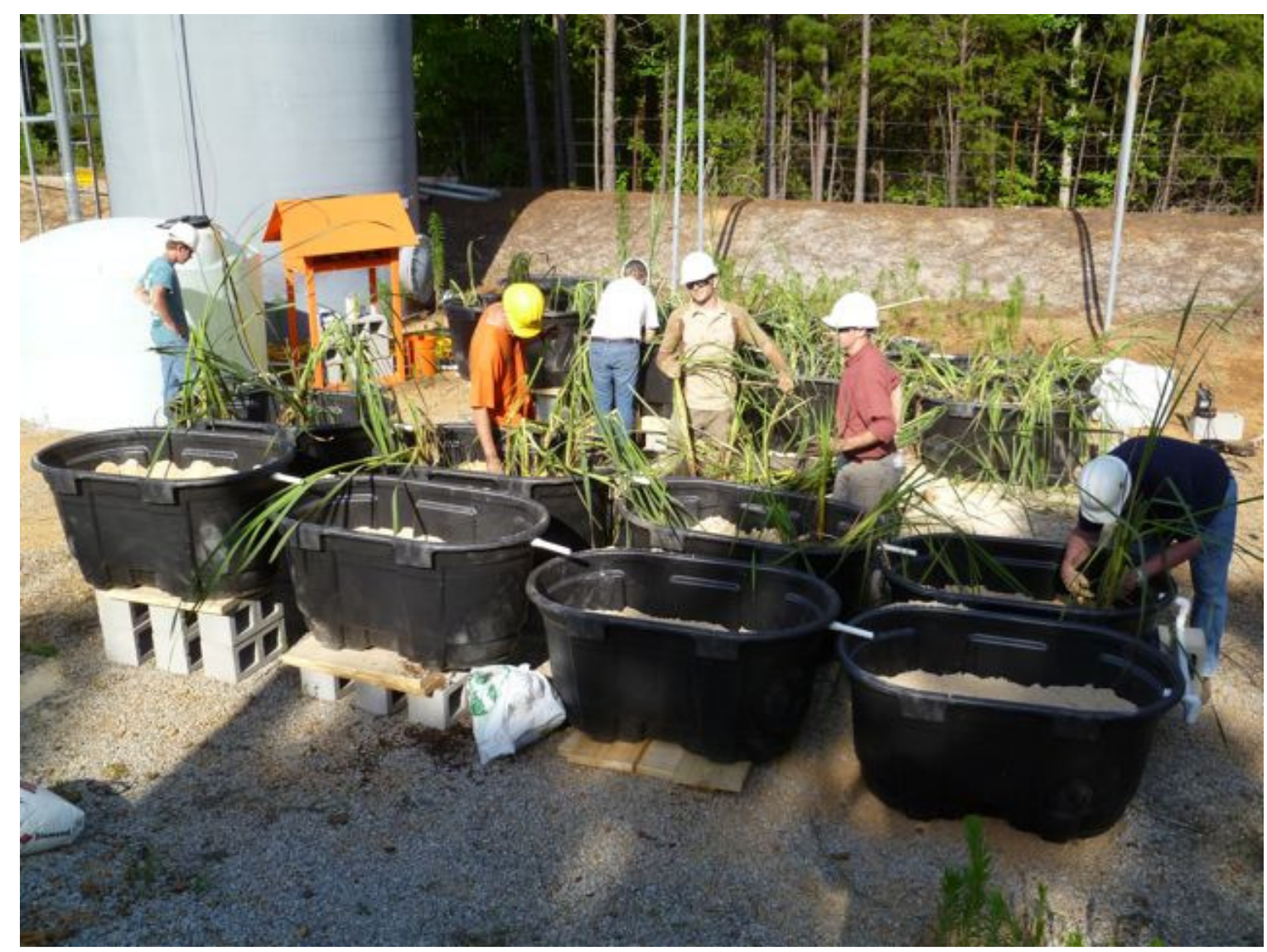

Figure 60. Broadleaf and narrowleaf cattails (Typha latifolia and Typha angustifolia) were collected from a nearby pond and transplanted to the demonstration CWTS cells at a density of 30 plants per cell. 


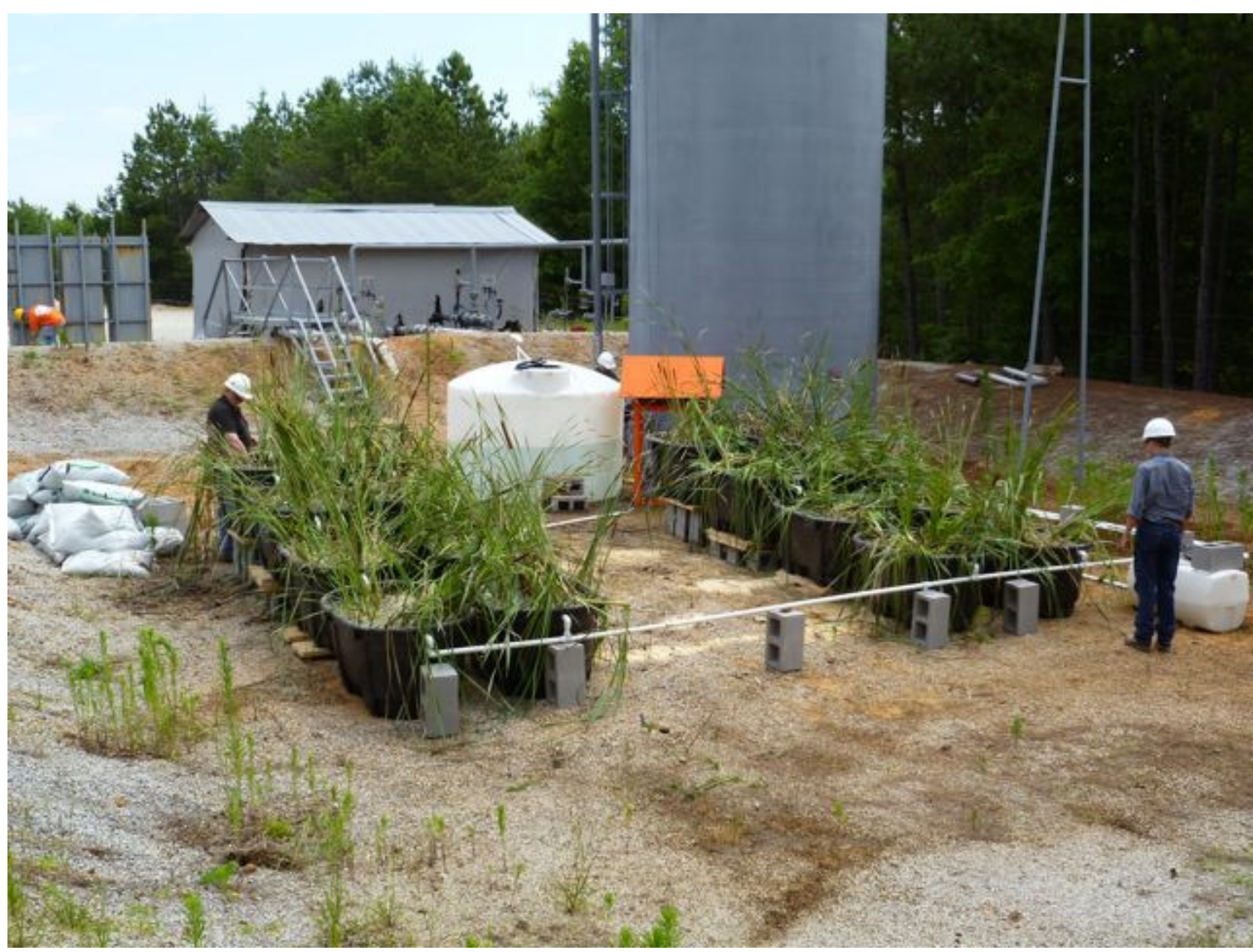

Figure 61. Demonstration CWTS after planting was completed on 5/31/12. Municipal water was introduced initially to the four series to allow growth and maturation of the plants and microbes. 
Measure Treatment Performance of Demonstration Constructed Wetland System (Task 8)

Produced water was introduced to the system on September 3, 2012 (14 weeks after construction), and samples were collected from the inflow and outflow of each cell on 9/6/12, 9/20/12, 10/4/12, 10/18/12, 11/1/12, 11/15/12, 11/29/12, 12/13/12, 1/10/13, $1 / 24 / 13,2 / 7 / 13,2 / 21 / 13,3 / 14 / 13$, and $3 / 28 / 13$ for a total of fourteen sampling periods, numbered 1 through 14, respectively. Samples from were analyzed for various constituents (Table 2) and explanatory parameters (Tables 69-82). Of the COCs identified initially in design of the demonstration wetland treatment system, ammonia, $\mathrm{Ba}, \mathrm{Cd}, \mathrm{Fe}$, and Mn concentrations in the PW inflow exceeded the water quality criteria (WQC) during one or more sampling periods. Se and nitrate concentrations did not exceed WQC during any of the sampling periods.

Removal efficiencies, removal rate coefficients, and removal extents of ammonia, $\mathrm{Ba}, \mathrm{Cd}$, $\mathrm{Fe}$, and $\mathrm{Mn}$ in the demonstration system were calculated (Tables 83-87) to determine efficiency and effectiveness of the design for each cell, which was based on biogeochemical treatment processes. Removal efficiency was calculated using the following equation:

$$
\text { Removal efficiency }(\%)=\left(\frac{[C]_{0}-[\mathrm{C}]}{[C]_{0}}\right) \times 100 \quad \text { Eqn. } 10
$$

where $[\mathrm{Co}]$ is concentration $(\mathrm{mg} / \mathrm{L})$ of a $\mathrm{COC}$ in the inflow and $[C]$ is concentration $(\mathrm{mg} / \mathrm{L})$ in the outflow. Removal rate coefficients were calculated using the standard approach of first-order rate kinetics (Horner et al. 2012; Johnson et al. 2008b). The firstorder reaction follows:

$$
k=\frac{-\ln \left([C] /[C]_{0}\right)}{t}
$$

where $k$ is the rate coefficient $\left(\mathrm{day}^{-1}\right),[C o]$ is the initial inflow COC concentration, $[C]$ is the final outflow COC concentration, and $t$ (days) is the HRT for the system. Comparing treatment goals with calculations of performance parameters will assess success of the demonstration system.

Ammonia concentration was less than the WQC (1.4 mg/L) in both inflow and outflow during the first three sampling periods (Tables 69-71). During the subsequent sampling periods (Tables 72-82), ammonia was present in concentrations above the WQC, and concentrations decreased in each series to less than the WQC (Figure 62, Table 83). Nitrate concentration was measured in samples collected only during sampling period 6 (Figure 63), and the nitrate concentrations measured in the outflow were greater than what would be produced if all the ammonia in the inflow were converted to nitrate. This indicates that the ammonia in the inflow was being removed as well as additional ammonia produced in the system.

During sampling periods 1 through 5, Ba concentration in outflow was less than the WQC of $1.0 \mathrm{mg} / \mathrm{L}$ (Figures 64, Tables 69-73). Over $90 \%$ of the inflow concentration of Ba was 
removed during each of these sampling periods (1 through 5) (Table 84). During sampling periods 6 and 7 (Tables 74 and 75), no Ba removal occurred, which was interpreted as due to a lack of sulfate in the wetland cells (Figure 65, Table 84). Sulfate concentration measured during sampling period 6 indicated that the amount of sulfate available was insufficient for decreasing Ba concentrations. Gypsum was added to all wetland cells during sampling period 7 on 11/29/12 as a source of sulfate. After the addition of gypsum, removal increased during sampling periods 8-10 (Figure 66, Table 84).

Cd concentrations decreased to below WQC $(0.00025 \mathrm{mg} / \mathrm{L})$ from the produced water by treatment in the wetland cells during the first two sampling periods (Figure 67, Table 85). After the second sampling period, $\mathrm{Cd}$ was not present in the $\mathrm{PW}$ in concentrations greater than the WQC.

Fe concentration was less than the targeted performance goal of $1.0 \mathrm{mg} / \mathrm{L}$ in both inflow and outflow for sampling periods 1-3 and 7-14 (Tables 69-71 and 75-82). During sampling periods 4,5 , and 6, Fe concentration in the inflow was greater than $1.0 \mathrm{mg} / \mathrm{L}$ with decrease in concentration from inflow to outflow in all series (Figure 68, Table 86).

Mn concentration exceeded the WQC $(0.05 \mathrm{mg} / \mathrm{L})$ in inflow during the first sampling period, and no removal occurred in the wetland cells (Table 69 and 87). Decrease in concentrations of Mn occurred during the second sampling period in series B and C (Table 70 and 87). During sampling period 4, Mn was present in the inflow and decreased in concentration to below WQC in all wetland series (Table 72). Mn concentration was below the WQC in sampling periods 3, 5-7, and 11-12. During sampling periods 8-10, Mn concentrations were below the WQC of $0.05 \mathrm{mg} / \mathrm{L}$ in inflow and outflow, and removal occurred in all series (Tables 76-78 and 87). During sampling periods 13-14, inflow Mn concentration was 0.0659 and $0.0771 \mathrm{mg} / \mathrm{L}$, respectively, and decreased to below WQC in all series.

Results indicate that the demonstration CWTS can reduce concentrations of COCs as they vary through time. The system successfully achieved consistent removal extents although inflow concentrations differed by orders of magnitude. Plant growth (Figure 69) and explanatory parameters measured in the wetland treatment cells during all sampling periods were favorable for decreasing concentrations of COCs in the produced water, with the exception of Ba during sampling periods 6 and 7 as discussed above. After the addition of gypsum to all wetland cells, Ba removal increased during the remaining sampling periods, indicating that the targeted concentration can be achieved by properly adjusting wetland conditions. Upon the conclusion of sampling to measure treatment performance, the demonstration system was disassembled on May 6, 2013. 
Table 69. COC concentrations and explanatory parameters for Sampling Period 1 (9/6/12)

\begin{tabular}{|c|c|c|c|c|c|c|c|c|c|c|c|c|}
\hline \multirow[b]{2}{*}{$\begin{array}{l}\text { Sampling } \\
\text { Location }\end{array}$} & \multicolumn{5}{|c|}{ Performance Parameters } & \multicolumn{7}{|c|}{ Explanatory Parameters } \\
\hline & $\begin{array}{c}\text { Ammonia } \\
(\mathrm{mg} / \mathrm{L})\end{array}$ & $\begin{array}{c}\text { Barium } \\
(\mathrm{mg} / \mathrm{L})\end{array}$ & $\begin{array}{c}\text { Iron } \\
(\mathrm{mg} / \mathrm{L})\end{array}$ & $\begin{array}{c}\text { Manganese } \\
(\mathrm{mg} / \mathrm{L})\end{array}$ & $\begin{array}{c}\text { Cadmium } \\
(\mathrm{mg} / \mathrm{L})\end{array}$ & $\begin{array}{c}\mathrm{DO} \\
(\mathrm{mg} / \mathrm{L})\end{array}$ & $\begin{array}{c}\text { Cond. } \\
(\mu \mathrm{S} / \mathrm{cm})\end{array}$ & $\begin{array}{c}\mathrm{pH} \\
\text { (S.U.) }\end{array}$ & $\begin{array}{c}\text { Redox } \\
(\mathrm{mV})\end{array}$ & $\begin{array}{c}\text { Temp } \\
\left({ }^{\circ} \mathrm{C}\right)\end{array}$ & $\begin{array}{c}\text { Alkalinity } \\
(\mathrm{mg} / \mathrm{L} \text { as } \\
\left.\mathrm{CaCO}_{3}\right)\end{array}$ & $\begin{array}{l}\text { Hardness } \\
(\mathrm{mg} / \mathrm{L} \text { as } \\
\left.\mathrm{CaCO}_{3}\right) \\
\end{array}$ \\
\hline $\mathrm{A} 1^{\mathrm{a}}$ & 1.1 & 6.4 & 0.2201 & 1.114 & 0.000032 & 2.85 & 6570 & 7.58 & -137 & 26.4 & 420 & 1620 \\
\hline $\mathrm{A} 2$ & 1.5 & 3.5 & 0.5943 & 1.333 & $<0.000025$ & 1.3 & 4490 & 7.69 & -233 & 27.2 & 320 & 1800 \\
\hline A3 & 1.6 & 1.4 & 0.8435 & 1.503 & $<0.000025$ & 2.34 & 2608 & 7.02 & -325 & 26.5 & 130 & 1260 \\
\hline B1 & 0.5 & 8.1 & 0.4869 & 0.7397 & 0.000037 & 3.33 & 6690 & 6.86 & 61 & 27 & 208 & 1620 \\
\hline $\mathrm{B} 2$ & 0.4 & 3.9 & 0.6422 & 1.204 & 0.000031 & 4.02 & 4386 & 6.99 & -201 & 26.4 & 156 & 1260 \\
\hline B3 & 0.4 & 1.2 & 0.3977 & 3.082 & $<0.000025$ & 3.6 & 2630 & 7.02 & -94 & 25.8 & 114 & 1440 \\
\hline B4 & 0.6 & 0.13 & 0.3139 & 2.658 & 0.000034 & 4.1 & 1354 & 6.65 & 151 & 25.6 & 78 & 1080 \\
\hline $\mathrm{C} 1$ & 0.5 & 6.5 & 0.1494 & 1.11 & 0.000154 & 2.3 & 6690 & 7.45 & 142 & 27.6 & 198 & 1620 \\
\hline D2 & 0.4 & 2.7 & 0.0375 & 1.113 & 0.000033 & 2.29 & 4601 & 6.98 & -142 & 25.7 & 162 & 1620 \\
\hline D3 & 0.4 & 0.55 & 0.0783 & 1.874 & 0.00004 & 1.59 & 2565 & 6.97 & -232 & 26.7 & 70 & 1080 \\
\hline D4 & 0.3 & 0.21 & 0.1755 & 1.019 & 0.000107 & 1.12 & 1354 & 6.96 & 178 & 26.4 & 64 & 900 \\
\hline
\end{tabular}

nd- not determined
Se below detection limit ( $b d l)(0.0002 \mathrm{mg} / \mathrm{L})$.

a Sampling location format is series (A), cell (1), etc., (Figure 1). 
Table 70. COC concentrations and explanatory parameters for Sampling Period $2(9 / 20 / 12)$

\begin{tabular}{|c|c|c|c|c|c|c|c|c|c|c|c|c|}
\hline \multirow[b]{2}{*}{$\begin{array}{l}\text { Sampling } \\
\text { Location }\end{array}$} & \multicolumn{5}{|c|}{ Performance Parameters } & \multicolumn{7}{|c|}{ Explanatory Parameters } \\
\hline & $\begin{array}{c}\text { Ammonia } \\
(\mathrm{mg} / \mathrm{L})\end{array}$ & $\begin{array}{c}\text { Barium } \\
(\mathrm{mg} / \mathrm{L})\end{array}$ & $\begin{array}{c}\text { Iron } \\
(\mathrm{mg} / \mathrm{L})\end{array}$ & $\begin{array}{c}\text { Manganese } \\
(\mathrm{mg} / \mathrm{L})\end{array}$ & $\begin{array}{c}\text { Cadmium } \\
(\mathrm{mg} / \mathrm{L})\end{array}$ & $\begin{array}{c}\mathrm{DO} \\
(\mathrm{mg} / \mathrm{L})\end{array}$ & $\begin{array}{l}\text { Cond. } \\
(\mu \mathrm{S} / \mathrm{cm})\end{array}$ & $\begin{array}{c}\mathrm{pH} \\
\text { (S.U.) } \\
\end{array}$ & $\begin{array}{c}\text { Redox } \\
(\mathrm{mV})\end{array}$ & $\begin{array}{c}\text { Temp } \\
\left({ }^{\circ} \mathrm{C}\right)\end{array}$ & $\begin{array}{c}\text { Alkalinity } \\
(\mathrm{mg} / \mathrm{L} \text { as } \\
\left.\mathrm{CaCO}_{3}\right)\end{array}$ & $\begin{array}{c}\text { Hardness } \\
(\mathrm{mg} / \mathrm{L} \text { as } \\
\left.\mathrm{CaCO}_{3}\right)\end{array}$ \\
\hline Inflow & 0.1 & 6.1 & 0.1006 & 0.0200 & 0.000281 & nd & 7970 & nd & nd & nd & 432 & 1260 \\
\hline $\mathrm{A} 1^{\mathrm{a}}$ & $<0.03$ & 5.0 & 0.0548 & 0.0344 & 0.000061 & 4.65 & 7700 & 7.71 & -10 & 18.2 & 550 & 1080 \\
\hline $\mathrm{A} 2$ & 0.8 & 3.6 & 0.2437 & 0.2638 & 0.000035 & 4.2 & 6780 & 7.46 & -415 & 17.5 & 490 & 1260 \\
\hline A3 & $<0.03$ & 1.7 & 0.368 & 0.2174 & 0.000101 & 6.91 & 6730 & 7.47 & -385 & 17.5 & 396 & 1440 \\
\hline A4 & 0.1 & 0.54 & 0.2032 & 0.0372 & $<0.000025$ & 4.38 & 6870 & 7.39 & 106 & 17.7 & 416 & 1440 \\
\hline B1 & $<0.03$ & 5.1 & 0.0186 & 0.0050 & 0.000036 & 6.13 & 7970 & 7.97 & 59 & 18.7 & 422 & 1440 \\
\hline B2 & $<0.03$ & 3.7 & 0.0203 & 0.0071 & 0.000027 & 7.08 & 7970 & 7.82 & -225 & 18.3 & 418 & 1260 \\
\hline B3 & $<0.03$ & 1.1 & 0.0245 & 0.0115 & 0.000025 & 3.7 & 7770 & 7.68 & -243 & 17.7 & 422 & 1260 \\
\hline B4 & $<0.03$ & 0.43 & 0.0138 & 0.0028 & $<0.000025$ & 4.39 & 7670 & 7.58 & 130 & 17.6 & 402 & 1440 \\
\hline $\mathrm{C} 1$ & $<0.03$ & 5.2 & 0.0392 & 0.0196 & 0.00014 & 7.46 & 7830 & 7.61 & 111 & 19.8 & 430 & 1260 \\
\hline $\mathrm{C} 2$ & $<0.03$ & 3.2 & 0.0388 & 2.830 & 0.00007 & 8.9 & 7400 & 7.49 & -346 & 19 & 450 & 1440 \\
\hline $\mathrm{C} 3$ & $<0.03$ & 1.9 & 0.0111 & 0.0242 & 0.000052 & 2.9 & 6560 & 7.65 & -216 & 18.1 & 362 & 1440 \\
\hline $\mathrm{C} 4$ & $<0.03$ & 0.31 & 0.0093 & 0.0146 & 0.000027 & 2.8 & 6050 & 7.22 & 80 & 18.1 & 340 & 900 \\
\hline D1 & $<0.03$ & 5.2 & 0.0444 & 0.0144 & 0.000036 & 2.56 & 7400 & 7.83 & 89 & 18.3 & 368 & 1260 \\
\hline D2 & $<0.03$ & 2.6 & 0.0260 & 0.0209 & 0.000088 & 4.27 & 6010 & 7.79 & -170 & 18 & 274 & 1080 \\
\hline D3 & $<0.03$ & 1.1 & 0.0337 & 0.0892 & $<0.000025$ & 1.4 & 7010 & 7.77 & -213 & 17.3 & 212 & 900 \\
\hline D4 & $<0.03$ & 0.24 & 0.0162 & 0.0541 & $<0.000025$ & 3.2 & 5680 & 7.48 & 143 & 17.5 & 216 & 1080 \\
\hline
\end{tabular}

nd- not determined

$\mathrm{Se}$ bdl $(0.0002 \mathrm{mg} / \mathrm{L})$.

${ }^{a}$ Sampling location format is series (A), cell (1), etc., (Figure 1). 
Table 71. COC concentrations and explanatory parameters for Sampling Period $3(10 / 4 / 12)$

\begin{tabular}{|c|c|c|c|c|c|c|c|c|c|}
\hline \multirow[b]{2}{*}{$\begin{array}{l}\text { Sampling } \\
\text { Location }\end{array}$} & \multicolumn{2}{|c|}{ Performance Parameters } & \multicolumn{7}{|c|}{ Explanatory Parameters } \\
\hline & $\begin{array}{c}\text { Ammonia } \\
(\mathrm{mg} / \mathrm{L})\end{array}$ & $\begin{array}{c}\text { Barium } \\
(\mathrm{mg} / \mathrm{L})\end{array}$ & $\begin{array}{c}\mathrm{DO} \\
(\mathrm{mg} / \mathrm{L})\end{array}$ & $\begin{array}{l}\text { Cond. } \\
(\mu \mathrm{S} / \mathrm{cm})\end{array}$ & $\begin{array}{c}\mathrm{pH} \\
(\mathrm{S} . \mathrm{U} .) \\
\end{array}$ & $\begin{array}{c}\text { Redox } \\
(\mathrm{mV})\end{array}$ & $\begin{array}{c}\text { Temp } \\
\left({ }^{\circ} \mathrm{C}\right)\end{array}$ & $\begin{array}{c}\text { Alkalinity } \\
(\mathrm{mg} / \mathrm{L} \text { as } \\
\left.\mathrm{CaCO}_{3}\right) \\
\end{array}$ & $\begin{array}{c}\text { Hardness } \\
(\mathrm{mg} / \mathrm{L} \text { as } \\
\mathrm{CaCO}_{3} \text { ) }\end{array}$ \\
\hline Inflow & 0.5 & 9.3 & nd & 9760 & nd & nd & nd & 456 & 2160 \\
\hline $\mathrm{A} 1^{\mathrm{a}}$ & 0.1 & 7.9 & 4.2 & 9650 & 7.71 & -22 & 24.9 & 446 & 1800 \\
\hline A2 & 1.0 & 4.1 & 6.67 & 9520 & 7.73 & -221 & 25.2 & 428 & 1980 \\
\hline A3 & 0.9 & 1.6 & 6.29 & 9010 & 7.07 & -320 & 24.7 & 410 & 1980 \\
\hline A4 & 0.1 & 0.28 & 4.26 & 8420 & 7.24 & 122 & 25.6 & 394 & 1800 \\
\hline B1 & 0.1 & 7.8 & 4.25 & 9360 & 7.55 & 305 & 24.9 & 432 & 1800 \\
\hline $\mathrm{B} 2$ & 0.1 & 4.2 & 4.55 & 9520 & 7.6 & -227 & 25 & 422 & 1800 \\
\hline B3 & $<0.03$ & 1.9 & 4.66 & 9570 & 7.28 & -240 & 23.7 & 420 & 1800 \\
\hline B4 & $<0.03$ & 0.17 & 4.21 & 9520 & 7.18 & 120 & 24 & 446 & 1800 \\
\hline $\mathrm{C} 1$ & 0.1 & 7.4 & 3.06 & 9680 & 7.11 & 124 & 24.3 & 430 & 1800 \\
\hline $\mathrm{C} 2$ & $<0.03$ & 3.3 & 7.08 & 9700 & 7.39 & -350 & 23.7 & 440 & 1800 \\
\hline $\mathrm{C} 3$ & $<0.03$ & 1.9 & 6.81 & 9660 & 7.67 & -212 & 24.1 & 462 & 1980 \\
\hline $\mathrm{C} 4$ & $<0.03$ & 0.82 & 5.11 & 9690 & 7.77 & 90 & 24.5 & 450 & 1800 \\
\hline D1 & 0.1 & 7.5 & 4.87 & 9580 & 7.89 & 74 & 24.1 & 448 & 1980 \\
\hline D2 & $<0.03$ & 4.5 & 6.37 & 9690 & 7.68 & -154 & 22.7 & 448 & 1800 \\
\hline D3 & $<0.03$ & 1.4 & 3.2 & 9650 & 7.07 & -230 & 22.5 & 458 & 1800 \\
\hline D4 & $<0.03$ & 0.39 & 3.7 & 9760 & 7.47 & 168 & 23.9 & 442 & 1800 \\
\hline
\end{tabular}

nd- not determined

Cd bdl (0.000025 mg/L), Fe bdl (0.0062 mg/L), Mn bdl $(0.0014 \mathrm{mg} / \mathrm{L})$, Se bdl $(0.0002 \mathrm{mg} / \mathrm{L})$

a Sampling location format is series (A), cell (1), etc., (Figure 1). 
Table 72. COC concentrations and explanatory parameters for Sampling Period 4 (10/18/12)

\begin{tabular}{|c|c|c|c|c|c|c|c|c|c|c|c|}
\hline \multirow[b]{2}{*}{$\begin{array}{l}\text { Sampling } \\
\text { Location }\end{array}$} & \multicolumn{4}{|c|}{ Performance Parameters } & \multicolumn{7}{|c|}{ Explanatory Parameters } \\
\hline & $\begin{array}{c}\text { Ammonia } \\
(\mathrm{mg} / \mathrm{L})\end{array}$ & $\begin{array}{c}\text { Barium } \\
(\mathrm{mg} / \mathrm{L})\end{array}$ & $\begin{array}{c}\text { Iron } \\
(\mathrm{mg} / \mathrm{L}) \\
\end{array}$ & $\begin{array}{c}\text { Manganese } \\
(\mathrm{mg} / \mathrm{L})\end{array}$ & $\begin{array}{c}\mathrm{DO} \\
(\mathrm{mg} / \mathrm{L}) \\
\end{array}$ & $\begin{array}{l}\text { Cond. } \\
(\mu \mathrm{S} / \mathrm{cm})\end{array}$ & $\begin{array}{c}\mathrm{pH} \\
\text { (S.U.) } \\
\end{array}$ & $\begin{array}{c}\text { Redox } \\
(\mathrm{mV})\end{array}$ & $\begin{array}{c}\text { Temp } \\
\left({ }^{\circ} \mathrm{C}\right)\end{array}$ & $\begin{array}{c}\text { Alkalinity } \\
(\mathrm{mg} / \mathrm{L} \text { as } \\
\left.\mathrm{CaCO}_{3}\right)\end{array}$ & $\begin{array}{c}\text { Hardness } \\
\text { (mg/L as } \\
\mathrm{CaCO}_{3} \text { ) }\end{array}$ \\
\hline Inflow & 3.3 & 17.0 & 2.44 & 0.169 & nd & 18300 & nd & nd & nd & 430 & 6660 \\
\hline $\mathrm{A} 1^{\mathrm{a}}$ & 2.6 & 13.0 & 1.32 & 0.104 & 3.85 & 24100 & 6.96 & 189 & 26.8 & 380 & 5400 \\
\hline $\mathrm{A} 2$ & 2.4 & 7.4 & 0.389 & $<0.0014$ & 7.35 & 19200 & 7.24 & -347 & 27 & 318 & 5220 \\
\hline A3 & 2.4 & 3.7 & 0.247 & $<0.0014$ & 7.01 & 16100 & 7.23 & -331 & 27 & 264 & 5040 \\
\hline A4 & 1.4 & 1.2 & 0.088 & $<0.0014$ & 6.71 & 17070 & 7.18 & 181 & 27.2 & 280 & 6120 \\
\hline B1 & 3.2 & 11.3 & 1.399 & 0.554 & 3.8 & 17900 & 6.41 & 211 & 26.8 & 400 & 5940 \\
\hline B2 & 2.5 & 6.7 & 0.324 & 0.113 & 5.3 & 17050 & 6.77 & -228 & 23.7 & 312 & 5580 \\
\hline B3 & 1.8 & 2.9 & 0.091 & $<0.0014$ & 4.36 & 18100 & 7.41 & -238 & 23.2 & 248 & 4500 \\
\hline B4 & 1.3 & 0.91 & 0.016 & $<0.0014$ & 3.46 & 17950 & 7.18 & 155 & 24 & 254 & 4860 \\
\hline $\mathrm{C} 1$ & 3 & 15.9 & 1.107 & 0.341 & 2.4 & 23100 & 7.11 & 108 & 24.3 & 398 & 6300 \\
\hline $\mathrm{C} 2$ & 2.3 & 10.9 & 0.117 & 0.051 & 2 & 24300 & 7.39 & -394 & 23.7 & 326 & 5220 \\
\hline $\mathrm{C} 3$ & 1.3 & 5.7 & 0.033 & $<0.0014$ & 3.65 & 22300 & 7.67 & -216 & 24.1 & 236 & 5400 \\
\hline $\mathrm{C} 4$ & 0.9 & 0.59 & 0.028 & $<0.0014$ & 2.71 & 18800 & 7.77 & 172 & 24.5 & 186 & 3240 \\
\hline D1 & 3.2 & 14.6 & 2.29 & 0.21 & 4.8 & 19060 & 7.89 & 98 & 24.1 & 400 & 5400 \\
\hline D2 & 3 & 10.7 & 0.351 & 0.006 & 5.4 & 17530 & 7.68 & -174 & 22.7 & 344 & 5220 \\
\hline D3 & 1.8 & 6.6 & 0.074 & $<0.0014$ & 3.28 & 18500 & 7.07 & -209 & 22.5 & 258 & 3960 \\
\hline D4 & 1.3 & 0.42 & 0.017 & $<0.0014$ & 3.69 & 17100 & 7.47 & 163 & 23.9 & 198 & 2880 \\
\hline
\end{tabular}

nd- not determined
$\mathrm{Cd}$ bdl $(0.000025 \mathrm{mg} / \mathrm{L}), \mathrm{Se}$ bdl $(0.0002 \mathrm{mg} / \mathrm{L})$

${ }^{a}$ Sampling location format is series (A), cell (1), etc., (Figure 1). 
Table 73. COC concentrations and explanatory parameters for Sampling Period 5 (11/1/12)

\begin{tabular}{|c|c|c|c|c|c|c|c|c|c|c|}
\hline \multirow[b]{2}{*}{$\begin{array}{l}\text { Sampling } \\
\text { Location }\end{array}$} & \multicolumn{3}{|c|}{ Performance Parameters } & \multicolumn{7}{|c|}{ Explanatory Parameters } \\
\hline & $\begin{array}{c}\text { Ammonia } \\
(\mathrm{mg} / \mathrm{L})\end{array}$ & $\begin{array}{c}\text { Barium } \\
(\mathrm{mg} / \mathrm{L})\end{array}$ & $\begin{array}{c}\text { Iron } \\
(\mathrm{mg} / \mathrm{L}) \\
\end{array}$ & $\begin{array}{c}\mathrm{DO} \\
(\mathrm{mg} / \mathrm{L}) \\
\end{array}$ & $\begin{array}{l}\text { Cond. } \\
(\mu \mathrm{S} / \mathrm{cm})\end{array}$ & $\begin{array}{c}\mathrm{pH} \\
\text { (S.U.) } \\
\end{array}$ & $\begin{array}{c}\text { Redox } \\
(\mathrm{mV})\end{array}$ & $\begin{array}{c}\text { Temp } \\
\left({ }^{\circ} \mathrm{C}\right)\end{array}$ & $\begin{array}{c}\text { Alkalinity } \\
(\mathrm{mg} / \mathrm{L} \text { as } \\
\left.\mathrm{CaCO}_{3}\right)\end{array}$ & $\begin{array}{c}\text { Hardness } \\
(\mathrm{mg} / \mathrm{L} \text { as } \\
\left.\mathrm{CaCO}_{3}\right)\end{array}$ \\
\hline Inflow & 2.3 & 13.2 & 1.48 & nd & 9120 & nd & nd & nd & 102 & 2070 \\
\hline $\mathrm{A} 1^{\mathrm{a}}$ & 1.9 & 11.6 & 0.43 & 7.73 & 11650 & 8.14 & 157 & 16.1 & 516 & 1440 \\
\hline $\mathrm{A} 2$ & 1.2 & 8.1 & 0.269 & 11.4 & 10360 & 8.28 & -319 & 15.3 & 506 & 2700 \\
\hline A3 & 0.8 & 4.7 & 0.115 & 13 & 10510 & 7.88 & -204 & 14.3 & 498 & 2700 \\
\hline A4 & 0.1 & 1.4 & 0.047 & 14.3 & 11200 & 8.02 & 210 & 14.5 & 480 & 2340 \\
\hline B1 & 2.2 & 10.3 & 0.907 & 2.06 & 12940 & 8.25 & 226 & 17.3 & 516 & 2160 \\
\hline B2 & 1.9 & 7.9 & 0.167 & 4.9 & 10380 & 7.76 & -219 & 13.1 & 508 & 2340 \\
\hline B3 & 1.8 & 5.7 & 0.109 & 10.2 & 10170 & 8.01 & -219 & 12.4 & 504 & 1980 \\
\hline B4 & 1 & 1.1 & 0.02 & 5.91 & 11520 & 7.84 & 162 & 13.9 & 490 & 1800 \\
\hline $\mathrm{C} 1$ & 1.5 & 11.6 & 0.98 & 5.6 & 12600 & 6.54 & 329 & 15.9 & 538 & 1980 \\
\hline $\mathrm{C} 2$ & 1.5 & 7.7 & 0.519 & 3.23 & 12440 & 6.82 & -268 & 16.8 & 516 & 1980 \\
\hline $\mathrm{C} 3$ & 1.9 & 3.1 & 0.496 & 4.1 & 13270 & 7.02 & -195 & 14.9 & 520 & 2520 \\
\hline $\mathrm{C} 4$ & 0.9 & 0.9 & 0.331 & 2.9 & 12010 & 6.5 & 242 & 15.9 & 490 & 2340 \\
\hline D1 & 2 & 9.9 & 0.476 & 6.64 & 10510 & 7.89 & 161 & 15.9 & 520 & 1800 \\
\hline D2 & 1.9 & 6.2 & 0.248 & 4.1 & 11040 & 7.68 & -196 & 16.8 & 540 & 1980 \\
\hline D3 & 1.5 & 2.6 & 0.14 & 2.33 & 11450 & 7.07 & -154 & 14.9 & 510 & 1980 \\
\hline D4 & 0.9 & 0.61 & 0.104 & 3.35 & 11170 & 7.47 & 172 & 15.9 & 504 & 1800 \\
\hline
\end{tabular}

nd- not determined
$\mathrm{Cd}$ bdl $(0.000025 \mathrm{mg} / \mathrm{L}), \mathrm{Mn}$ bdl $(0.0014 \mathrm{mg} / \mathrm{L}), \mathrm{Se}$ bdl $(0.0002 \mathrm{mg} / \mathrm{L})$.

a Sampling location format is series (A), cell (1), etc., (Figure 1). 
Table 74. COC concentrations and explanatory parameters for Sampling Period $6(11 / 15 / 12)$

\begin{tabular}{|c|c|c|c|c|c|c|c|c|c|c|}
\hline \multirow[b]{2}{*}{$\begin{array}{l}\text { Sampling } \\
\text { Location }\end{array}$} & \multicolumn{3}{|c|}{ Performance Parameters } & \multicolumn{7}{|c|}{ Explanatory Parameters } \\
\hline & $\begin{array}{c}\text { Ammonia } \\
(\mathrm{mg} / \mathrm{L})\end{array}$ & $\begin{array}{l}\text { Barium } \\
(\mathrm{mg} / \mathrm{L})\end{array}$ & $\begin{array}{l}\text { Iron } \\
(\mathrm{mg} / \mathrm{L})\end{array}$ & $\begin{array}{c}\mathrm{DO} \\
(\mathrm{mg} / \mathrm{L})\end{array}$ & $\begin{array}{l}\text { Cond. } \\
(\mu \mathrm{S} / \mathrm{cm})\end{array}$ & $\begin{array}{c}\mathrm{pH} \\
\text { (S.U.) }\end{array}$ & $\begin{array}{c}\text { Redox } \\
(\mathrm{mV})\end{array}$ & $\begin{array}{c}\text { Temp } \\
\left({ }^{\circ} \mathrm{C}\right)\end{array}$ & $\begin{array}{c}\text { Alkalinity } \\
(\mathrm{mg} / \mathrm{L} \text { as } \\
\left.\mathrm{CaCO}_{3}\right)\end{array}$ & $\begin{array}{l}\text { Hardness } \\
(\mathrm{mg} / \mathrm{L} \text { as } \\
\left.\mathrm{CaCO}_{3}\right) \\
\end{array}$ \\
\hline Inflow & 2.5 & 28.6 & 1.524 & nd & 13920 & nd & nd & nd & 470 & 3860 \\
\hline $\mathrm{A} 1^{\mathrm{a}}$ & 1.9 & 28.3 & 0.38 & 11.19 & 18410 & 7.79 & 197 & 20.4 & 464 & 3780 \\
\hline $\mathrm{A} 2$ & 1.3 & 27.3 & 0.38 & 11.4 & 14460 & 7.98 & -300 & 18.9 & 468 & 3420 \\
\hline $\mathrm{A} 3$ & 1.2 & 29.7 & 0.589 & 13 & 14550 & 8.43 & -189 & 18.8 & 430 & 3960 \\
\hline A4 & 1.1 & 28.8 & 0.119 & 12.2 & 14420 & 8.31 & 257 & 18.1 & 402 & 3780 \\
\hline B1 & 1.8 & 29.4 & 1.706 & 4.5 & 18600 & 7.19 & 252 & 19.5 & 472 & 3600 \\
\hline $\mathrm{B} 2$ & 1.7 & 27.4 & 0.272 & 10.6 & 14500 & 8.25 & -215 & 18 & 470 & 3420 \\
\hline B3 & 1.6 & 29.7 & 0.172 & 6.75 & 16010 & 7.55 & -218 & 17.2 & 452 & 3420 \\
\hline B4 & 1.2 & 29.6 & 0.07 & 8.37 & 14830 & 7.56 & 181 & 17.3 & 454 & 3960 \\
\hline $\mathrm{C} 1$ & 1.9 & 30.5 & 0.611 & 9.87 & 18290 & 7.09 & 259 & 19.5 & 486 & 4320 \\
\hline $\mathrm{C} 2$ & 1.6 & 28.2 & 0.418 & 10.1 & 15970 & 7.27 & -244 & 19.5 & 470 & 5040 \\
\hline $\mathrm{C} 3$ & 1.4 & 26.5 & 0.386 & 10.07 & 15800 & 6.68 & -186 & 19.3 & 454 & 3600 \\
\hline $\mathrm{C} 4$ & 1.2 & 25.6 & 0.123 & 9.8 & 14540 & 8.27 & 253 & 18.8 & 460 & 4680 \\
\hline D1 & 1.8 & 26.9 & 1.742 & 6.6 & 18500 & 7.07 & 168 & 19.3 & 470 & 4140 \\
\hline D2 & 1.7 & 26.2 & 0352 & 10.8 & 17020 & 7.03 & -100 & 18.4 & 460 & 3780 \\
\hline D3 & 1.4 & 26.1 & 0.255 & 2.53 & 14870 & 6.71 & -25 & 17.7 & 468 & 3780 \\
\hline D4 & 1.2 & 25.0 & 0.144 & 12.7 & 14430 & 8.41 & 184 & 18.4 & 446 & 3420 \\
\hline
\end{tabular}

nd- not determined
Cd bdl $(0.000025 \mathrm{mg} / \mathrm{L}), \mathrm{Mn}$ bdl $(0.0014 \mathrm{mg} / \mathrm{L})$, and Se bdl $(0.0002 \mathrm{mg} / \mathrm{L})$.

${ }^{a}$ Sampling location format is series (A), cell (1), etc., (Figure 1). 
Table 75. COC concentrations and explanatory parameters for Sampling Period 7 (11/29/12)

\begin{tabular}{|c|c|c|c|c|c|c|c|c|c|c|}
\hline \multirow[b]{2}{*}{$\begin{array}{l}\text { Sampling } \\
\text { Location }\end{array}$} & \multicolumn{3}{|c|}{ Performance Parameters } & \multicolumn{7}{|c|}{ Explanatory Parameters } \\
\hline & $\begin{array}{c}\text { Ammonia } \\
(\mathrm{mg} / \mathrm{L})\end{array}$ & $\begin{array}{c}\text { Barium } \\
(\mathrm{mg} / \mathrm{L})\end{array}$ & $\begin{array}{c}\text { Iron } \\
(\mathrm{mg} / \mathrm{L})\end{array}$ & $\begin{array}{c}\mathrm{DO} \\
(\mathrm{mg} / \mathrm{L}) \\
\end{array}$ & $\begin{array}{l}\text { Cond. } \\
(\mu \mathrm{S} / \mathrm{cm})\end{array}$ & $\begin{array}{c}\mathrm{pH} \\
\text { (S.U.) }\end{array}$ & $\begin{array}{c}\text { Redox } \\
(\mathrm{mV})\end{array}$ & $\begin{array}{l}\text { Temp } \\
\left({ }^{\circ} \mathrm{C}\right)\end{array}$ & $\begin{array}{c}\text { Alkalinity } \\
(\mathrm{mg} / \mathrm{L} \text { as } \\
\left.\mathrm{CaCO}_{3}\right)\end{array}$ & $\begin{array}{l}\text { Hardness } \\
(\mathrm{mg} / \mathrm{L} \text { as } \\
\left.\mathrm{CaCO}_{3}\right)\end{array}$ \\
\hline Inflow & 2.7 & 12.6 & 0.262 & nd & 9670 & nd & nd & nd & 456 & 2160 \\
\hline $\mathrm{A} 1^{\mathrm{a}}$ & 1.8 & 13.0 & 0.132 & 7.73 & 10760 & 7.98 & 202 & 14.7 & 446 & 1800 \\
\hline $\mathrm{A} 2$ & 1.5 & 13.2 & 0.156 & 11.4 & 10400 & 7.8 & -229 & 15.4 & 428 & 1980 \\
\hline A3 & 1.2 & 14.5 & 0.126 & 13 & 10830 & 7.98 & -200 & 13.4 & 410 & 1980 \\
\hline A4 & 0.8 & 14.6 & 0.109 & 12.2 & 10900 & 7.95 & 342 & 12 & 394 & 1800 \\
\hline B1 & 1.8 & 13.5 & 0.179 & 4.5 & 10190 & 6.91 & 257 & 14.2 & 432 & 1950 \\
\hline B2 & 1.6 & 13.7 & 0.118 & 10.6 & 10560 & 7.4 & -220 & 12.3 & 422 & 1890 \\
\hline B3 & 1.3 & 14.2 & 0.088 & 10.2 & 10800 & 6.82 & -227 & 12.8 & 420 & 1800 \\
\hline B4 & 0.9 & 13.9 & 0.055 & 5.91 & 12800 & 7.21 & 172 & 11.6 & 446 & 1800 \\
\hline $\mathrm{C} 1$ & 1.9 & 14.2 & 0.232 & 5.6 & 14300 & 6.54 & 262 & 14.8 & 430 & 1800 \\
\hline $\mathrm{C} 2$ & 1.5 & 14.3 & 0.178 & 10.1 & 13080 & 6.78 & -243 & 15.3 & 440 & 1800 \\
\hline $\mathrm{C} 3$ & 1.4 & 14.2 & 0.137 & 10.07 & 10100 & 6.84 & -205 & 14.1 & 462 & 1980 \\
\hline $\mathrm{C} 4$ & 0.8 & 15.5 & 0.089 & 9.8 & 10680 & 7.56 & 262 & 12.1 & 450 & 1800 \\
\hline D1 & 1.5 & 14.2 & 0.254 & 6.6 & 10600 & 6.73 & 245 & 13.5 & 448 & 1980 \\
\hline D2 & 1.2 & 12.9 & 0.042 & 10.8 & 13300 & 7.05 & -197 & 13.2 & 448 & 1800 \\
\hline D3 & 1.1 & 14.1 & 0.023 & 6.6 & 12400 & 7.24 & 44 & 12.5 & 458 & 1800 \\
\hline D4 & 0.8 & 14.0 & 0.013 & 9.8 & 10260 & 7.31 & 139 & 12.9 & 442 & 1800 \\
\hline
\end{tabular}

nd- not determined

${ }^{a}$ Sampling location format is series (A), cell (1), etc., (Figure 1). 
Table 76. COC concentrations and explanatory parameters for Sampling Period 8 (12/13/12)

\begin{tabular}{|c|c|c|c|c|c|c|c|c|c|c|c|}
\hline \multirow[b]{2}{*}{$\begin{array}{l}\text { Sampling } \\
\text { Location }\end{array}$} & \multicolumn{4}{|c|}{ Performance Parameters } & \multicolumn{7}{|c|}{ Explanatory Parameters } \\
\hline & $\begin{array}{c}\text { Ammonia } \\
(\mathrm{mg} / \mathrm{L})\end{array}$ & $\begin{array}{l}\text { Barium } \\
(\mathrm{mg} / \mathrm{L})\end{array}$ & $\begin{array}{c}\text { Iron } \\
(\mathrm{mg} / \mathrm{L})\end{array}$ & $\begin{array}{c}\text { Manganese } \\
(\mathrm{mg} / \mathrm{L})\end{array}$ & $\begin{array}{c}\mathrm{DO} \\
(\mathrm{mg} / \mathrm{L}) \\
\end{array}$ & $\begin{array}{l}\text { Cond. } \\
(\mu \mathrm{S} / \mathrm{cm})\end{array}$ & $\begin{array}{c}\mathrm{pH} \\
(\mathrm{S} . \mathrm{U} .) \\
\end{array}$ & $\begin{array}{c}\text { Redox } \\
(\mathrm{mV}) \\
\end{array}$ & $\begin{array}{c}\text { Temp } \\
\left({ }^{\circ} \mathrm{C}\right)\end{array}$ & $\begin{array}{c}\text { Alkalinity } \\
(\mathrm{mg} / \mathrm{L} \text { as } \\
\left.\mathrm{CaCO}_{3}\right)\end{array}$ & $\begin{array}{c}\text { Hardness } \\
(\mathrm{mg} / \mathrm{L} \text { as } \\
\left.\mathrm{CaCO}_{3}\right)\end{array}$ \\
\hline Inflow & 2.9 & 7.5 & 0.7842 & 0.0299 & nd & 6800 & nd & nd & nd & 490 & 2160 \\
\hline $\mathrm{A} 1^{\mathrm{a}}$ & 1.2 & 7.4 & 0.1299 & 0.0175 & 6.3 & 7380 & 7.98 & 181 & 13.7 & 452 & 1440 \\
\hline $\mathrm{A} 2$ & 1.1 & 5.8 & 0.1933 & 0.0239 & 8.1 & 7280 & 7.37 & -320 & 13.4 & 476 & 1440 \\
\hline A3 & 0.8 & 3.8 & 0.2512 & 0.0275 & 8.9 & 7430 & 7.98 & -299 & 12.4 & 430 & 2160 \\
\hline A4 & 0.4 & 1.8 & 0.2168 & $<0.0014$ & 10.4 & 7470 & 7.01 & 257 & 12 & 462 & 1800 \\
\hline $\mathrm{B} 1$ & 1.2 & 7.4 & 0.1696 & 0.0114 & 7.1 & 7030 & 6.91 & 252 & 12.2 & 472 & 1440 \\
\hline $\mathrm{B} 2$ & 1.1 & 5.5 & 0.1068 & 0.0099 & 9.8 & 7300 & 7.4 & -215 & 11.3 & 470 & 1980 \\
\hline B3 & 0.8 & 3.4 & 0.1314 & 0.0035 & 10.2 & 7350 & 6.82 & -218 & 12.8 & 452 & 1080 \\
\hline B4 & 0.5 & 1.9 & 0.0990 & $<0.0014$ & 8.6 & 7390 & 7.04 & 180 & 11.6 & 484 & 1080 \\
\hline $\mathrm{C} 1$ & 1.6 & 7.7 & 0.2120 & 0.0237 & 6.5 & 9300 & 6.54 & 260 & 13.8 & 486 & 2160 \\
\hline $\mathrm{C} 2$ & 1.2 & 6.7 & 0.2352 & 0.0202 & 10.4 & 10000 & 6.74 & -272 & 13.3 & 470 & 2520 \\
\hline $\mathrm{C} 3$ & 0.6 & 4.8 & 0.2857 & 0.0171 & 9.5 & 7130 & 6.84 & -183 & 12.1 & 454 & 1800 \\
\hline $\mathrm{C} 4$ & 0.2 & 2.9 & 0.1481 & $<0.0014$ & 9.9 & 7200 & 7.56 & 213 & 11.1 & 514 & 2340 \\
\hline D1 & 1.2 & 7.4 & 0.1149 & 0.0189 & 8.9 & 9210 & 6.42 & 171 & 12.5 & 470 & 1800 \\
\hline D2 & 1 & 7.1 & 0.3626 & 0.0196 & 10.7 & 11900 & 7.05 & -200 & 12.2 & 460 & 1980 \\
\hline D3 & 0.9 & 5.8 & 0.0566 & $<0.0014$ & 8.6 & 7240 & 6.24 & -47 & 11.5 & 468 & 2340 \\
\hline D4 & 0.3 & 3.1 & 0.0554 & $<0.0014$ & 9.2 & 7500 & 7.31 & 174 & 11.9 & 506 & 1620 \\
\hline
\end{tabular}

nd- not determined

Cd bdl $(0.000025 \mathrm{mg} / \mathrm{L})$, Se bdl $(0.0002 \mathrm{mg} / \mathrm{L})$

a Sampling location format is series (A), cell (1), etc., (Figure 1). 
Table 77. COC concentrations and explanatory parameters for Sampling Period 9 (1/10/13)

\begin{tabular}{|c|c|c|c|c|c|c|c|c|c|c|c|}
\hline \multirow[b]{2}{*}{$\begin{array}{l}\text { Sampling } \\
\text { Location }\end{array}$} & \multicolumn{4}{|c|}{ Performance Parameters } & \multicolumn{7}{|c|}{ Explanatory Parameters } \\
\hline & $\begin{array}{c}\text { Ammonia } \\
(\mathrm{mg} / \mathrm{L})\end{array}$ & $\begin{array}{l}\text { Barium } \\
(\mathrm{mg} / \mathrm{L})\end{array}$ & $\begin{array}{c}\text { Iron } \\
(\mathrm{mg} / \mathrm{L})\end{array}$ & $\begin{array}{c}\text { Manganese } \\
(\mathrm{mg} / \mathrm{L})\end{array}$ & $\begin{array}{c}\mathrm{DO} \\
(\mathrm{mg} / \mathrm{L})\end{array}$ & $\begin{array}{l}\text { Cond. } \\
(\mu \mathrm{S} / \mathrm{cm})\end{array}$ & $\begin{array}{c}\mathrm{pH} \\
(\mathrm{S} . \mathrm{U} .) \\
\end{array}$ & $\begin{array}{c}\text { Redox } \\
(\mathrm{mV})\end{array}$ & $\begin{array}{l}\text { Temp } \\
\left({ }^{\circ} \mathrm{C}\right)\end{array}$ & $\begin{array}{c}\text { Alkalinity } \\
(\mathrm{mg} / \mathrm{L} \text { as } \\
\left.\mathrm{CaCO}_{3}\right)\end{array}$ & $\begin{array}{c}\text { Hardness } \\
(\mathrm{mg} / \mathrm{L} \text { as } \\
\left.\mathrm{CaCO}_{3}\right)\end{array}$ \\
\hline Inflow & 1.6 & 7.4 & 0.1316 & 0.0341 & nd & 8100 & nd & nd & nd & 490 & 1080 \\
\hline $\mathrm{A} 1^{\mathrm{a}}$ & 1.1 & 7.1 & 0.1171 & 0.1237 & 6.7 & 9380 & 6.86 & 181 & 13 & 452 & 2160 \\
\hline $\mathrm{A} 2$ & 0.8 & 5.7 & 0.1043 & 0.1023 & 9.4 & 10000 & 7.37 & -396 & 12.2 & 476 & 2520 \\
\hline A3 & 0.5 & 3.9 & 0.0929 & 0.0329 & 10 & 9500 & 7.56 & -379 & 12 & 430 & 1800 \\
\hline A4 & 0.1 & 1.1 & 0.0912 & 0.0274 & 10.2 & 8870 & 8.1 & 277 & 11.8 & 462 & 2340 \\
\hline B1 & 1.4 & 6.7 & 0.468 & 0.421 & 6.65 & 8900 & 7.01 & 255 & 11.7 & 472 & 1800 \\
\hline B2 & 1 & 5.2 & 0.1082 & 0.1423 & 10.6 & 11600 & 7.16 & -226 & 11.6 & 470 & 1980 \\
\hline B3 & 0.7 & 3.2 & 0.0717 & 0.0513 & 10.2 & 9020 & 7.04 & -238 & 11.2 & 452 & 2340 \\
\hline B4 & 0.3 & 1.5 & 0.0444 & 0.0079 & 7.91 & 9010 & 7.6 & 170 & 11.6 & 484 & 1620 \\
\hline $\mathrm{C} 1$ & 1.3 & 7.3 & 0.3882 & 0.0861 & 6.3 & 13900 & 6.77 & 255 & 11.7 & 456 & 1440 \\
\hline $\mathrm{C} 2$ & 0.9 & 5.5 & 0.1809 & 0.1643 & 10.1 & 12800 & 6.74 & -308 & 11.4 & 446 & 1440 \\
\hline $\mathrm{C} 3$ & 0.5 & 4.3 & 0.1543 & 0.0471 & 9.9 & 9990 & 6.7 & -283 & 12 & 428 & 2160 \\
\hline $\mathrm{C} 4$ & 0.1 & 3.1 & 0.0675 & $<0.0014$ & 9.6 & 8850 & 7.99 & 220 & 11.5 & 410 & 1800 \\
\hline D1 & 1.1 & 7.3 & 0.1268 & 0.1094 & 9.3 & 12400 & 6.42 & 218 & 11.8 & 394 & 1440 \\
\hline D2 & 0.8 & 6.3 & 0.104 & 0.0643 & 10.8 & 10200 & 6.68 & -267 & 11.5 & 432 & 1980 \\
\hline D3 & 0.6 & 4.4 & 0.0561 & 0.0089 & 8.6 & 10190 & 6.7 & -81 & 11.8 & 422 & 1080 \\
\hline D4 & 0.2 & 3.4 & 0.0060 & 0.0111 & 9.9 & 9750 & 6.68 & 274 & 11.3 & 420 & 1080 \\
\hline
\end{tabular}

nd- not determined

${ }^{a}$ Sampling location format is series (A), cell (1), etc., (Figure 1). 
Table 78. COC concentrations and explanatory parameters for Sampling Period 10 (1/24/13)

\begin{tabular}{|c|c|c|c|c|c|c|c|c|c|c|c|}
\hline \multirow[b]{2}{*}{$\begin{array}{l}\text { Sampling } \\
\text { Location }\end{array}$} & \multicolumn{4}{|c|}{ Performance Parameters } & \multicolumn{7}{|c|}{ Explanatory Parameters } \\
\hline & $\begin{array}{c}\text { Ammonia } \\
(\mathrm{mg} / \mathrm{L})\end{array}$ & $\begin{array}{c}\text { Barium } \\
(\mathrm{mg} / \mathrm{L})\end{array}$ & $\begin{array}{c}\text { Iron } \\
(\mathrm{mg} / \mathrm{L})\end{array}$ & $\begin{array}{c}\text { Manganese } \\
(\mathrm{mg} / \mathrm{L})\end{array}$ & $\begin{array}{c}\mathrm{DO} \\
(\mathrm{mg} / \mathrm{L}) \\
\end{array}$ & $\begin{array}{l}\text { Cond. } \\
(\mu \mathrm{S} / \mathrm{cm})\end{array}$ & $\begin{array}{c}\mathrm{pH} \\
\text { (S.U.) } \\
\end{array}$ & $\begin{array}{c}\text { Redox } \\
(\mathrm{mV})\end{array}$ & $\begin{array}{c}\text { Temp } \\
\left({ }^{\circ} \mathrm{C}\right)\end{array}$ & $\begin{array}{c}\text { Alkalinity } \\
(\mathrm{mg} / \mathrm{L} \text { as } \\
\left.\mathrm{CaCO}_{3}\right)\end{array}$ & $\begin{array}{c}\text { Hardness } \\
(\mathrm{mg} / \mathrm{L} \text { as } \\
\left.\mathrm{CaCO}_{3}\right)\end{array}$ \\
\hline Inflow & 1.7 & 6.2 & 0.3554 & 0.0385 & nd & 7300 & nd & nd & nd & 460 & 2080 \\
\hline $\mathrm{A} 1^{\mathrm{a}}$ & 0.9 & 5.7 & 0.3114 & 0.0221 & 8.1 & 8200 & 6.9 & 202 & 14.6 & 442 & 2130 \\
\hline $\mathrm{A} 2$ & 0.7 & 4.5 & 0.3067 & 0.0575 & 11.2 & 7790 & 7.8 & -276 & 15.2 & 476 & 2420 \\
\hline A3 & 0.5 & 3.1 & 0.2255 & 0.0556 & 10.3 & 7910 & 7.6 & -242 & 14.7 & 419 & 2100 \\
\hline A4 & 0.2 & 2.4 & 0.1308 & 0.0377 & 8.6 & 7300 & 6.9 & 342 & 11.6 & 458 & 2140 \\
\hline B1 & 1.4 & 5.6 & 0.3577 & 0.0664 & 5.4 & 8900 & 7.59 & 256 & 15.1 & 490 & 1900 \\
\hline B2 & 1.1 & 4.6 & 0.2548 & 0.0221 & 6.48 & 8300 & 6.86 & -220 & 13.5 & 480 & 1980 \\
\hline B3 & 0.7 & 4.5 & 0.2093 & 0.0043 & 7.1 & 7800 & 6.7 & -238 & 11.9 & 452 & 2340 \\
\hline B4 & 0.2 & 2.7 & 0.0559 & $<0.0014$ & 6.41 & 8080 & 7.34 & 128 & 10.9 & 460 & 1820 \\
\hline $\mathrm{C} 1$ & 1.3 & 5.8 & 0.4600 & 0.0454 & 6.4 & 8070 & 6.6 & 262 & 14.6 & 398 & 1640 \\
\hline $\mathrm{C} 2$ & 0.9 & 4.8 & 0.3208 & 0.0364 & 6.01 & 7810 & 7.9 & -244 & 13.1 & 431 & 1540 \\
\hline $\mathrm{C} 3$ & 0.6 & 2.6 & 0.2463 & 0.0193 & 5.7 & 7780 & 6.86 & -186 & 12.4 & 428 & 1960 \\
\hline $\mathrm{C} 4$ & 0.2 & 1.4 & 0.1144 & 0.0335 & 7.13 & 7840 & 6.12 & 262 & 12 & 450 & 2000 \\
\hline D1 & 1.1 & 5.9 & 0.1846 & 0.0283 & 6.3 & 7730 & 6.96 & 245 & 11.5 & 394 & 1740 \\
\hline D2 & 0.8 & 3.7 & 0.1537 & 0.0329 & 6.12 & 7850 & 7.8 & -200 & 11.2 & 432 & 1880 \\
\hline D3 & 0.6 & 1.7 & 0.0701 & $<0.0014$ & 7.4 & 7890 & 6.63 & -47 & 12.1 & 439 & 1180 \\
\hline D4 & 0.3 & 1.6 & 0.0371 & $<0.0014$ & 8.7 & 8000 & 6.87 & 184 & 11.7 & 427 & 1000 \\
\hline
\end{tabular}

nd- not determined
Cd bdl $(0.000025 \mathrm{mg} / \mathrm{L})$, Se bdl $(0.0001 \mathrm{mg} / \mathrm{L})$.

${ }^{a}$ Sampling location format is series (A), cell (1), etc., (Figure 1). 
Table 79. COC concentrations and explanatory parameters for Sampling Period $11(2 / 7 / 13)$

\begin{tabular}{|c|c|c|c|c|c|c|c|c|c|c|}
\hline \multirow[b]{2}{*}{$\begin{array}{l}\text { Sampling } \\
\text { Location }\end{array}$} & \multicolumn{3}{|c|}{ Performance Parameters } & \multicolumn{7}{|c|}{ Explanatory Parameters } \\
\hline & $\begin{array}{c}\text { Ammonia } \\
(\mathrm{mg} / \mathrm{L})\end{array}$ & $\begin{array}{l}\text { Barium } \\
(\mathrm{mg} / \mathrm{L})\end{array}$ & $\begin{array}{c}\text { Iron } \\
(\mathrm{mg} / \mathrm{L})\end{array}$ & $\begin{array}{c}\mathrm{DO} \\
(\mathrm{mg} / \mathrm{L})\end{array}$ & $\begin{array}{l}\text { Cond. } \\
(\mu \mathrm{S} / \mathrm{cm})\end{array}$ & $\begin{array}{c}\mathrm{pH} \\
\text { (S.U.) }\end{array}$ & $\begin{array}{c}\text { Redox } \\
(\mathrm{mV})\end{array}$ & $\begin{array}{l}\text { Temp } \\
\left({ }^{\circ} \mathrm{C}\right)\end{array}$ & $\begin{array}{c}\text { Alkalinity } \\
(\mathrm{mg} / \mathrm{L} \text { as } \\
\left.\mathrm{CaCO}_{3}\right)\end{array}$ & $\begin{array}{l}\text { Hardness } \\
(\mathrm{mg} / \mathrm{L} \text { as } \\
\left.\mathrm{CaCO}_{3}\right)\end{array}$ \\
\hline Inflow & 1.5 & 5.4 & 0.5258 & nd & 6830 & nd & nd & nd & 514 & 1980 \\
\hline $\mathrm{A} 1^{\mathrm{a}}$ & 1.2 & 5.2 & 0.5082 & 9.6 & 7080 & 7.9 & 202 & 14.6 & 452 & 1260 \\
\hline $\mathrm{A} 2$ & 2.6 & 4.2 & 0.3580 & 13.2 & 7200 & 7.8 & -226 & 15.2 & 490 & 1260 \\
\hline A3 & 1.2 & 4.2 & 0.2145 & 12.3 & 7270 & 7.6 & -227 & 14.7 & 422 & 1980 \\
\hline A4 & 0.6 & 2.7 & 0.2035 & 9.6 & 7190 & 6.9 & 344 & 11.6 & 462 & 1620 \\
\hline B1 & 1.9 & 4.6 & 0.6010 & 4.4 & 7310 & 6.59 & 282 & 15.1 & 472 & 1260 \\
\hline B2 & 1.8 & 4.4 & 0.6834 & 5.48 & 7600 & 6.86 & -229 & 13.5 & 470 & 1800 \\
\hline B3 & 1.7 & 3.9 & 0.3252 & 6.94 & 7240 & 7.7 & -189 & 11.9 & 450 & 900 \\
\hline B4 & 1.3 & 2.7 & 0.2382 & 6.41 & 7400 & 7.34 & 120 & 10.9 & 484 & 900 \\
\hline $\mathrm{C} 1$ & 1.4 & 5.2 & 0.5442 & 6.4 & 8200 & 6.6 & 137 & 14.6 & 466 & 1980 \\
\hline $\mathrm{C} 2$ & 1.5 & 4.7 & 0.3066 & 6.78 & 8020 & 6.9 & -195 & 13.1 & 470 & 2340 \\
\hline $\mathrm{C} 3$ & 1.2 & 4.1 & 0.2327 & 5.7 & 7640 & 6.86 & -200 & 12.4 & 454 & 1620 \\
\hline $\mathrm{C} 4$ & 1 & 2.9 & 0.1610 & 8.13 & 7180 & 7.67 & 260 & 12 & 511 & 2160 \\
\hline D1 & 1.3 & 5.2 & 0.5377 & 6.3 & 8600 & 6.46 & 185 & 11.5 & 470 & 1620 \\
\hline D2 & 1.2 & 4.9 & 0.3413 & 6.12 & 7200 & 6.84 & -212 & 11.2 & 460 & 1800 \\
\hline D3 & 0.8 & 4.7 & 0.2671 & 7.4 & 7330 & 6.63 & -158 & 12.1 & 453 & 2160 \\
\hline D4 & 0.4 & 3.3 & 0.1473 & 7.7 & 7260 & 6.8 & 139 & 11.7 & 497 & 1440 \\
\hline
\end{tabular}


Table 80. COC concentrations and explanatory parameters for Sampling Period 12 (2/21/13)

\begin{tabular}{|c|c|c|c|c|c|c|c|c|c|c|}
\hline \multirow[b]{2}{*}{$\begin{array}{l}\text { Sampling } \\
\text { Location }\end{array}$} & \multicolumn{3}{|c|}{ Performance Parameters } & \multicolumn{7}{|c|}{ Explanatory Parameters } \\
\hline & $\begin{array}{c}\text { Ammonia } \\
(\mathrm{mg} / \mathrm{L})\end{array}$ & $\begin{array}{c}\text { Barium } \\
(\mathrm{mg} / \mathrm{L})\end{array}$ & $\begin{array}{c}\text { Iron } \\
(\mathrm{mg} / \mathrm{L})\end{array}$ & $\begin{array}{c}\mathrm{DO} \\
(\mathrm{mg} / \mathrm{L})\end{array}$ & $\begin{array}{l}\text { Cond. } \\
(\mu \mathrm{S} / \mathrm{cm})\end{array}$ & $\begin{array}{c}\mathrm{pH} \\
\text { (S.U.) } \\
\end{array}$ & $\begin{array}{c}\text { Redox } \\
(\mathrm{mV})\end{array}$ & $\begin{array}{l}\text { Temp } \\
\left({ }^{\circ} \mathrm{C}\right)\end{array}$ & $\begin{array}{c}\text { Alkalinity } \\
(\mathrm{mg} / \mathrm{L} \text { as } \\
\left.\mathrm{CaCO}_{3}\right)\end{array}$ & $\begin{array}{c}\text { Hardness } \\
(\mathrm{mg} / \mathrm{L} \text { as } \\
\left.\mathrm{CaCO}_{3}\right)\end{array}$ \\
\hline Inflow & 2 & 42.1 & 0.5589 & nd & 5980 & nd & nd & nd & 453 & 1990 \\
\hline $\mathrm{A} 1^{\mathrm{a}}$ & 1.5 & 34.6 & 0.4719 & 8.1 & 6120 & 7.6 & 251 & 12.5 & 441 & 1800 \\
\hline $\mathrm{A} 2$ & 0.9 & 8.9 & 0.4007 & 9.8 & 6310 & 7.77 & -230 & 13.6 & 429 & 1980 \\
\hline A3 & 0.8 & 5.7 & 0.4645 & 11.3 & 6470 & 7.58 & -211 & 12.8 & 410 & 1980 \\
\hline A4 & 1.2 & 2.4 & 0.3387 & 9.6 & 6200 & 7.91 & 341 & 10.2 & 394 & 1800 \\
\hline B1 & 2.5 & 38.9 & 0.692 & 6.1 & 6920 & 6.96 & 300 & 11.3 & 432 & 1800 \\
\hline B2 & 1.5 & 24.4 & 0.3675 & 7.48 & 7100 & 7.2 & -249 & 9.9 & 421 & 1800 \\
\hline B3 & 1.5 & 7.8 & 0.3333 & 8.71 & 6650 & 7.82 & -169 & 10.4 & 420 & 1800 \\
\hline B4 & 1.3 & 2.1 & 0.324 & 6.2 & 6810 & 7.1 & 220 & 10.7 & 446 & 1800 \\
\hline $\mathrm{C} 1$ & 2.3 & 40.9 & 0.391 & 7.4 & 6420 & 6.94 & 187 & 12.6 & 444 & 1800 \\
\hline $\mathrm{C} 2$ & 1.6 & 32.8 & 0.3843 & 7.58 & 6130 & 7.48 & -205 & 10.1 & 440 & 1800 \\
\hline $\mathrm{C} 3$ & 1.4 & 13.2 & 0.2953 & 6.1 & 6880 & 6.89 & -240 & 11.4 & 462 & 1980 \\
\hline $\mathrm{C} 4$ & 1.2 & 2.4 & 0.2439 & 10.3 & 7120 & 7.31 & 278 & 11.5 & 450 & 1800 \\
\hline D1 & 1.9 & 40.8 & 0.3583 & 8.2 & 6200 & 6.22 & 235 & 11.8 & 445 & 1980 \\
\hline D2 & 1.4 & 36.4 & 0.3474 & 7.75 & 6510 & 7.15 & -232 & 10.2 & 448 & 1800 \\
\hline D3 & 0.7 & 21.2 & 0.2851 & 7.4 & 6600 & 6.74 & -198 & 9.1 & 458 & 1800 \\
\hline D4 & 0.8 & 3.9 & 0.1852 & 6.93 & 6830 & 6.98 & 163 & 10.7 & 426 & 1800 \\
\hline
\end{tabular}


Table 81. COC concentrations and explanatory parameters for Sampling Period 13 (3/14/13)

\begin{tabular}{|c|c|c|c|c|c|c|c|c|c|c|c|}
\hline \multirow[b]{2}{*}{$\begin{array}{l}\text { Sampling } \\
\text { Location }\end{array}$} & \multicolumn{4}{|c|}{ Performance Parameters } & \multicolumn{7}{|c|}{ Explanatory Parameters } \\
\hline & $\begin{array}{c}\text { Ammonia } \\
(\mathrm{mg} / \mathrm{L})\end{array}$ & $\begin{array}{c}\text { Barium } \\
(\mathrm{mg} / \mathrm{L})\end{array}$ & $\begin{array}{c}\text { Iron } \\
(\mathrm{mg} / \mathrm{L})\end{array}$ & $\begin{array}{c}\text { Manganese } \\
(\mathrm{mg} / \mathrm{L})\end{array}$ & $\begin{array}{c}\mathrm{DO} \\
(\mathrm{mg} / \mathrm{L})\end{array}$ & $\begin{array}{l}\text { Cond. } \\
(\mu \mathrm{S} / \mathrm{cm})\end{array}$ & $\begin{array}{c}\mathrm{pH} \\
\text { (S.U.) } \\
\end{array}$ & $\begin{array}{c}\text { Redox } \\
(\mathrm{mV})\end{array}$ & $\begin{array}{c}\text { Temp } \\
\left({ }^{\circ} \mathrm{C}\right)\end{array}$ & $\begin{array}{c}\text { Alkalinity } \\
(\mathrm{mg} / \mathrm{L} \text { as } \\
\left.\mathrm{CaCO}_{3}\right)\end{array}$ & $\begin{array}{l}\text { Hardness } \\
(\mathrm{mg} / \mathrm{L} \text { as } \\
\left.\mathrm{CaCO}_{3}\right)\end{array}$ \\
\hline Inflow & 1.5 & 7.5 & 0.5386 & 0.0659 & nd & 7810 & nd & nd & nd & 508 & 1880 \\
\hline $\mathrm{A} 1^{\mathrm{a}}$ & 1.3 & 7.3 & 0.525 & 0.0864 & 8.6 & 8120 & 7.23 & 260 & 12.7 & 460 & 1260 \\
\hline $\mathrm{A} 2$ & 0.7 & 7.2 & 0.5714 & 0.0761 & 9.4 & 8440 & 8.21 & -211 & 11.6 & 490 & 1260 \\
\hline A3 & 0.2 & 6.1 & 0.5044 & 0.0634 & 10.3 & 7900 & 7.89 & -220 & 9.8 & 422 & 1980 \\
\hline A4 & 0.1 & 2.9 & 0.3061 & 0.0448 & 9.7 & 7630 & 7.7 & 321 & 10.2 & 461 & 1620 \\
\hline B1 & 1.6 & 6.9 & 0.4652 & 0.0771 & 7.2 & 7760 & 6.9 & 320 & 9.3 & 472 & 1260 \\
\hline $\mathrm{B} 2$ & 1.2 & 5.9 & 0.4336 & 0.0581 & 6.98 & 8030 & 7.29 & -219 & 9.9 & 470 & 1800 \\
\hline B3 & 0.9 & 4.6 & 0.416 & 0.0612 & 8.25 & 7860 & 7.35 & -178 & 10.5 & 438 & 900 \\
\hline B4 & 0.4 & 2.1 & 0.3229 & 0.0431 & 6.29 & 8290 & 7.27 & 223 & 9.1 & 484 & 900 \\
\hline $\mathrm{C} 1$ & 1.3 & 7.4 & 0.5251 & 0.0713 & 7.8 & 7860 & 6.99 & 198 & 11.6 & 466 & 1980 \\
\hline $\mathrm{C} 2$ & 1 & 6.6 & 0.4447 & 0.0702 & 8.78 & 7740 & 8 & -180 & 9.4 & 472 & 2340 \\
\hline $\mathrm{C} 3$ & 0.7 & 5.6 & 0.3785 & 0.0528 & 6.61 & 7700 & 7.12 & -232 & 10.4 & 449 & 1620 \\
\hline $\mathrm{C} 4$ & 0.2 & 3.1 & 0.2607 & 0.0382 & 9.64 & 8080 & 7.3 & 196 & 10.5 & 498 & 1860 \\
\hline D1 & 1.3 & 6.9 & 0.4122 & 0.0791 & 8.4 & 7530 & 6.67 & 220 & 9.8 & 470 & 1620 \\
\hline D2 & 0.8 & 6.5 & 0.4074 & 0.0671 & 7.65 & 7140 & 7.33 & -255 & 10.8 & 460 & 1800 \\
\hline D3 & 0.2 & 6.4 & 0.3828 & 0.0365 & 7.3 & 7890 & 6.8 & -209 & 10.1 & 453 & 1960 \\
\hline D4 & 0.1 & 3.9 & 0.2479 & 0.0279 & 5.9 & 7990 & 7.2 & 180 & 10 & 483 & 1640 \\
\hline
\end{tabular}

nd- not determined

${ }^{a}$ Sampling location format is series (A), cell (1), etc., (Figure 1). 
Table 82. COC concentrations and explanatory parameters for Sampling Period 14 (3/28/13)

\begin{tabular}{|c|c|c|c|c|c|c|c|c|c|c|c|}
\hline \multirow[b]{2}{*}{$\begin{array}{l}\text { Sampling } \\
\text { Location }\end{array}$} & \multicolumn{4}{|c|}{ Performance Parameters } & \multicolumn{7}{|c|}{ Explanatory Parameters } \\
\hline & $\begin{array}{c}\text { Ammonia } \\
(\mathrm{mg} / \mathrm{L})\end{array}$ & $\begin{array}{l}\text { Barium } \\
(\mathrm{mg} / \mathrm{L})\end{array}$ & $\begin{array}{c}\text { Iron } \\
(\mathrm{mg} / \mathrm{L})\end{array}$ & $\begin{array}{c}\text { Manganese } \\
(\mathrm{mg} / \mathrm{L})\end{array}$ & $\begin{array}{c}\mathrm{DO} \\
(\mathrm{mg} / \mathrm{L})\end{array}$ & $\begin{array}{l}\text { Cond. } \\
(\mu \mathrm{S} / \mathrm{cm})\end{array}$ & $\begin{array}{c}\mathrm{pH} \\
(\mathrm{S} . \mathrm{U} .) \\
\end{array}$ & $\begin{array}{c}\text { Redox } \\
(\mathrm{mV})\end{array}$ & $\begin{array}{l}\text { Temp } \\
\left({ }^{\circ} \mathrm{C}\right)\end{array}$ & $\begin{array}{c}\text { Alkalinity } \\
(\mathrm{mg} / \mathrm{L} \text { as } \\
\left.\mathrm{CaCO}_{3}\right)\end{array}$ & $\begin{array}{c}\text { Hardness } \\
(\mathrm{mg} / \mathrm{L} \text { as } \\
\left.\mathrm{CaCO}_{3}\right)\end{array}$ \\
\hline Inflow & 1.8 & 7.7 & 0.4390 & 0.0771 & nd & 8140 & nd & nd & nd & 430 & 1160 \\
\hline $\mathrm{A} 1^{\mathrm{a}}$ & 1.5 & 7.2 & 0.4688 & 0.1090 & 7.62 & 8250 & 7.5 & 276 & 13.7 & 450 & 1180 \\
\hline $\mathrm{A} 2$ & 0.7 & 6.1 & 0.4114 & 0.0789 & 9.41 & 8290 & 7.82 & -280 & 12.6 & 475 & 1200 \\
\hline A3 & 0.3 & 5.3 & 0.4129 & 0.0732 & 9.88 & 8390 & 7.59 & -254 & 11.3 & 366 & 1440 \\
\hline A4 & 0.1 & 2.1 & 0.3704 & 0.0509 & 8.3 & 8470 & 7.62 & 335 & 11.6 & 416 & 1440 \\
\hline B1 & 1 & 7.1 & 0.4141 & 0.1799 & 7.58 & 8250 & 6.87 & 315 & 10.3 & 422 & 1440 \\
\hline B2 & 0.9 & 5.6 & 0.4747 & 0.0785 & 6.6 & 8340 & 7.88 & -270 & 11.7 & 418 & 1260 \\
\hline B3 & 0.2 & 4.3 & 0.4113 & 0.0735 & 7.25 & 8430 & 7.1 & -161 & 12 & 422 & 1260 \\
\hline B4 & $<0.03$ & 1.9 & 0.3386 & 0.0338 & 7.11 & 8410 & 6.79 & 240 & 11.5 & 402 & 1440 \\
\hline $\mathrm{C} 1$ & 1.4 & 7.2 & 0.451 & 0.0790 & 7.6 & 8290 & 6.9 & 222 & 11.6 & 433 & 1260 \\
\hline $\mathrm{C} 2$ & 0.7 & 6.1 & 0.4532 & 0.0638 & 8.9 & 8350 & 7.8 & -183 & 10.4 & 450 & 1440 \\
\hline $\mathrm{C} 3$ & 0.2 & 4.7 & 0.3064 & 0.0442 & 5.61 & 8380 & 7.45 & -262 & 11.2 & 362 & 1420 \\
\hline $\mathrm{C} 4$ & $<0.03$ & 2.5 & 0.2397 & 0.0215 & 7.64 & 8390 & 7.67 & 176 & 11.5 & 340 & 900 \\
\hline D1 & 1.1 & 7.2 & 0.4492 & 0.0646 & 8.23 & 8310 & 6.82 & 280 & 11.8 & 368 & 1260 \\
\hline D2 & 0.7 & 6.4 & 0.4167 & 0.0417 & 7.6 & 8370 & 7.2 & -238 & 10.8 & 374 & 1080 \\
\hline D3 & 0.1 & 5.9 & 0.3667 & 0.0209 & 6.3 & 8440 & 7.34 & -212 & 11.1 & 312 & 900 \\
\hline D4 & $<0.03$ & 3.9 & 0.2189 & 0.0186 & 6.2 & 8420 & 7.18 & 212 & 11.3 & 280 & 1020 \\
\hline
\end{tabular}

nd- not determined

Cd bdl $(0.000025 \mathrm{mg} / \mathrm{L})$, Se bdl $(0.0002 \mathrm{mg} / \mathrm{L})$

${ }^{a}$ Sampling location format is series $(A)$, cell (1), etc., (Figure 1). 


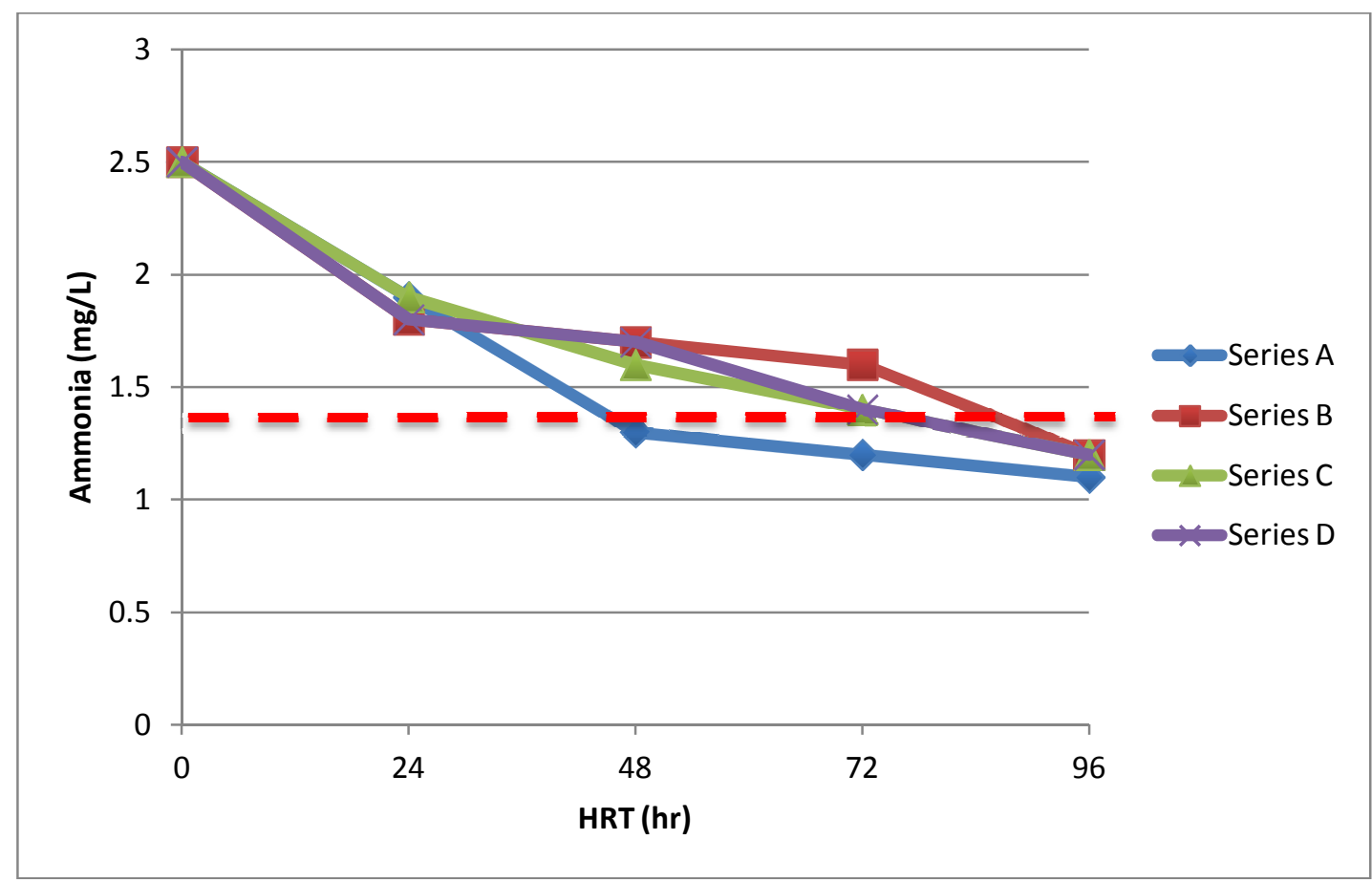

Figure 62. Ammonia concentration during sampling period $6(11 / 15 / 12)$ with a targeted performance goal of $1.4 \mathrm{mg} / \mathrm{L}$ (red dashed line).

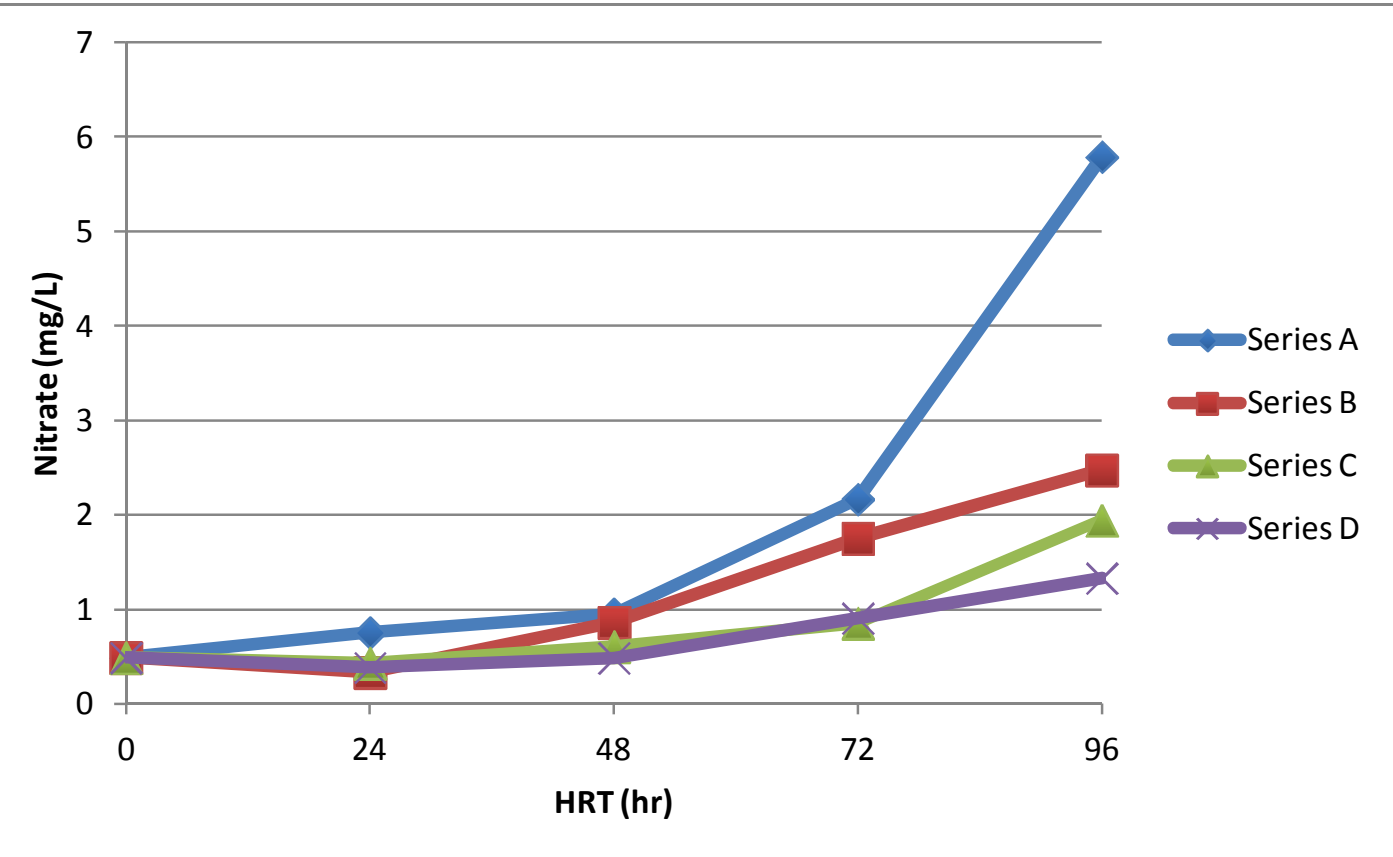

Figure 63. Nitrate concentrations during sampling period $6(11 / 15 / 12)$. The nitrate concentrations measured in the outflow were greater than what would be produced if all the ammonia in the inflow were converted to nitrate, indicating that ammonia in the inflow was removed as well as additional ammonia produced in the system. 


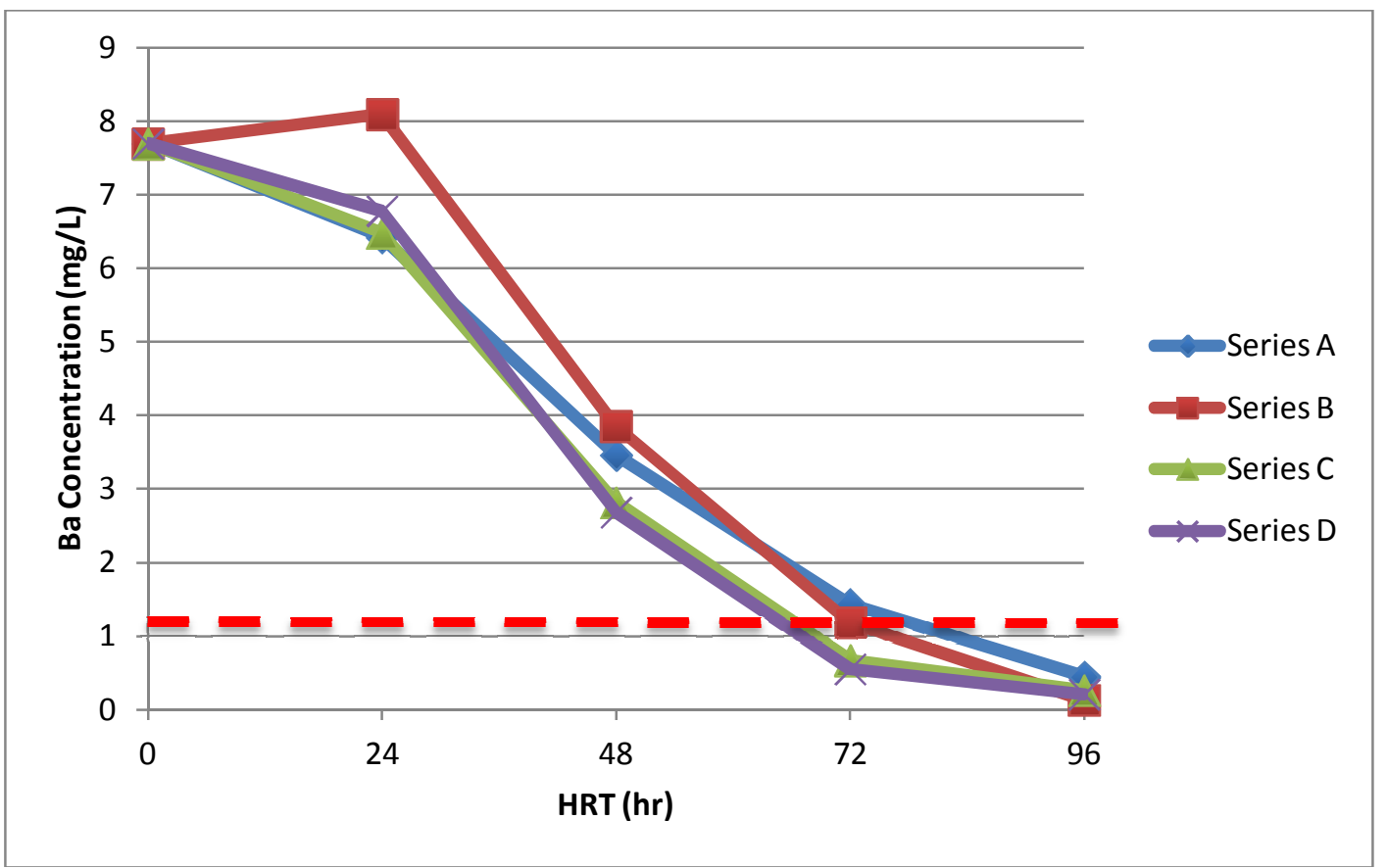

Figure 64. Barium concentration in sampling period $1(9 / 6 / 12)$ with a targeted performance goal of $1.0 \mathrm{mg} \mathrm{Ba} / \mathrm{L}$ (red dashed line).

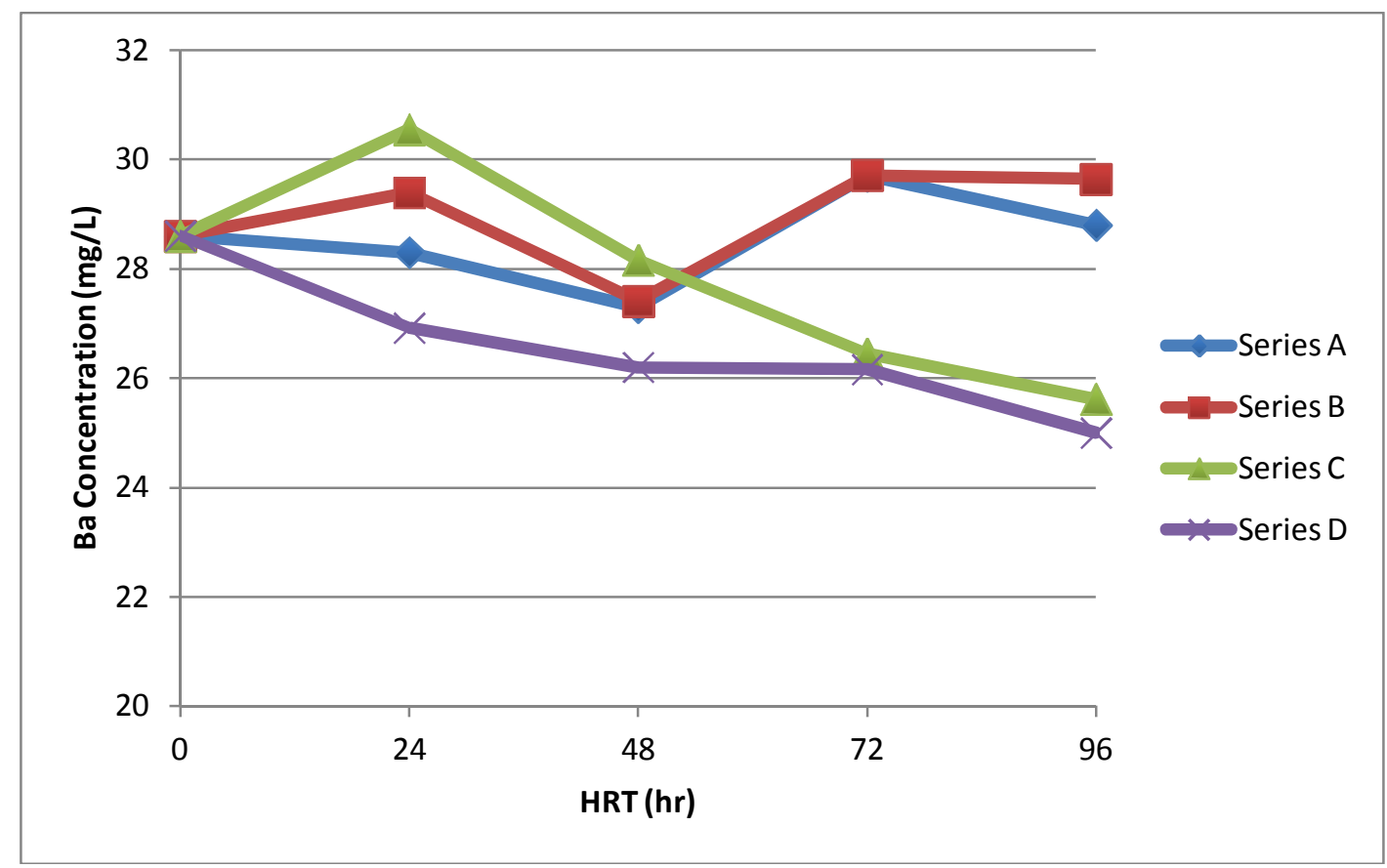

Figure 65. Barium concentration in sampling period 6 (11/15/12). Lack of decrease in Ba concentration is attributed to an insufficient amount of sulfate available, which was confirmed by measurement of sulfate concentration. Gypsum was added as a source of sulfate to all wetland cells on 11/29/12. 


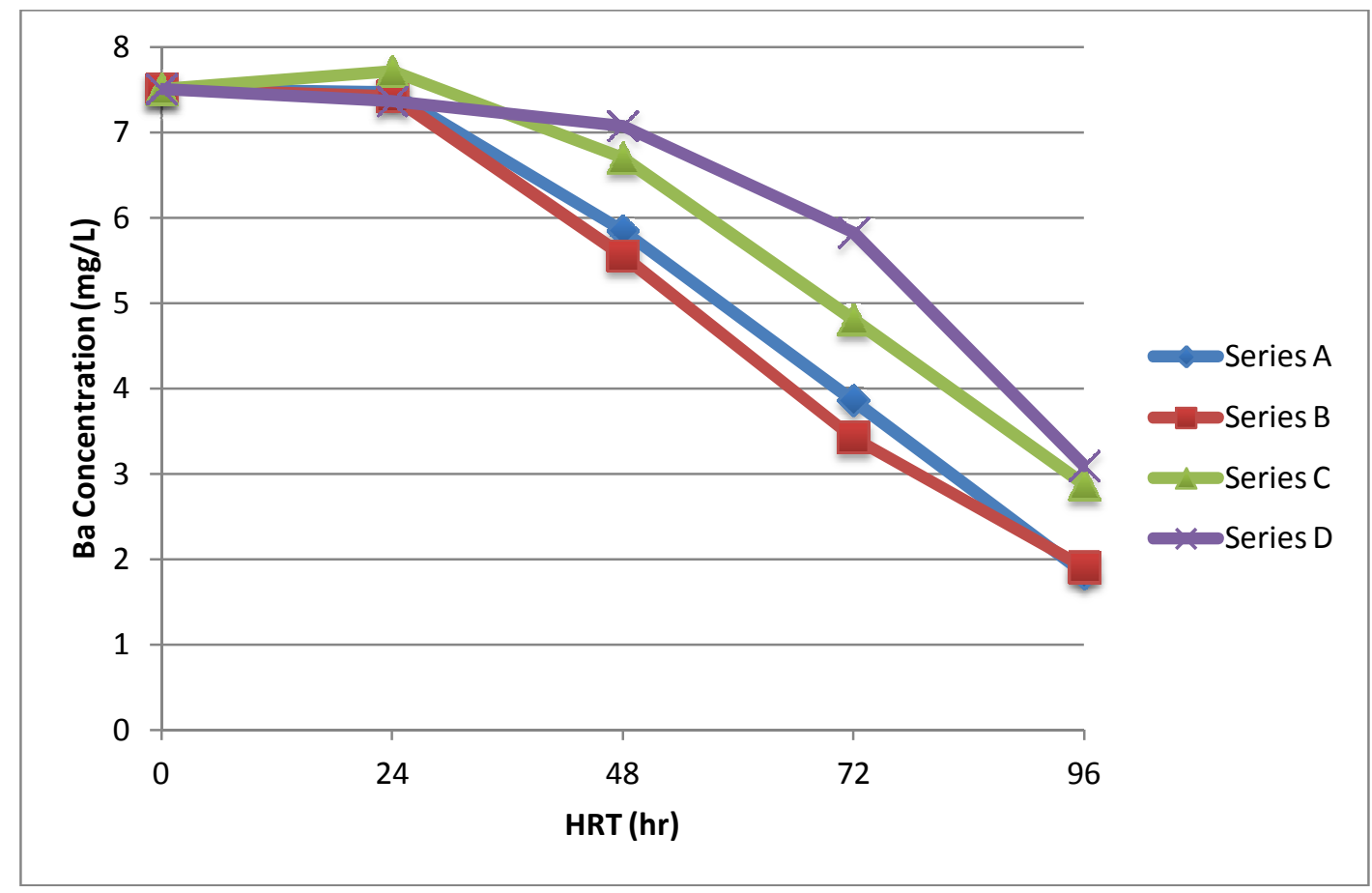

Figure 66. Barium concentration in sampling period 8 (12/13/12). Barium removal increased after the addition of gypsum to wetland cells on 11/29/12. 


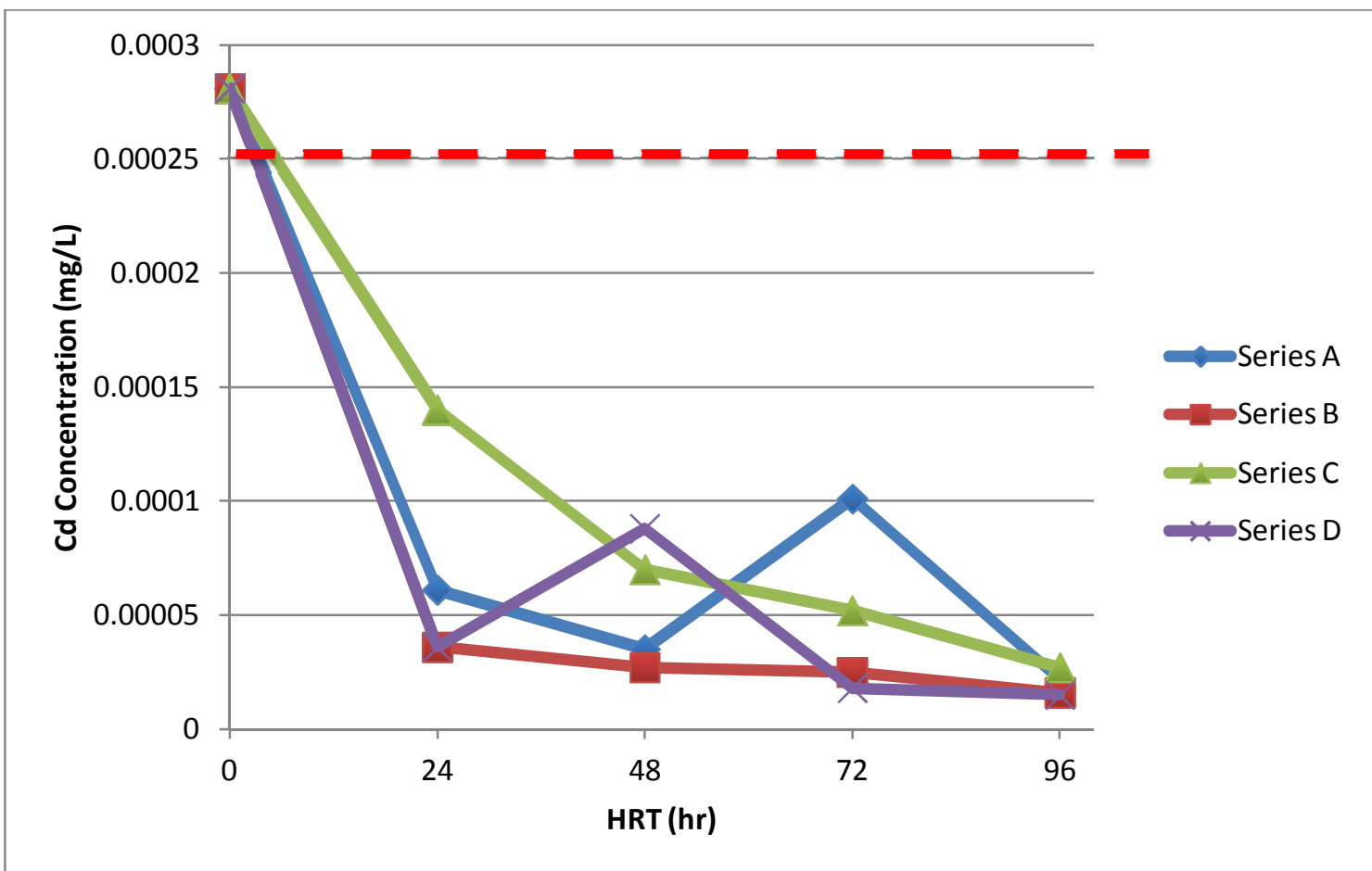

Figure 67. Cd concentration during sampling period $2(9 / 20 / 12)$ with a goal of $0.00025 \mathrm{mg}$ $\mathrm{Cd} / \mathrm{L}$ (red dashed line).

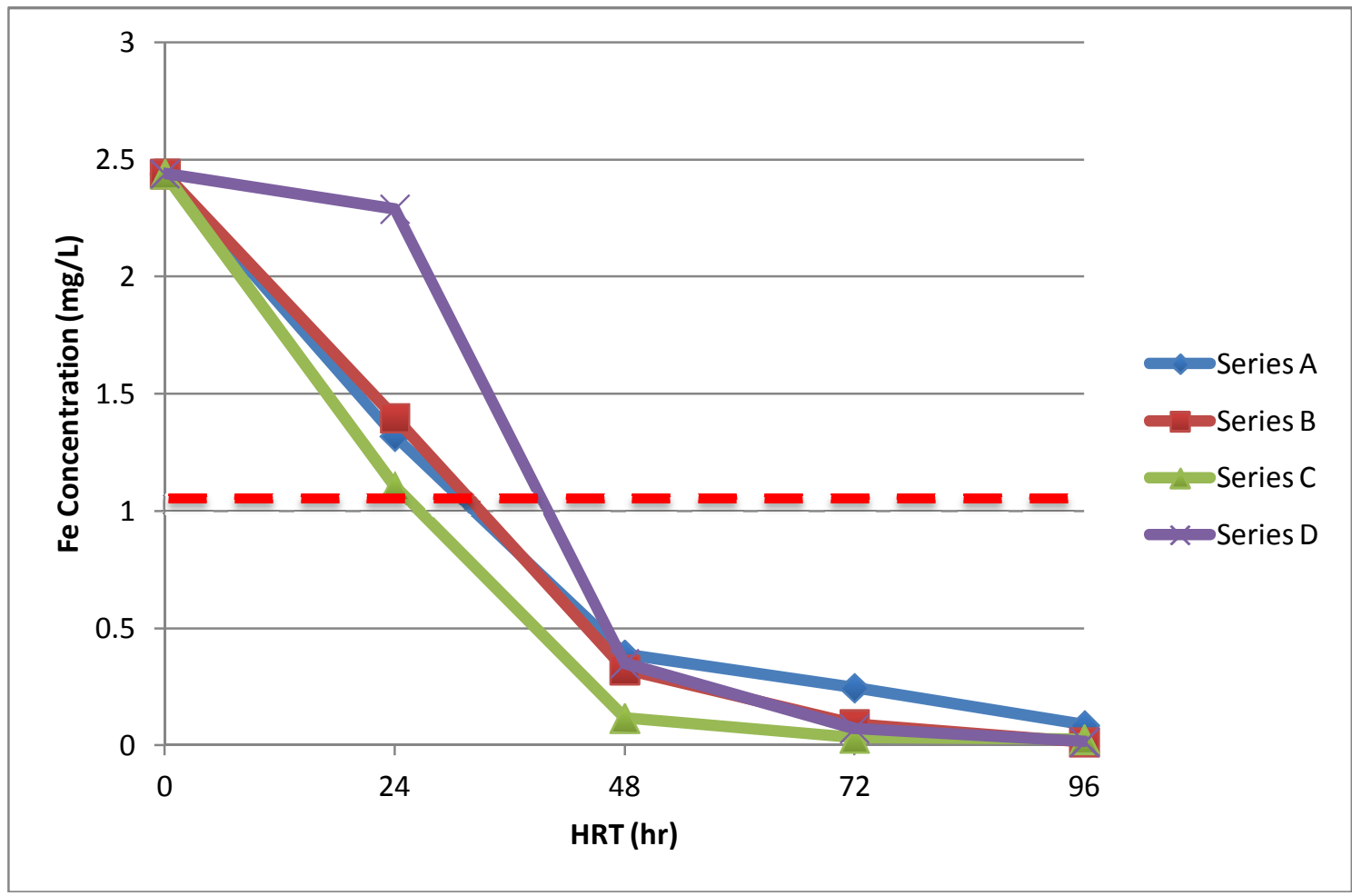

Figure 68. Fe concentration during sampling period $4(10 / 18 / 12)$ with a goal of $1.0 \mathrm{mg}$ $\mathrm{Fe} / \mathrm{L}$ (red dashed line). 
Table 83. Ammonia concentrations measured in CWTS inflow and outflows, removal efficiencies $(\%)$, and removal rate coefficients $\left(\mathrm{d}^{-1}\right)$. Removal efficiencies and removal rate coefficients were calculated using measured concentrations in the outflow (4-day HRT).

\begin{tabular}{|c|c|c|c|c|}
\hline & \multicolumn{3}{|c|}{ Ammonia } \\
\hline & & $\begin{array}{l}\text { Conc. } \\
\text { (mg/L) }\end{array}$ & $\begin{array}{c}\text { Removal } \\
(\%)\end{array}$ & $\begin{array}{l}\text { Rate Coef. } \\
\left(\mathrm{d}^{-1}\right)\end{array}$ \\
\hline \multirow{5}{*}{ 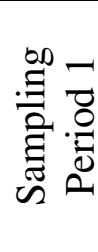 } & Inflow & 1.3 & -- & -- \\
\hline & Series A outflow & 2.3 & $\mathrm{nr}$ & $\mathrm{nr}$ \\
\hline & Series B outflow & 0.6 & 53.8 & 0.193 \\
\hline & Series C outflow & 0.3 & 76.9 & 0.367 \\
\hline & Series D outflow & 0.3 & 76.9 & 0.367 \\
\hline \multirow{5}{*}{ 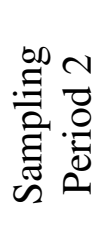 } & Inflow & 0.1 & -- & -- \\
\hline & Series A outflow & 0.1 & 0.0 & 0.000 \\
\hline & Series B outflow & $<0.03$ & $>70.0$ & $>0.301$ \\
\hline & Series C outflow & $<0.03$ & $>70.0$ & $>0.301$ \\
\hline & Series D outflow & $<0.03$ & $>70.0$ & $>0.301$ \\
\hline \multirow{5}{*}{ 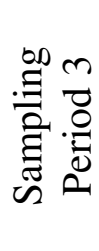 } & Inflow & 0.5 & -- & -- \\
\hline & Series A outflow & 0.1 & 80.0 & 0.402 \\
\hline & Series B outflow & $<0.03$ & $>94.0$ & $>0.703$ \\
\hline & Series C outflow & $<0.03$ & $>94.0$ & $>0.703$ \\
\hline & Series D outflow & $<0.03$ & $>94.0$ & $>0.703$ \\
\hline \multirow{5}{*}{ 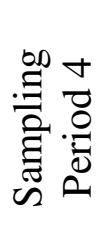 } & Inflow & 3.3 & -- & -- \\
\hline & Series A outflow & 1.4 & 57.6 & 0.214 \\
\hline & Series B outflow & 1.3 & 60.6 & 0.233 \\
\hline & Series C outflow & 0.9 & 72.7 & 0.325 \\
\hline & Series D outflow & 1.3 & 60.6 & 0.233 \\
\hline \multirow{5}{*}{ 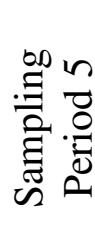 } & Inflow & 2.3 & -- & -- \\
\hline & Series A outflow & 0.1 & 95.7 & 0.784 \\
\hline & Series B outflow & 1 & 56.5 & 0.208 \\
\hline & Series C outflow & 0.9 & 60.9 & 0.235 \\
\hline & Series D outflow & 0.9 & 60.9 & 0.235 \\
\hline \multirow{5}{*}{ 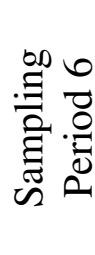 } & Inflow & 2.5 & -- & -- \\
\hline & Series A outflow & 1.1 & 56.0 & 0.205 \\
\hline & Series B outflow & 1.2 & 52.0 & 0.183 \\
\hline & Series C outflow & 1.2 & 52.0 & 0.183 \\
\hline & Series D outflow & 1.2 & 52.0 & 0.183 \\
\hline \multirow{5}{*}{ 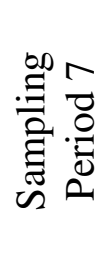 } & Inflow & 2.7 & -- & -- \\
\hline & Series A outflow & 0.8 & 70.4 & 0.304 \\
\hline & Series B outflow & 0.9 & 66.7 & 0.275 \\
\hline & Series C outflow & 0.8 & 70.4 & 0.304 \\
\hline & Series D outflow & 0.8 & 70.4 & 0.304 \\
\hline
\end{tabular}


Table 83. (continued)

\begin{tabular}{|c|c|c|c|c|}
\hline & \multicolumn{3}{|c|}{ Ammonia } \\
\hline & & $\begin{array}{l}\text { Conc. } \\
\text { (mg/L) }\end{array}$ & $\begin{array}{c}\text { Removal } \\
(\%)\end{array}$ & $\begin{array}{l}\text { Rate Coef. } \\
\qquad\left(\mathrm{d}^{-1}\right)\end{array}$ \\
\hline \multirow{5}{*}{ 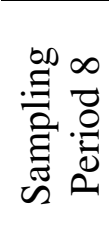 } & Inflow & 2.9 & -- & -- \\
\hline & Series A outflow & 0.4 & 86.2 & 0.495 \\
\hline & Series B outflow & 0.5 & 82.8 & 0.439 \\
\hline & Series C outflow & 0.2 & 93.1 & 0.669 \\
\hline & Series D outflow & 0.3 & 89.7 & 0.567 \\
\hline \multirow{5}{*}{ 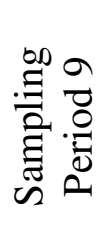 } & Inflow & 1.6 & -- & -- \\
\hline & Series A outflow & 0.1 & 93.8 & 0.693 \\
\hline & Series B outflow & 0.3 & 81.3 & 0.418 \\
\hline & Series C outflow & 0.1 & 93.8 & 0.693 \\
\hline & Series D outflow & 0.2 & 87.5 & 0.520 \\
\hline \multirow{5}{*}{ 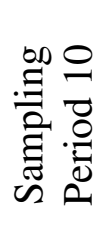 } & Inflow & 1.7 & -- & -- \\
\hline & Series A outflow & 0.2 & 88.2 & 0.535 \\
\hline & Series B outflow & 0.2 & 88.2 & 0.535 \\
\hline & Series C outflow & 0.2 & 88.2 & 0.535 \\
\hline & Series D outflow & 0.3 & 82.4 & 0.434 \\
\hline \multirow{5}{*}{ 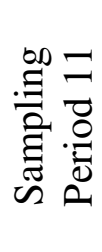 } & Inflow & 1.5 & -- & -- \\
\hline & Series A outflow & 0.6 & 60.0 & 0.229 \\
\hline & Series B outflow & 1.3 & 13.3 & 0.036 \\
\hline & Series C outflow & 1 & 33.3 & 0.101 \\
\hline & Series D outflow & 0.4 & 73.3 & 0.330 \\
\hline \multirow{5}{*}{ 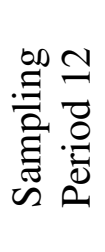 } & Inflow & 2 & -- & -- \\
\hline & Series A outflow & 1.2 & 40.0 & 0.128 \\
\hline & Series B outflow & 1.3 & 35.0 & 0.108 \\
\hline & Series C outflow & 1.2 & 40.0 & 0.128 \\
\hline & Series D outflow & 0.8 & 60.0 & 0.229 \\
\hline \multirow{5}{*}{ 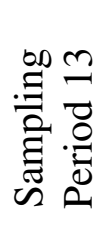 } & Inflow & 1.5 & -- & -- \\
\hline & Series A outflow & 0.1 & 93.3 & 0.677 \\
\hline & Series B outflow & 0.4 & 73.3 & 0.330 \\
\hline & Series C outflow & 0.2 & 86.7 & 0.504 \\
\hline & Series D outflow & 0.1 & 93.3 & 0.677 \\
\hline \multirow{5}{*}{ 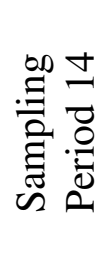 } & Inflow & 1.8 & -- & -- \\
\hline & Series A outflow & 0.1 & 94.4 & 0.723 \\
\hline & Series B outflow & $<0.03$ & $>98.3$ & $>1.024$ \\
\hline & Series C outflow & $<0.03$ & $>98.3$ & $>1.024$ \\
\hline & Series D outflow & $<0.03$ & $>98.3$ & $>1.024$ \\
\hline
\end{tabular}

nr- No removal occurred. 
Table 84. Barium concentrations measured in CWTS inflow and outflows, removal efficiencies (\%), and removal rate coefficients $\left(\mathrm{d}^{-1}\right)$. Removal efficiencies and removal rate coefficients were calculated using measured concentrations in the outflow (4-day HRT).

\begin{tabular}{|c|c|c|c|c|}
\hline & \multicolumn{3}{|c|}{ Barium } \\
\hline & & $\begin{array}{l}\text { Conc. } \\
\text { (mg/L) }\end{array}$ & $\begin{array}{c}\text { Removal } \\
(\%)\end{array}$ & $\begin{array}{l}\text { Rate Coef. } \\
\qquad\left(\mathrm{d}^{-1}\right)\end{array}$ \\
\hline \multirow{5}{*}{ 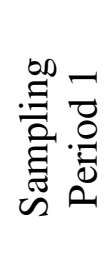 } & Inflow & 7.7 & -- & -- \\
\hline & Series A outflow & 0.45 & 94.2 & 0.710 \\
\hline & Series B outflow & 0.13 & 98.3 & 1.020 \\
\hline & Series C outflow & 0.26 & 96.6 & 0.847 \\
\hline & Series D outflow & 0.21 & 97.3 & 0.900 \\
\hline \multirow{5}{*}{ 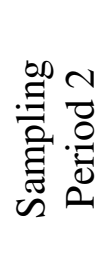 } & Inflow & 6.1 & -- & -- \\
\hline & Series A outflow & 0.54 & 91.1 & 0.606 \\
\hline & Series B outflow & 0.43 & 93.0 & 0.663 \\
\hline & Series C outflow & 0.31 & 94.9 & 0.745 \\
\hline & Series D outflow & 0.24 & 96.1 & 0.809 \\
\hline \multirow{5}{*}{ 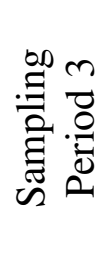 } & Inflow & 9.3 & -- & -- \\
\hline & Series A outflow & 0.28 & 97.0 & 0.876 \\
\hline & Series B outflow & 0.17 & 98.2 & 1.000 \\
\hline & Series C outflow & 0.82 & 91.2 & 0.607 \\
\hline & Series D outflow & 0.39 & 95.8 & 0.793 \\
\hline \multirow{5}{*}{ 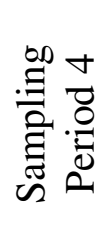 } & Inflow & 17 & -- & -- \\
\hline & Series A outflow & 1.2 & 92.9 & 0.663 \\
\hline & Series B outflow & 0.91 & 94.6 & 0.732 \\
\hline & Series C outflow & 0.59 & 96.5 & 0.840 \\
\hline & Series D outflow & 0.42 & 97.5 & 0.925 \\
\hline \multirow{5}{*}{ 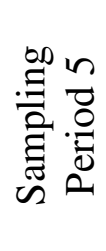 } & Inflow & 13.2 & -- & -- \\
\hline & Series A outflow & 1.4 & 89.4 & 0.561 \\
\hline & Series B outflow & 1.1 & 91.7 & 0.621 \\
\hline & Series C outflow & 0.9 & 93.2 & 0.671 \\
\hline & Series D outflow & 0.61 & 95.4 & 0.769 \\
\hline \multirow{5}{*}{ 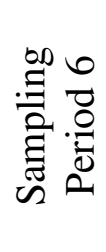 } & Inflow & 28.6 & -- & -- \\
\hline & Series A outflow & 28.8 & $\mathrm{nr}$ & $\mathrm{nr}$ \\
\hline & Series B outflow & 29.6 & $\mathrm{nr}$ & $\mathrm{nr}$ \\
\hline & Series C outflow & 25.6 & 10.5 & 0.028 \\
\hline & Series D outflow & 25 & 12.6 & 0.034 \\
\hline \multirow{5}{*}{ 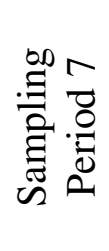 } & Inflow & 12.6 & -- & -- \\
\hline & Series A outflow & 14.6 & $\mathrm{nr}$ & $\mathrm{nr}$ \\
\hline & Series B outflow & 13.9 & $\mathrm{nr}$ & $\mathrm{nr}$ \\
\hline & Series C outflow & 15.5 & $\mathrm{nr}$ & $\mathrm{nr}$ \\
\hline & Series D outflow & 14 & $\mathrm{nr}$ & $\mathrm{nr}$ \\
\hline
\end{tabular}


Table 84. (continued)

\begin{tabular}{|c|c|c|c|c|}
\hline & \multicolumn{3}{|c|}{ Barium } \\
\hline & & $\begin{array}{l}\text { Conc. } \\
(\mathrm{mg} / \mathrm{L})\end{array}$ & $\begin{array}{c}\text { Removal } \\
(\%)\end{array}$ & $\begin{array}{c}\text { Rate Coef. } \\
\left(\mathrm{d}^{-1}\right)\end{array}$ \\
\hline \multirow{5}{*}{ 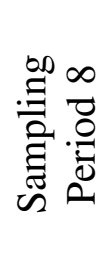 } & Inflow & 7.5 & -- & -- \\
\hline & Series A outflow & 1.8 & 76.0 & 0.357 \\
\hline & Series B outflow & 1.9 & 74.7 & 0.343 \\
\hline & Series C outflow & 2.9 & 61.3 & 0.238 \\
\hline & Series D outflow & 3.1 & 58.7 & 0.221 \\
\hline \multirow{5}{*}{ 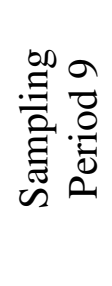 } & Inflow & 7.4 & -- & -- \\
\hline & Series A outflow & 1.1 & 85.1 & 0.477 \\
\hline & Series B outflow & 1.5 & 79.7 & 0.399 \\
\hline & Series C outflow & 3.1 & 58.1 & 0.218 \\
\hline & Series D outflow & 3.4 & 54.1 & 0.194 \\
\hline \multirow{5}{*}{ 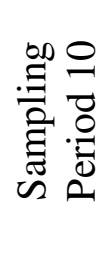 } & Inflow & 6.2 & -- & -- \\
\hline & Series A outflow & 2.4 & 61.3 & 0.237 \\
\hline & Series B outflow & 2.7 & 56.5 & 0.208 \\
\hline & Series C outflow & 1.4 & 77.4 & 0.372 \\
\hline & Series D outflow & 1.6 & 74.2 & 0.339 \\
\hline \multirow{5}{*}{ 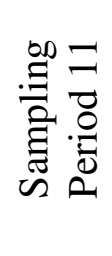 } & Inflow & 5.4 & -- & -- \\
\hline & Series A outflow & 2.7 & 50.0 & 0.173 \\
\hline & Series B outflow & 2.7 & 50.0 & 0.173 \\
\hline & Series C outflow & 2.9 & 46.3 & 0.155 \\
\hline & Series D outflow & 3.3 & 38.9 & 0.123 \\
\hline \multirow{5}{*}{ 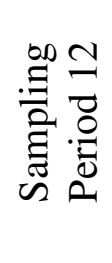 } & Inflow & 42.1 & -- & -- \\
\hline & Series A outflow & 2.4 & 94.3 & 0.716 \\
\hline & Series B outflow & 2.1 & 95.0 & 0.750 \\
\hline & Series C outflow & 2.4 & 94.3 & 0.716 \\
\hline & Series D outflow & 3.9 & 90.7 & 0.595 \\
\hline \multirow{5}{*}{ 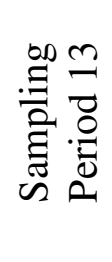 } & Inflow & 7.5 & -- & -- \\
\hline & Series A outflow & 2.9 & 61.3 & 0.238 \\
\hline & Series B outflow & 2.1 & 72.0 & 0.318 \\
\hline & Series C outflow & 3.1 & 58.7 & 0.221 \\
\hline & Series D outflow & 3.9 & 48.0 & 0.163 \\
\hline \multirow{5}{*}{ 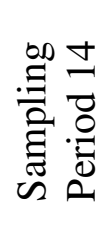 } & Inflow & 7.7 & -- & -- \\
\hline & Series A outflow & 2.1 & 72.7 & 0.325 \\
\hline & Series B outflow & 1.9 & 75.3 & 0.350 \\
\hline & Series C outflow & 2.5 & 67.5 & 0.281 \\
\hline & Series D outflow & 3.9 & 49.4 & 0.170 \\
\hline
\end{tabular}

nr- No removal occurred. 
Table 85. Cadmium concentrations measured in CWTS inflow and outflows, removal efficiencies $(\%)$, and removal rate coefficients $\left(\mathrm{d}^{-1}\right)$. Removal efficiencies and removal rate coefficients were calculated using measured concentrations in the outflow (4-day HRT). Cadmium concentrations were above the WQC in only the first two sampling periods.

\begin{tabular}{|c|c|c|c|c|}
\hline & \multicolumn{3}{|c|}{ Cadmium } \\
\hline & & $\begin{array}{l}\text { Conc. } \\
\text { (mg/L) }\end{array}$ & $\begin{array}{c}\text { Removal } \\
(\%)\end{array}$ & $\begin{array}{c}\text { Rate Coef. } \\
\left(\mathrm{d}^{-1}\right)\end{array}$ \\
\hline \multirow{5}{*}{ 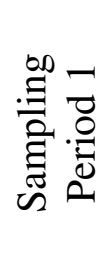 } & Inflow & 0.000108 & -- & -- \\
\hline & Series A outflow & $<0.000025$ & $>76.9$ & $>0.366$ \\
\hline & Series B outflow & 0.000034 & 68.5 & 0.289 \\
\hline & Series C outflow & 0.00003 & 72.2 & 0.320 \\
\hline & Series D outflow & 0.000107 & 0.9 & 0.002 \\
\hline \multirow{5}{*}{ 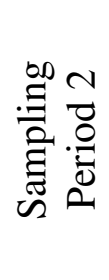 } & Inflow & 0.000281 & -- & -- \\
\hline & Series A outflow & $<0.000025$ & $>91.1$ & $>0.605$ \\
\hline & Series B outflow & $<0.000025$ & $>91.1$ & $>0.605$ \\
\hline & Series C outflow & 0.000027 & 90.4 & 0.586 \\
\hline & Series D outflow & $<0.000025$ & $>91.1$ & $>0.605$ \\
\hline
\end{tabular}

nr- No removal occurred. 
Table 86. Iron concentrations measured in CWTS inflow and outflows, removal efficiencies $(\%)$, and removal rate coefficients $\left(\mathrm{d}^{-1}\right)$. Removal efficiencies and removal rate coefficients were calculated using measured concentrations in the outflow (4-day HRT). Sampling period 3 is not included due to concentration of iron less than the WQC in inflow and outflow.

\begin{tabular}{|c|c|c|c|c|}
\hline & \multicolumn{3}{|c|}{ Iron } \\
\hline & & $\begin{array}{l}\text { Conc. } \\
\text { (mg/L) }\end{array}$ & $\begin{array}{c}\text { Removal } \\
(\%)\end{array}$ & $\begin{array}{c}\text { Rate Coef } \\
\left(\mathrm{d}^{-1}\right)\end{array}$ \\
\hline \multirow{5}{*}{ 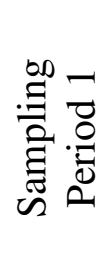 } & Inflow & 0.2758 & -- & -- \\
\hline & Series A outflow & 0.6978 & $\mathrm{nr}$ & $\mathrm{nr}$ \\
\hline & Series B outflow & 0.3139 & $\mathrm{nr}$ & $\mathrm{nr}$ \\
\hline & Series C outflow & 0.0846 & 69.3 & 0.295 \\
\hline & Series D outflow & 0.1755 & 36.4 & 0.113 \\
\hline \multirow{5}{*}{ 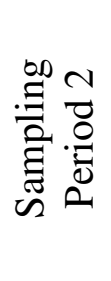 } & Inflow & 0.1006 & -- & -- \\
\hline & Series A outflow & 0.2032 & $\mathrm{nr}$ & $\mathrm{nr}$ \\
\hline & Series B outflow & 0.0138 & 86.3 & 0.496 \\
\hline & Series C outflow & 0.0093 & 90.8 & 0.596 \\
\hline & Series D outflow & 0.0162 & 83.9 & 0.456 \\
\hline \multirow{5}{*}{ 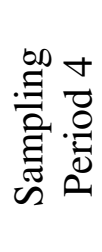 } & Inflow & 2.44 & -- & -- \\
\hline & Series A outflow & 0.088 & 96.4 & 0.831 \\
\hline & Series B outflow & 0.016 & 99.3 & 1.257 \\
\hline & Series C outflow & 0.028 & 98.9 & 1.117 \\
\hline & Series D outflow & 0.017 & 99.3 & 1.242 \\
\hline \multirow{5}{*}{ 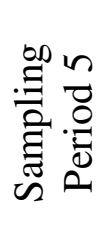 } & Inflow & 1.48 & -- & -- \\
\hline & Series A outflow & 0.047 & 96.8 & 0.862 \\
\hline & Series B outflow & 0.02 & 98.6 & 1.076 \\
\hline & Series C outflow & 0.331 & 77.6 & 0.374 \\
\hline & Series D outflow & 0.104 & 93.0 & 0.664 \\
\hline \multirow{5}{*}{ 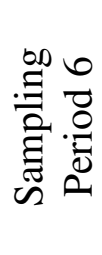 } & Inflow & 1.524 & -- & -- \\
\hline & Series A outflow & 0.119 & 92.2 & 0.637 \\
\hline & Series B outflow & 0.07 & 95.4 & 0.770 \\
\hline & Series C outflow & 0.123 & 91.9 & 0.629 \\
\hline & Series D outflow & 0.144 & 90.6 & 0.590 \\
\hline \multirow{5}{*}{ 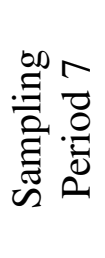 } & Inflow & 0.262 & -- & -- \\
\hline & Series A outflow & 0.109 & 58.4 & 0.219 \\
\hline & Series B outflow & 0.055 & 79.0 & 0.390 \\
\hline & Series C outflow & 0.089 & 66.0 & 0.270 \\
\hline & Series D outflow & 0.013 & 95.0 & 0.751 \\
\hline
\end{tabular}


Table 86. (continued)

\begin{tabular}{|c|c|c|c|c|}
\hline & \multicolumn{3}{|c|}{ Iron } \\
\hline & & $\begin{array}{l}\text { Conc. } \\
\text { (mg/L) }\end{array}$ & $\begin{array}{c}\text { Removal } \\
(\%)\end{array}$ & $\begin{array}{c}\text { Rate Coef. } \\
\left(\mathrm{d}^{-1}\right)\end{array}$ \\
\hline \multirow{5}{*}{ 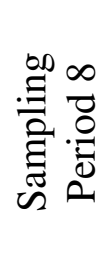 } & Inflow & 0.7842 & -- & -- \\
\hline & Series A outflow & 0.2168 & 72.4 & 0.321 \\
\hline & Series B outflow & 0.0990 & 87.4 & 0.517 \\
\hline & Series C outflow & 0.1481 & 81.1 & 0.417 \\
\hline & Series D outflow & 0.0554 & 92.9 & 0.662 \\
\hline \multirow{5}{*}{ 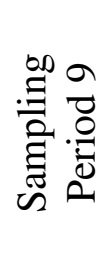 } & Inflow & 0.1316 & -- & -- \\
\hline & Series A outflow & 0.0912 & 30.7 & 0.092 \\
\hline & Series B outflow & 0.0444 & 66.2 & 0.272 \\
\hline & Series C outflow & 0.0675 & 48.7 & 0.167 \\
\hline & Series D outflow & 0.0060 & 95.4 & 0.771 \\
\hline \multirow{5}{*}{ 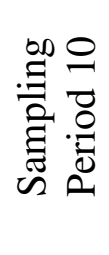 } & Inflow & 0.3554 & -- & -- \\
\hline & Series A outflow & 0.1308 & 63.2 & 0.250 \\
\hline & Series B outflow & 0.0559 & 84.2 & 0.462 \\
\hline & Series C outflow & 0.1144 & 67.8 & 0.283 \\
\hline & Series D outflow & 0.0371 & 89.6 & 0.565 \\
\hline \multirow{5}{*}{ 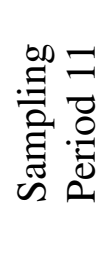 } & Inflow & 0.5258 & -- & -- \\
\hline & Series A outflow & 0.2035 & 61.3 & 0.237 \\
\hline & Series B outflow & 0.2382 & 54.7 & 0.198 \\
\hline & Series C outflow & 0.161 & 69.4 & 0.296 \\
\hline & Series D outflow & 0.1473 & 72.0 & 0.318 \\
\hline \multirow{5}{*}{ 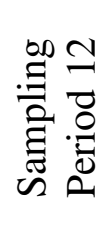 } & Inflow & 0.5589 & -- & -- \\
\hline & Series A outflow & 0.3387 & 39.4 & 0.125 \\
\hline & Series B outflow & 0.324 & 42.0 & 0.136 \\
\hline & Series C outflow & 0.2439 & 56.4 & 0.207 \\
\hline & Series D outflow & 0.1852 & 66.9 & 0.276 \\
\hline \multirow{5}{*}{ 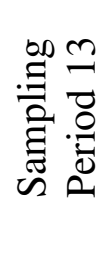 } & Inflow & 0.5386 & -- & -- \\
\hline & Series A outflow & 0.3061 & 43.2 & 0.141 \\
\hline & Series B outflow & 0.3229 & 40.0 & 0.128 \\
\hline & Series C outflow & 0.2607 & 51.6 & 0.181 \\
\hline & Series D outflow & 0.2479 & 54.0 & 0.194 \\
\hline \multirow{5}{*}{ 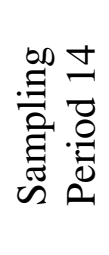 } & Inflow & 0.439 & -- & -- \\
\hline & Series A outflow & 0.3704 & 15.6 & 0.042 \\
\hline & Series B outflow & 0.3386 & 22.9 & 0.065 \\
\hline & Series C outflow & 0.2397 & 45.4 & 0.151 \\
\hline & Series D outflow & 0.2189 & 50.1 & 0.174 \\
\hline
\end{tabular}

nr- No removal occurred. 
Table 87. Manganese concentrations measured in CWTS inflow and outflows, removal efficiencies $(\%)$, and removal rate coefficients $\left(\mathrm{d}^{-1}\right)$. Removal efficiencies and removal rate coefficients were calculated using measured concentrations in the outflow (4-day HRT). Manganese concentrations were below detection limit during sampling periods 3-7, and are omitted from the table.

\begin{tabular}{|c|c|c|c|c|}
\hline & \multicolumn{3}{|c|}{ Manganese } \\
\hline & & $\begin{array}{l}\text { Conc. } \\
(\mathrm{mg} / \mathrm{L})\end{array}$ & $\begin{array}{c}\text { Removal } \\
(\%)\end{array}$ & $\begin{array}{c}\text { Rate Coef. } \\
\left(\mathrm{d}^{-1}\right)\end{array}$ \\
\hline \multirow{5}{*}{ 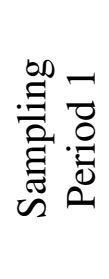 } & Inflow & 0.1244 & -- & -- \\
\hline & Series A outflow & 2.272 & $\mathrm{nr}$ & $\mathrm{nr}$ \\
\hline & Series B outflow & 2.658 & $\mathrm{nr}$ & $\mathrm{nr}$ \\
\hline & Series C outflow & 0.6731 & $\mathrm{nr}$ & $\mathrm{nr}$ \\
\hline & Series D outflow & 1.019 & $\mathrm{nr}$ & $\mathrm{nr}$ \\
\hline \multirow{5}{*}{ 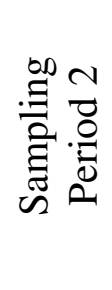 } & Inflow & 0.0200 & -- & -- \\
\hline & Series A outflow & 0.0372 & $\mathrm{nr}$ & $\mathrm{nr}$ \\
\hline & Series B outflow & 0.0028 & 86.0 & 0.492 \\
\hline & Series C outflow & 0.0146 & 27.0 & 0.079 \\
\hline & Series D outflow & 0.0541 & $\mathrm{nr}$ & $\mathrm{nr}$ \\
\hline \multirow{5}{*}{ 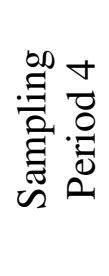 } & Inflow & 0.169 & -- & -- \\
\hline & Series A outflow & $<0.0014$ & $>99.2$ & $>1.198$ \\
\hline & Series B outflow & $<0.0014$ & $>99.2$ & $>1.198$ \\
\hline & Series C outflow & $<0.0014$ & $>99.2$ & $>1.198$ \\
\hline & Series D outflow & $<0.0014$ & $>99.2$ & $>1.198$ \\
\hline \multirow{5}{*}{ 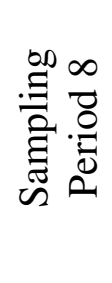 } & Inflow & 0.0299 & -- & -- \\
\hline & Series A outflow & $<0.0014$ & $>95.3$ & $>0.766$ \\
\hline & Series B outflow & $<0.0014$ & $>95.3$ & $>0.766$ \\
\hline & Series C outflow & $<0.0014$ & $>95.3$ & $>0.766$ \\
\hline & Series D outflow & $<0.0014$ & $>95.3$ & $>0.766$ \\
\hline \multirow{5}{*}{ 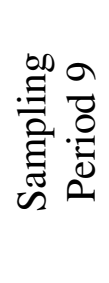 } & Inflow & 0.0341 & -- & -- \\
\hline & Series A outflow & 0.0274 & 19.7 & 0.055 \\
\hline & Series B outflow & 0.0079 & 76.8 & 0.366 \\
\hline & Series C outflow & $<0.0014$ & $>95.9$ & $>0.798$ \\
\hline & Series D outflow & 0.0111 & 67.4 & 0.281 \\
\hline \multirow{5}{*}{ 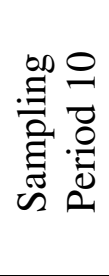 } & Inflow & 0.0385 & -- & -- \\
\hline & Series A outflow & 0.0377 & 2.3 & 0.006 \\
\hline & Series B outflow & $<0.0014$ & $>96.4$ & $>0.829$ \\
\hline & Series C outflow & 0.0335 & 13.0 & 0.035 \\
\hline & Series D outflow & $<0.0014$ & $>96.4$ & $>0.829$ \\
\hline
\end{tabular}

nr- No removal occurred. 
Table 87. (continued)

\begin{tabular}{|c|c|c|c|c|}
\hline & \multicolumn{3}{|c|}{ Manganese } \\
\hline & & $\begin{array}{l}\text { Conc. } \\
\text { (mg/L) }\end{array}$ & $\begin{array}{c}\text { Removal } \\
(\%)\end{array}$ & $\begin{array}{l}\text { Rate Coef. } \\
\left(\mathrm{d}^{-1}\right)\end{array}$ \\
\hline \multirow{5}{*}{ 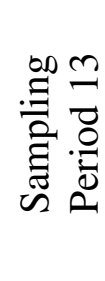 } & Inflow & 0.0659 & -- & -- \\
\hline & Series A outflow & 0.0448 & 32.0 & 0.096 \\
\hline & Series B outflow & 0.0431 & 34.6 & 0.106 \\
\hline & Series $C$ outflow & 0.0382 & 42.0 & 0.136 \\
\hline & Series D outflow & 0.0279 & 57.7 & 0.215 \\
\hline \multirow{5}{*}{ 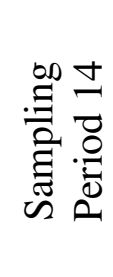 } & Inflow & 0.0771 & -- & -- \\
\hline & Series A outflow & 0.0509 & 34.0 & 0.104 \\
\hline & Series B outflow & 0.0338 & 56.1 & 0.206 \\
\hline & Series C outflow & 0.0215 & 72.1 & 0.319 \\
\hline & Series D outflow & 0.0186 & 75.9 & 0.355 \\
\hline
\end{tabular}

nr- No removal occurred. 


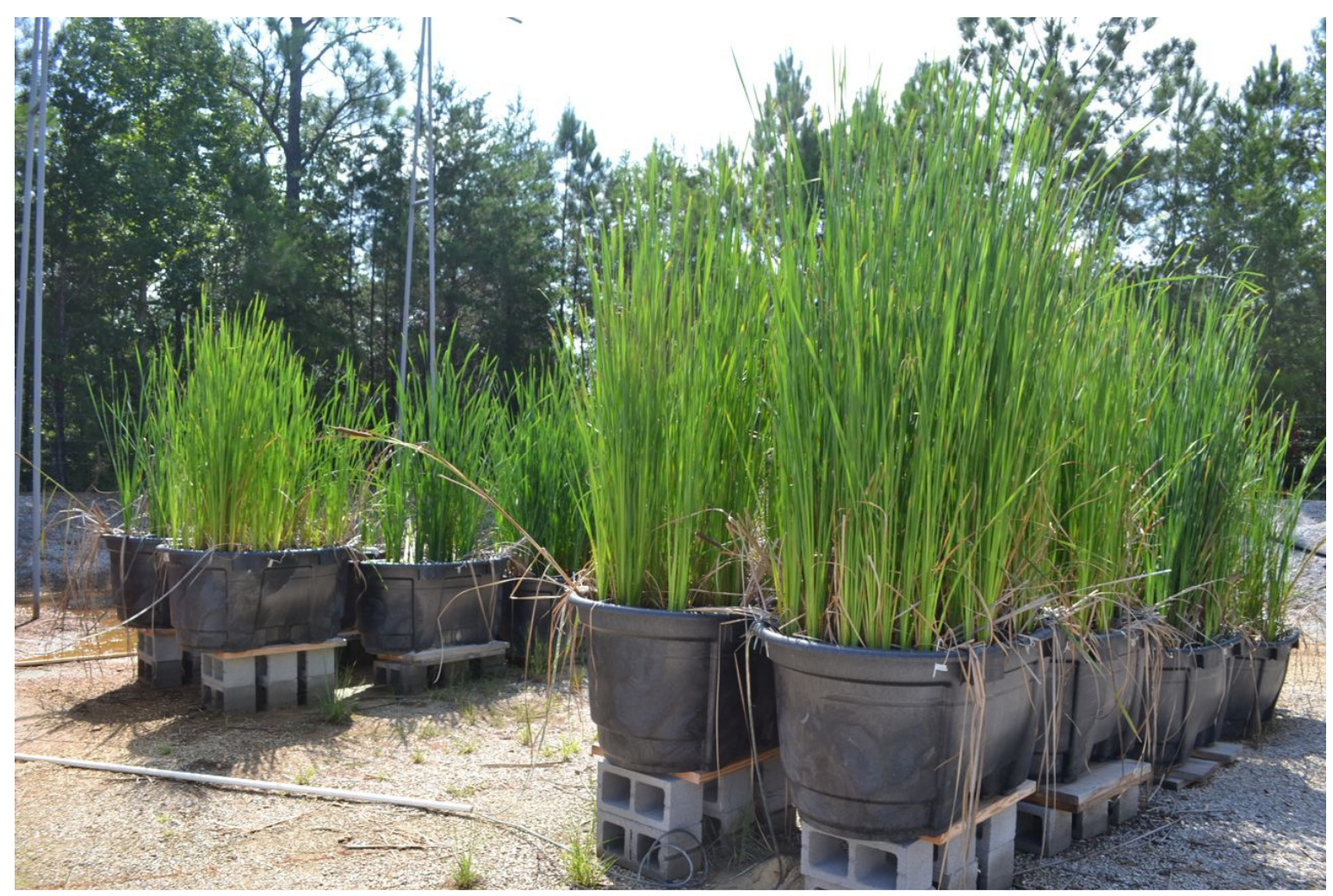

Figure 69. Demonstration wetland treatment system on 9/7/12, 14 weeks after construction. 
Apply Results to Water Management and Technology Replication (Task 9)

\section{Application to Various Geologic Basins and Locations}

A strategic literature review and analysis of field-collected water samples completed during Task 2 revealed that produced water composition varies from basin to basin and even from well to well within the same basin. Therefore, targeted constituents considered for treatment during the pilot-scale and demonstration phases of the project (Phases II and III) consisted of constituents that could potentially limit beneficial use of produced water at many locations. These targeted constituents, identified as COCs during Task 3, were addressed for treatment by specifically designed pilot-scale CWTSs in Phase II and included dissolved solids, metals, metalloids, organic compounds, and nitrogenous compounds.

Results from Phase II demonstrated successful treatment of the targeted COCs found in produced water including $\mathrm{Fe}, \mathrm{Mn}, \mathrm{Zn}, \mathrm{Ni}, \mathrm{Cd}, \mathrm{Cu}, \mathrm{Se}$, ammonia, nitrate, oil and grease, and low molecular weight organics to meet beneficial use criteria. Using the design principles established during Phase II, an on-site demonstration CWTS was constructed to remove $\mathrm{Ba}, \mathrm{Cd}, \mathrm{Fe}, \mathrm{Mn}$, and ammonia from coal-bed methane produced water generated from the Black Warrior basin in Alabama. The demonstration CWTS was successful for treating the COCs, thus renovating the produced water to meet stringent discharge limits and guideline criteria for crop irrigation and livestock watering. Successful transfer of CWTS design principles from pilot-scale to demonstration supports the applicability of experimental results from this study for design of future CWTSs.

CWTSs can be tailored to suit a variety of needs presented in areas that have produced waters of differing geologic origins. For instance, coal-bed methane water from the Powder River basin containing elevated concentrations of metals (e.g. Fe, $\mathrm{Cu}, \mathrm{Zn}$, etc.) and $\mathrm{Se}$ may be treated with a modular CWTS based on the design principles established in Phase II. Oxidizing CWTS cells can be used to precipitate and sequester Fe as insoluble iron(III) oxyhydroxide. Reducing CWTS cells can be assembled to promote dissimilatory sulfate reduction and sulfide precipitation of $\mathrm{Cu}$ and $\mathrm{Zn}$ as well as microbial reduction and precipitation of elemental Se. Removal rate coefficients measured in Phase II can be used to predict required treatment capacity for a given hydraulic loading that is site- or situation-dependent.

Additional treatment technologies can be incorporated with CWTSs (forming hybrid systems) to expand treatability and beneficial use options. As demonstrated in Phase II, oil-water separators can be used to pretreat produced water prior to its introduction to CWTSs to remove nonaqueous phase organic compounds and oil. The separated oil can then be collected and sold to recover operational and maintenance costs. Reverse osmosis can be used to remove dissolved salts from brackish and saline produced waters so they may be suitable for treatment by CWTSs and subsequently available for salinity-sensitive beneficial use options such as crop irrigation. Hypersaline produced water from some geologic formations such as the Marcellus Shale and Mississippi Lime may not be suitable for treatment due to the current limitations of desalinization technologies, therefore requiring disposal by deep-well injection. 


\section{Beneficial Use Selection}

Two different approaches can be used as the basis for designing CWTSs: a water-specific approach or a site-specific approach. With a water-specific approach, a CWTS can be designed to target all treatable COCs determined during characterization of produced water samples. For example, the pilot-scale CWTSs used in Phase II and demonstration CWTS used in Phase III were designed to meet the most stringent use criteria for each targeted COC. By targeting the most stringent criteria for each COC, produced water can be treated to permit beneficial use for as many different applications as possible. For instance, treated produced water from the Black Warrior basin in Alabama may be used for crop irrigation, livestock watering, industrial process water, power-plant cooling water, aquifer recharge, habitat restoration, and stream augmentation. A water-specific approach can be appropriate when there is a local demand for water for a variety of uses, when the beneficial use is unknown or may change through time, and/or when the produced water contains COCs that are not effectively removed by CWTSs (e.g. total dissolved solids). Because beneficial use demands are subject to change as a result of several factors including changes in seasons and changes in local land use, treating produced water to meet a multitude of beneficial use options helps ensure that the treated water can be used to supplement both current and future needs.

With a site-specific approach, a CWTS can be designed to treat produced water to meet beneficial use criteria for specific local needs. For example, treating produced water generated in urban areas to meet use criteria for crop irrigation and livestock watering in urban areas is not practical. Instead, the produced water may be treated to meet different use criteria more appropriate for local needs such as industrial process water. A site-specific approach can be more applicable in areas where local needs are not predicted to change over the lifetime of the CWTS.

\section{CWTS Treatment Cost Comparison}

The most feasible approach for treating site-specific produced water depends on both capital and operational costs and the ability of the treatment system to meet guideline criteria for beneficial use. Costs of CWTS construction, operation, and maintenance can vary widely depending on the location. These costs are affected primarily by treatment objectives, local terrain, and bottomliner requirements. Mooney and Murray Gulde (2008) found that costs for constructing CWTSs are commonly 50 to $70 \%$ less than for conventional treatment systems, with CWTS operation and maintenance costs between $\$ 0.25$ and $\$ 0.80$ per 1000 gallons of water treated. At a location in USDOE's Savannah River Site, where nearly 1 million gallons of water per day require treatment, estimated costs for conventional treatment systems for metal removal (ion exchange, chemical precipitation, source removal, etc.) proved to be too high for the water volume and treatment criteria (Nelson and Gladden, 2008). CWTSs were constructed as a low-cost alternative and resulted in operation and maintenance costs of less than $\$ 50,000$ per year, which is less than \$0.14 per 1000 gallons of water treated (Nelson and Gladden, 2008). Jackson and Myers (2003) provided cost estimates for disposal methods of produced water, and ALL Consulting (2012) provided information on treatment processes being utilized in the shale gas industry as part of a "Water Treatment Catalog" (Table 84). Cost estimates for different 
Table 84. Produced water treatment and disposal methods and estimated costs.

\begin{tabular}{|c|c|c|c|}
\hline Method & $\begin{array}{l}\text { Estimated cost } \\
(\$ / \mathrm{bbl})\end{array}$ & Limitation & Benefits \\
\hline Surface discharge $^{1}$ & $0.01-0.08$ & Stringent standards ${ }^{3}$ & Livestock, wildlife, irrigation \\
\hline Secondary oil recovery ${ }^{1}$ & $0.05-1.25$ & Infrastructure & Increase oil production \\
\hline Evaporation pits ${ }^{1}$ & $0.01-0.80$ & Sequestering from wildlife/soil contamination & Simple, passive management strategy \\
\hline Commercial water hauling ${ }^{1}$ & $0.01-5.50$ & Distance, safety issues & - \\
\hline Disposal wells $^{1}$ & $0.05-2.65$ & Strict environmental regulations & - \\
\hline Freeze-thaw evaporation ${ }^{1}$ & $2.65-5.00$ & Regional climate & Water/salt separation \\
\hline Constructed wetland $^{1}$ & $0.001-2.00$ & Land area, retention time requirement & $\begin{array}{l}\text { Low cost than many technologies; efficient } \\
\text { removal of dissolved and suspended } \\
\text { contaminants; low energy requirement }\end{array}$ \\
\hline $\begin{array}{l}\text { Induced air flotation for de- } \\
\text { oiling }^{1}\end{array}$ & 0.05 & CAPEX & Simple operation; durable ${ }^{3}$ \\
\hline $\begin{array}{l}\text { Anoxic/aerobic granular } \\
\text { activated carbon }{ }^{1}\end{array}$ & 0.083 & CAPEX & Simple technology $y^{3}$ \\
\hline Thermal evaporation $^{2}$ & $3.00-5.00$ & $\begin{array}{l}\text { Pretreatment required; not effective above } \\
\quad 80,000 \mathrm{mg} / \mathrm{L} \text { TDS }\end{array}$ & Effective for PW with $40,000-80,000 \mathrm{mg} / \mathrm{L} \mathrm{TDS}$ \\
\hline Reverse Osmosis $(\mathrm{RO})^{2}$ & 0.11 & 40 to $65 \%$ water recovery & Removes most ions \\
\hline Electrodialysis $^{2}$ & 0.15 & TDS 4,000-15,000 mg/L & More resistant to membrane fouling than $\mathrm{RO}$ \\
\hline Ion Exchange ${ }^{2}$ & $0.05-0.20$ & $\begin{array}{l}\text { TDS must be }<5,000 \mathrm{mg} / \mathrm{L} \text {; may require } \\
\text { pretreatment }\end{array}$ & Low energy requirement \\
\hline UV Light $^{2}$ & $0.60-0.75$ & May require use of biocides & Can disinfect PW \\
\hline Ozone $^{2}$ & $0.60-0.75$ & Complex chemistry; toxicity issues & $\begin{array}{l}\text { Removes bacteria without producing waste } \\
\text { byproduct }\end{array}$ \\
\hline
\end{tabular}

bbl = barrel of treated water $(1 \mathrm{bbl}=42$ gallons; Clark and Veil, 2009)

CAPEX: Capital expenses (includes cost for materials, labor, and interest required to construct and begin operation)

${ }^{1}$ (Jackson and Myers, 2003)

${ }^{2}$ (ALL Consulting, 2012)

${ }^{3}$ (Fakhrul-Razi et al., 2009) 
treatment options can be refined by evaluating site-specific factors including water quality, site accessibility, cost of land, and cost of labor.

\section{Produced Water Management Plan and Application to Treatment System Design}

Management of produced water depends on several site-specific factors including produced water quantity and quality, local water needs, and treatment system costs (Figure 70). Constituents of concern limiting beneficial use can be targeted for treatment using a non-passive, passive, or hybrid approach. The non-passive approach can be used to treat COCs that may not be effectively removed with CWTSs (e.g. dissolved salts, boron, etc.) through the use of physical separation technologies (e.g. reverse osmosis, ion-exchange, filtration, etc.). The passive approach can be used to treat COCs that are effectively removed with CWTSs (e.g. metals, metalloids, oil and grease, nitrogenous compounds). A hybrid approach to designing CWTSs consisting of non-passive and passive approaches can be used to remove separable COCs including dissolved salts from produced water prior to introduction in wetland cells to expand treatability and beneficial use options and also lower capital and operational costs.

Because composition of produced waters typically varies from source to source (Tables 48), site-specific produced water characterization through sample collection and analysis is necessary to determine composition of the water to be treated. Constituents to be analyzed must include those that may hinder ability of the water to meet beneficial use criteria (Tables 9 and 10). After composition of produced water from the site is determined, COCs) can be identified by comparing constituent concentrations with beneficial use criteria that have been selected based on specific needs of the site location. Constituents that exceed the criteria concentrations are identified as COCs and will require treatment in order for the water to be suitable for use. With proper identification of COCs and design of CWTSs, waters ranging from fresh to hypersaline can be treated (e.g. Johnson et al., 2008b; Kanagy et al., 2008b).

A constructed wetland treatment system should be designed to promote specific biogeochemical pathways by which the COCs can be treated. Pilot-scale CWTS experiments performed during Phase II demonstrated successful design strategies for treating a variety of COCs including divalent metals, metalloids, oil and grease, and ammonia (Figures 2-5). Targeted pathways for treatment of COCs in the pilot-scale CWTS experiments included divalent metal sulfide precipitation through dissimilatory sulfate reduction, metal precipitation through oxidation, reduction of selenite to insoluble elemental selenium, aerobic biodegradation of oil, nitrification of ammonia to nitrate, denitrification of nitrate to nitrogen gas, separation of oil using an oil-water separator, and sorption of ammonia to zeolite.

CWTSs should be designed and built to promote specific environmental and geochemical conditions (Tables 20-23, 58, 61, and 62) in order for the targeted pathways to operate. For example, a CWTS designed to nitrify ammonia to nitrate must provide oxidizing conditions ( $\mathrm{DO}>2 \mathrm{mg} / \mathrm{L}$ ), neutral to slightly basic $\mathrm{pH}$ (6.6-9.7), and sufficient alkalinity $\left(>100 \mathrm{mg} / \mathrm{L}\right.$ as $\left.\mathrm{CaCO}_{3}\right)$ in order for nitrification to occur. Pilot-scale experiments achieved 
performance when the targeted environmental and geochemical conditions were met (Tables 26-50).

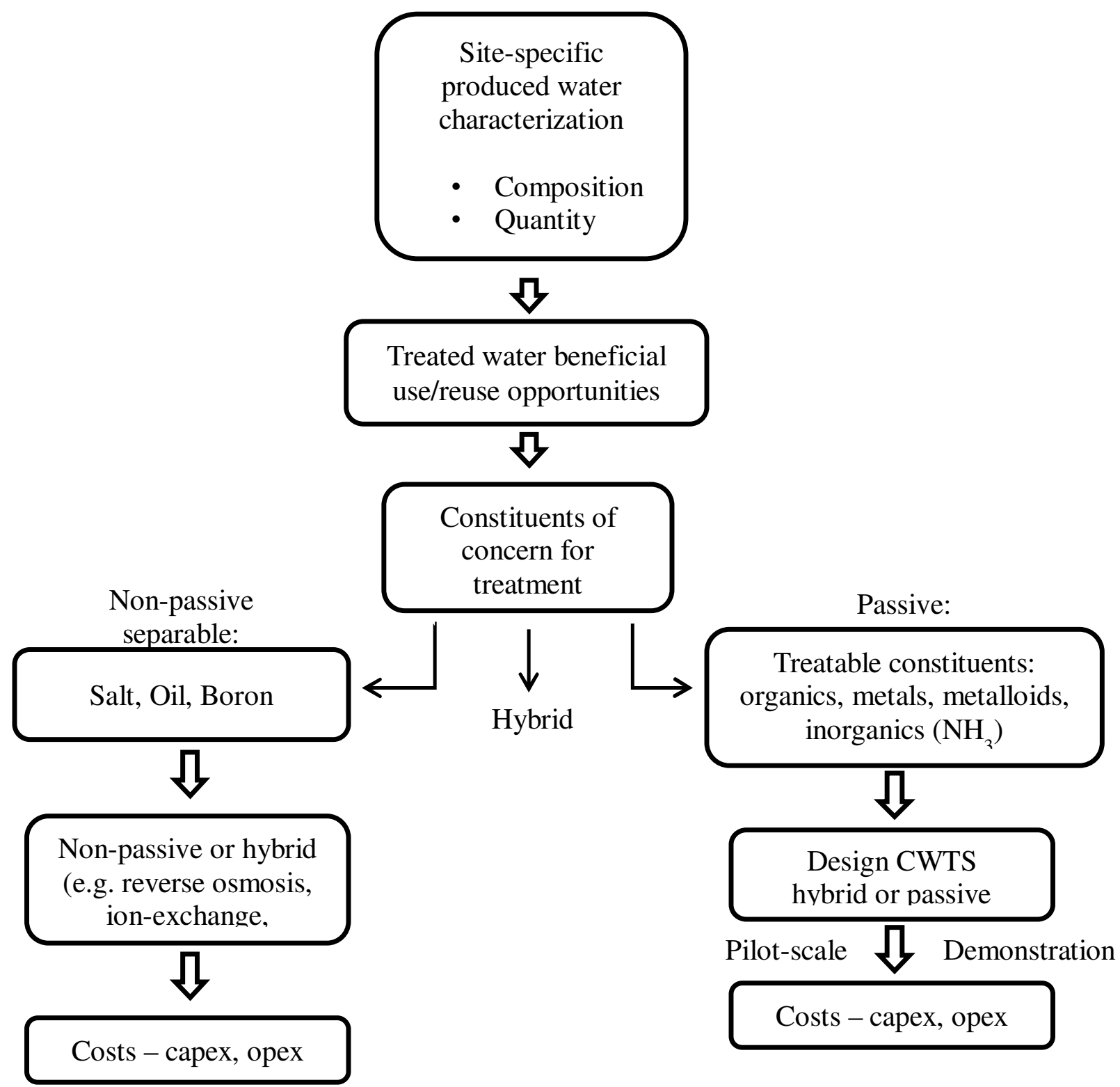

Figure 70. Produced water management plan, which is based in part on the methods used in Phases I-III and is intended for management of a site-specific produced water. 
Design strategies used in the pilot-scale CWTSs and demonstration CWTS to promote specific conditions that can be applied to design of full-scale CWTSs include plant and soil selection, water-depth selection, addition of amendments, and hydraulic retention time (HRT). These strategies allow conditions within the CWTS to be modified to achieve ranges necessary for the preferred biogeochemical pathways. For example, results of this project show that an oxidizing CWTS can be constructed with Typha latifolia, sandy soil, and a water depth of $33 \mathrm{~cm}$ or less. A reducing CWTS can be constructed with Schoenoplectus californicus, organic-rich soil, and a water depth of $46 \mathrm{~cm}$ or greater. to promote reduction reactions such as dissimilatory selenium reduction, AquaSmart ${ }^{\mathrm{TM}}$, an organic-rich nutrient amendment containing yeast, was added to some of the pilot-scale CWTSs and to the demonstration system. Crushed oyster shells were added to some pilotscale cells to increase alkalinity and raise $\mathrm{pH}$. An oil-water separator can be included in a CWTS to decrease oil and grease concentrations (Table 64, Figures 51 and 52), and zeolite minerals can be added to increase sorption of ammonia (Figure 30).

In the case of renovating a produced water containing COCs that require different biogeochemical pathways for treatment, a full-scale CWTS can be designed with sequential cells that promote different conditions. For instance, the pilot-scale CWTS for post-RO produced water was designed to promote oxidizing conditions within the first cell for nitrification of ammonia, while the subsequent three cells were designed to promote reducing conditions for denitrification of nitrate (Figure 2). By separating a CWTS into multiple cells, the conditions within each cell can be modified for removal of a variety of COCs. In addition, a CWTS designed with multiple cells allows for convenient sample collection points so that removal rate coefficients can be calculated and performance of individual cells can be evaluated.

By rearranging the integrated first-order rate coefficient equation (Equation 11), removal rate coefficients determined from the pilot-scale CWTS experiments (Tables 51-52, 54-56, 66-67) and confirmed by the demonstration system can be used to predict HRTs required to treat COCs in full-scale CWTSs:

$$
\text { Required Treatment HRT }(t)=\frac{-\ln \left([C] /\left[C_{0}\right]\right)}{k} \quad \text { Eqn. } 12
$$

where $\left[C_{\mathrm{o}}\right]$ is the concentration $(\mathrm{mg} / \mathrm{L})$ of a COC in the inflow, $[C]$ is the targeted treatment concentration $(\mathrm{mg} / \mathrm{L}), k$ is the first-order rate coefficient $\left(\mathrm{d}^{-1}\right)$, and $t$ is the time (days) and corresponds to HRT of the system.

The HRT calculated using Equation 12 can then be used to determine the surface area or "footprint" of a full-size CWTS for a given inflow rate:

$$
\text { Wetland Surface Area }(A)=\frac{Q \times t}{d} \quad \text { Eqn. } 13
$$

where $A$ is the required surface area of the CWTS $\left(\mathrm{m}^{2}\right), \mathrm{Q}$ is the inflow rate $\left(\mathrm{m}^{3} / \mathrm{d}\right), \mathrm{t}$ is the required HRT (days), and $\mathrm{d}$ is the water depth (m). 
Technology Transfer (Task 10)

The following is a list of journal papers and presentations for technology transfer of results from this project:

Alley, B., Beebe, A., Rodgers, J. H., Jr., and Castle, J. W., "A Comparative Characterization of Produced Water from Conventional and Unconventional Fossil Fuel Resources," American Association of Petroleum Geologists, Program and Abstracts, April 2010.

Alley, B., Beebe, A., Rodgers, J. H., Jr., and Castle, J. W., "Chemical and Physical Characterization of Produced Waters from Conventional and Unconventional Fossil Fuel Resources," Chemosphere, v. 85, p. 74-82. 2011.

Alley, B., Beebe, D. A., Rodgers, J. H., Jr., and Castle, J. W., "Chemical and Physical Characterization of Produced Waters from Conventional and Unconventional Fossil Fuel Resources," Clemson University $19^{\text {th }}$ Annual Hydrogeology Symposium Abstracts with Program, April 2011.

Alley, B. L., Rodgers, J. H., Jr., and Castle, J. W., "Performance of Constructed Wetland Treatment Systems Designed to Renovate Fresh Oilfield Produced Waters for Beneficial Uses," Geological Society of America Abstracts with Programs, v. 43, no. 5, p. 579, October 2011.

Alley, B. L., Rodgers, J. H., Jr., and Castle, J. W., "Renovating Fresh Oilfield Produced Waters for Beneficial Uses: Managing Constructed Wetland Treatment Systems for Performance," Society of Environmental Toxicology and Chemistry (SETAC) North America $32^{\text {nd }}$ Annual Meeting Abstract Book, p. 380, November 2011.

Alley, B. L., Willis, B., Rodgers, J., Jr., and Castle, J. W., "Seasonal Performance of a Hybrid Pilot-Scale Constructed Wetland Treatment System for Simulated Fresh Oil Field-Produced Water," Water, Air, \& Soil Pollution, v. 244. DOI 10.1007/s11270013-1639-5. 2013.

Alley, B. L., Willis, B., Rodgers, J. H., Jr., and Castle, J. W., "Water Depth and Treatment Performance of Free Water Surface Constructed Wetland Treatment Systems for Simulated Fresh Oilfield Produced Water," Clemson University $20^{\text {th }}$ Annual Hydrogeology Symposium Abstracts with Program, p. 2, April 2012.

Alley, B. L., Rodgers, J. H., Jr., and Castle, J. W., "Seasonal Performance of a Hybrid Pilot-Scale Constructed Wetland Treatment System for Simulated Fresh Oilfield Produced Water," Clemson University $21^{\text {st }}$ Annual Hydrogeology Symposium Abstracts with Program, p. 3, April 2013.

Beebe, D. A., Castle, J. W., and Rodgers, J. H., Jr., "Clinoptilolite as a Dual Purpose Sorbent Carrier in Constructed Wetland Treatment Systems Designed to Remove Ammonia," Clemson University $19^{\text {th }}$ Annual Hydrogeology Symposium Abstracts with Program, April 2011. 
Beebe, D. A., Song, Y., Castle, J. W., and Rodgers, J. H., Jr., "Pilot Study of Constructed Wetland Treatment Systems Designed for Removal of Ammonia in Oilfield Produced Water," Geological Society of America Abstracts with Programs, v. 43, no. 5, p. 580, October 2011.

Beebe, D. A., Song, Y., Castle, J. W., and Rodgers, J. H., Jr., "Designing Constructed Wetland Treatments Systems for Ammonia in Produced Water," Society of Environmental Toxicology and Chemistry (SETAC) North America $32^{\text {nd }}$ Annual Meeting Abstract Book, p. 377, November 2011.

Beebe, D. A., Alley, B., Castle, J. W., and Rodgers, J. H., Jr., "Potential for Beneficial Use of Coal-Bed Methane Produced Water in Western Alabama to Augment Water Supplies during Intense Drought," Geological Society of America Abstracts with Programs, v. 44, no. 4, p. 66, April 2012.

Beebe, D. A., Alley, B., Castle, J. W., and Rodgers, J. H., Jr., "Evaluation of Coal-Bed Methane Produced Water in Western Alabama for Use as a Water Resource during Drought," Clemson University $20^{\text {th }}$ Annual Hydrogeology Symposium Abstracts with Program, p. 4, April 2012.

Beebe, D. A., Castle, J. W., and Rodgers, J. H., Jr., "Experimental Studies of Evapotranspiration in Small Stand Wetlands," Geological Society of America Abstracts with Programs, v. 44, no. 7, p. 581, November 2012.

Beebe, D. A., Castle, J. W., and Rodgers, J. H., Jr., "Effects of Evapotranspiration on Water Treatment Performance in Constructed Wetlands," Clemson University $21^{\text {st }}$ Annual Hydrogeology Symposium Abstracts with Program, p. 5, April 2013.

Castle, J. W., Rodgers, J. H., Jr., Alley, B., Spacil, M., Beebe, A., Pardue, M., and Song, Y., "Biogeochemical Processes for Treating Oil and Gas Produced Waters Using Hybrid Constructed Wetland Treatment Systems," American Association of Petroleum Geologists, Program and Abstracts, April 2010.

Castle, J. W., Rodgers, J. H., Jr., Spacil, M., Alley, B., Horner, J. E, and Pardue, M., "Pilot-Scale Constructed Wetland Treatment Systems for Oil \& Gas Produced Waters," $17^{\text {th }}$ Annual International Petroleum and Biofuels Environmental Conference Program and Abstracts, p. 47, September 2010.

Castle, J. W., Rodgers, J. H., Jr., Spacil, M., Horner, J. E, Alley, B., and Pardue, M., "Pilot-Scale Constructed Wetland Systems for Treating Energy-Produced Waters," Ground Water Protection Council Annual Forum, Water \& Energy in Changing Climates, Pittsburgh, PA, September 29, 2010.

Castle, J. W., Rodgers, J. H., Jr., Spacil, M., Alley, B., and Pardue, M., “A Pilot-Scale Study to Apply Biogeochemical Processes of Natural Wetlands to Treating Impaired Waters Using Constructed Wetland Treatment Systems," Geological Society of America Abstracts with Programs, v. 42, no. 5, p. 640, November 2010.

Castle, J. W., Rodgers, J. H., Jr., Spacil, M. M., and Ritter, C. L., “Treating Selenium 
in Energy-derived Produced Waters Using Constructed Wetland Treatment Systems," Geological Society of America Abstracts with Programs, v. 43, no. 5, p. 578, October 2011.

Coffey, R. E., Castle, J. W., and Rodgers, J. H., Jr., "A Demonstration Constructed Wetland Treatment System for Unconventional Gas Produced Water," Clemson University $21^{\text {st }}$ Annual Hydrogeology Symposium Abstracts with Program, p. 10, April 2013.

Jurinko, K. N., Ritter, C. L., Castle, J. W., and Rodgers, J. H., Jr., "Biogeochemical Process in a Pilot-Scale Constructed Wetland Treatment System Designed to Remove Metals from Produced Water," Clemson University $19^{\text {th }}$ Annual Hydrogeology Symposium Abstracts with Program, April 2011.

Pardue, M. J., Castle, J. W., and Rodgers, J. H., Jr., "Evaluation of a Pilot-Scale Constructed Wetland System for Treatment of a Specific Oilfield Produced Water," Clemson University $19^{\text {th }}$ Annual Hydrogeology Symposium Abstracts with Program, April 2011.

Pardue, M. J., Castle, J. W., and Rodgers, J. H., Jr., "A Biogeochemical Approach to Renovation of Energy-Derived Produced Water Using a Pilot-Scale Constructed Wetland Treatment System," Geological Society of America Abstracts with Programs, v. 43, no. 5, p. 580, October 2011.

Pardue, M. J., Castle, J. W., Huddleston, G. M., and Rodgers, J. H., Jr., "Treatment of Oilfield Produced Water Using a Pilot-Scale Constructed Wetland Treatment System," Clemson University $20^{\text {th }}$ Annual Hydrogeology Symposium Abstracts with Program, p. 33, April 2012.

Ritter, C. L., Jurinko, K. N., Castle, J. W., and Rodgers, J. H., Jr., "Biogeochemical Processes in a Constructed Wetland Treatment System Designed for Removal of Selenium from Energy-Derived Water," Clemson University $19^{\text {th }}$ Annual Hydrogeology Symposium Abstracts with Program, April 2011.

Rodgers, J. H., Jr., Castle, J. W., Horner, J. E, Spacil, M., Eggert, D., Alley, B., Beebe, A., Pham, M. P. T., Song, Y., Myers, J. E., Murray Gulde, C., Huddleston, M., and Mooney, D., "Constructed Wetland Treatment Systems for Renovation of Oilfield Produced Water for Beneficial Use," Clemson University $17^{\text {th }}$ Annual Hydrogeology Symposium Abstracts with Program, April 2009.

Rodgers, J. H., Jr., and Castle, J. W., "Characteristics of Produced Waters and Biogeochemical Processes for Effective Management Using Constructed Wetland Treatment Systems," Goldschmidt International Conference on Earth, Energy, and the Environment, Geochimica et Cosmochimica Acta, v. 74, issue 12, Supplement 1, p. A876, June 2010.

Rodgers, J. H., Jr., Castle, J. W., Spacil, M. M., and Ritter, C., "Constructed Wetland Treatment Systems for Energy-derived Produced Waters: Treating Selenium for Surface Water Discharge," Society of Environmental Toxicology and Chemistry 
(SETAC) North America $32^{\text {nd }}$ Annual Meeting Abstract Book, p. 376, November 2011.

Spacil, M. M., Rodgers, J. H., Jr., Castle, J. W., and Chao, W. Y., "Performance of a Pilot-Scale Constructed Wetland Treatment System for Selenium, Arsenic, and Low Molecular Weight Organics in Simulated Fresh Produced Water," Clemson University $19^{\text {th }}$ Annual Hydrogeology Symposium Abstracts with Program, April 2011.

Spacil, M. M., Rodgers, J. H., Jr., Castle, J. W., and Chao, W. Y., "Performance of a Pilot-Scale Constructed Wetland Treatment System for Selenium, Arsenic, and Low Molecular Weight Organics in Simulated Fresh Produced Water," Environmental Geosciences, v. 18, p. 145-156. 2011.

Spacil, M. M., Rodgers, J. H., Jr., Castle, J. W., and Chao, W. Y., "Treatment of Selenium in Produced Water Using a Pilot-scale Constructed Wetland Treatment System," Society of Environmental Toxicology and Chemistry (SETAC) North America 33 ${ }^{\text {rd }}$ Annual Meeting Abstract Book, p. 273, November 2012.

\section{CONCLUSION}

A wide range of chemical, physical, and risk characteristics was present in the produced waters investigated. Constituents of concern identified as requiring treatment in produced waters prior to reuse or discharge include dissolved salts, metals, metalloids, organics, and post-RO constituents. Dissolved salts, expressed as salinity, conductivity, or TDS in published reports and in data provided by industry, occur over a wide range in produced waters. Cations found in produced water commonly include sodium, calcium, magnesium, and potassium; anions present often include chloride, sulfate and bicarbonate. Reverse osmosis (RO) is sometimes used to reduce the concentration of cations and anions in produced waters. However, specific constituents such as ammonia can pass through RO membranes and require further treatment. Metals that are of concern in produced waters can include any or all of the following: iron, manganese, zinc, cadmium, cobalt, copper, lead, mercury, chromium, nickel, and zinc. Based on our analysis, other constituents of concern identified in produced waters include arsenic and selenium. Produced waters can contain a range of organic constituents from oil and grease to low molecular-weight aromatic hydrocarbons such as benzene, toluene, ethylbenzene, and xylene (BTEX).

Pilot-scale constructed wetland treatment systems were designed and constructed at Clemson University based on fundamental reactions and processes needed to treat (transfer and transform) targeted constituents in produced waters. Separate systems were constructed for representative constituents with specific water compositions selected based on results of the previous tasks. We investigated four waters comparing a range of characteristics or constituents of concern for treatment (metals, metalloids, oil and grease, and ammonia). The pilot-scale systems were designed to produce biogeochemical conditions that result in targeted treatment pathways for constituents that occur in produced waters. Systems were constructed so that the sequence of biogeochemical 
conditions can be changed as needed to achieve the most effective treatment.

Performance measurements from the pilot-scale constructed wetland systems indicated effective treatment of targeted constituents in the four types of produced waters investigated. Data from the pilot-scale phase of the project were used to design a demonstration system. In collaboration with Chevron, a site for constructing the demonstration system was selected near Berry, Alabama. This site is within a producing coalbed methane field. The demonstration CWTS was designed and built to promote specific environmental and geochemical conditions in order for targeted treatment pathways to operate.

Specific experiments in support of designing a demonstration system were performed to gain a better understanding of conditions affecting treatment pathways and hybridized design strategies that can be incorporated into constructed wetland treatment, including the demonstration CWTS. The experiments included investigation of a zeolite for sorption of ammonia, the effect of water depth and seasonal variation on conditions and treatment, effect of adding a nutrient amendment, incorporation of an oil/water separator, and evapotranspiration.

Experiments were designed to compare seedling growth of corn (Zea maya), cabbage (Brassica oleracea), and soybean (Phaseolus vulgaris) between untreated and treated simulated produced water. The difference in early seedling growth between the waters was determined to be statistically significant, with higher growth rate in the treated water.

Toxicity tests using Ceriodaphnia dubia and Pimephales promelas compared the potential between untreated and treated simulated fresh produced water to cause adverse effects to receiving system biota. Statistical analysis of the experimental data indicated a significant difference in the response of $C$. dubia and P. promelas between untreated and treated simulated fresh produced water, with greater survival in the treated water.

Evapotranspiration data were collected. Theoretical lysimeter outflow determined using the Penman Monteith equation and mid-summer peak Typha latifolia (cattail) crop coefficient $\left(\mathrm{K}_{\mathrm{c}}\right)$ was compared with measured lysimeter outflow. The results demonstrate application of the Penman Monteith equation to estimating evapotranspiration in the pilot-scale CWTSs used in this investigation. Tracer tests indicate a significant difference in vertical transport of constituents caused by plant transpiration.

The influence of water depth on treatment performance of pilot-scale CWTSs for renovating simulated fresh oilfield produced water was investigated. Divalent metal concentrations were greater in outflow from wetland cells having 15 and $23 \mathrm{~cm}$ water depths than from cells having 46 and $56 \mathrm{~cm}$ water depths. The cells having 46 and $56 \mathrm{~cm}$ water depths provided reducing conditions conducive for dissimilatory sulfate reduction, which contributed to removal of divalent metals from the simulated produced water. Wetland cells with 15 and $23 \mathrm{~cm}$ water depths were more effective for removing oil from the simulated produced water than cells with greater water depths because shallower depth promoted conditions favorable for aerobic degradation of oil. 
Produced water samples were collected from the demonstration CWTS site in central Alabama and analyzed. Comparison with surface water quality criteria yielded the following COCs to be targeted for treatment: ammonia, barium, cadmium, iron, manganese, nitrate, and selenium. A demonstration CWTS was designed and constructed to promote the biogeochemical conditions required for effective treatment of identified COCs in the produced water. Oxidizing cells (targeted DO greater than $2.0 \mathrm{mg} / \mathrm{L}$ ) were included in each series to promote microbial nitrification of ammonia and precipitation of iron and manganese. Reducing cells (targeted DO less than $2.0 \mathrm{mg} / \mathrm{L}$ ) were included to promote microbial denitrification of nitrate, dissimilatory sulfate reduction and metal sulfide formation, and dissimilatory selenium reduction. Performance of the demonstration CWTS was monitored using sampling methods that allow for calculation of removal efficiency, removal rate, and removal extent of constituents of concern. The demonstration CWTS successfully achieved consistent treatment of COCs even as their concentrations in the produced water varied by orders of magnitude.

Design strategies used in the pilot-scale and demonstration CWTSs to promote specific conditions that can be applied to designing full-scale CWTSs include plant and soil selection, water-depth selection, addition of amendments, hydraulic retention time (HRT), and hybrid components. These strategies allow conditions within a CWTS to be modified to achieve ranges necessary for the preferred biogeochemical processes for treating fresh to saline produced waters. In the case of renovating a produced water containing COCs that require different biogeochemical pathways for treatment, a CWTS can be designed with sequential cells that promote different conditions. By incorporating multiple wetland cells in a CWTS, conditions within each cell can be modified for removal of specific COCs. In addition, a CWTS designed with multiple cells allows for convenient sample collection so that biogeochemical conditions of individual cells can be monitored and performance can be evaluated. Removal rate coefficients determined from the pilot-scale CWTS experiments and confirmed by the demonstration system can be used to calculate HRTs required to treat COCs in full-scale CWTSs. The calculated HRTs can then be used to determine the surface area or "footprint" of a full-size CWTS for a given inflow rate of produced water.

\section{REFERENCES CITED}

Abdollahi, H. and Nedwell, D.B. 1979. Seasonal Temperature as a Factor Influencing Bacterial Sulfate Reduction in a Saltmarsh Sediment. Microbial Ecology, 5: 73-79. Abollino, O., Aceto, M., Malandrino, M., Sarzanini, C., Mentasti, E. 2003. Adsorption of heavy metals on Na-montmorillonite. Effect of $\mathrm{pH}$ and organic substances, Water Research, 37, 1619-1627.

Agarwal, A., delos Angeles, M. S., Bhatia, R., Chéret, I., Davila-Poblete, S., Falkenmark, M., Villareal, M. F., Jønch-Clausen, T., Kadi, M. A., Kindler, J., Rees, J., Roberts, P., Rogers, P., Solanes, M., \& Wright, A. 2000. Integrated Water Resources Management. Global Water Partnership Technical Advisory Committee (TAC) No. 4. 
Aguirre, P., Ojeda, E., García, J., Barragán, J., Mujeriego, R., 2005. Effect of water depth on the removal of organic matter in horizontal subsurface flow constructed wetlands. Journal of Environmental Science and Health 40, 1457-1466.

ALL Consulting. 2003. "Handbook on CBM Produced Water: Management and Beneficial Use Alternatives," Prepared for Ground Water Protection Research Foundation, U.S. Department of Energy, National Petroleum Technology Office, and Bureau of Land Management.

ALL Consulting, 2012. Produced Water Treatment Technology Catalog and Decision Tool, Tulsa, Oklahoma. Available at: http://www.allllc.com/projects/produced_water_tool/index.php.

Allen, H.E., Hansen, D.J., 1996. The importance of trace metal speciation to water quality criteria. Water Environment Research 68, 42-54.

Allen, H.E., Fu, G., and Deng, B. 1993. Analysis of acid-volatile sulfide (avs) and simultaneously extracted metals (SEM) for the estimation of potential toxicity in aquatic sediments. Environmental Toxicology and Chemistry, 12:1441-1453.

Allen, R.G., Pereira, L.S., Raes, D., and M. Smith. 1998. Crop Evapotranspiration: Guidelines for Computing Crop Requirements. Irrigation and Drainage Paper No. 56, FAO, Rome, Italy.

Alley, B.L., Beebe, D.A., Rodgers, J.H. Jr., Castle, J.W., 2011. Chemical and physical characterization of produced water from conventional and unconventional fossil fuel resources. Chemosphere 85, 74-82.

American Petroleum Institute (API), 2004. Risk-based screening levels for the protection of livestock exposed to petroleum hydrocarbons. Publ. No. 4733. Washington DC.

American Public Health Association (APHA), 2005. American Water Works Association and Water Pollution Control Federation. Standard methods for examination of water and wastewater ( $21^{\text {st }}$ ed.). Washington, DC: American Public Health Association.

American Society of Testing and Materials (ASTM), 2011. Standard practices for sampling of waterborne oils. ASTM D4489-95.

Amor, Z., Bariou, B., Mameri, N., Taky, M., Nicolas, S., and Elmidaoui, A. 2001. Fluoride removal from brackish water by electrodialysis. Desalination 133: 215-223.

Armstrong, J., Afreen-Zobayed, F., Blyth, S., and Armstrong, W., 1999. Phragmites australis: Effects of shoot submergence on seedling growth and survival and radial oxygen loss from roots. Aquatic Botany, 64: 275-289.

Ayers, R.S., Westcot, D.W. 1994. Water quality for agriculture. FAO irrigation and drainage paper, vol. 29. Rome: FAO.

Barton, L. I., \& Tomei, F. A. (1995). In Barton L. L. (Ed.), Biotechnology handbook: Sulfate-reducing bacteria. New York: Plenum Press.

Beebe, D.A., B. Alley, J.W. Castle, and J.H. Rodgers, Jr. 2012. Potential for Beneficial Use of Coal-Bed Methane Produced Water in Western Alabama to Augment Water Supplies during Intense Drought. Geological Society of America Abstracts with Programs, v. 44, no. 4, p. 66.

Benko, K. and Drewes, J. E. 2008. Produced Water in the Western United States: Geographical Distribution, Occurrence, and Composition. Environmental Engineering Science, 25(2): 239-246. 
Bennion, D.B., Thomas, F.B., and Bietz, R.F. 1995. Low Permeability Gas Reservoirs: Problems, Opportunities and Solutions for Drilling, Completion, Stimulation and Production, Society of Petroleum Engineers, Inc., Prepared for presentation at the Gas Technology Conference held in Calgary, Alberta, Canada, April 28 - May 1 2006.

Bhamidimarri, R., Shilton, A., Armstrong, I., Jacobson, P., \& Scarlet, D. 1991. Constructed wetlands for wastewater treatment: the New Zealand experience. Water Science \& Technology, 24: 247-253.

Biesinger, K.E. and Christensen, G.M. 1972. Effects of various metals on survival, growth, reproduction, and metabolism of Daphnia magna. Journal of the Fisheries Research Board of Canada, 29(12): 1691-1700.

Brix, H. 1994. Functions of Macrophytes in Constructed Wetlands. Wat. Sci. Tech., 29(4):71-78.

Brix, H., Sorrell, B.K., 1996. Oxygen stress in wetland plants: comparison of deoxygenated and reducing root environments. Functional Ecology. 10, 521-526.

Brookins, D.G. 1988. Eh-pH Diagrams for Geochemistry. Springer, Berlin., Pp. 176.

Buhl, K. 1998. Toxicity of Proposed Water Quality Criteria-Based Mixtures of 11 Inorganics to Ceriodaphnia dubia and Fathead Minnow. U.S. Geological Survey, Environmental and Contaminants Research Center.

Burnett, D. 2007. Produced Water Reuse. Texas A\&M Petroleum Engineering.

Buttner, J., Soderberg, R.W., and Terlizzi, D.E. 1993. An Introduction to Water Chemistry in Freshwater Aquaculture. Northeastern Regional Aquaculture Center, University of Massachusetts Dartmouth.

Carbonell, A.A., J.D. Porthouse, C.K. Mulbah, R.D. DeLaune and W.H. Patrick Jr., 1999. Metal solubility in phosphogypsum-amended sediment under controlled $\mathrm{pH}$ and redox conditions. J. Environ. Qual., 28: 232-242.

Carlson A.R., Nelson H., and Hammermeister, D. 1986. Evaluation of site specific criteria for copper and zinc. EPA 600/3-86-026, United States Environmental Protection Agency, Duluth, IA.

Caselles-Osorio, A., García, J., 2006. Performance of experimental horizontal subsurface flow constructed wetlands fed with dissolved or particulate organic matter. Water Research 40, 3603-3611.

Clark, C.E., and Veil, J.A., 2009. Produced Water Volumes and Management Practices in the United States [http://www.osti.gov/bridge], prepared by the Environmental Science Division, Argonne National Laboratory for the U.S. Department of Energy, Office of Fossil Energy, National Energy Technology Laboratory.

Crowe, D. 2007. Membrane Technology for the Treatment of Produced Water. GeoPure Water Technologies.

DeGraeve, G.M., J.D. Cooney, B.H. Marsh, T.L. Pollock, and N.G. Reichenbach. 1992. variability in the performance of the 7-d Ceriodaphnia dubia survival and reproduction test: an intra- and interlaboratory study. Environ. Toxicol. Chem. 11(6): 851-866.

Delaune, R.D., Hambrick, G.A. III, Patrick, W.H. Jr., 1980. Degradation of hydrocarbons in oxidized and reduced sediments. Marine Pollution Bulletin. Pergamon Press Ltd. 11, 103-106.

de Souza, M. P., Chu, D., Zhao, M., Zayed, A. M., Ruzin, S. E., Schichnes, D., et al. 
(1999). Rhizosphere Bacteria Enhance Selenium Accumulation and Volatilization by Indian Mustard. Plant Physiology , 119, 565-573.

DiFilippo, M.N. 2004. Use of Produced Water in Recirculating Cooling Systems at Power Generating Facilities.DOE Semi-Annual Technical Progress Report.

Dodds, P.C. 2008. Marcellus Shale also has its risk. Morgantown Dominion Post.

Dorman, L., Rodgers, J.H., Jr., Castle, J.W. 2010. Characterization of Ash-Basin Waters from a Risk-Based Perspective. Water, Air, and Soil Pollution. 206:175-185.

Edwards, E.A. and Grbic-Galic, D. 1992. Complete mineralization of benzene by aquifer microorganisms under strictly anaerobic conditions. Appl. Environ. Microbiol., 58: 2663-2666.

Edwards, M. 1994. Chemistry of arsenic removal during coagulation and Fe-Mn oxidation. J. Am. Water Works Assoc., 86: 64-78.

Eggert, D.A, J. H. Rodgers, Jr., G. M. Huddleston, and C. E. Hensman. 2008. Performance of Pilot-Scale Constructed Wetland Treatment Systems for Flue Gas Desulfurization (FGD) Waters. Environmental Geosciences, 19: 11-19.

EIA. 1995. Coal Data: A Reference. Energy Information Administration. DOE/EIA0064(93).

EIA. 2008. Annual Energy Outlook 2008: With Projections to 2030., DOE/EIA0383(2008) Washington, D.C.

ESN Rocky Mountain. 2003. Produced Gas and Water Testing of CBM Gas Wells in the Raton Basin. Report Prepared by Rocky Mountain Environmental Services Network, Denver Colorado for the COGCC.

European Union, 1998. Council Directive 98/83/EC of 3 November 1998 on the quality of water intended for human consumption. Official Journal, L330/45, 05/12/1998.

Faulkner, S.P., W.H. Patrick, Jr., R.P. Gambrell, 1989. Field techniques for measuring wetland soil parameters. Soil Sci. Soc. Am. J. 53, 883-890.

Fakhrul-Razi, A., A. Pendashteh, L. Abdullah, D. Biak, S. Madaeni, and Z. Abidin, 2009. Review of technologies for oil and gas produced water treatment. Journal of Hazardous Materials, 170: 530-551.

Fieser, A.H., Sykora, J.L., Kostalos, M.S., Wu, Y.C. and Weyel, D.W., 1986. Effect of fluorides on survival and reproduction of Daphnia magna. J. Water Pollut. Control Fed. 58: 82-86.

Fillo, J.P. and Evans, J.M. 1990. Characterization and management of produced waters from underground natural gas storage reservoirs. American Gas Association Operation Section Proceedings, pp. 448-459.

Fillo, J.P., Koraido, S.M., and Evans, J.M. 1992. Sources, Characteristics, and Management of Produced Waters from Natural Gas Production and Storage Operations. Produced Water: Technological/Environmental Issues and Solutions. J.P. Ray, F.R. Engelhardt eds. pp. 73-88.

Ford, D.L. 1998. Introduction to toxicity identification and toxicity reduction evaluations. In $\left(2^{\text {nd }}\right.$ Eds.) Toxicity reduction: evaluation and control. Technomic Publishing Company, Inc., Lancaster, PA, Pp. 17-28.

Frankenberger, W.T. and Engberg, R.A. 1998. Environmental Chemistry of Selenium, Volume 64 of Books in soils, plants, and the environment. pp. 713.

Gambrell R.P. and W.H.Patrick, Jr., 1978, Chemical and Microbiological Properties of Anaerobic Soils and Sediments, Ann Arbor Pub., Ann Arbor, pp. 390. 
Garbisu, C., Ishii, T., Leighton, T., Buchanan, B. B. 1996. Bacterial reduction of selenite to elemental selenium. Chemical Geology, 132: 199-204.

Gerardi, Michael H., 2007. Oxidation-Reduction Potential in Waste Water Treatment, Interstate Water Report; NEIWPCC, Winter, 2007.

Gillespie Jr. WB, Hawkins WB, Rodgers Jr. JH, Cano ML, Dorn PB. 1999. Transfers and transformations of zinc in flowthrough wetland cells. Ecotoxicol Environ Saf., 43:126-32.

Gillespie, W.B. Jr., Hawkins, W.B., Rodgers, J.H. Jr., Cano, M.L., and Dorn, P.B. 2000. Transfers and transformations of zinc in constructed wetlands: Mitigation of a refinery effluent. Ecological Engineering, 14: 279-292.

GPRI. 2003. Conversion of Oil Field Produced Brine to Fresh Water. Global Petroleum Research Institute Designs.

Gujer W. and D. Jenkins, 1974. A Nitrification model for contact stabilization activated sludge process. Water Research, 9(5): 5.

Halda-Alija, L. and Johnston, T.C. 1999. Diversity of culturable heterotrophic aerobic bacteria in pristine stream bed sediments. Canadian Journal of Microbiology, 45: 879-884.

Halverson, N. V. 2004. Review of Constructed Subsurface Flow vs. Surface Flow Wetlands. Westinghouse Savannah River Company, Aiken, SC. Prepared for the U.S. Dept. of Energy.

Hawkins, W.B., Rodgers, J.H. Jr., Gillespie, W.B. Jr., Dunn, A.W., Dorn, P.B., and Cano, M.L. 1997. Design and Construction of Wetlands for Aqueous Transfers and Transformations of Selected Metals. Ecotoxicology and Environmental Safety, 36: 238-248.

Hecky, R.E. and Kilham, P. 1973. Diatoms in Alkaline, Saline Lakes: Ecology and Geochemical Implications. Limnology and Oceanography, 18(1): 53-71.

Hering, J. G., Chen, P. Y., Wilkie, J. A., Elimelech, M. 1996. Arsenic removal by ferric chloride. J. Am. Water Works Assoc. 88, 155-167.

Hill, D.G., Gas Storage Characteristics of Fracture Shale Plays, presented at the Strategic Research Institute Gas Shale Conference, June 11-12, 2002, Denver, Colorado.

Hill, D.G., Lombardi, T.E., and Martin, J.P. 2008. Fractured Shale Gas Potential in New York. Texas A\&M Petroleum Engineering.

Horner, J.E., Castle, J.W., Rodgers, J.H. Jr., Murray-Gulde, C., and Myers, J.E. 2012. Design and performance of pilot-scale constructed wetland treatment systems for treating oilfield produced water from Sub-Saharan Africa. Water Air Soil Pollution, 223: 1945-1957.

Huddleston, G.M. III, Rodgers, J.H. Jr. 2008.Design of a constructed wetland for treatment of copper-contaminated wastewater. Environmental Geosciences 15: 9-19.

Huddleston, G.M. III, Gillespie, W.B., Rodgers, J.H. Jr. 2000. Using constructed wetlands to treat biochemical oxygen demand and ammonia associated with a refinery effluent. Ecotoxicol. Environ. Saf., 45: 188-193.

Hughes, D. G., McKenzie, P., 1975. The microbial degradation of oil in the sea. P. Proc. R. Soc. 189, 375-390.

Hunter, J. A., and Moser, P. H., 1990, Ground water availability in Jefferson County, Alabama: Alabama Geological Survey Special Map 224, 68 p.

Hutson, S.S., N.L. Barber, J.F. Kenny, K.S. Linsey, D.S. Lumia, and M.A. Maupin, 2004, 
"Estimated Use of Water in the United States in 2000," U.S. Geological Survey Circular 1268, pp. 46.

Ike, M., Takahashi, K., Fujita, T., Kashiwa, M., Fujita, M. 2000. Selenate reduction by bacteria isolated from aquatic environment free from selenium contamination. Water Resources, 34(11): 3019-3025.

Jackson, L.M., and J.E. Myers. Design and construction of pilot wetlands for producedwater treatment. SPE Annual Technical Conference and Exhibition, SPE 84587 2003.

John, D. A. \& Leventhal, J. S. 1995. Bioavailability of Metals. Ch. 2 in: Preliminary compilation of descriptive geoenvironmental mineral deposit models Edward A. du Bray, Editor. U.S. Department of the Interior U.S. Geological Survey Open-File Report 95-831, Denver, Colorado.

Johnson, B., Kanagy, L., Rodgers, J.H., Jr., and Castle, J.W. 2008a. Chemical, Physical, and Risk Characterization of Natural Gas Storage Produced Water. Water, Air, and Soil Pollution, 191: 33-54.

Johnson, B.M., Kanagy, L.E., Rodgers, J.H. Jr., and Castle, J.W. 2008b. Feasibility of a pilot-scale hybrid constructed wetland treatment system for simulated natural gas storage produced waters. Environmental Geosciences, 15(3): 91-104.

Josselyn, M. N., Faulkner, S. P. and Patrick,W. H. 1990. Relationships between seasonally wet soils and occurrence of wetland plants in California, Wetlands 10(1): 7-26.

Junfeng, D., Xiang, L. and Zhifeng, H. 2007. Anaerobic BTEX degradation in soil bioaugmented with mixed consortia under nitrate reducing conditions. Journal of Environmental Sciences, 20: 585-592.

Kadlec, R.H. 1999. Chemical, Physical and Biological Cycles in Treatment Wetlands. Water Science and Technology. 40(3): 37-44.

Kadlec, R.H., S.D. Wallace, 2009. Treatment Wetlands, 2nd Edition. Boca Raton, CRC Press.

Kalff, J. 2002. Limnology. Upper Saddle River, N.J., Prentice Hall.

Kamal, M., Ghaly, A.E., Mahmoud, N., and Côtè, R. 2004. Phytoaccumulation of heavy metals by aquatic plants. Environment International 29, 1029-1039.

Kanagy, L. E., Johnson, B. M., Castle, J. W., and Rodgers, J. H. Jr. (2008a). Hydrosoil conditions in a pilot-scale constructed wetland treatment system for natural gas storage produced waters. Environmental Geosciences 15(3): 105-113.

Kanagy, L. E., Johnson, B. M., Castle, J. W., and Rodgers, J. H. Jr. (2008b). Design and performance of a pilot-scale constructed wetland treatment system for natural gas storage produced water. Bioresource Technology 99: 1877-1885.

Knight, R. L., R. H. Kadlec, and H. M. Ohlendorf. 1999. The use of treatment wetlands for petroleum industry effluents. Environ. Sci. Technol., 33: 973-980.

Knowles, 1981. R. Knowles, Denitrification. Ecological Bulletin, Stockholm 33: 315-329.

Korda, A., Santas, P., Tenente, A., Santas, R. 1997. Petroleum hydrocarbon bioremediation: sampling and analytical techniques, in situ treatments and commercial microorganisms currently used. Applied Microbiology Biotechnology 48, 677-686

Kuuskraa, V.A. and Ammer, J. 2004. Tight Gas Sands Development-How to Dramatically Improve Recovery Efficiency, Unconventional Resources, GasTIPS, Winter 2004.

Lasier, P.J., Winger, P.V., and Bogenrieder, K.J. 1999. Toxicity of Manganese to 
Ceriodaphnia dubia and Hyalella azteca. Arch. Environ. Contam. Toxicol. 38: 298304.

LeBlanc, G.A. 1980. Acute toxicity of priority pollutants to water flea (Daphnia magna). Bulletin of Environmental Contamination and Toxicology, 24: 684-691.

Li, N., Lee, H. K. 2001. Solid-phase extraction of polycyclic aromatic hydrocarbons in surMIce water: negative effect of humic acid. Journal of Chromatography A. 921, 255-263.

Liske, B., 2005, "Recovery of More Oil-in-Place at Lower Production Costs While Creating a Beneficial Water Resource," presented at the DOE/PERF Water Program Review, Annapolis, MD, Nov. 1-4.

Lortie, L., Gould, W. D., Rajan, S., McCready, R. G. L., Cheng, K.-J. 1992. Reduction of selenate and selenite to elemental selenium by a Pseudomonas stutzeri isolate. Applied and Environmental Microbiology, 58(12): 4042-4044.

Lovley, D.R. 1997. Potential for anaerobic bioremediation of BTEX in petroleumcontaminated aquifers. Journal of Industrial Microbiology and Biotechnology, 18: 75-81.

Lovley, D.R., Coates, J.D., Woodward, J.C., and Phillips, E.J.P. 1995. Benzene oxidation coupled to sulfate reduction. Applied and Environmental Microbiology, 61: 953958.

Lyman, J. and Fleming, R.H. 1940. Composition of sea water. J. Mar. Res. 3, 134.

Maiers, D.T., Wichlacz, P. L., Thompson, D.L., \& Bruhn, D.F. 1988. Selenate reduction by bacteria from a selenium-rich environment. Applied and Environmental Microbiology, 54(10): 2591-2593.

Martin, J.F., E. Hofherr, and M. Quigley, 2003. Effects of Typha latifolia transpiration and harvesting on nitrate concentrations in surface water of wetland microcosms. Wetlands, 23(4): 835-844.

Massachusetts Department of Environmental Protection (MDEP). 2009. Method for the determination of air-phase petroleum hydrocarbons (APH). Office of Research and Standards, Bureau of Waste Site Cleanup Final Revision 1.

McIntosh, J.C., Walter, L.M. and Martini, A.M. 2002. Pleistocene recharge to midcontinent basins: Effects on salinity structure and microbial gas generation. Geochimica Acta, 66(10): 1681-1700.

Montana GWIC. 2009. Coal Bed Methane Powder River Basin: Montana Bureau of Mines and Geology Groundwater Information Center.

Mooney F.D., and Murray-Gulde, C.L. 2008. Constructed treatment wetlands for flue gas desulfurization waters: full-scale design, construction issues, and performance. Environmental Geosciences 15: 131-141.

Moret, S., Piani, B., Bortolomeazzi, R., Contel, L. 1997. HPLC determination of polycyclic aromatic hydrocarbons in olive oils. Z Lebensm Unters Forsch A. 205, 116-120.

Morse, J.W., Presley, B.J., Taylor, R.J., Benoit, G., and Santschi P. 1993. Trace metal chemistry of Galveston Bay: Water, sediments and biota. Mar. Environ. Res. 36: 137.

Moshiri, G. A. 1993. Constructed Wetlands for Water Quality Improvement, (Eds.) Lewis Publishers, Boca Raton, FL, Pp. 632.

Murray-Gulde, C.L., Bearr, J., and Rodgers, J.H. Jr. 2005a. Evaluation of a constructed 
wetland treatment system specifically designed to decrease bioavailable copper in a wastestream. Ecotoxicology and Environmental Safety, 61: 60-73.

Murray-Gulde, C.L., Huddelston, G.M. III, Garber, K.V., and Rodgers, J.H. Jr. 2005b. Contributions of Schenoplectus californicus in a constructed wetland system receiving copper contaminated wastewater. Water Air and Soil, 163: 355-378.

Murray-Gulde, C.L., Heatly, J.E., Karanfil, T., Rodgers, J.H. Jr., and Myers, J.E. 2003. Performance of a hybrid reverse osmosis-constructed wetland treatment system for brackish water. Water Research, 37: 705-713.

Nelson, C.R., 1999, Changing perceptions regarding the size and production potential of coalbed methane resources, in Gas TIPS: Gas Research Institute, 5(2):4-11.

Nelson, E.A., and J.B. Gladden 2008. Full-scale treatment wetlands for metal removal from industrial wastewater. Environmental Geosciences, 15: 39-48.

Nissenbaum, A. 1975. The microbiology and biogeochemistry of the Dead Sea. Microb. Ecol. 2: 139-161.

Obire, O. and Nwaubeta, O. 2001. Biodegradation of refined petroleum hydrocarbons in soil. J. Appl. Sci. Environ. Mgt., 5, 43-46.

Odell, L.H., G.J. Kirmeyer, A. Wilczak, J.G. Jacangelo, J.P. Marcinko, and R.L. Wolfe, 1996. Controlling Nitrification in Chloraminated Systems. Jour. AWWA, 88(7): 86-98.

Oyanelel-Craver, V. A., Smith, J. A. 2006. Effect of quaternary ammonium cation loading and $\mathrm{pH}$ on heavy metal sorption to $\mathrm{Ca}$ bentonite and two organobentonites, Journal of Hazardous Materials, 137, 1102-1114.

Patterson, J. W., Allen, H. E., \& Scala, J. J. 1977. Carbonate precipitation for heavy metals pollutants. Water Pollution Control Federation, 49: 2397-2410.

Pierzynski, G.M., J.T. Sims, and G.F. Vance. 2005. Soils and Environmental Quality (3 ${ }^{\text {rd }}$ Edition), Taylor and Francis, Boca Raton, FL 569 p.

Postgate, J. R. 1984. The sulphate-reducing bacteria (second ed.). Cambridge: Cambridge University Press.

Prewitt, R.M. 1995. Power Plant Engineering: Site/Plant Arrangements. Springer. pp. 642658.

Rai, P.K., 2008. Heavy metal pollution in aquatic ecosystems and its phytoremediation using wetland plants: an ecosustainable approach. International Journal of Phytoremediation 10, 133-160.

Ray, J.P. and Engelhardt, F.R. 1992. Produced Water: Technological/environmental issues and solutions. New York: Plenum.

Reddy, K. R., \& DeLaune, R. D. 2008. Biochemistry of wetlands. Florida: CRC Press.

Rice, C.A., 1999, Waters co-produced with coalbed methane from the Ferron Sandstone in east-central Utah: Chemical and isotopic composition, volumes, and impacts of disposal: GSA Abstracts with Programs, 31: A385.

Rice, C.A., Ellis, M.S., and Bullock, J.H., Jr., 2000, Water co-produced with coalbed methane in the Powder River Basin, Wyoming: Preliminary compositional data: U.S. Geological Survey Open-File Report 00-372, p. 20.

Rice, C.A., Nuccio, V.F., 2000. Water produced with coalbed methane. U.S. Geological Survey Fact Sheet FS-156-00, p. 2.

Rickard, D. T. (1973). Limiting conditions for synsedimentary sulfide ore formation. Economic Geology, 68(5), 605-617. doi:10.2113/gsecongeo.68.5.605 
Rodgers, J.H. Jr. and Castle, J.W. 2008. Constructed wetland systems for efficient and effective treatment of contaminated waters for reuse. Environmental Geosciences, 15(1): $1-8$.

Rozkosny M. Salek J., and J. Salek. 2006. Water Balance of the Constructed Wetlands - A Study of the Macrophyte Evapotranspiration. Dias V., Vymazal J. (eds.) Proceedings of the $10^{\text {th }}$ International Conference on Wetland Systems for Water Pollution Control, MAOTDR and IWA, 23-29 September 2006. Lisbon, Portugal, pp. 123-130.

SAS Institute. 2002. Statistical Analysis System. Version 9. Cary, NC: SAS Institute.

Scott, G. and Crunkilton, R. 2000. Acute and Chronic Toxicity of Nitrate to Fathead Minnows (Pimephales promelas), Ceriodaphnia dubia, and Daphnia magna. Environmental Toxicology and Chemistry. SETAC, 19(12): 2918-2922.

Selinus, O., Alloway, B., Centeno, J. A., Finkelman, R. B., Fuge, R., Lindh, U., et al. (2005). Essentials of Medical Geology: Impacts of the Natural Environment on Public Health. London: Elsevier Academic Press.

Sell, N.J. 1992. Industrial Pollution Control: Issues and Techniques, Environmental Engineering Series, pp. 416.

Shamberger, R. J. (1983). Biochemistry of Selenium. New York: Plenum Press.

Shepherd, M.C., Shore, F.L., Mertens, S.K., \& Gibson J.S. 1992. Characterization of produced waters from natural gas production and storage operations: regulatory analysis of a complex matrix (In Ray J.P. \& Engelhardt F.R. (Eds.), Produced Water: Technological /Environmental Issues and Solutions (pp. 163-174) New York: Plenum Press.)

Siddique, T., Arocena, J. M., Thring, R. W., \& Zhang, Y. 2007. Bacterial reduction of selenium in coal mine tailings pond sediment. Journal of Environmental Quality, 36, 621-627.

Sinicrope, T. L., Langis, R., Gersberg, R. M., Busnardo, M. J. and Zedler, J. B. 1992. Metal removal by wetland mesocosms subjected to different hydroperiods, Ecol. Eng., 1: 309- 322.

Siron, R., Rontani, J.F., Giusti, G., 1987. Chemical characterization of a water soluble fraction (WSF) of crude oil. Toxicological and Environmental Chemistry. 15, 223229.

Snyder R.L. and C.E. Boyd. 1987. Evapotranspiration of Eichhornia crassipes (Mart.) and Typha latifolia L. Aquatic Botany 27: 217-227.

Sorrell, B.K., Tanner, C.C., and Sukias, J., 2002. Effects of water depth and substrate on growth and morphology of Eleocharis sphacelata: implications for culm support and internal gas transport. Aquatic Botany. 73, 93-106

Soucek, D.J., Cherry, D.S., and Zipper, C.E. 2001. Aluminum-dominanted acute toxicity to the cladoceran Ceriodaphnia dubia in neutral waters downstream of an acid mine drainage discharge. Canadian Journal of Fisheries and Aquatic Sciences, 58: 23962404.

Spacil, M. M. (2010). Constructed Wetland Treatment System for Risk Mitigation of Energy Derived Waters. Thesis. Clemson, South Carolina: Graduate School of Clemson University.

Stephenson, M.T. 1992. A Survey of Produced Water Studies. Produced Water: Technological/Environmental Issues and Solutions. J.P. Ray, F.R. Engelhardt eds. pp. 1-12. 
Sumi, Lisa. 2008. Shale Gas: Focus on the Marcellus Shale. For the Oil and Gas Accountability Project/Earthworks, May 2008.

Sundaravadivel, M. and Vigneswaran, S. 2001. Constructed wetlands for wastewater treatment. Crit. Rev. Environ. Sci. Technol., 31: 351-409.

Taniguchi, M., Fusaoka, Y., Nishikawa, T., and Kurihara, M. 2004. Boron removal in RO seawater desalination. Desalination 167: 419-426.

Tao, W., Hall, K.J., Duff, S.J., 2005. Performance evaluation and effects of hydraulic retention time and mass loading rate on treatment of woodwaste leachate in surface-flow constructed wetlands. Ecological Engineering 26, 252-265.

Tessier, A., Campbell, P. G. C., \& Bisson, M. (1979). Sequential extraction procedure for the speciation of particulate trace metals. Analytical Chemistry, 51(7), 847-851.

Thordsen, J.J., Kharaka, Y.K., Kakouros, E., and Ambats, G. 2007. The fate of inorganic and organic chemicals in produced water from the Osage-Skiatook Petroleum Environmental Research sites "A" and "B", Osage County, Oklahoma. US Geol. Surv. Open-File Report, OFR 2007-1055, 15 p.

Tomei, A. T., Barton, L. L., Lemanski, C. L., Zocco, T. G., Fink, N. H., Sillerud, L. O. 1995. Transformation of selenate and selenite to elemental selenium by Desulfovibrio desulfuricans. Journal of Industrial Microbiology, 14: 329-336.

Toussaint, M.W., Shedd, T.R., van der Schalie, W.H., and Leather, G.R. 1995. A comparison of standard acute toxicity tests with rapid-screening toxicity tests. Environ. Toxicol. Chem. 14: 907-915.

Trudinger, P. A., \& Swaine, D. J. (1979). Biogeochemical Cycling of Mineral-Forming Elements. New York: Elsevier/North-Holland Inc.

Tucker, M.E. 2001. Sedimentary Petrology: an introduction to the origin of sedimentary rocks. Wiley-Blackwell, pp. 262.

USEPA. 1991. Technical support document for water-quality-based toxics control. EPA 505/2-90-001. Washington, DC: United States Environmental Protection Agency.

USEPA, 1993. Memorandum: Office of Water Policy and Technical Guidance on Interpretation and Implementation of Aquatic Life Metals Criteria, Appendix A. United States Environmental Protection Agency, Washington, D.C.

USEPA. 1994a. Determination of Metals and Trace Elements in Water and Wastes by Inductively Coupled Plasma-Atomic Emission Spectrometry, Method 200.7. United States Environmental Protection Agency. Protection Agency.

USEPA, 1994b. Short-term methods for estimating the chronic toxicity of effluents and receiving water to freshwater organism. EPA/600/4-91/002. United States Environmental Protection Agency, Washington, D.C.

USEPA. 2000. EPA Office of Compliance Sector Notebook Project: Profile of the oil and gas extraction industry. EPA/310-R-99-006.

USEPA. 2002a. Hazardous Waste. EPA/40 CFR Part 148.

USEPA. 2002b. USEPA Office of Groundwater and Drinking Distribution System Issue Paper: Nitrification White Paper. Prepared by AWWA, 2002.

USEPA. 2002c.Short-Term Methods for Estimating the Chronic Toxicity of Effluents and Receiving Waters to Freshwater Organisms, $4^{\text {th }}$ edition. U. S. Environmental Protection Agency, Washington, D.C. EPA 821-R-02-013. 
USEPA, 2002d. Methods for the determination of total organic carbon (TOC) in soils and sediments. United States Environmental Protection Agency, Washington, D.C. NCEA-C-1282.

USEPA. 2004. Guidelines for Water Reuse. EPA/625/R-04/108.

USEPA. 2005. National Recommended Water Quality Criteria.

USEPA. 2006. National recommended water quality criteria. Office of Water, Office of Science and Technology, United States Environmental Protection Agency. http://www.epa.gov/waterscience/criteria/wqctable/nrwqc-2006.pdf.

USEPA. 2009. ECOTOXicology database system, version 4.0. United States Environmental Protection Agency. http://cfpub.epa.gov/ecotox/ecotox_home.cfm.

USGS, 2002. Produced Water database. United States Geological Survey.

Utvik, T.I.R. 1999. Chemical characterisation of produced water from four offshore oil production platforms in the North Sea. Chemosphere, 39(15): 2593-2606.

Veil, J.A., Puder, M.G., Elcock, D., and Redweik, R.J. 2004. "A White Paper Describing Produced Water from Production of Crude Oil, Natural Gas, and Coal Bed Methane," ANL Report under DOE (NETL) Contract W-31-109-Eng-38.

VWS. 2009. VWS Westgarth: The Water Treatment Expert in Upstream Oil \& Gas. Veolia Water Solutions \& Technologies.

Vymazal, J., Brix, H., Cooper, P., Raimund, R., Perfler, R., Laber, J. 1998. 'Removal mechanisms and types of constructed wetlands', in J. Vymazal, H. Brix, P. F. Cooper, M.B. Green and R. Hubert (eds.), Constructed Wetlands for Wastewater Treatment in Europe, Backhuys Publishers, Leiden, The Netherlands.

Ward, D.M., Brock, T.D., 1978.Anaerobic metabolism of hexadecane in sediments. Geomicrob. Journal. 1, 1-9.

Warner, J.B. 1971. Normal fluoride content of seawater. Deep Sea Res., 18: 1255-1263.

Watson, S.W., F.W. Valos, and J.B. Waterbury, 1981. The Family Nitrobacteraceae. In The Prokaryotes. Edited by M.P. Starr et al. Berlin: Springer-Verlag.

Western Governors' Association (WGA). 2006. Coal Bed Methane Best Management Practices Handbook-Appendix E. Washington, DC.

Wetzel, R.G. 2001. Limnology: Lakes and River Ecosystems, $3^{\text {rd }}$ Edition. Elsevier Science \& Technology Books. pp. 1006.

Wood, E.D., Armstrong, F.A.J., and Richards, F.A. 1967. Determination of Nitrate in Sea Water by Cadmium-Copper Reduction to Nitrite. J. Mar. Biol. Ass. U.K., 47: 2331.

Wyoming Department of Environmental Quality (WDEQ), 2001, SAP: Water Quality Rules and Regulations: Chapter 1. Department of Environmental Quality and Water Quality Division. Cheyenne, WY.

Zawislanski, P.T., H.S. Mountford, E.J. Gabet, A.E. McGrath, and H.C. Wong. 2001a. Selenium distribution and fluxes in intertidal wetlands, San Francisco Bay, California. J. Environ. Qual. 30: 1080-1091.

Zawislanski, P. T., Chau, S., Mountford, H., Wong, H. C., \& Sears, T. C. 2001 b. Accumulation of selenium and trace metals on plant litter in a tidal marsh. Estuarine, Coastal and Shelf Science, 52: 589-603.

Zhang, Y. \& Frankenberger, Jr., W. T. 2005. Removal of selenium from river water by a microbial community enhanced with Enterobacter taylorae in organic carbon coated sand columns. Science of the Total Environment, 346: 280-285. 
Zhang, Y., Okeke, B. C., \& Frankenberger Jr., W. T. 2008. Bacterial reduction of selenate to elemental selenium utilizing molasses as a carbon source. Bioresource Technology, 99: 1267-1273.

Zhou, N., Luo, H.-D., Li, N., Jia, Y.-z., Li, \& Y.-Q. 2009. Highly sensitive and selective spectrofluorimetric approach for the rapid determination of trace benzo[ $\alpha$ ]pyrene in drinking water and in solutions leached from disposable paper cups. Luminescence, doi: 10.1002/bio.1183.

Zobell, C. E. 1964. The occurrence, effects, and fate of oil polluting the sea. Advanced Water Pollution Research. 3, 85-118. 